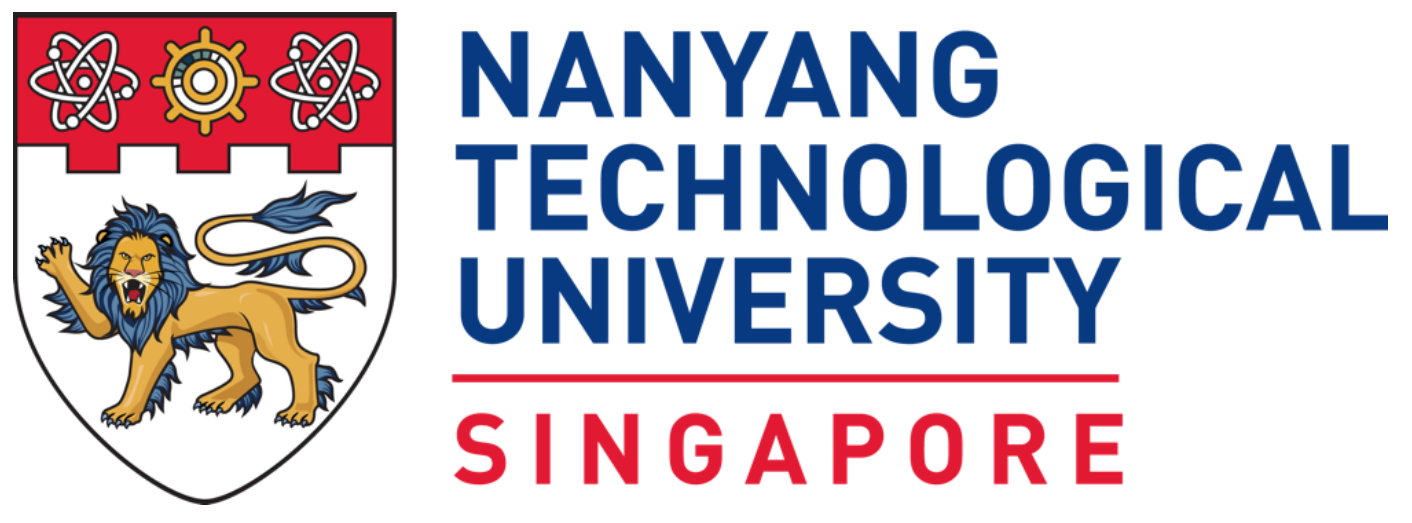

\author{
STUDIES ON DIGITAL \\ HOLOGRAPHY FOR ASPHERIC \\ SURFACE MEASUREMENT
}

\author{
WANG ZHAOMIN \\ SCHOOL OF MECHANICAL AND \\ AEROSPACE ENGINEERING \\ 2016
}




\section{STUDIES ON DIGITAL HOLOGRAPHY FOR ASPHERIC SURFACE MEASUREMENT}

\section{WANG ZHAOMIN}

School of Mechanical and Aerospace Engineering

A thesis submitted to the Nanyang Technological University in fulfillment of the requirement for the degree of Doctor of Philosophy

2016 


\section{ABSTRACT}

Digital holography is fast becoming a generalized technique for 3D precision measurement. By numerical reconstruction and propagation of a digital hologram, the optical field from an object and thus the amplitude and phase can be quantitatively reconstructed. The quantitative phase allows determination of the surface topography of the object with nanometer sensitivity.

Aspheric lenses are being increasingly used in optical system due to savings in costs and components. However, an aspheric is a complex surface designed using non-linear equations and hence fabrication is as best as can be measured. Various approaches

primarily using null interferometry have been proposed and used with limited success and slow speeds.

In this thesis, measurement and characterization of the aspheric lens with digital holography is studied. An adaptive optical element is introduced in the digital holography setup to measure aspheric lenses with different Numerical Aperture (NA). To measure the high gradient surface on the aspheric lens, a Dual-Wavelength Digital Holography (DWDH) with four phase unwrapping methods is proposed and demonstrated. For high resolution and large Field Of View (FOV), a novel synthetic aperture digital holography with first order spectrum based registration is suggested and analyzed. Finally, to characterize aspheric lenses, a linear simplified expression is applied to aspheric surface fitting, and a modified Zernike polynomials fitting method is proposed to evaluate the wavefront of the aspheric lens. 
Interferometric methods for aspheric lens testing needs a Null optic which provides the same wavefront as the lens under test. Thus a new Null optics has to be designed and fabricated for every aspheric lens to be tested. This will not only increase the measurement cost but additionally the fabrication process of the Null compensator is quite time-consuming and complex. Herein, a novel adaptive optical component Electrically Tunable Lens (ETL) is introduced to act as the compensator. The ETL can change its focal length which corresponds to different NA to match different aspheric lens. This provides extra flexibility in the measurement. Moreover, through doubleexposure approach, absolute phase is available which is very important for quantitative characterization.

Due to high gradients on the aspheric surface, single wavelength digital holography often fails to resolve the correct profile due to the limitation of axial resolution. DWDH is proposed to overcome this issue. Three existing phase unwrapping methods are discussed and modified for implementation. Moreover, a linear programming phase unwrapping method is proposed. Simulation is done to test these four methods under different levels of noise. At last, step structure and aspheric lens are tested to verify DWDH experimentally.

Synthetic aperture digital holography is next proposed to achieve large FOV. The first order spectrum based registration is proposed to do numerical reconstruction simultaneously during scanning. The experiment shows the proposed registration method is simple and straightforward. 
In the last part, a linear equation of the aspheric surface is derived and converted to orthogonal polynomials by Gram-Schmidt orthogonalization in Cartesian coordinate system. The aspheric parameters are then obtained by least squares fitting. The wavefront evaluation is performed by a modified Zernike polynomials fitting method. The method converts Zernike polynomials from Polar coordinate system into Cartesian coordinate system and orthogonalizes them. The mean value the wavefront is estimated in advance and removed to accelerate convergence. 


\section{ACKNOWLEDGMENTS}

I would like to express my sincere appreciation to my supervisor Prof. Anand Asundi for his patient supervising in digital holography, optical design, and paperwork. Thanks to his trust and support, I set up my network of researchers, engineers, and industry companies during my Ph.D. study.

I would like to thank Prof. Tian Ailing for her help in last ten years. During my MS study, she supervised me in precision measurement for one year. During my Ph.D. study, she helped me in research work and supported in attending the academic conferences.

I would like to thank Ms. Chee Oichoo for her help in my work in Singapore. Under her MOE-funded project, I learned about digital holography for the first time in my life.

I would like to thank Dr. Qu Weijuan for her help and support during my Ph.D. study. During last four years, we have cooperated on three MOE-funded projects. In these projects, I have learned a lot in mechanical design, optical design, and algorithm and software development.

I would like to thank Dr. Lim Han Leong for his help and support during last three years. Through our joint MOE-funded project, I really benefitted not only professional knowledge but also a lot of precious work experience. With his encouragement, I submitted a proposal for MOE funding (awarded) which greatly extends my work scope.

I would like to express my gratitude to my parents for their love. During past four years, I could not provide any financial support to them due to my heavy payload. Even visiting them was few and far between. 
I would like to express my love to my wife and daughter. During past four years, I could not spend too much time with them because of day-time work and night-time research. I even can count the number of weekends that I spent with them. Seeing my daughter's height grow from $82 \mathrm{~cm}$ to $112 \mathrm{~cm}$, during the course of my thesis, I would like to thank again to my wife. Without her support and giving, I could not insist on my Ph.D. work until present.

Finally, I would like to acknowledge the financial support from Ngee Ann Polytechnic, Optics and Photonics Society of Singapore and Peking University during my Ph.D. study for international conferences and communications. 


\section{TABLE OF CONTENTS}

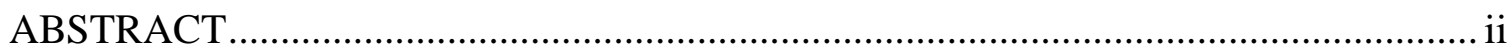

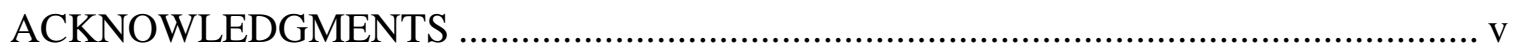

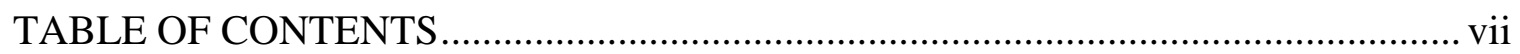

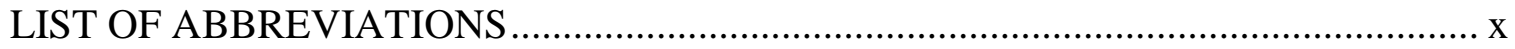

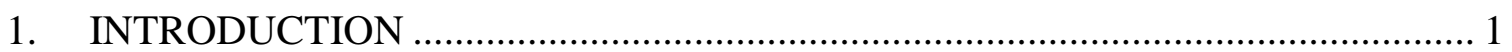

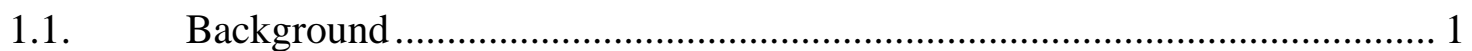

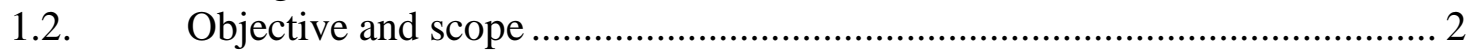

1.2.1. Tunable digital holography for measuring aspheric lens ............................... 2

1.2.2. Dual-wavelength digital holography for measuring steep gradient

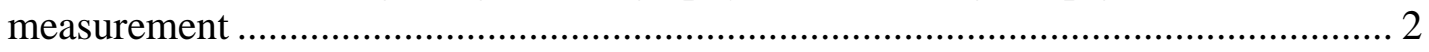

1.2.3. Synthetic aperture digital holography for large field of view measurement . 3

1.2.4. Characterization of the aspheric lens .......................................................... 3

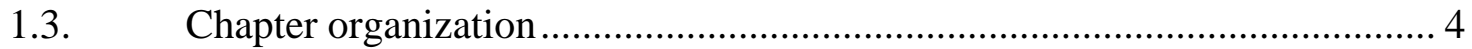

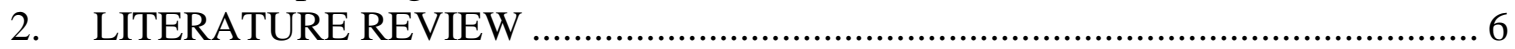

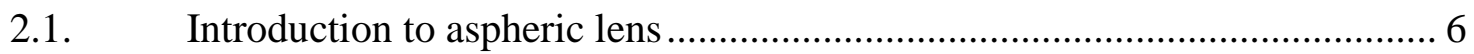

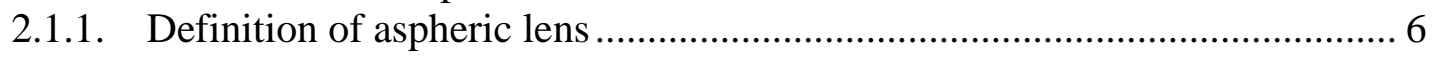

2.1.2. Applications of aspheric lens .................................................................. 7

2.2. Existing techniques for aspheric lens measurement .................................... 8

2.2.1. Interferometry with Computer Generated Hologram (CGH) for aspheric lens

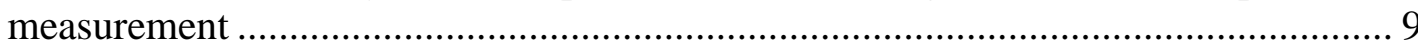

2.2.2. Shack-Hartmann wavefront sensor for aspheric surface form measurement 10

2.2.3. Stylus instrument for aspheric surface measurement................................... 11

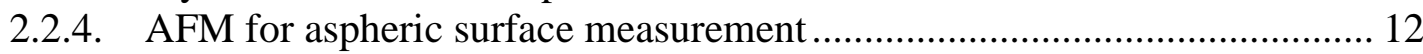

2.2.5. Zygo VeriFire Asphere for aspheric surface measurement .......................... 13

2.2.6. Interferometry with Point Source Array (PSA) for aspheric lens

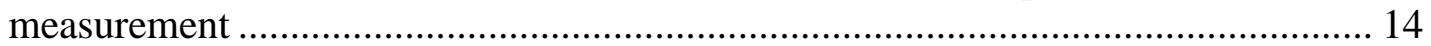

2.2.7. Deflectometry for aspheric surface measurement..................................... 15

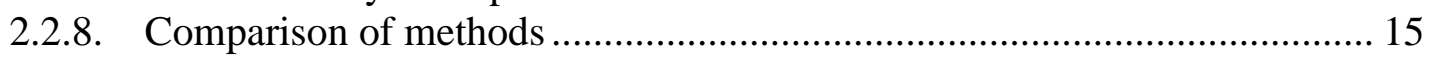

2.3. Introduction to digital holography ………........................................... 18

2.3.1. History of digital holography development ................................................ 18

2.3.2. Applications of digital holography ......................................................... 20

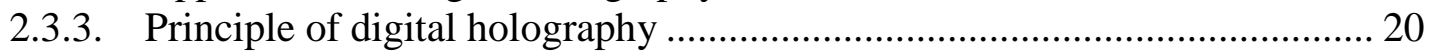

a) Fresnel transform method .................................................................... 23

b) Huygens convolution method .................................................................. 23

c) Angular spectrum method ...................................................................... 24

2.3.4. Flexible configurations in digital holography ........................................... 25

2.4. Chapter summary …………………………....................................... 25

3. DIGITAL HOLOGRAPHY WITH ELECTRICALLY TUNABLE LENS TO

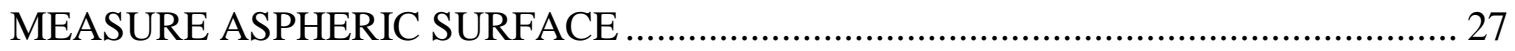

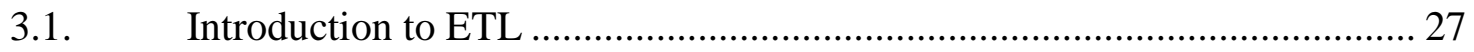

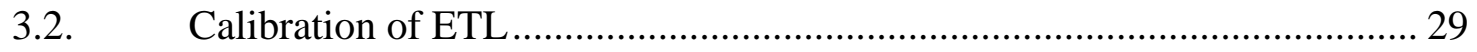

3.2.1. Digital holographic microscope for measurement of ETL .......................... 29 
3.2.2. Radius calculation by least squares method................................................. 33

3.2.3. Focal length calculation by Zemax .......................................................... 34

3.2.4. Mathematic modeling for tunable lens and its combination with offset lens 36

3.3. Principle of digital holography with ETL to measure aspheric surface....... 39

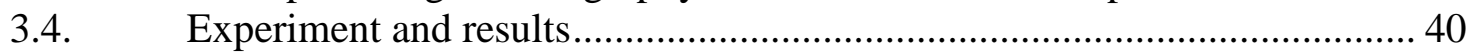

3.4.1. 4.5mm diameter aspheric lens testing ......................................................... 40

3.4.2. 25mm diameter aspheric lens testing ........................................................ 44

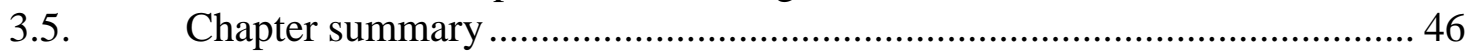

4. DUAL-WAVELENGTHS DIGITAL HOLOGRAPHY TO MEASURE ASPHERIC

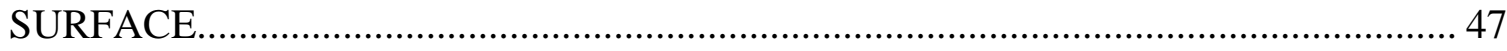

4.1. Dual-wavelength digital holography ........................................................ 47

4.2. Modification of existing phase unwrapping algorithms .............................. 49

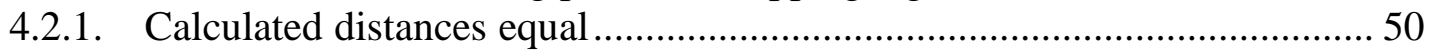

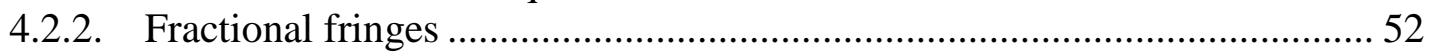

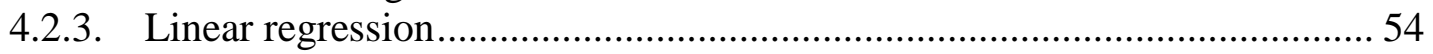

4.3. Principle of linear programing phase unwrapping algorithm ..................... 56

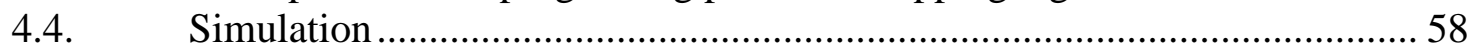

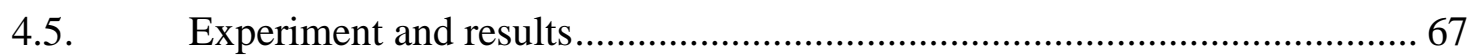

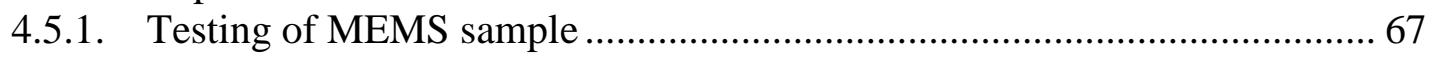

4.5.2. Testing of cylindrical lens........................................................................ 71

4.5.3. Testing of aspheric lens ....................................................................... 74

4.6. Chapter summary ………………………………………………........ 77

5. SYNTHETIC APERTURE DIGITAL HOLOGRAPHY TO MEASURE

ASPHERIC SURFACE ………………............................................................... 79

5.1. Lateral resolution improvement of synthetic aperture digital holography... 79

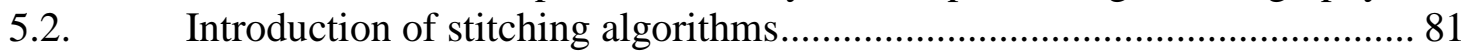

5.3. Principle of stitching algorithm based on first order spectrum.................... 84

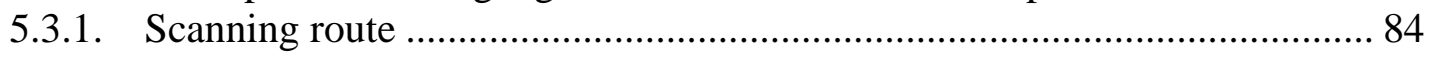

5.3.2. Fourier transform and first order spectrum filtering .................................. 85

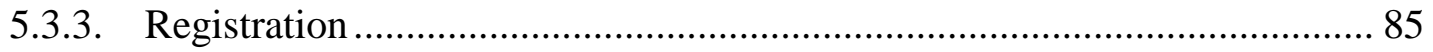

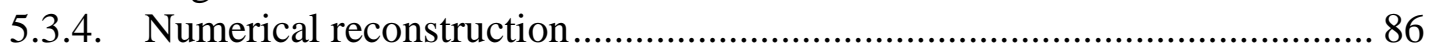

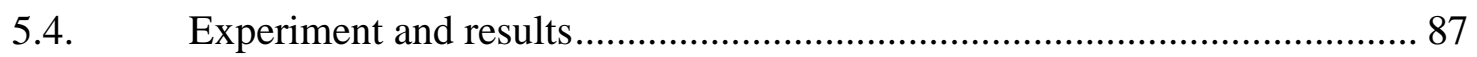

5.5. Chapter summary ................................................................................. 97

6. ASPHERIC LENS CHARATERIZATION …………..................................... 98

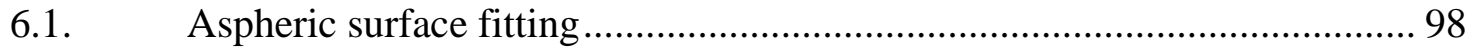

6.1.1. Conversion of aspheric surface expression .............................................. 100

6.1.2. Gram-Schmitt orthogonalization ........................................................... 102

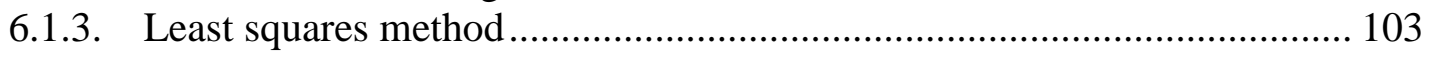

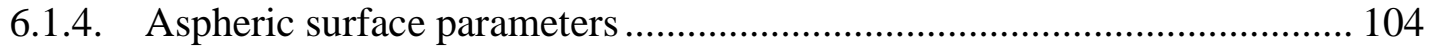

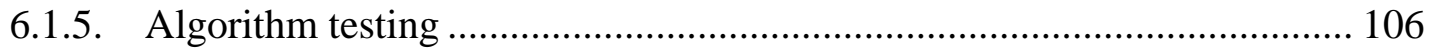

6.1.6. Experimental testing ............................................................................. 108

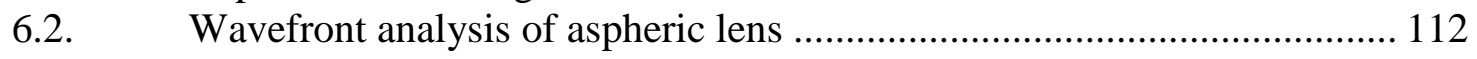

6.2.1. Wavefront represented by Zernike polynomials ....................................... 112

6.2.2. Aperture apodization............................................................................. 114

6.2.3. Generation orthogonal polynomials by Gram-Schmidt orthogonalization 117 
6.2.4. Least squares method ..................................................................... 119

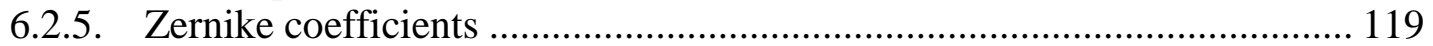

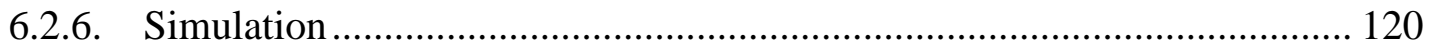

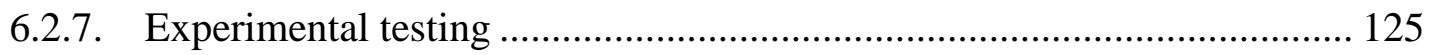

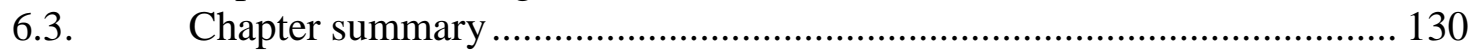

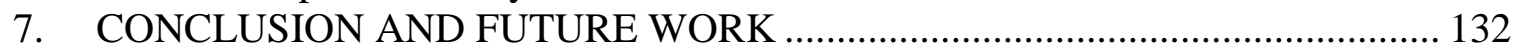

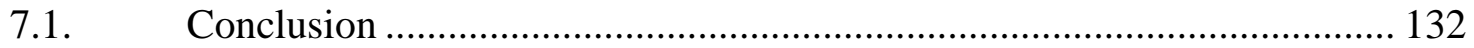

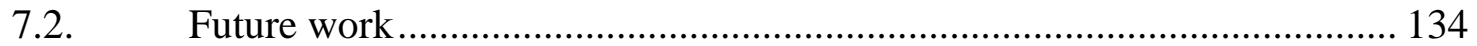

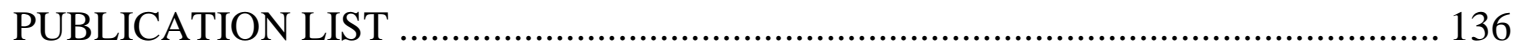

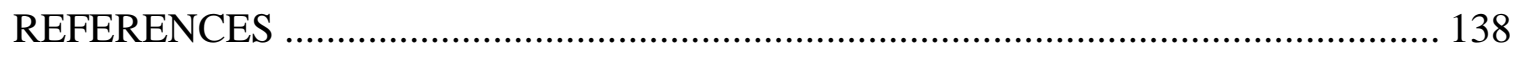




\section{LIST OF ABBREVIATIONS}

\begin{tabular}{|c|c|}
\hline AFM & Atomic Force Microscope \\
\hline $\mathrm{BS}$ & Beam Splitter \\
\hline $\mathrm{CCD}$ & Charge Coupled Device \\
\hline $\mathrm{CGH}$ & Computer Generated Hologram \\
\hline HDI & Heterodyne Displacement Interferometry \\
\hline DWDH & Dual-Wavelength Digital Holography \\
\hline ETL & Electrically Tunable Lens \\
\hline FFT & Fast Fourier Transform \\
\hline $\mathrm{FL}$ & Field Lens \\
\hline FOV & Field Of View \\
\hline L-BFGS & Limited memory-Broyden-Fletcher-Goldfarb-Shannon \\
\hline $\mathrm{LCD}$ & Liquid-Crystal Display \\
\hline LM & Levenberg-Marquartdt \\
\hline MA & Microlens Array \\
\hline MEMS & Micro-Electro-Mechanical Systems \\
\hline $\mathrm{MO}$ & Micro Objective \\
\hline NA & Numerical Aperture \\
\hline $\mathrm{PA}$ & Pinhole Array \\
\hline PSA & Point Source Array \\
\hline PSD & Position-Sensitive Detector \\
\hline PSI & Phase-Shifting Interferometry \\
\hline
\end{tabular}




\section{INTRODUCTION}

\subsection{Background}

The performance of high precision optical systems using spherical lenses is limited by the spherical shape of the lens resulting in aberrations. By using an aspheric lens, these aberrations can be reduced or even eliminated while at the same time reducing the need for corrective lenses thereby reducing the weight and the size of the system [1]. However, unlike spherical surface, the aspheric surface needs more complex design equation as well as expensive manufacturing process and cumbersome metrology. Due to the complexity of the aspheric surface, conventional metrological methods for spherical surface cannot be directly applied to measure aspheres. Therefore, suitable metrology tools with high accuracy, universal applicability, non-contact, large measurement volume and short measurement time are demanded in manufacturing industry and application of aspheric surfaces. Besides interferometric methods, digital holography seems to be a promising tool to meet the above requirements.

Digital holography is a quantitative phase imaging technique of acquiring and processing holographic measurement data (known as digital hologram), typically via a Charge Coupled Device (CCD) camera. The numerical reconstruction from the recorded digital hologram reproduces the optical field of an object in reasonable propagation distance. Therefore, digital holography is able to access the amplitude as well as the phase of the optical field. In particular, with the phase information, three-dimensional (3D) surface or optical thickness can be readily retrieved. Advantages such as nanoscale axial resolution, real-time measurement \& process, and low cost have made digital holography a 
competitive technique in optical precision measurement. It has been successfully applied in surface measurement, vibration testing, bioscience and many other fields [2-4].

\subsection{Objective and scope}

The objective of this study is to measure aspheric surface with digital holography and propose and develop new methodology for characterization the wavefront.

The scope of the work is

\subsubsection{Tunable digital holography for measuring aspheric lens}

A novel method for testing aspheric lens using digital holography with an ETL is proposed and experimentally verified. The ETL generates a tunable deformed wavefront which helps to decrease the high gradient on the surface of the aspheric lens. By decomposing the aspheric surface into two resolvable ones, its absolute phase can be determined using a double-exposure measurement. In this method, the wavefront generated by the ETL need not be identical to the aspheric surface as in conventional null interference system, but just sufficient to resolve the high gradient surface. On the other hand, the tunability of the ETL allows generation of variable wavefronts which can be used to test different aspheric lens. Furthermore, advantages of the ETL such as low cost, fast response, and compact configuration make the proposed method a promising technique for aspheric surface measurement.

\subsubsection{Dual-wavelength digital holography for measuring steep gradient measurement}

DWDH is analyzed to overcome the limitation in single wavelength digital holography. Three dual-wavelength phase unwrapping methods are discussed and modified for better 
implementation. Moreover, a linear programming phase unwrapping method is proposed. Simulation regarding four methods has been done by unwrapping step structures under different levels of Gaussian noise. As a result, three modified methods and our proposed method draw similar results with respect to simulated values. In the experiment, a MEMS sample, a cylindrical lens and an aspheric lens are measured to verify our method.

\subsubsection{Synthetic aperture digital holography for large field of view measurement}

By numerical reconstruction of the recorded digital hologram, the amplitude and phase information can be recovered with high resolution. However, the FOV in digital holography is limited by the physical size of the imaging sensor as well as the system magnification. Hence for small aperture aspheric lens, single shot measurement works well. However, for a larger aperture aspheric lens, single shot measurement is not adequate to cover the whole FOV. To overcome this problem, synthetic aperture digital holography is usually required. A first order spectrum based image registration is proposed and verified experimentally. As a result, it is simple and straightforward.

\subsubsection{Characterization of the aspheric lens}

Firstly, the aspheric parameters are calculated by a linear fitting method. A linear equation of the aspheric surface is derived and converted to orthogonal polynomials by Gram-Schmidt orthogonalization in Cartesian coordinate system. The aspheric parameters are then fitted by least squares method. Secondly, the wavefront evaluation is performed by a modified Zernike polynomials fitting method. The method converts Zernike polynomials from Polar coordinate system into Cartesian coordinate system and orthogonalizes them. The mean value the wavefront is estimated and removed to 
accelerate the convergence speed. Moreover, the weighted polynomials are applied in first four terms to accelerate the convergence speed.

\subsection{Chapter organization}

In chapter 2, different aspheric lens measurement techniques are investigated and reviewed. The concise principles and characteristics of these techniques are introduced. Their advantages and disadvantages are concluded. The principle of digital holography is introduced and compared with other techniques.

In chapter 3, digital holography with ETL to measure aspheric lens is presented. Nonlinearity of the ETL is demonstrated. The calibration of the ETL with digital holography is presented. The principle of measurement of the aspheric lens in digital holography is presented. Different aspheric lenses are tested in the experiment.

In chapter 4, DWDH is presented to measure aspheric lens. DWDH is theoretically analysed in improving the axial resolution of the system. Three dual-wavelength phase unwrapping method are investigated. Linear programming phase unwrapping method is proposed. Simulation is done to test the performance of four methods.

In chapter 5, synthetic aperture digital holography for measuring aspheric lens is investigated. A new registration method based on first order spectrum is presented. The method depends on the resolution of motorized stages to pre-calculate the composite surface size. Digital hologram in each scanning step is transformed on the final composite surface by Fast Fourier Transform (FFT). The first order spectrum is filtered and summed 
for numerical reconstruction. This method requires only one-time numerical reconstruction and removes the discontinuities by phase unwrapping.

In chapter 6, characterization of aspheric lens is presented. A simplified linear aspheric lens expression is derived. A fitting method for aspheric surface parameters based on orthogonal polynomials constructed by the simplified expression is presented. Wavefront fitting and analysing with Zernike polynomials are also presented.

In chapter 7, contributions of this thesis are concluded. Advantages and disadvantages of presented work are summarized. Future work is also discussed. 


\section{LITERATURE REVIEW}

\subsection{Introduction to aspheric lens}

Aspheric lens is an optical lens whose surface profile is not portions of a sphere or cylinder. Its complex surface can significantly reduce or eliminate spherical aberration, and at the same time suppress other high order optical aberrations compared to a spherical lens. Aspheric lens can be produced in several configurations: as one aspheric surface on a substrate, as a combination of aspheric surfaces with spherical surfaces or as a combination of several aspheric surfaces.

\subsubsection{Definition of aspheric lens}

According to ISO 10110-Part 12, an aspheric surface can be described by continuous mathematical functions

$$
z=f(r)=\frac{\frac{r^{2}}{R}}{1+\sqrt{1-(1+\kappa)\left(\frac{r}{R}\right)^{2}}}+\sum_{i=2}^{n} A_{2 i} r^{2 i}
$$

where $r$ is the lateral coordinate, $z$ is the sag and $R$ is the paraxial surface radius. $A_{2 \mathrm{i}}$ are the coefficients of the even order terms. The conic constant $\kappa$ is 0 for sphere, -1 for parabola, $<-1$ for hyperbola, between -1 and 0 for prolate spheroid and $>0$ for oblate spheroid.

This is visualized in Fig. 2.1. 


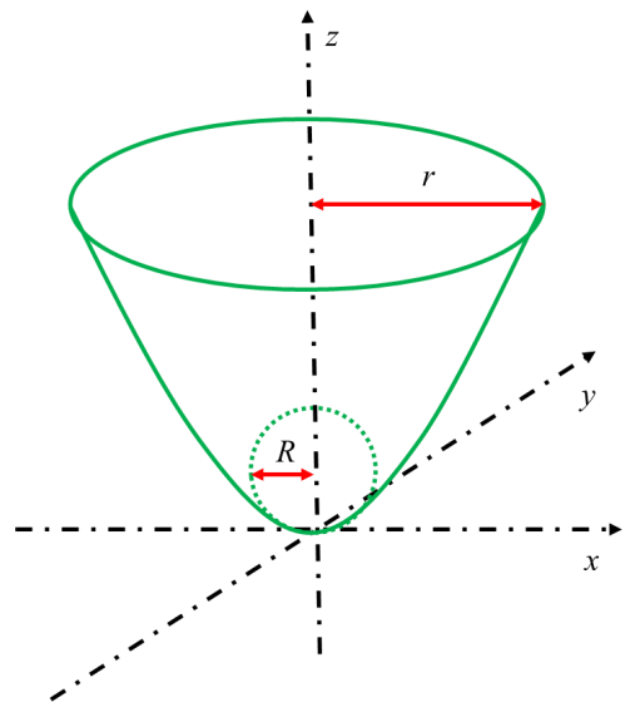

Figure 2.1 Schematic of an aspheric surface.

In addition, aspheric surface can be rotationally symmetric, axially symmetric, or completely asymmetric (free-form surfaces).

Dependent on the production volume, on the degree of asphericity, and on the required tolerance values, aspheric lenses can be manufactured by a variety of production methods, for example, by casting and injection molding of plastics, by blank pressing of glass, or by precise diamond turning.

\subsubsection{Applications of aspheric lens}

Aspheric lenses are used in optical systems to increase imaging quality, to reduce construction size or the number of elements, to save weight, to simplify the assembly process, or to reduce the overall manufacturing costs. Table 2.1 shows applications and advantages of aspheric lenses with respect to spherical lenses [1].

Table 2.1 Applications, advantages and status of aspheric lenses. 


\begin{tabular}{|c|c|c|}
\hline $\begin{array}{l}\text { Large quantities } \\
\text { illuminations }\end{array}$ & Improvement of imaging quality; reduction of cost & Production \\
\hline Laser collimator & Better imaging quality; reduction of cost; beam stability & Production \\
\hline Photo-optics & $\begin{array}{l}\text { Necessary for zoom systems; reduction of cost; better } \\
\text { imaging quality; smaller size }\end{array}$ & Production \\
\hline Large format film lenses & $\begin{array}{l}\text { Necessary for zoom systems; reduction of cost; better } \\
\text { imaging quality; smaller size }\end{array}$ & Production \\
\hline $\begin{array}{l}\text { Small quantities UV- } \\
\text { lithography }\end{array}$ & $\begin{array}{l}\text { better imaging quality; higher transmission at UV } \\
\text { wavelengths }\end{array}$ & production \\
\hline Aerial survey & Less weight; compact; radiation resistance; & In preparation \\
\hline $\begin{array}{l}\text { Space communication } \\
\text { correction plate for mirror } \\
\text { telescope }\end{array}$ & $\begin{array}{l}\text { Less weight; compact; radiation resistance; better imaging } \\
\text { quality }\end{array}$ & $\begin{array}{l}\text { Production in } \\
\text { study }\end{array}$ \\
\hline
\end{tabular}

\subsection{Existing techniques for aspheric lens measurement}

An important requirement for individual aspheric surface measurement is surface quality, for example, surface form, waviness, and roughness. In order to measure these parameters, different techniques are known to be used. Interferometry is the best known and most precise optical measuring technique for surface form, waviness and roughness measurement. Because of contacting measurement, a stylus instrument is regarded as a high reliable technique for measuring individual surface forms, waviness and roughness. By sampling the wavefront aberrations with the help of a microlens array, the ShackHartmann wavefront sensor is an economical choice for surface form measurement. Deflectometry is able to measure the surface form with quite simple projection configuration. Depends on the reaction of the forces between a mechanical probe and the sample, Atomic Force Microscope (AFM) is the most precise method to measure the roughness. 


\subsubsection{Interferometry with Computer Generated Hologram (CGH) for aspheric lens measurement}

When measuring a spherical surface, the illuminating "perfect" wavefront of the interferometer is focused into the center of the test surface's curvature. After auto reflection, the wavefront deformation, which propagates back to the interferometer, is twice the unknown surface deformation. By interference, a fringe pattern is generated; in another word, fringe deformations visualize the wavefront distortion. If the deformation is not too strong, the automated fringe analysis software is often able to determine the surface deformation.

However, in the case of aspheric surfaces, the illumination with a spherical wavefront may lead to phase deviations of the auto reflected wavefront, which are too large, leading to a fringe pattern with fringes that are too dense to be detected. The fringe pattern cannot, at all or partially, be retrieved by the analyzing software. In this case, a null corrector element is needed. Its purpose is to deform the incoming ideal wavefront from the interferometer in such a way that it corresponds to the ideal surface shape of the test surface. Then the auto reflected wavefront carries again only the actual deviation of the aspheric surface from its ideal function, the same as described by testing spherical surfaces. The difference to testing spherical surfaces is that the reference wavefront is no longer a perfect spherical wave but a complicated wavefront, which in turn is also very difficult to calibrate.

Due to the complexity of the null optics, CGH is often chosen as the null corrector [5, 6]. CGH can be generated by digitally computing a holographic interference pattern and printing it onto a mask or film for subsequent illumination by suitable coherent light 
source. Different structures for different additional functions, such as adjustment and calibration, can also be written on the same $\mathrm{CGH}$ to improve the measurement results. Some aspects need to pay attentions in application: before testing aspheric surface, the system needs to be carefully calibrated in case of the aberrations being transferred to aspheric surface; when the aspheric surface is non-symmetrical, the arrangement and adjustment are more difficult; adjustment errors of the surface under test and the CGH lead to similar fringe patterns, and the errors are therefore difficult to separate.

Typical parameters for interferometry with $\mathrm{CGH}$ are concluded in table 2.2.

Table 2.2 Typical operation parameters.

\begin{tabular}{ll}
\hline \multicolumn{1}{c}{ Parameters } & \multicolumn{1}{c}{ Typical range } \\
\hline Duration of measurement & $6-20$ days(include making Null corrector) \\
Measurement rate & $1 \mathrm{~min} /$ surface \\
Resolution in z & $\lambda / 4$ \\
Geometrical dimension of object measured & Convex: $2 \mathrm{~mm} \sim$ several meters \\
& Concave: limited by CGH diameter \\
\hline
\end{tabular}

\subsubsection{Shack-Hartmann wavefront sensor for aspheric surface form measurement}

In Shack-Hartmann sensor, a microlens array is placed in front of an imaging sensor. The distance between them is determined by placing the sensor chip exactly in the focal plane of the microlenses. Each microlens focuses its portion of the wavefront area onto a position-sensitive detector in an array. The average wavefront tilt across each microlens aperture results in a lateral shift of the perspective focal spot. An unaberrated wavefront produces a regular array of equidistant spots, whereas an aberrated wavefront produces a distorted spot pattern. Mapping the overall spot pattern distortions produces a map of the 
wavefront slopes, and an integration of these slopes will reconstruct the wavefront under test $[7,8]$.

Any aspheric surface can be imaged onto the microlens plate if an appropriate optical relay system is available [9-11]. Good zoom systems are recommended to achieve the flexibility required. The measured wavefront is a product of the unknown surface wavefront and the coherent transfer function of the relay optics. The latter must be known to retrieve the desired surface information. It should be noted that the Shack-Hartmann sensor is a slope-measuring device. From the slope data, the wavefront is obtained by numerical integration. For strong aspheric surfaces, a preshaping of the illumination wavefront by an element equivalent to a null lens is required.

This method is robust, compact, cost attractive, and its accuracy competes well with interferometry techniques. A drawback is the limited lateral resolution, which typically depends on the microlens number in the microlens array [12].

\subsubsection{Stylus instrument for aspheric surface measurement}

Stylus profilometry is an industry standard technique for measuring technical surface. It was originally used to measure the surface profile and to provide the amplified graph of the profile as well as simple roughness parameter such as Ra. Now they have been developed into a mature technology which can be used to measure $2 \mathrm{D}$ profile or $3 \mathrm{D}$ surface. Unlike surface-covering interferometry metrology, stylus profilemeter measuring does not need any null systems or compensators. Coordinates of sample points are referenced against an intrinsic coordinate system, which is supplied by the machine after a calibration process [13-15]. 
As a result, the stylus profilemeter can be applied in aspheric surface measurement. It has many advantages: the system does not need any Null optics; the system is never lured by the optical properties of a sample; the system has high vertical resolution; and the measurement result is highly reliable. However, it also has disadvantages: the stylus tip touches sample, and it may damage the optical surface; the stylus tip (often an artificial gemstone) radius smoothes the results and acts like a low-pass spatial filter; the tests needs scanning point by point to finish the whole surface measurement, and this process is time consuming $[16,17]$.

Table 2.3 lists typical operation parameters of stylus instrument.

Table 2.3 Typical operation parameters.

\begin{tabular}{ll}
\hline \multicolumn{1}{c}{ Parameters } & \multicolumn{1}{c}{ Typical range } \\
\hline Duration of measurement of $\varnothing 100 \mathrm{~mm}$ & $5 \mathrm{~min} \sim$ Several hours \\
Measurement rate & $0.01 \mathrm{~mm} / \mathrm{s} \sim 20 \mathrm{~mm} / \mathrm{s}$ \\
Resolution in z & $3 \mathrm{~nm} \sim 10 \mathrm{~nm}$ \\
Repeatability in z & $30 \mathrm{~nm} \sim 50 \mathrm{~nm}$ \\
Range in z & $0 \mathrm{~mm} \sim 50 \mathrm{~mm}$ \\
\hline
\end{tabular}

\subsubsection{AFM for aspheric surface measurement}

The AFM is a very high-resolution scanning probe microscopy, which axial resolution is sub-nanometer. It uses a mechanical probe to gather the information on the surface under testing. The precise scanning requires accurate and precise movements on electronic command. As result, this work is ensured and done by piezoelectric elements [18].

AFM is the most precise method to measure the roughness, where lateral dimensions $\leqslant$ $5 \mathrm{~nm}$ can be resolved. The depth resolution can be $\leqslant 0.05 \mathrm{~nm}$. One drawback is the small measurement field, typically $10 \mu \mathrm{m} \times 10 \mu \mathrm{m}$ or $100 \mu \mathrm{m} \times 100 \mu \mathrm{m}$. The fields can be 
extended by different techniques such as stitching; however, a high precision stage is needed. The AFM scanning speed is another big limitation. The relatively slow scanning rate often results in thermal drift in AFM imaging. Furthermore, AFM images are at the same time affected by other factors, such as hysteresis, nonlinearity, and cross-talk between the $x, y, z$ axes and creep of the piezoelectric material, which often require image processing for improvement.

\subsubsection{Zygo VeriFire Asphere for aspheric surface measurement}

The Zygo VeriFire is a new product for measuring aspheric lenses that employs both a mechanical Phase-Shifting Interferometry (PSI) combined with Heterodyne Displacement Interferometry (HDI) but in a different arrangement [19]. The system uses a transmissive sphere to generate spherical wavefronts, and then move the along its symmetrical axis. Thus, a stack of interferograms of the part can be captured in annular zones. The zone on the part and the position of the stage are corresponded based on where the optical surface and spherical wavefront will have a common tangent. Therefore a fringe pattern with a resolvable fringe density will be produced by the reflection. According to the distance of these zones' relative apex to the zone and scan position determined with the HDI, these zones are put together. Owing to the capabilities of the HDI, the apex radius and surface irregularity are measured at the same time and relative to each other.

There are some restrains on the aspheric lens: it needs to be symmetric axially; it must have a measurable apex; the defined aspheric equation is required to be known; the lens cannot have reversing curvature to be measured with the VeriFire Asphere instrument. 
Table 2.4 shows typical capability of the system.

Table 2.4 Typical operation parameters.

\begin{tabular}{ll}
\hline \multicolumn{1}{c}{ Parameters } & \multicolumn{1}{c}{ Typical range } \\
\hline Range in lateral & $1 \mathrm{~mm}-130 \mathrm{~mm}$ \\
Duration of measurement one circle & $2 \sim 8$ minutes \\
Resolution in $\mathrm{z}$ & $0.08 \mathrm{~nm}$ \\
Surface measurement repeatability & $\leq 5 \mathrm{~nm}(\lambda / 125)$ \\
Range in $\mathrm{z}$ & $0 \mathrm{~mm} \sim 850 \mathrm{~mm}$ \\
\hline
\end{tabular}

\subsubsection{Interferometry with Point Source Array (PSA) for aspheric lens measurement}

This approach makes use of a modified Twyman-Green interferometer $[20,21]$. By adopting multiple sources to illuminate different angles of the aspheric surface, the gradient is compensated, and as a result interference fringes are reduced. Different light sources are triggered in the measurement, in which the reference beam is always fixed. This method is able to measure strong aspheric departures while at the same time getting rid of high fringe densities and vignetting artifacts on the detector. It does not need any mechanical motion in testing and is capable of triggering multiple sources simultaneously.

Because the incident angle of the test wavefront to the test surface is no longer perpendicular, mismatch in optical paths may happen. To correct the errors, characterization is required. Because there is significantly difference between the optical path of the test beam and reference beam, it needs to make clear the sources of additional aberrations. Moreover, due to the high gradients on the tested surfaces, the imaging sensor may not be able to resolve the high density fringes. Thus, effects such as the wavefront vignetting must be taken into consideration. It should be noted that the optimization algorithm is able to correct the polynomial description of the aspheric 
surface. In this method, high frequency details of the surface are not modeled. Therefore, an extra step is required to compute the difference between the measured phase and the shape of the asphere reconstructed by optimization.

\subsubsection{Deflectometry for aspheric surface measurement}

Deflectometry is to observe a grating-like structure reflected off the test surface $[22,23]$.

The image of the grating-like structure will be deformed according to the test object shape. The surface form can be obtained by integration of the surface gradients, the slope angles. With PSI, this method can reconstruct the shape of measured object.

Deflectometry has advantages such as no need for complicated assistant optics, simple calibration, and anti-noise ability. However, drawbacks are also obvious in this method. The fringe patterns corresponding to the phase is the main source of the error. The system resolution is limited by the resolution of Liquid-Crystal Display (LCD). Moreover, the movement of the LCD and the CCD is required to be accurately controlled.

\subsubsection{Comparison of methods}

When $\mathrm{CGH}$ is required as null optics in testing aspheric surface, its design and manufacturing is a complicated and expensive process and limited to individual aspheric surfaces. The Shack-Hartmann wavefront sensor is suitable for aspheric surface form; but again, for the Shack-Hartmann wavefront sensor, a shape and pupil adaptation by an optical system is needed, and it is limited in lateral resolution. Stylus instrument can obtain aspheric surface form, waviness and roughness with high accuracy. However, it may damage the optical surface, and the measurement process is time-consuming. AFM has the best resolution in either vertical or lateral direction of measurement. Two obvious 
drawbacks of AFM exist: its detection area is very small; and its scanning is timeconsuming. Zygo VeriFire as a commercial product uses PSI and HDI in interferometer. It has nanoscale vertical resolution and large measurement range. However, this product is expensive and time-consuming. Interferometry with PSA uses structured illumination in measurement of aspheric surface. It can evaluate the aspheric surface by fitting the spherical surface in advance. But this method does not model high frequency component, it leads to final result containing high order system aberrations. The alignment also affects the final result, which makes this method not robust. Deflectometry can measure aspheric surface with very simple configuration, but its resolution is limited by the projector or the grating.

Digital holography is a quantitative interferometry which utilizes off-axis interferometer to generate and record interferogram, and then reconstruct the optical field by diffraction theory. In transmission geometry, the accuracy can be kept below 15nm. A Microscope Objective (MO) is often introduced between the object and the camera, as a result, the lateral resolution can be greatly improved. In the case of $4.65 \mu \mathrm{m}$ CCD pixel size and $40 \mathrm{X}$ MO, resolution can be kept commonly below $116 \mathrm{~nm}$.

Digital holography is real-time imaging technology. In comparison with other interferometry methods, digital holography only needs to record one image instead of multiple images or more and the reconstruction method is much simpler.

Digital holography system contains fewer optical elements and thus is less complex and easier to adjust and phase aberration due to optics is less severe. It also means that digital holography is a low cost technology. 
Digital holography is capable to provide more information than other interferometry methods in that not only the image plane but the whole complex optical field in the volume between CCD plane and image plane is calculated. Furthermore, by adjusting reconstruction distance, digital holography is capable of reconstructing the optical filed at different position along the optical axis. This capability enables digital holography in numerical autofocusing.

Digital holography is extendable for large range measurement with synthetic aperture technology. With synthetic aperture and stitching technologies, digital holography can greatly extend the measurement area.

To reduce the cost and to improve the efficiency of the production of aspheric surfaces, "in-process" metrology is desirable. Therefore, digital holography is chosen for advanced study of aspheric surface measurement. Further details of the synthetic aperture digital holography technique are introduced in the following chapter. Table 2.5 concludes and compares the characteristics of all the measurement methods discussed in this chapter.

Table 2.5 Comparison of aspheric surface measuring methods.

\begin{tabular}{|c|c|c|c|c|c|c|c|c|}
\hline Measure principle & $\begin{array}{c}\text { Axial } \\
\text { resolution }\end{array}$ & $\begin{array}{l}\text { Measure } \\
\text { volume }\end{array}$ & Cost & $\begin{array}{c}\text { Time } \\
\text { consuming }\end{array}$ & Damage & Form & $\begin{array}{l}\text { Waviness } \\
\qquad \& \\
\text { roughness }\end{array}$ & $\begin{array}{c}\text { Optical } \\
\text { performance } \\
\text { evaluation }\end{array}$ \\
\hline
\end{tabular}




\begin{tabular}{|c|c|c|c|c|c|c|c|c|}
\hline $\begin{array}{l}\text { CGH } \\
\text { interferometry }\end{array}$ & Nanoscale & Large & High & Yes & No & No & No & Yes \\
\hline Shack-Hartmann & Nanoscale & Large & Low & No & No & Yes & No & Yes \\
\hline Stylus instrument & Nanoscale & Large & High & Yes & Yes & Yes & Yes & No \\
\hline $\begin{array}{l}\text { PSI and MDI } \\
\text { interferometry }\end{array}$ & Nanoscale & Large & High & Yes & No & Yes & Yes & No \\
\hline AFM & Nanoscale & Very small & High & Yes & No & No & Yes & No \\
\hline $\begin{array}{l}\text { PSA } \\
\text { interferometry }\end{array}$ & Nanoscale & Small & Low & No & No & No & No & Yes \\
\hline Deflectometry & Microscale & Large & Low & No & No & Yes & No & No \\
\hline $\begin{array}{l}\text { Digital } \\
\text { holography }\end{array}$ & Nanoscale & Large & Low & No & No & Yes & Yes & No \\
\hline
\end{tabular}

\subsection{Introduction to digital holography}

\subsubsection{History of digital holography development}

Conventional holography was invented by Dennis Gabor in 1948 during his research to electronic microscopy. Holography is a novel method to record and reconstruct the phase and amplitude of a wave field [24, 25]. A wave field scattered from an object forms the object beam which then interferes with the reference beam and generates the inference pattern (hologram). The hologram is often recorded photographically on a flat plate of silver gelatin. The information of the whole three-dimensional wave field is contained in it. This information is coded in form of interference fringes. People usually cannot see these fringes because their high spatial frequencies. By illuminating the hologram with a duplicated reference wave, one can reconstruct the object wave again. A threedimensional image with all features of perspective and depth of focus can be observed. 
Due to the lack of high coherence and intensity light sources [26, 27], the holography was mostly applied to X-ray and electron microscopy in the 1950s [28]. Two critical inventions greatly accelerated the development of holography. The first one was the invention of laser which is true coherent light source. The other is that Emmett Leith and Juris Upatnieks [29-31] introduced the off-axis illumination in holography. Off-axis configuration is able to separate the conjugate and zero-order images which are overlapped in Gabor's on-axis configuration.

The principle of numerical reconstruction of a hologram was first demonstrated by Joseph Goodman et al in 1967 [32]. They optically sampled enlarged parts of in-line and Fourier holograms which were recorded on a photographic plate, and numerically reconstructed these digital holograms.

In 1994, CCD camera was introduced by Schnars and Jüptner to record holograms in offaxis Fresnel configurations, and they experimentally showed the capability of digital holography in metrology of macroscopic objects [33-35]. Till then, the imaging sensors and powerful computers had developed to a sufficient level to realize numerically acquisition and reconstruction of the digital hologram, and more and more researchers began to focus on related new techniques as well as applications. In 1999, quantitative digital holographic microscopy was demonstrated Christian Depeursinge's group, which directly reconstructs the tomography of a surface with less than a few nanometer effective noises $[36,37]$. In digital holography, the phase is reconstructed in real-time. In particular, the phase allows quantitative image processing which is infeasible in real space holography [38]. 


\subsubsection{Applications of digital holography}

Since middle nineties of the last century, number of techniques have been developed to improve the performance and to explore new applications. Different numerical reconstruction methods are developed to reconstruct object wave field [39]. Error compensation and noise suppression algorithms are proposed to improve the precision and accuracy of the digital holographic system [40-48]. Multiwavelength measurement is applied to get higher resolution in vertical direction [49-52]. Phase shifting technique is applied to digital holography to avoid frequency filtering [53-64]. Digital holography has been successfully applied in measuring MEMS and micro-optics [65-73]. It also has been used in bio measurement and monitoring [69, 74-88].

\subsubsection{Principle of digital holography}

In digital holography, a digital hologram is generated by interference of object wave $E_{o}$ and reference wave $E_{R}$. In the hologram plane, the recorded two-dimensional (2D) intensity distribution $I_{H}(x, y)$ can be expressed as follows

$$
\begin{aligned}
I_{H}(x, y) & =\left|E_{O}+E_{R}\right|^{2} \\
& =\left|E_{O}\right|^{2}+\left|E_{R}\right|^{2}+E_{O}^{*} E_{R}+E_{R}^{*} E_{O}
\end{aligned}
$$

Where $\left|E_{o}\right|^{2}$ and $\left|E_{R}\right|^{2}$ are intensities of the object and reference waves, respectively. $E_{o}^{*} E_{R}$ and $E_{R}^{*} E_{o}$ are two interference terms where $E_{o}^{*}$ and $E_{R}^{*}$ are the complex conjugates of the two waves. In conventional holography, the interference pattern is usually recorded on a transparent photographic plate to represent hologram. 
In order to reconstruct, another reference field $E_{R}^{\prime}$ is used to illuminate the hologram, so that optical field gives

$$
\begin{aligned}
E & =E_{R}^{\prime} I_{H}(x, y) \\
& =E_{R}^{\prime}\left(E_{O}+E_{R}\right)\left(E_{O}+E_{R}\right)^{*} \\
& =E_{R}^{\prime}\left|E_{O}\right|^{2}+E_{R}^{\prime}\left|E_{R}\right|^{2}+E_{R}^{\prime} E_{O}^{*} E_{R}+E_{R}^{\prime} E_{R}^{*} E_{O}
\end{aligned}
$$

The first two terms are related to the zero-order terms and represent the noninterfering intensity patterns of the object and reference waves separately. The last two terms in Eq. 2.3 are the interference terms and they produce two conjugate images of the object. The third term $E_{R}^{\prime} E_{o}^{*} E_{R}$ produces a real image located on the other side of the hologram. If $E_{R}^{\prime}=E_{R}^{*}$, the real image is produced by a replica of the conjugate of the object wave multiplied by the reference intensity $\left(\left|E_{R}\right|^{2} E_{o}^{*}\right)$. The fourth term $E_{R}^{\prime} E_{R}^{*} E_{o}$ produces a virtual image located at the position initially occupied by the object. If the reconstruction is done by illuminating the hologram with a replica of the reference wave $\left(E_{R}^{\prime}=E_{R}\right)$, the virtual image is produced by a replica of the object wave multiplied by the reference intensity $\left(\left|E_{R}\right|^{2} E_{o}\right)$

In conventional holography, the condition $E_{R}^{\prime}=E_{R}$ or $E_{R}^{\prime}=E_{R}^{*}$ is generally required. Especially for volume or thick holograms, Bragg condition for the illumination wave is defined in the thickness of the photographic emulsion for recording the interference. This Bragg condition also acts on the wavelength of the illuminating wave and if light with a broad spectrum (white light) is used for the reconstruction, only the "correct" wavelength will participate to the image formation. For thin holograms, 3D images can be observed 
even if the condition $E_{R}^{\prime}=E_{R}$ or $E_{R}^{\prime}=E_{R}^{*}$ is not strictly satisfied. However, it may reduce the quality of the reconstructed images, especially their resolution.

In Gabor's original work, the hologram was recorded in inline geometry. In which, the reference wave and the object wave follow parallel directions. In this case, the four components in the reconstructed wavefront propagate along the same direction and thus cannot be distinguished. In an off-axis configuration, $E_{o}$ and $E_{R}$ meet at the hologram plane with different directions. Because the different terms of the interference have different spatial frequencies, and as a result, these terms would propagate along different directions in reconstruction. Here, a reference wave is assumed as [36]

$$
E_{R}=\left|E_{R}\right| \exp \{i k x \sin \theta\}
$$

where $k=2 \pi / \lambda$ is the wave number and $\theta$ is the angle between the reference wave and the normal to the hologram. $I_{H}(x, y)$ becomes

$$
\begin{aligned}
I_{H}(x, y) & =\left|E_{O}+E_{R}\right|^{2} \\
& =\left|E_{O}\right|^{2}+\left|E_{R}\right|^{2}+E_{O}^{*}\left|E_{R}\right| \exp \{i k x \sin \theta\}+\left|E_{R}\right| \exp \{-i k x \sin \theta\} E_{O}
\end{aligned}
$$

The phase factor $\exp \{i k x \sin \theta\}$ appearing in the third term means that the wave which produces the real image propagates with an angle $\theta$. The phase factor $\exp \{-i k x \sin \theta\}$ in the fourth term indicates that the wave which produces the virtual image propagates with an angle $-\theta$ with respect to the direction of the illumination wave $E_{R}^{\prime}$. As a consequence, the conjugate images can be observed separately along distinct direction and the zero order $\left(E_{R}^{\prime}\left|E_{o}\right|^{2}+E_{R}^{\prime}\left|E_{R}\right|^{2}\right)$ propagates along the $E_{R}^{\prime}$ directions. 
When we get the intensity $I_{H}$, there are mainly three numerical reconstruction methods to recover the object wave $E_{o}(x, y, d)$ in digital holography. These methods need different times in Fourier transform due to different theories.

\section{a) Fresnel transform method}

This method makes an approximation of Fresnel diffraction into a Fourier transform which can correspondingly be implemented by a computer with high efficiency. The optical field propagates from a plane at $z=0$ to another plane $z=d$ along the $z$-axis can be written as [89]

$$
E(x, y, d)=2 \pi \exp \left[\frac{i \pi}{\lambda d}\left(x^{2}+y^{2}\right)\right] \mathcal{F}\left\{E_{R}^{*} I_{H}\left(-\frac{i}{\lambda d}\right) \exp \left(\frac{2 \pi d}{\lambda}\right) \exp \left[\frac{i 2 \pi}{\lambda d}\left(x^{2}+y^{2}\right)\right]\right\}\left[f_{x}, f_{y}\right]
$$

where $\mathcal{F}\{\}$ represents Fourier transform, and $(x, y)$ is coordinated in hologram plane. When the hologram is recorded on CCD with width $L_{x}$ and height $L_{y}$, the spatial frequency can be expressed as $f_{x}=1 / L_{x}$ and $f_{y}=L_{y}$.

The Fresnel Transform Method (FTM) only needs a single Fourier transform which can be readily implemented by a computer.

\section{b) Huygens convolution method}

The optical field can be computed by the Huygens convolution integral [89]

$$
E(x, y, d)=\mathcal{F}^{-}\left\{\mathcal{F}\left\{E_{R}^{*} I_{H}\right\}\left[f_{x}, f_{y}\right] \mathcal{F}\{P S F\}\left[f_{x}, f_{y}\right]\right\}[x, y]
$$

where $\mathcal{F}^{-}\{\}$represents inverse Fourier transform and the Huygens Point Spread Function (PSF) is

$$
P S F=-\frac{i}{\lambda d} \exp \left[i k \sqrt{x^{2}+y^{2}+d^{2}}\right]
$$


The Huygens Convolution Method (HCM) requires two Fourier transforms and one inverse Fourier transform. Unlike FTM, the pixel resolution in HCM does not depend on the propagation distance because of the combination of Fourier transform and its inverse transform. In the short distance reconstruction, HCM is better than FTM, and the minimum distance for HCM is similar to FTM. The reason for the improvement is mainly because of the use of spherical PSF instead of parabolic one.

\section{c) Angular spectrum method}

The optical field reconstructed by Angular Spectrum Method (ASM) gives [90]

$$
E(x, y, d)=\mathcal{F}^{-}\left\{\mathcal{F}\left\{E_{R}^{*} I_{H}\right\}\left[f_{x}, f_{y}\right] \exp \left[i \frac{2 \pi}{\lambda} d \sqrt{1-r^{2}}\right] \operatorname{circ}(r)\right\}[x, y]
$$

where $r$ is

$$
r=\sqrt{\left(\lambda f_{x}\right)^{2}+\left(\lambda f_{y}\right)^{2}}
$$

This method requires one Fourier transforms and one inverse Fourier transform. Angular spectrum method uses a cluster of plane wave with different spatial angles to simulate wavefront propagation. Thus the propagation process can be regarded as a linear, dispersive spatial filter with a finite bandwidth. In the frequency plane, the filter is zero outside a circular region with radius $r$. Within the circular bandwidth, the modulus of the transfer function is unity but frequency dependent phase shifts are introduced. The phase dispersion of the system is most significant at high spatial frequencies and vanishes as both $f_{x}$ and $f_{y}$ approach zero. In addition, for any fixed spatial frequency pair, the phase dispersion increases as the distance of propagation $d$ increases. 


\subsubsection{Flexible configurations in digital holography}

Michelson and the Mach-Zehnder are two main geometries adopted in digital holographic microscopy [3]. The Michelson interferometer is often used for reflective object measurement. By placing transparent specimen before a mirror, it also can be arranged for double-pass transmission. While the Mach-Zehnder interferometer is more suitable for transparent object measurement. It requires more components but also offers more flexibility in alignment.

In the meanwhile, the configurations may include other components, such as apertures, attenuators, polarization optics, piezo-mounted optics, acousto-optic, electro-optic modulators, liquid crystal phase modulator, and so on. Experimental configurations often apply horizontal arrangement because of convenient operation and adjustment, while vertical arrangement is more suitable for the sample which can be only placed horizontally.

\subsection{Chapter summary}

In summary, introduction with respect to aspheric lens is done first. In the introduction, the definition of the aspheric surface is described according to ISO standard. The aspheric lens consists of at least one aspheric surface. The applications of aspheric lenses are also concluded.

Different methods for measuring aspheric lens are viewed and summarized. Interferometry with $\mathrm{CGH}$ gives high-resolution inspection results, but also has to stand by the high cost and long-term fabrication period. Shack-Hartmann wavefront sensor can detect the form of an aspheric lens in real-time, but the result is in low resolution. The 
stylus instrument provides the most reliable results, but may damage the surface. AFM is undoubtedly the most high-resolution inspection method, but its detection area is too small and the detection process is time-consuming. Interferometry combined with PSI and DMI could achieve high axial resolution, but it needs much pre-knowledge about the aspheric lens and the instrument itself is quite expensive. Interferometry with PSA uses structured illumination to measure the aspheric lens. This method requires pre-calibration and complex reconstruction computation. Deflectometry requires very simple configuration and is able to achieve large FOV in measuring aspheric lens, but its resolution is limited by LCD and the accuracy depends on the mechanical motion.

Digital holography is quantitative phase imaging technique which can do numerical reconstruction in real-time. Moreover, this technique uses FTM to do reconstruction which greatly reduces the hardware cost with respect to interferometry with PSI. As a competitive technique, digital holography is a reasonable choice for the measurement of aspheric lens. 


\section{DIGITAL HOLOGRAPHY WITH ELECTRICALLY TUNABLE LENS TO MEASURE ASPHERIC SURFACE}

Digital holography as a well-established high precision 3D imaging technique is capable of numerically reconstructing the optical field of an object at any propagation distance $[2,3]$. It is capable of reconstructing the phase and amplitude of the object simultaneously. Along with fast reconstruction speed, high axial resolution, and low cost, it has become a powerful tool in quantitative phase imaging. However, testing of aspheric lenses is difficult due to high gradients on the aspheric surface resulting in high fringe densities which cannot be resolved and measured. In fact, digital holography also encounters the same problem when measuring an aspheric lens.

In this chapter, a new adaptive optical element, ETL [91, 92], is added to a digital holographic system to overcome the above problem. The electrical tuning power allows ETL to generate a suitable wavefront that decreases the high gradients of the aspheric surface. Before applying the ETL, its performance is firstly calibrated by digital holographic system. Then a Mach-Zehnder interferometer with the ETL is developed and demonstrated for the aspheric lens measurement. The experimental results are implemented and in good agreement with factory data for two sample aspheric lenses.

\subsection{Introduction to ETL}

ETL is a novel current controlled adaptive optical component which can sweep its tuning power in a specified range. Over traditional optics, it is compact in design, fast response, low power consuming, and less tolerance sensitivity. Since the advent of the technology, it has been successfully applied in many fields and shows great potential especially in quantitative tuning applications. A focus shifting unit using ETL instead of the control 
architecture and use of moving components is carried out in 3D laser material processing [93]. With a diffuser mounted on an elastic membrane and moved in a circle using electroactive polymers, planar circular oscillation of the diffuser can be generated to reduction laser speckle noise $[94,95]$. By performing an axial focus scan using ETL, depth extended OCT is realized without trade-off high lateral resolution [96]. Volume imaging is achieved with fast 3D scanning [97]. Different layers of interested are rapidly focused [98]. In quantitative phase imaging especially for TIE phase retrieval microscopy which is realized by using only several object field intensities at multiple axially displaced planes, ETL is used as key scanning component. Combining with a traditional microscope, TIE phase retrieval technique has become a competitive and promising tool in the study of bio-science [99-101].

In this study, we apply ETL (type model: EL-10-30-C) as adaptive optical component. The ETL is shown in Fig. 3.1.

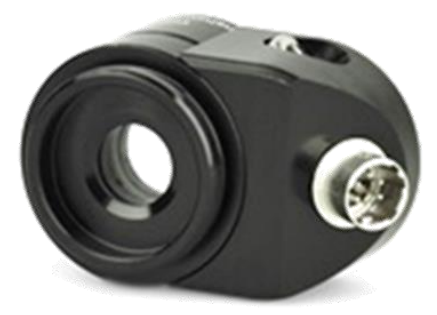

Figure 3.1 Outlook of the ETL

The mechanical specifications are listed in table 3.1. We can see that the ETL is designed with many advantages such as light weight, compact structure, standard connection ports, and long lifecycles. 
Table 3.1 Mechanical specifications.

\begin{tabular}{lll}
\hline \multicolumn{1}{c}{ Mechanical specifications } & & $\mathrm{mm}$ \\
\hline Clear aperture & 10 & $\mathrm{~mm}$ \\
External diameter & 30 & $\mathrm{~mm}$ \\
Thickness & 24.6 & $\mathrm{~g}$ \\
Weight & 34.0 & $\mathrm{~mm}$ \\
Connector length & 100 & \\
Lifecycles (10-90\% sinusoidal) & $>1^{`} 000^{`} 0000^{\prime} 000$ & \\
Temperature sensor \& memory & Yes (SE97B) & \\
\hline
\end{tabular}

Of course, the electrical specifications are also very important to an electrical component.

Table 3.2 lists the electrical specifications of the ETL. From the table, we can see that the response time is less than $2.5 \mathrm{~ms}$ which is fast enough to ensure real-time processing in digital holography.

Table 3.2 Electrical specifications.

\begin{tabular}{lll}
\hline \multicolumn{1}{c}{ Electrical specifications } & & \\
\hline Absolute maximum voltage & 5 & $\mathrm{~V}$ \\
I2C supply voltage & 3.3 (nominal), 3.6 (maximum) & $\mathrm{V}$ \\
Nominal control current with Lens Driver 4 & 0 to 250 & $\mathrm{~mA}$ \\
Absolute max. control current & 0 to 400 & $\mathrm{~mA}$ \\
Power consumption & 0 to 0.9 (nominal), 0 to 2.0 (absolute max.) & $\mathrm{W}$ \\
Response time (10\%-90\% step) & $<2.5$ & $\mathrm{~ms}$ \\
Settling time (typical at $\left.30^{\circ} \mathrm{C}\right)$ & 15 (rectangular step), 6 (controlled step) & $\mathrm{ms}$ \\
\hline
\end{tabular}

\subsection{Calibration of ETL}

\subsubsection{Digital holographic microscope for measurement of ETL}

The schematic of the experimental arrangement is shown in Fig. 3.2. A simple MachZehnder interferometer is employed for measurement. Light from the laser source is spatial filtered to get an ideal point light source. The light is collimated to form a beam with planar wave, and the beam is then split by BS1 into two beams: one beam is 
transmitted through the ETL and magnified by an MO, which forms the object beam; the other beam serves as the reference beam. The two beams then meet and interfere at BS4 with a small angular offset between them. A Field Lens (FL) is used to reshape the image size. The interference pattern between the two beams is captured and recorded by a CCD camera at the image plane.

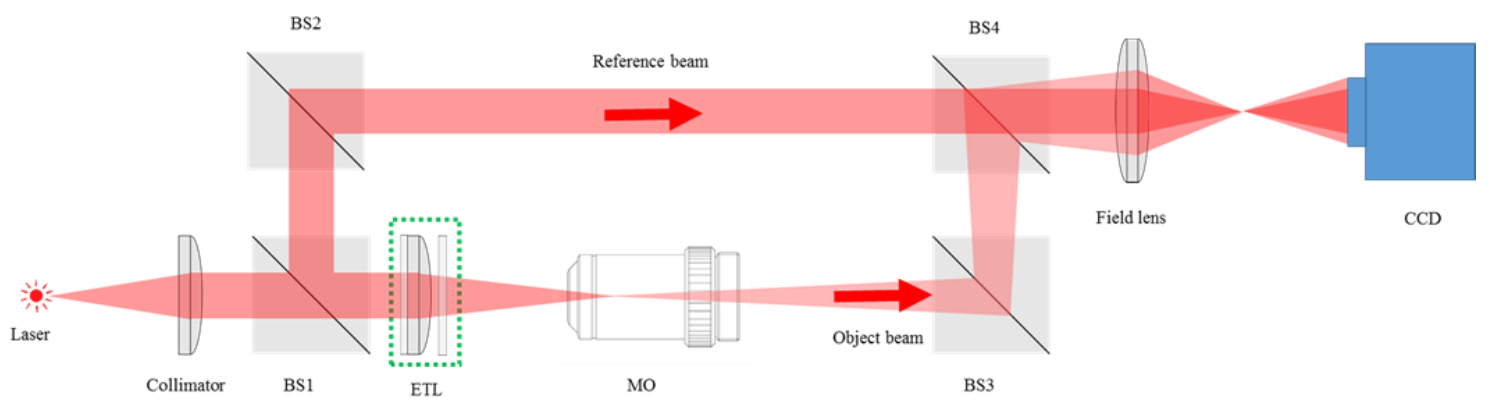

Figure 3.2 Schematic of Mach-Zehnder interferometer for measuring the ETL.

Numerical reconstruction follows ASM in the section 2.3.3(c). The phase of the ETL is then expressed as

$$
\varphi(x, y)=\tan ^{-1} \frac{\operatorname{Re}\left[E_{O}(x, y, d)\right]}{\operatorname{Im}\left[E_{O}(x, y, d)\right]}
$$

The height of ETL membrane surface is obtained with the phase

$$
h(x, y)=\frac{\lambda}{2 \pi\left(n_{E T L}-1\right)} \varphi(x, y)
$$

where $n_{E T L}$ is the refractive index of the ETL.

In order to demonstrate the proposed method experimentally, the Mach-Zehnder interferometer shown in Fig. 3.2 was used. A 660nm solid laser was used as light source. ETL was positioned in the testing arm of the interferometer and object beam was collected by a $2.5 \mathrm{X}$ Olympus MO. The beam in the other arm served as the reference beam and interfered with object beam to generate an interferogram, namely digital 
hologram. To reshape the digital hologram size, an FL with $50 \mathrm{~mm}$ focal length was introduced. The digital hologram was then recorded by an 8-bit Imaging Source USB monochromatic CCD camera. The resolution of CCD camera is $1280 \times 960$ pixels, with pixel size $4.65 \mathrm{um} \times 4.65 \mathrm{um}$. The distance between the tunable lens and $\mathrm{MO}$ is $26 \mathrm{~mm}$. The distance of MO to field lens is $255 \mathrm{~mm}$, and that of FL to CCD camera is $200 \mathrm{~mm}$.

The ETL mode is Optotune EL-10-30-C-LD. The driven current for tunable lens varies from $0 \mathrm{~mA}$ to $290 \mathrm{~mA}$. In the measurement, $10 \mathrm{~mA}$ incremental was applied in driving the ETL; in Fig. 3.3, we select 10mA incremental for display. A wavefront mask without any sample in the system was taken to compensate for the system aberrations. In the measurement, the reconstructed wavefront subtracted the mask before any further calculation. The tilt and piston were then removed from the wavefront by a fast Zernike polynomials fitting with $3^{\text {rd }}$ order terms. In Fig. 3.3, the images are normalized to the same scale to make variances more comparable. A jet colormap is used to render different wavefront values. In a full range, the wavefront value in the center of the ETL varies from 25rad to 70rad with driven current increasing in the experiment. 


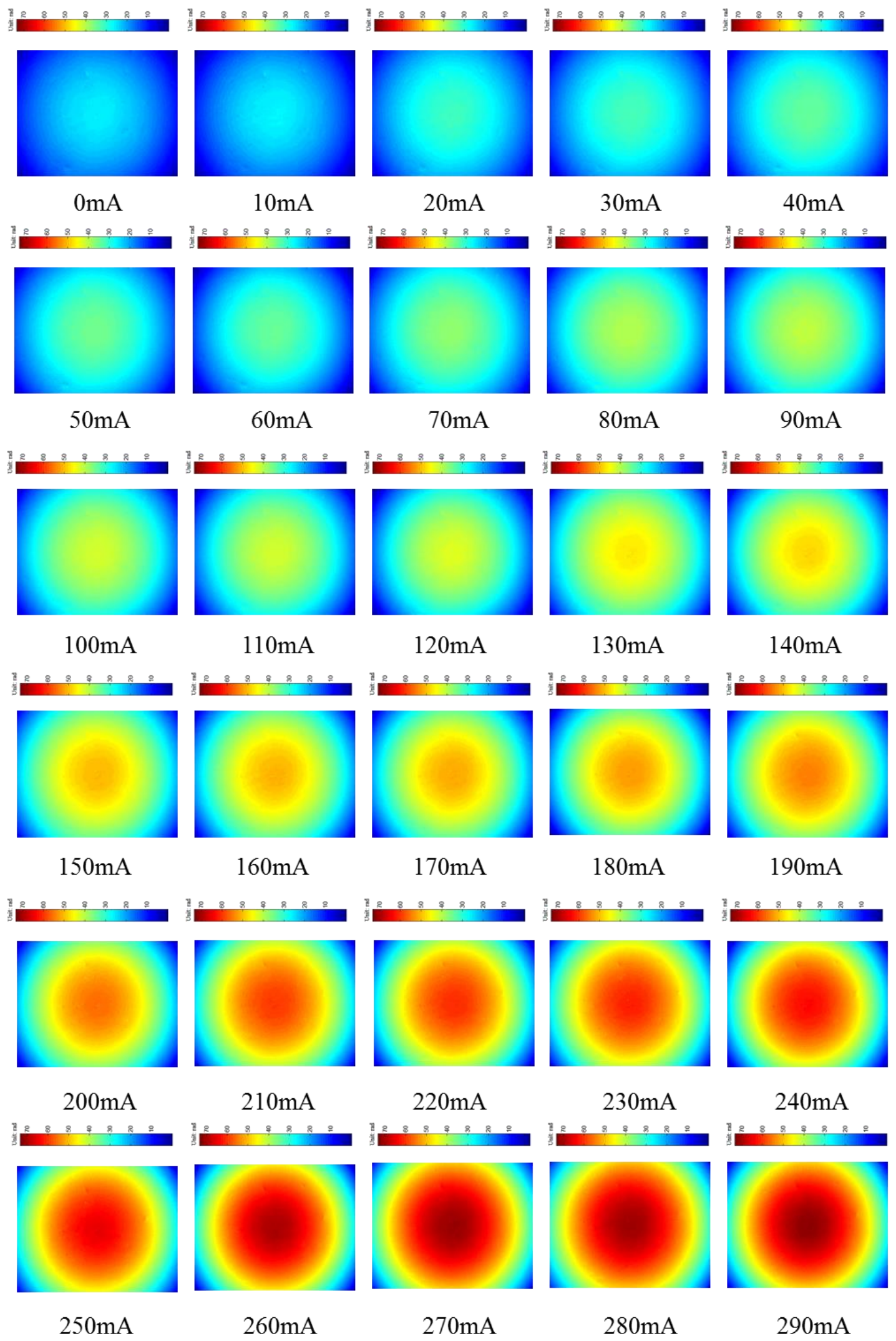

Figure 3.3 ETL wavefront images under different driven current values. 


\subsubsection{Radius calculation by least squares method}

The tomography of ETL can be calculated by Eq. 3.2 (the refractive index of ETL is 1.3). Here, what we obtained is a central part of the ETL surface. We assume it as a spherical crown in Figure 3.4. A Hough transform is performed first to find out the circle center ( $x_{0}$, $\left.y_{0}\right)$. Then the circle center $\mathrm{O}$ is taken as the original point of the coordinate. The top of the spherical crown is located at point $\mathrm{E}$, and the $z$-axis passes points $\mathrm{O}$ and $\mathrm{E}$ with upside as the positive direction. The spherical crown center is assumed to be point G.

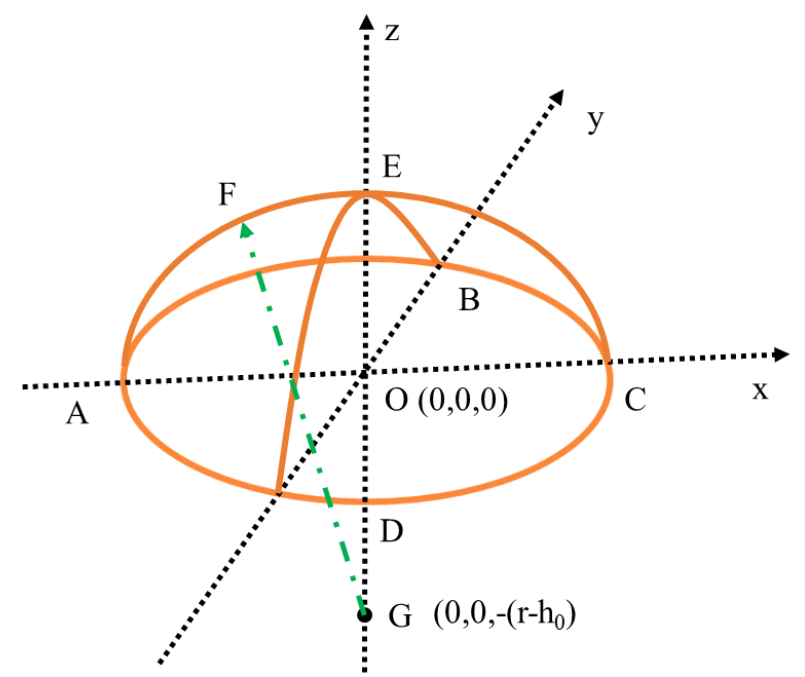

Figure 3.4 Spherical crown model of the ETL surface.

To set up the model with exact meaning, parameters are awarded in the following ways. The height of spherical crown $\mathrm{OE}=\mathrm{h}_{0}$ is calculated by binning $2 \times 2$ pixels around the center $\mathrm{E}$. The center of the spherical crown $\mathrm{G}$ is expressed as $\left(0,0,-\mathrm{r}+\mathrm{h}_{0}\right)$, where $\mathrm{r}$ is the radius of the spherical crown. We set up a mathematical model with the variable of spherical radius $\mathrm{r}$. The measured wavefront data is defined as $\left(x_{i}, y_{i}, z_{i}\right)$, and the deviation of fitting is $\sigma^{2}$. Least squares method is used to fit the wavefront by setting spherical radius as variable

$$
\sigma^{2}=\sum_{i=1}^{n}\left\{\left(x_{i}-x_{0}\right)^{2}+\left(y_{i}-y_{0}\right)^{2}+\left[z_{i}-\left(h_{0}-r\right)\right]^{2}-r^{2}\right\}^{2}
$$


In order to get extreme value of $\sigma^{2}$, differential is taken on the both sides of the Eq. 3.3

$$
\frac{\partial \sigma^{2}}{\partial r}=\frac{\partial\left[x_{i}^{2}+y_{i}^{2}+\left(z_{i}-h_{0}+r\right)^{2}-r\right]^{2}}{\partial r}=0
$$

Thus, the spherical radius $\mathrm{r}$ can be obtained by solving the equation:

$$
r=\frac{\sum_{i=1}^{n}\left[x_{i}^{2}+y_{i}^{2}+\left(z_{i}-h_{0}\right)^{2}\right]}{2 \sum_{i=1}^{n}\left(h_{0}-z_{i}\right)^{2}}
$$

Figure 3.5 shows the radii of ETL at different currents by proposed fitting method. In Fig. 3.5, we know that the radius of ETL is nonlinear versus the driven current.

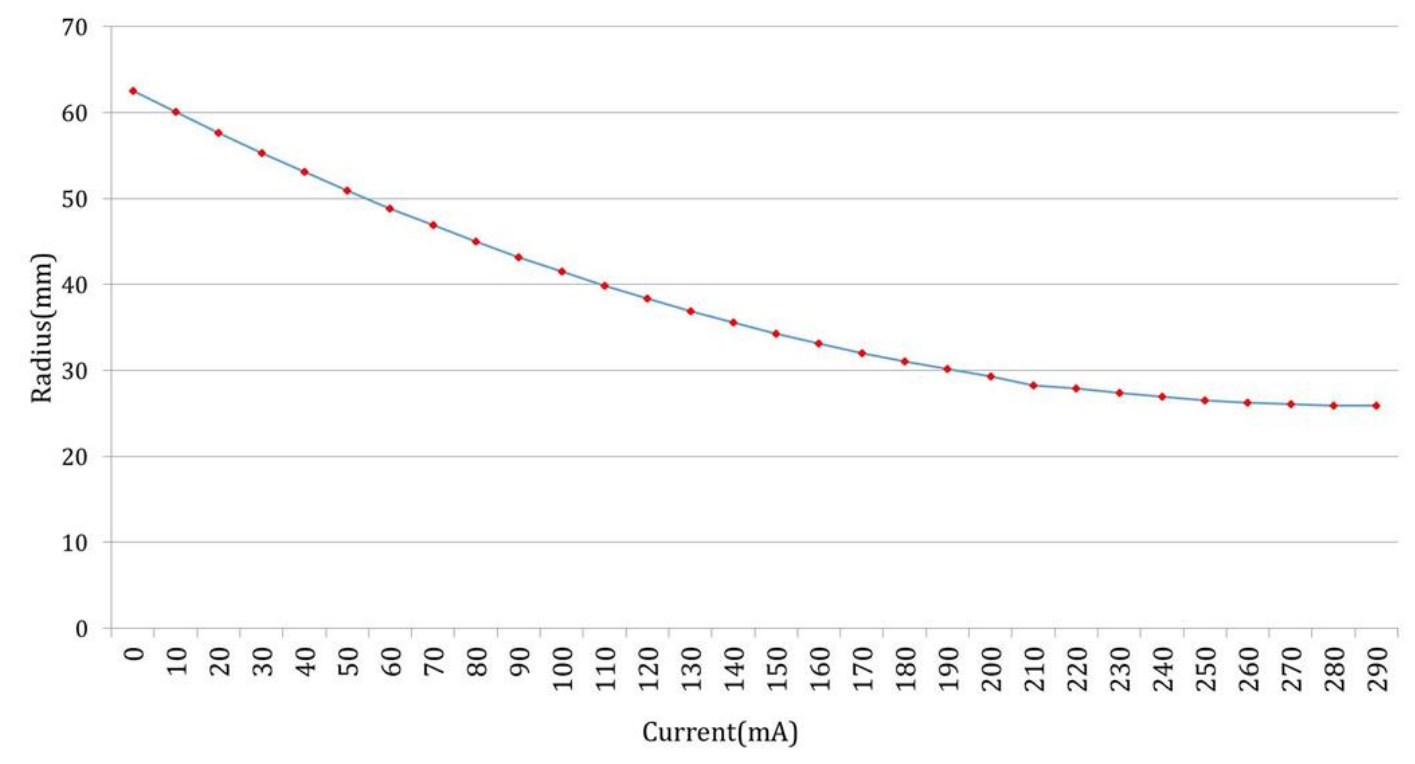

Figure 3.5 ETL radius variation versus driven current changes.

\subsubsection{Focal length calculation by Zemax}

Responding ETL simulation is set up in Zemax 2013. The lens editor of ETL is shown in Fig. 3.6, and the responding 2D layout is demonstrated in Fig. 3.7. Surface 6 represents the adaptive surface in ETL. Through changing the radius at surface 6, it is easy to obtain the focal length by quick focus function in Zemax. The focal length here is calculated 
from surface 6 to surface 11. One must note that the cover glass has different refractive index with air, the effective propagation distance just equals to cover glass thickness times its refractive index (1.5168). When the radius under different driven current are applied, the focal length curve based on radius from LSM was obtained and shown in Fig. 3.8. The focal length curve is also nonlinear against driven current. This behavior further requires calibration and proper mathematical model for practical applications to avoid the error introduced by the nonlinearity of ETL.

\begin{tabular}{|c|c|c|c|c|c|c|c|c|c|c|}
\hline$\Delta$ & Sur & f:Type & Comment & Radius & Thickness & Material & Coating & Semi-Diameter & Conic & TCE $\times 1 \mathrm{E}-6$ \\
\hline 0 & OBJECT & Standard $\mathbf{~}$ & & Infinity & Infinity & & & 0.000 & 0.000 & 0.000 \\
\hline 1 & STOP & Standard $\mathbf{v}$ & & Infinity & 10.000 & & & $3.000 \mathrm{U}$ & 0.000 & 0.000 \\
\hline 2 & & Standard $\mathbf{v}$ & Housing & Infinity & 0.000 & & & $15.000 \mathrm{U}$ & 0.000 & 0.000 \\
\hline 3 & (aper) & Standard $\mathbf{v}$ & & Infinity & 1.150 & & & $5.000 \mathrm{U}$ & 0.000 & 0.000 \\
\hline 4 & (aper) & Standard $\mathbf{~}$ & Container glass front & Infinity & 0.500 & BK7 & & $5.000 \mathrm{U}$ & 0.000 & - \\
\hline 5 & (aper) & Standard $=$ & Container glass back & Infinity & $3.341 \mathrm{E}$ & OL1024_UV_VIS_NIR & & $5.000 \mathrm{U}$ & 0.000 & - \\
\hline 6 & (aper) & Standard $\mathbf{r}$ & Adaptive surface EL-10-30-C & $-25.903 \mathrm{~V}$ & $4.409 \mathrm{E}$ & & & $5.500 \mathrm{U}$ & 0.000 & 0.000 \\
\hline 7 & (aper) & Standard $\mathbf{}$ & Cover glass front & Infinity & 0.500 & BK7 & & $5.500 \mathrm{U}$ & 0.000 & - \\
\hline 8 & (aper) & Standard $=$ & Cover glass back & Infinity & 2.000 & & & $5.500 \mathrm{U}$ & 0.000 & 0.000 \\
\hline 9 & (aper) & Standard $=$ & Holder ring & Infinity & 4.300 & & & $5.500 \mathrm{U}$ & 0.000 & 0.000 \\
\hline 10 & (aper) & Standard & & Infinity & 0.000 & & & $7.000 \mathrm{U}$ & 0.000 & 0.000 \\
\hline 11 & & Standard $\mathbf{r}$ & Housing & Infinity & 74.586 & & & $15.000 \mathrm{U}$ & 0.000 & 0.000 \\
\hline 12 & IMAGE & Standard $\mathbf{~}$ & & Infinity & - & & & $5.000 \mathrm{U}$ & 0.000 & 0.000 \\
\hline
\end{tabular}

Figure 3.6 Lens editor of ETL in Zemax.

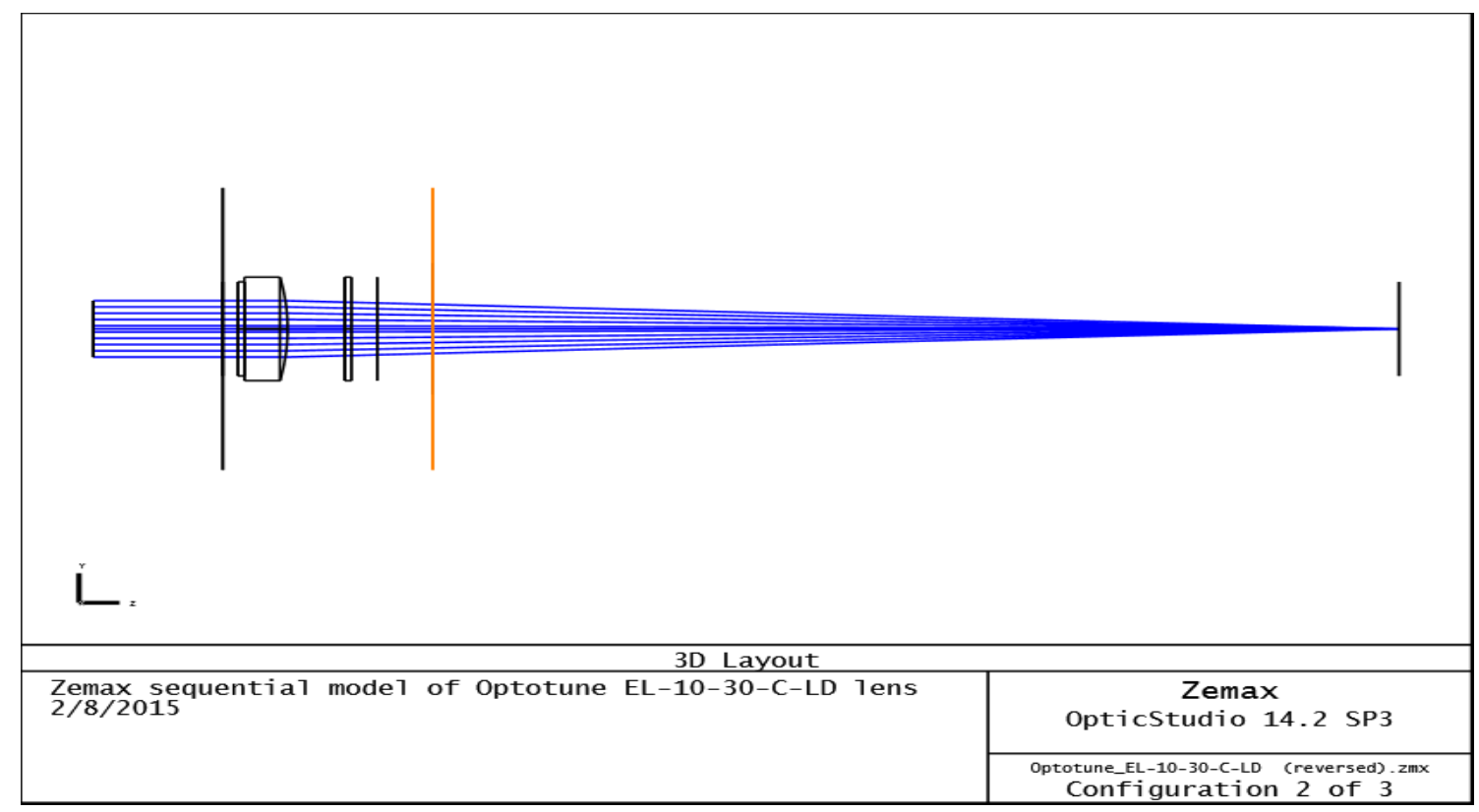

Figure 3.7 The 2D layout of ETL in Zemax. 


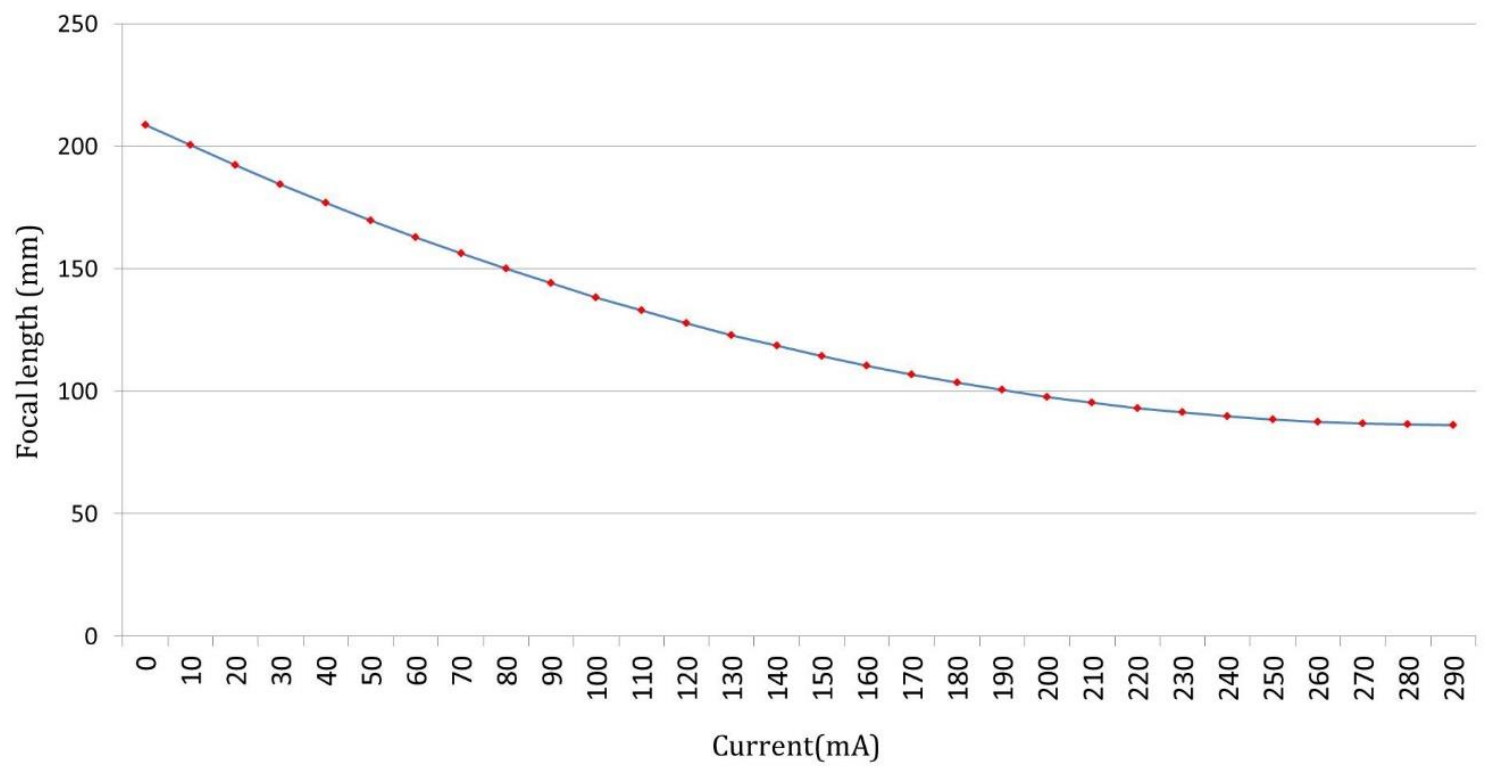

Figure 3.8 ETL focal length variation versus driven current changes.

\subsubsection{Mathematic modeling for tunable lens and its combination with offset lens}

When we obtain a series of focal lengths from Fig. 3.8, we assume it to be a second order curve. Applying a quadratic fitting, the expression with 95\% confidence is as follows.

$$
f_{E T L}(x)=0.001478 x^{2}-0.8505 x+208.6
$$

To demonstrate the fitting more visually, Fig. 3.9 is drawn to show the difference between the fitting curve and the raw data. We can see that the curve is in good agreement with the experimental data. The expression is able to describe the change regularity of ELT focal length. 


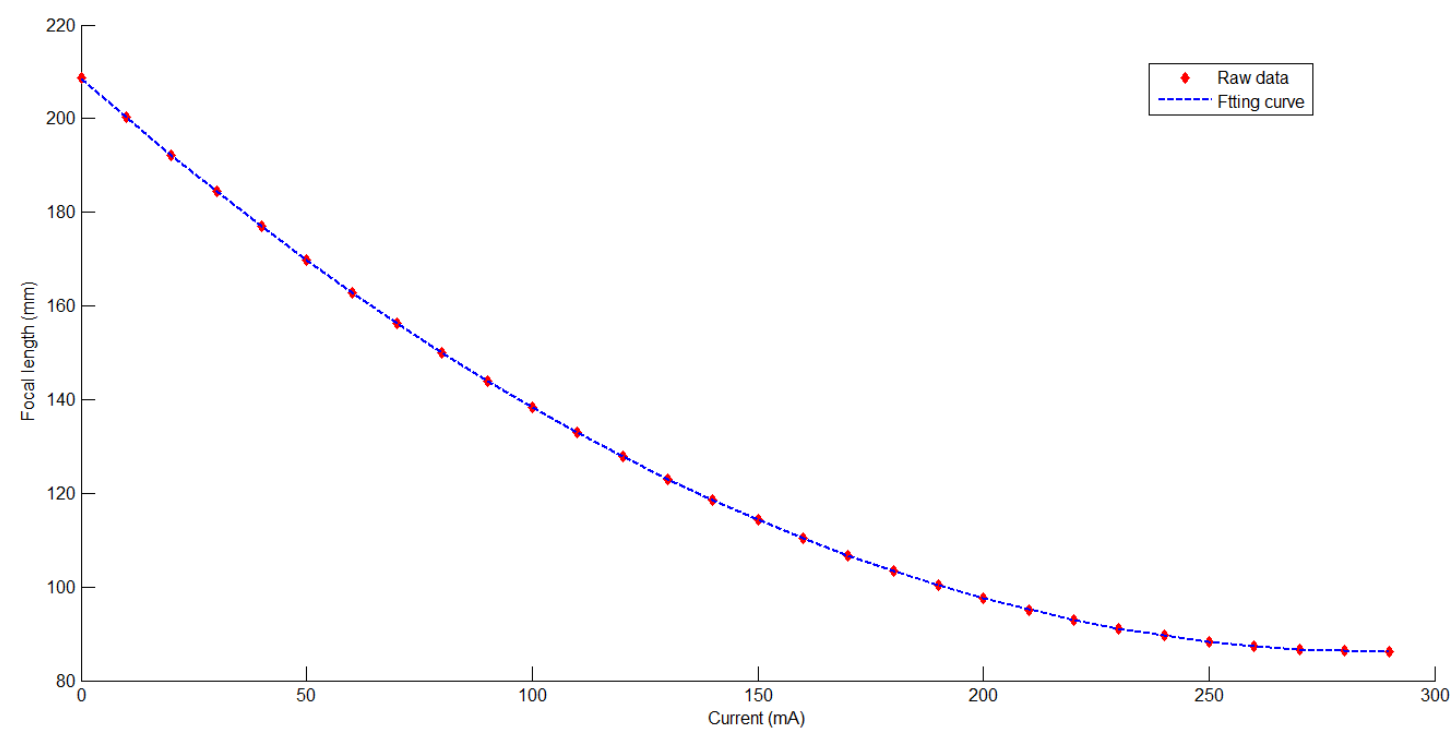

Figure 3.9 Fitting curve and raw data of ETL's focal lengths.

Modeling of the combination of tunable and offset lenses was also deduced to predict its performance rule. Combining with offset lens in tandem arrangement, the distance between them was very small, we took them as in contact; effective focal length was computed by thin lens combination in geometrical optics [102].

$$
\frac{1}{f}=\frac{1}{f_{E T L}}+\frac{1}{f_{O L}}-\frac{d_{L}}{f_{E T L} f_{O L}}
$$

where $d_{L}$ is the distance between ETL and OL. Here the distance is very small to the product of $f_{E T L} \cdot f_{O L}$, thus we ignore the third term on the right side in Eq. 3.7. The focal length of OL is $-150 \mathrm{~mm}$. By substituting the tunable lens focal length expression in Eq. 3.6 into Eq. 3.7, we obtained $\mathrm{x}=80.03 \mathrm{~mA}$, the lens combination would act as flat glass. To verify our prediction, we used setup in section 3.2.1 to test the wavefront of ELT and OL again. When the current was $80 \mathrm{~mA}$, we found in Fig. 3.10 the phase image is uniform and flat. This means our model works well with the practical situation. 


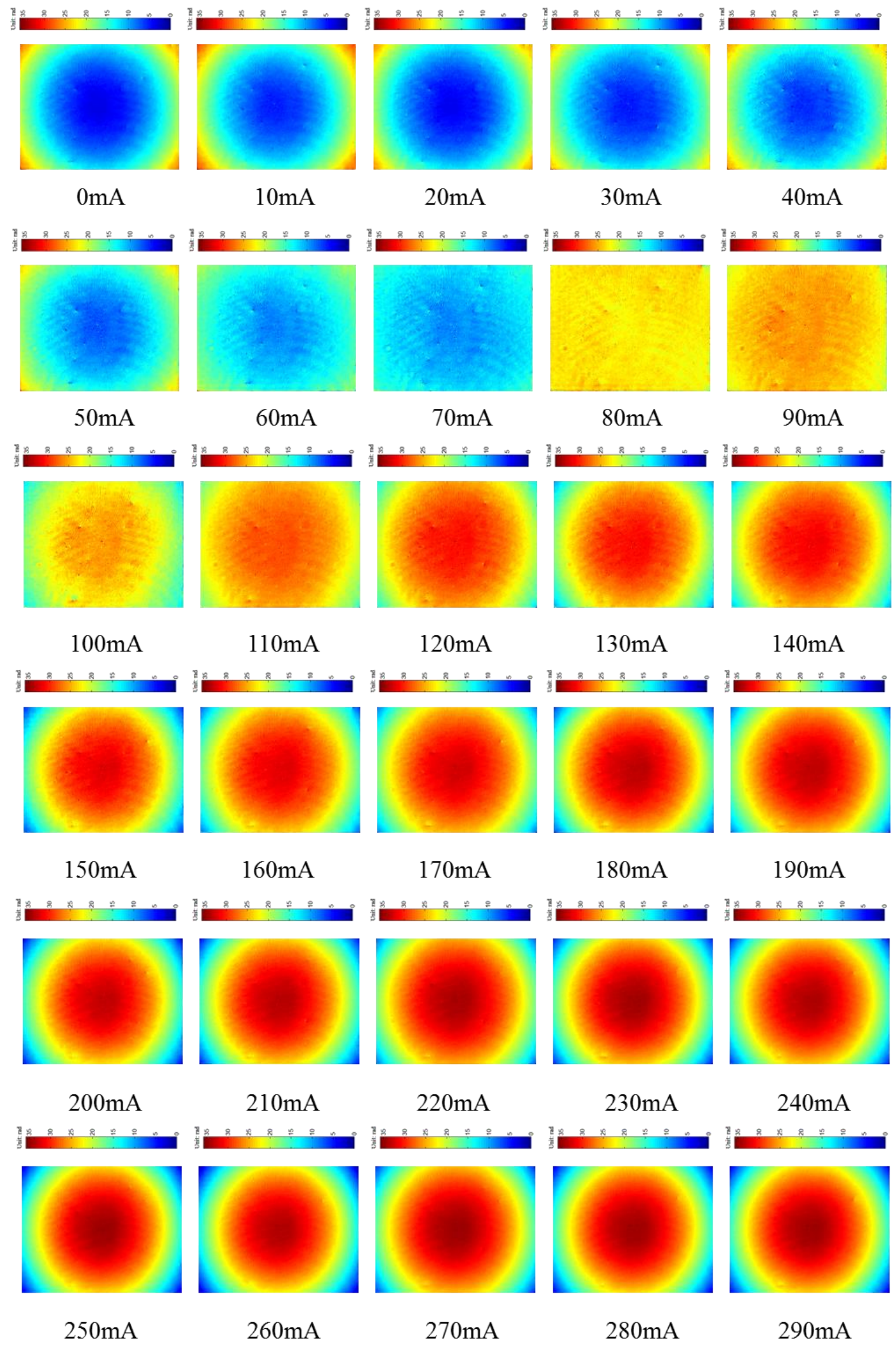

Figure 3.10 Wavefront images of ETL and OL combination under different driven current values. 


\subsection{Principle of digital holography with ETL to measure aspheric surface}

Figure 3.11 shows the schematic of the Mach-Zehnder interferometer for the measurement of aspheric lens. A collimated laser beam is split by BS1 into two beams. One beam goes through the ETL and is then normally incident on the aspheric lens surface under test. Since the beam passing through the aspheric lens diverges very fast, a triplet is introduced to reshape the beam. The other beam serves as the reference beam and interferes with object beam at BS4 with a small angular offset between them. The small angular offset is often manually adjusted to ensure the first order spectrum can be separated from the zero order spectrum in frequency domain. The image is resized by a relay imaging lens and captured by an imaging sensor which is often CCD camera.

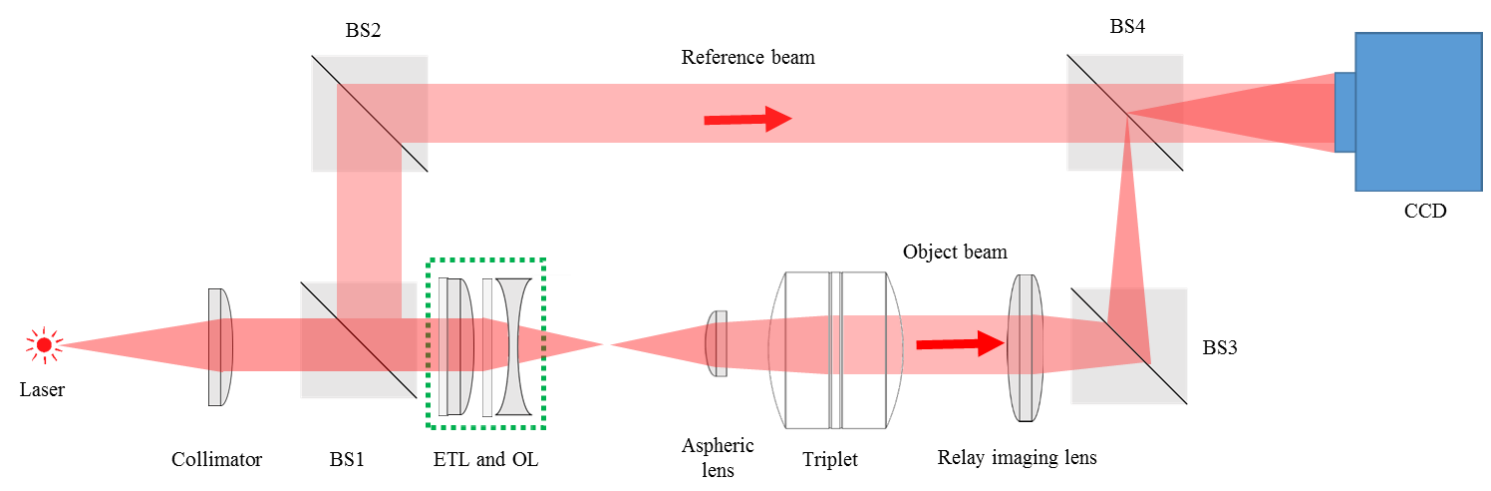

Figure 3.11 Schematic of experimental configuration.

Again, the numerical reconstruction is the same as described in section 2.3.3(c). Through the reconstruction, the phase in the measurement is thus available. The essence of the proposed method is to decompose the large gradients of the asphere into two resolvable surfaces, and to measure them separately. Beyond that, the system aberrations need to be determined beforehand so as not to influence the final result. A double-exposure approach is proposed for the absolute phase measurement. In the first exposure, the 
aspheric lens with thin lens combination of ETL and OL is measured as shown in Fig. 3.11. The ETL drive current is adjusted to minimize the density of the interference fringe in the digital hologram. Once the fringes can be resolved, the digital hologram is recorded and processed to give the reduced phase of the aspheric lens. In the second exposure, the aspheric lens is removed, and a second digital hologram is recorded. The absolute phase of the aspheric lens can thus be retrieved from these two phases.

$$
\varphi_{A L}(x, y)=\varphi_{\text {reduced_AL }}(x, y)+\varphi_{E T L}(x, y)
$$

where $\varphi_{A L}$ is the absolute phase of the aspheric lens. $\varphi_{\text {reduced } A A L}$ and $\varphi_{E T L}$ are the unwrapped phases of the reduced aspheric lens and the ETL, respectively.

Finally, the topography of the aspheric lens can be expressed as

$$
h_{A L}(x, y)=\frac{\lambda}{2 \pi\left(n_{A L}-1\right)} \varphi_{A L}(x, y)
$$

where $h_{A L}$ is the height on aspheric lens surface. $n_{A L}$ is the refractive index of aspheric lens.

\subsection{Experiment and results}

\subsection{1. $4.5 \mathrm{~mm}$ diameter aspheric lens testing}

In experiment, an aspheric lens primarily used for collimating the beam emerging from an optical fiber was tested. The diameter and focal length of the aspheric lens are $4.5 \mathrm{~mm}$ and $2.5 \mathrm{~mm}$, respectively. A $660 \mathrm{~nm}$ solid state laser was used as light source and the focal length of the triplet and the relay imaging lens was $40 \mathrm{~mm}$ and $100 \mathrm{~mm}$, respectively. The 
digital hologram was recorded by an 8-bit monochromatic CCD camera with $1280 \times 960$ pixels with a pixel size of $4.65 \mu \mathrm{m} \times 4.65 \mu \mathrm{m}$. The distance between ETL and OL thin lens combination and the aspheric lens was $305 \mathrm{~mm}$ while the distance of the aspheric lens to triplet was $5 \mathrm{~mm}$. The triplet was $75 \mathrm{~mm}$ from the relay imaging lens which in turn was 90mm from the CCD. As the fitting algorithm was developed in polar co-ordinates, all the phases were apodized into a circular aperture for evaluation. Through calibration with a USAF 1951 resolution target, the diametric field of view was $3.033 \mathrm{~mm}$.

Figure 3.12(a) shows the interference fringes generated by the aspheric lens without any current applied to the ETL. The fringes cannot be resolved by the imaging system. Next current was applied to the ETL to reduce the density of the fringes and for this example a current of $123.2 \mathrm{~mA}$ gave resolvable fringes. The wrapped phase of reduced aspheric lens is shown in Fig. 3.12(b). In second exposure, the phase without the aspheric lens is measured and displayed in Fig. 3.12(c). It can be seen that both wrapped phases are resolvable and can be easily unwrapped.

(a)

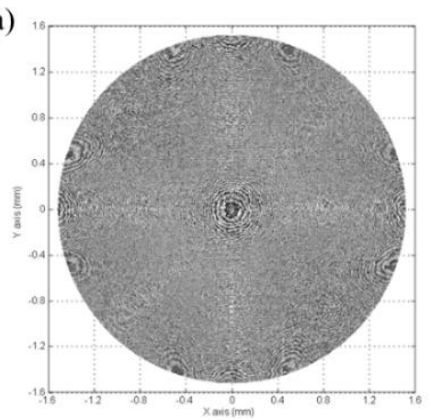

(b)

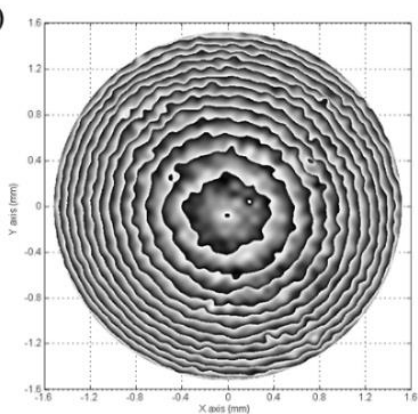

(c)

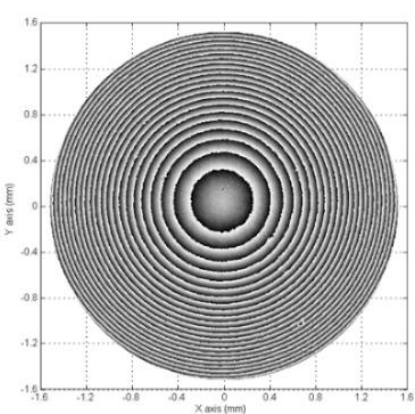

Figure 3.12 The wrapped phase of (a) asphere which cannot be resolved, (b) the reduced aspheric lens and (c) the ETL decomposed by (a) which can now be analyzed.

Figures 3.13(a) and (b) are the unwrapped phases of the reduced aspheric lens and the ETL respectively. The absolute phase aspheric lens can then be recovered as shown in Fig. 3.13(c). After implementing the fitting procedure, the aspheric parameters were 
obtained and compared with factory data in table 3.3. It can be seen that the parameters are in good agreement. The reconstructed phase based fitted parameters is displayed in Fig. 3.13(e) with the residue shown in Fig. 3.13(f). In this case, the mean value of the residue is $1.29 \mathrm{e}-17 \mu \mathrm{m}$, while the standard deviation of the residue is $0.042 \mu \mathrm{m}$. The variance in the residue is mainly caused by random noise and irregularity of optical surface in the whole system. The standard deviation coincides with our system error level which is ten nanometers. While the extreme small mean value clearly demonstrates the average statistical characteristic of the random distribution of noise. In order to compare the goodness of the fitting, data along the $480^{\text {th }}$ line in Figure 6(c) and (e) were plotted in Fig. $3.13(\mathrm{~g})$. It can be noted that two plots also show a good agreement.

Table 3.3 Aspheric lens parameters comparison.

\begin{tabular}{|c|c|c|c|c|c|c|c|}
\hline & $\begin{array}{l}\text { Aspheric } \\
\text { parameters }\end{array}$ & A04 & A06 & A08 & A10 & $\begin{array}{l}\text { Radius } \\
(\mathrm{mm})\end{array}$ & $\begin{array}{l}\text { Conic } \\
\text { Constant }\end{array}$ \\
\hline \multirow{3}{*}{ Test 1} & Factory & $3.255 \mathrm{E}-3$ & $8.501 \mathrm{E}-5$ & $-2.227 \mathrm{E}-5$ & $\sim$ & -2.3289 & -0.8814 \\
\hline & Fitted & $3.287 \mathrm{E}-3$ & $8.572 \mathrm{E}-5$ & $-2.225 \mathrm{E}-5$ & $\sim$ & -2.3289 & -0.8781 \\
\hline & Factory & $1.954 \mathrm{E}-5$ & $-1.756 \mathrm{E}-8$ & $2.597 \mathrm{E}-11$ & $-2.414 \mathrm{E}-14$ & -22.09 & -2.271 \\
\hline Test 2 & Fitted & $1.999 \mathrm{E}-5$ & $-1.872 \mathrm{E}-8$ & $2.874 \mathrm{E}-11$ & $-2.933 \mathrm{E}-14$ & -22.09 & -2.232 \\
\hline
\end{tabular}


(a)

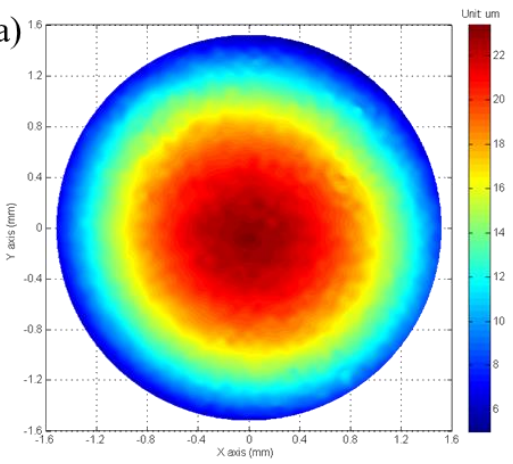

(c)

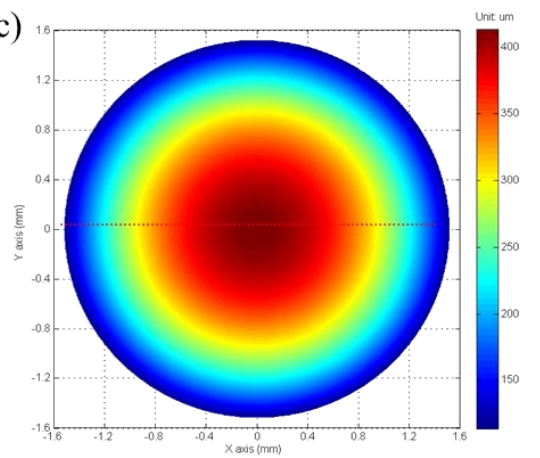

(e)

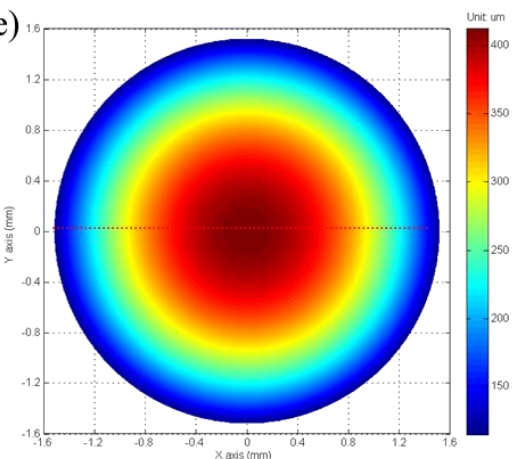

(b)

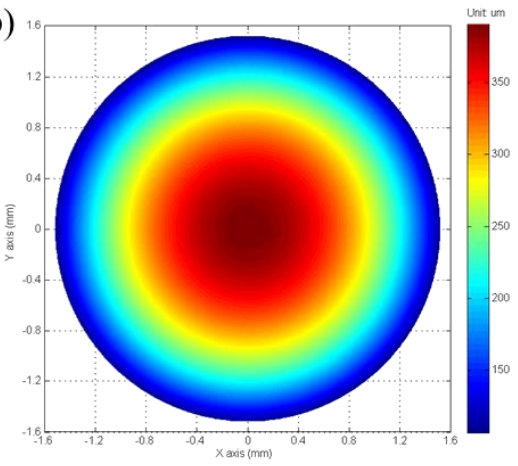

(d)

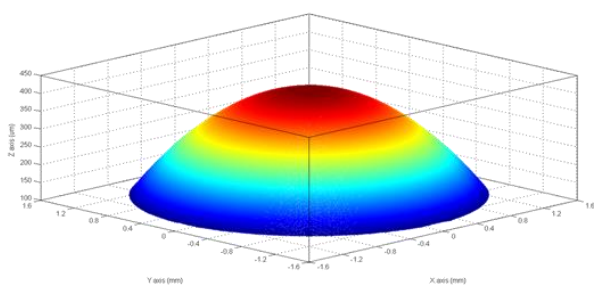

(f)

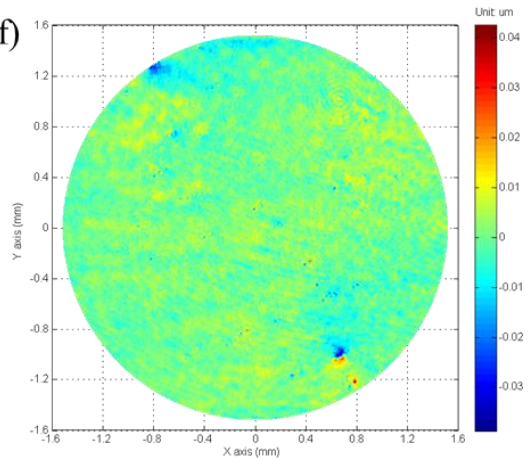

(g)

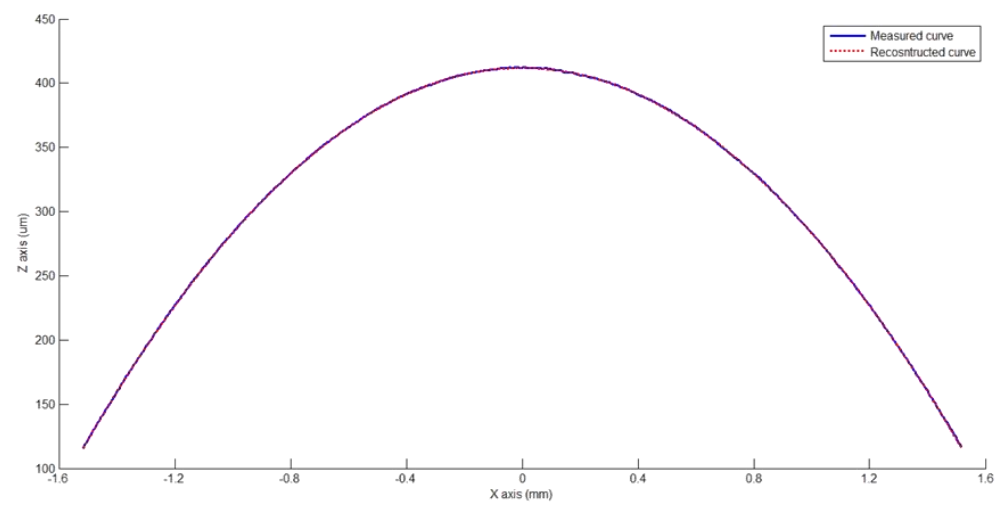

Figure 3.13 Testing 1. Unwrapped phases of (a) the ETL, (b) the reduced aspheric lens, and (c) the aspheric lens. (d) 3D rendering for the phase of the aspheric lens. (e) Reconstructed phase by fitted parameters. (f) Residue phase between measured phase (c) and reconstructed phase (e). (g) Profile comparison of 480th line between measured and reconstructed curves. 


\subsection{2. $25 \mathrm{~mm}$ diameter aspheric lens testing}

The second aspheric lens tested was a high precision aspheric lens (model: KPA16) from Newport, with $25.00 \mathrm{~mm}$ diameter and $37.50 \mathrm{~mm}$ focal length, respectively. With $25.00 \mathrm{~mm}$ clear aperture, its NA is approximately 0.33 . The distance of thin lens combination to the aspheric lens was $285 \mathrm{~mm}$ and the distance of the aspheric lens to the triplet was $50 \mathrm{~mm}$. The relay imaging was $65 \mathrm{~mm}$ from the lens triplet and the distance between the relay lens and CCD camera was $80 \mathrm{~mm}$. The drive current applied to the ETL was $9.3 \mathrm{~mA}$. The field of view was calibrated again, and the normalized aperture diameter was $9.65 \mathrm{~mm}$ for this example.

Figures 3.14(a) and (b) are the reconstructed phases of the reduced aspheric lens and the ETL, respectively. The absolute phase of the aspheric lens is displayed in Fig. 3.14(c). After fitting, the aspheric parameters were obtained and compared to factory values in table 3.3. It can be seen that as the coefficient goes weaker with the term order increasing, the accuracy of fitting also goes lower. For weak aspheric coefficients, many factors such as fabrication, measurement scheme, system noise, fitting algorithm and computing errors would affect the final result. The reconstructed phase by fitted parameters is shown in Fig. 3.14(e), and the residue is shown in Fig. 3.14(f). The mean value of the residue is $2.5188 \mathrm{e}-16 \mu \mathrm{m}$, while the standard deviation of the residue is $0.064 \mu \mathrm{m}$. Again, data along the $480^{\text {th }}$ line in Fig. 3.14(c) and (e) are plotted in Fig. 3.14(g). In the plot, fitting result and the experimental data are in good agreement. However, the irregularity in the plot fluctuates a bit stronger than that in the previous testing due to the larger aperture which introduces more noise. 
(a)

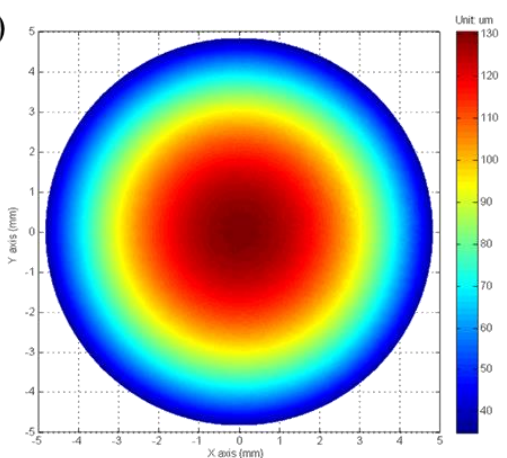

(c)

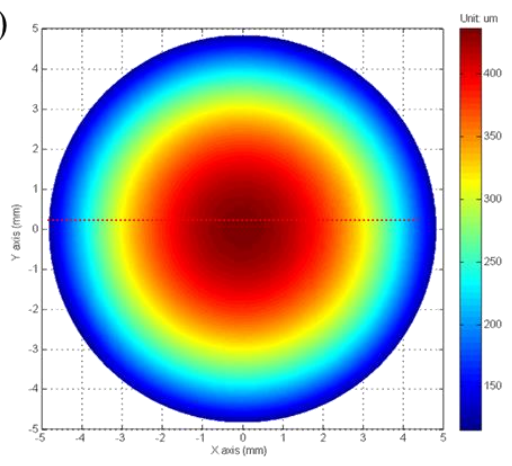

(e)

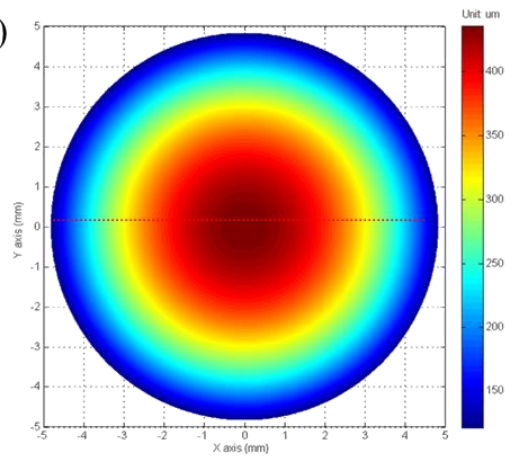

(b)

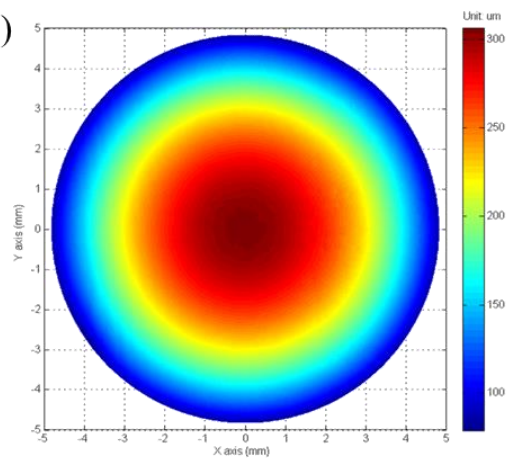

(d)

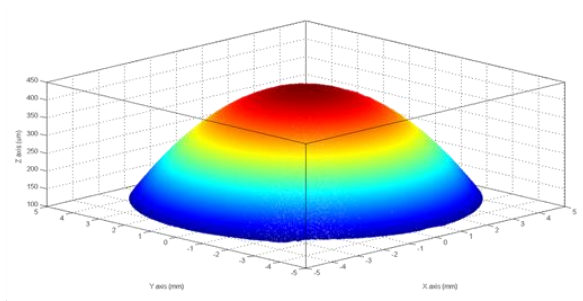

(f)

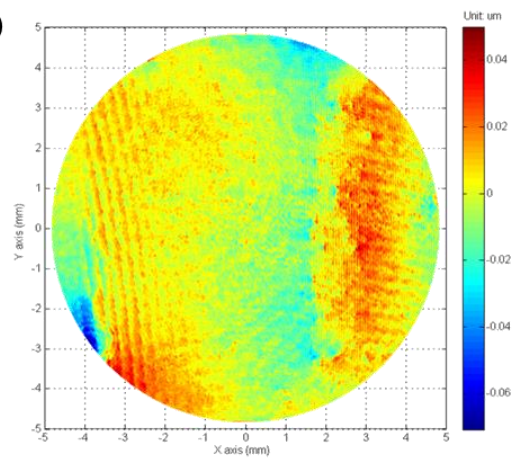

(g)

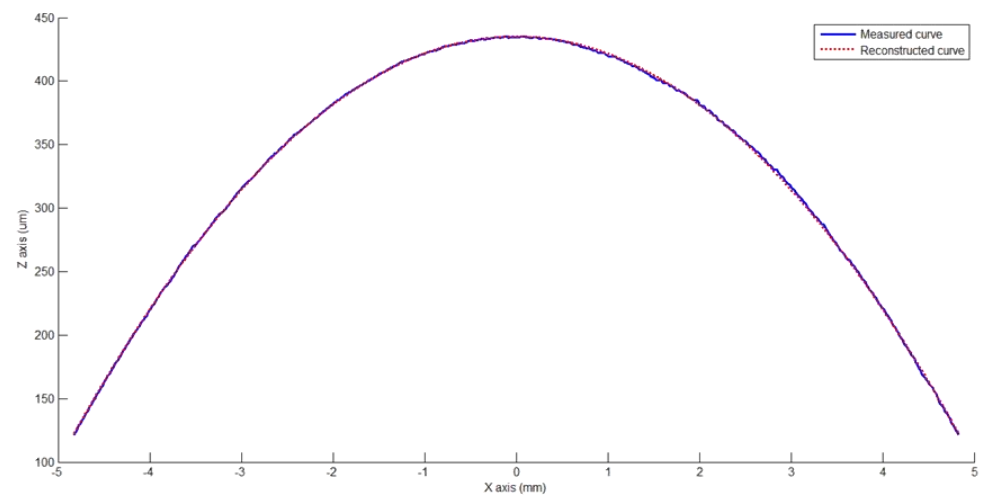

Figure 3.14 Testing 2. Unwrapped phases of (a) the ETL, (b) the reduced aspheric lens, and (c) the aspheric lens. (d) 3D rendering for the phase of the aspheric lens. (e) Reconstructed phase by fitted parameters. (f) Residue phase between measured phase (c) and reconstructed phase (e). (g) Profile comparison of 480th line between measured and reconstructed curves. 


\subsection{Chapter summary}

In summary, a new aspheric lens measurement method is presented. The method introduces adaptive optical component-ELT as phase corrector to measure aspheric lens in digital holography. The narrow range of ETL's NA limits its flexibility. To overcome this drawback, an OL is introduced. The thin lens combination greatly extends the tuning power and thus improves the range of NA.

Unlike the CGH approach, the proposed method doesn't require the aspheric lens to identically match the NA of the system. The thin lenses combination is used to compensate the spherical sag in aspheric lens, thus reducing the high gradients on the aspheric lens surface. By double-exposure technique, the proposed method permits absolute phase measurement. Furthermore, quantitative characterization is available which is often required in finish evaluation during design or fabrication.

To verify the validity of the proposed method, aspheric lenses with different NA have been tested. Through parametric and visual comparisons, the experimental results are in good agreement with factory data. As all of the samples applied in this study are planoconvex structure, the Mach-Zehnder interferometer was thus employed. For aspheric lens without plano-surface, Tyman-Green or Fizeau interferometer is recommended as they are more suitable for testing reflective surface. The proposed method can also be extended to reflective surface measurement. 


\section{DUAL-WAVELENGTHS DIGITAL HOLOGRAPHY TO MEASURE ASPHERIC SURFACE}

In this chapter, the axial resolution of single wavelength digital holography is discussed. The axial resolution is limited to half wavelength in two neighboring pixels. Therefore, single wavelength digital holography is not capable of resolving discontinuities that are larger half wavelength. DWDH is explored to solve the problem. As the most important aspect in DWDH, the synthetic wavelength can greatly improve the system capability of resolving discontinuities. Once the measurement is done, the most important work in DWDH is phase unwrapping. Three existing dual-wavelength phase unwrapping methods are reviewed and modified for implementation. Moreover, we propose a linear programming dual-wavelength phase unwrapping method. Simulation of step structures is applied to all above phase unwrapping methods. In order to test the robustness of these methods, different levels of Gaussian noise are put on the step structures. The performances of these methods are analyzed in kinds of PV values, mean values, and standard deviations. At last, experiments on MEMS, cylindrical lens and aspheric lens are done to verify DWDH.

\subsection{Dual-wavelength digital holography}

In digital holography, the axial resolution is limited in $\lambda / 2$ within two neighboring pixels with respect to the sampling theorem [103]. Considering 760nm wavelength which is close to the upper limit in visible light, the largest jump resolvable by the system can only reach 380nm. In many applications, such as measurement of MEMS, micro-optics, or aspheric lens, may fail due to high gradients on the sample's surface often exceed the 
systematic resolution limit. Different strategies have been investigated to solve this problem. Phase shifting is often used in interferometry and also available in digital holography [104-106]. However, the phase shifter is very expensive, and will definitely increase the system cost. Furthermore, being a scanning process, phase shifting is timeconsuming. Alternatively, by synthesizing two different wavelengths into a much larger beat wavelength, DWDH greatly improves the capability of resolving higher jumps. As a result, it has been successfully applied in different samples measurement, such as cells, BGA, porous coal, fluid lens, and so on [107-113].

Many efforts have been done in developing DWDH in past few years. By multiplexing, single shot dual-wavelength digital holographic microscopy which can do real-time reconstruction was mostly investigated and reported $[114,115]$. By introducing polarizer for beam separation, single shot dual wavelength digital holography can be also realized [116-118]. The structure with single shot is obviously the most convenient for operation, but at the same time, the structure becomes complex and the cost increases. In general, digital holographic system for single wavelength is also available for DWDH by doubleexposure without increasing any cost. On the other hand, concerns also pay on phase unwrapping. The phase retrieved by digital holography also suffers from modulo $2 \pi$ ambiguities. In DWDH, the phase is retrieved from two wrapped phases. Therefore, the phase unwrapping problem turns to find the optimal integers to match two phases. Three methods, calculated distance equal, fractional fringes, and equivalent wavelength were discussed and compared in [119]. A simple phase unwrapping strategy was proposed in [120]. A more efficient linear regression phase unwrapping method was described and applied in cancer cells measurement [121]. 


\subsection{Modification of existing phase unwrapping algorithms}

In digital holography, once the phase is obtained, its phase height map is thus retrievable according to different configurations. In reflection, it gives

$$
h(x, y)=\frac{\varphi(x, y)}{4 \pi} \lambda
$$

Here $\lambda$ is the wavelength of the laser. For transparent object, it gives

$$
h(x, y)=\frac{\varphi(x, y)}{2 \pi\left(n-n_{0}\right)} \lambda
$$

where $n-n_{0}$ is the refractive index difference between the object and the surrounding medium.

Assume the object under testing is a reflective surface and is measured by doubleexposure with two different wavelengths. Moreover, the overall object height is larger the both wavelengths. Next, the height map can be written as

$$
\begin{aligned}
& h(x, y)=\frac{\varphi_{1}(x, y)}{4 \pi} \lambda_{1}+m(x, y) \lambda_{1} \\
& h(x, y)=\frac{\varphi_{2}(x, y)}{4 \pi} \lambda_{2}+n(x, y) \lambda_{2}
\end{aligned}
$$

where $m(x, y)$ and $n(x, y)$ are the unknown nonnegative integer pair at point $(x, y)$. We assume $\lambda_{2}$ is greater than $\lambda_{1}$. Subtracting the second equation from the first in Eq. 4.3 gives

$$
h(x, y)=\frac{\lambda_{1} \lambda_{2}}{\lambda_{2}-\lambda_{1}} \cdot\left[\frac{\varphi_{1}(x, y)-\varphi_{2}(x, y)}{4 \pi}+m(x, y)-n(x, y)\right]
$$

where $\Lambda=\frac{\lambda_{1} \lambda_{2}}{\lambda_{2}-\lambda_{1}}$ is the beat wavelength. Eq. 4.4 gives expression of the height in the form of beat wavelength. We can see that the unknowns are two integer numbers $m(x, y)$ 
and $n(x, y)$. Once they are confirmed, the height map is accessible by any expression in Eq. 4.3 or 4.4 .

Since the phases for two single wavelengths are wrapped and there are discontinuities exceeding the axial resolution of single wavelength digital holography, dual-wavelength phase unwrapping is required before any further application in Eq. 4.1 or 4.2. In order to remove the $2 \pi$ discontinuities, different strategies are proposed.

\subsubsection{Calculated distances equal}

This method makes use of the fact that the measured distance is independent of the wavelength used. If $m(x, y)=n(x, y)+\Delta(x, y)$, it gives

$$
n(x, y) \lambda_{1}+\frac{\varphi_{1}(x, y)}{4 \pi} \lambda_{1}=[n(x, y)+\Delta(x, y)] \lambda_{2}+\frac{\varphi_{2}(x, y)}{4 \pi} \lambda_{2}
$$

where $n(x, y)$, and $\Delta(x, y)$ are integers.

Solving for $n(x, y)$, we have

$$
n(x, y)=\frac{\varphi_{1}(x, y) \lambda_{1} / 4 \pi-\varphi_{2}(x, y) \lambda_{2} / 4 \pi-\Delta(x, y) \lambda_{2}}{\lambda_{1}-\lambda_{2}}
$$

We must find $\Delta(x, y)$ such that $n(x, y)$ is an integer. In general there will be more than one $\Delta(x, y)$ that will solve the above equation to within the accuracy to which we know the wavelengths and measured fractional fringe. If the approximate length is known, the range for $n(x, y)$ and $\Delta(x, y)$ are known. In the absence of any knowledge concerning the range for $\Delta(x, y)$ the best that can be done is to pick the smallest value of $\Delta(x, y)$ for which the above equation is valid. $\Delta(x, y)$ can be obtained from the following if tolerance 
is an acceptable amount for $n(x, y)$ to differ from being an integer. Consider environmental conditions, a reasonable value for tolerance might be 0.001 .

Given: $\Delta(x, y) \leftarrow 0$

while abs [Fractional Part $(n(x, y))]>$ tolerance

$$
\text { do } \Delta(x, y) \leftarrow \Delta(x, y)+1
$$

end

In the While function $\Delta(x, y)$ will keep increasing by 1 each time through the loop until the fractional part of $n(x, y)$ differs by less than the tolerance.

This method has the lowest accuracy because errors in $\varphi_{1}(x, y)$ and $\varphi_{2}(x, y)$ are scaled by $\lambda /\left(\lambda_{1}-\lambda_{2}\right)$, which can be a large number. Therefore, any small errors in $\varphi_{1}(x, y)$ and $\varphi_{2}(x, y)$ are magnified in the calculation of $n(x, y)$. This method is easy to understand, but it will lead to the least accuracy.

Because the While loop does end up until the condition is satisfied. This may lead to two possible problems in implementation: the loop will never stop because there is no convergence point; another situation is that even if there is a convergence point, it needs long time searching which is time-consuming. Any of above situations is a disaster to practical application. To make the program more implementable, restrain can be applied to $n(x, y)$. Assume the maximum of the discontinuity is known to limit in one beat wavelength $\Lambda$, the maximal integer for $n(x, y)$ can be written as 


$$
n_{\max }=\operatorname{round}\left(\frac{\Lambda}{\lambda_{2}}\right)
$$

As a result, the While loop can be rearranged as

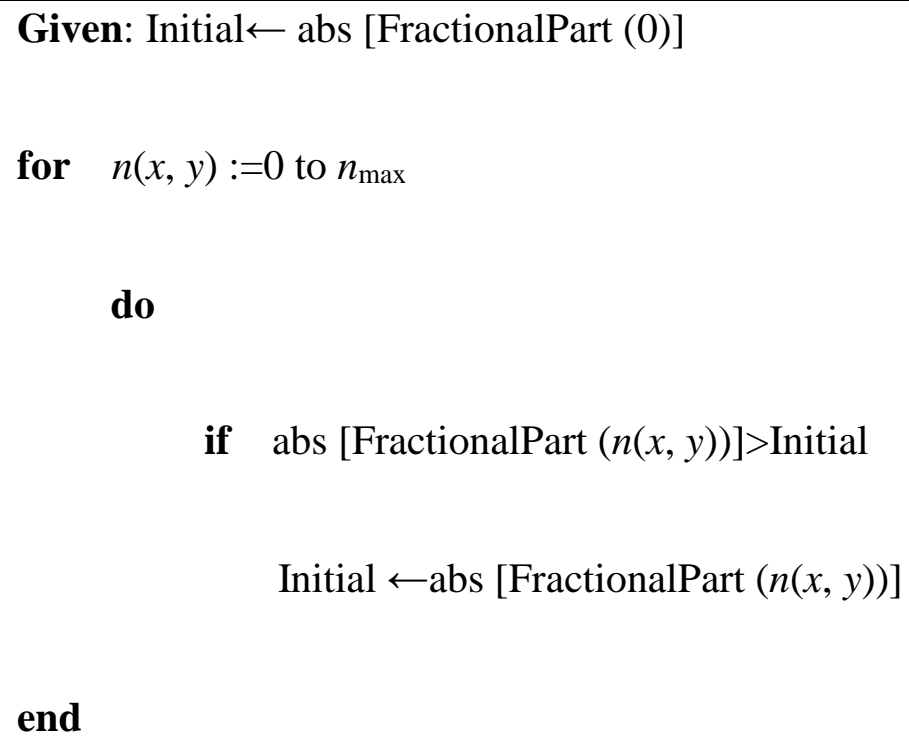

Above program is more feasible than that applies While loop. The program in fact converts an unbounded problem into a bounded one. Of course, one may argue that the actual integer number for $n(x, y)$ may exceed the $n_{\max }$. The boundary applied will be incorrect. In our knowledge, the practical $n_{\max }$ is usually not so large, especially considering the error magnification, the phase unwrapping often fail first before reaching too large $n_{\max }$.

\subsubsection{Fractional fringes}

The second method is basically a computerized version of a method called exact fractions described by Michelson and Benoît in 1895. In the exact fractions approach a length is measured by measuring the excess fractional fringes for two or more wavelengths. It is assumed that the length is known to within a few half wavelengths. The first step is to 
calculate the fractional fringe for wavelength $\lambda_{2}$ from Equation 0 for calculated Optical Path Difference (OPD) of $\lambda_{1}$.

$$
\operatorname{FractionalPart}\left(\lambda_{2}\right)=\text { FractionalPart }\left[\frac{n(x, y) \lambda_{1}+{ }_{1} \varphi_{1}(x, y) \lambda / 4 \pi}{\lambda_{2}}\right]
$$

If we had no error this should be equal to the measured fractional fringe for wavelength $\lambda_{2}$. That is, in the absence of error.

$$
\varphi_{2}(x, y)=\text { FractionalPart }\left[4 \pi \frac{n(x, y) \lambda_{1}+\varphi_{1}(x, y) \lambda_{1} / 4 \pi}{\lambda_{2}}\right]
$$

This equation can be solved for $n(x, y)$, and the OPD can be calculated. In general there will be more than one $\mathrm{n}$ that will solve the above equation to within the accuracy to which we know the wavelengths and measured fractional fringe. If an approximate length is known, the range for $n(x, y)$ is known. Picking the smallest value of $n(x, y)$ for which the above equation is valid and letting tolerance be the acceptable difference between the calculated fractional fringe and the measured fractional fringe we could write

Given: $n(x, y) \leftarrow 0$

while abs [FractionalPart $\left.\left(4 \pi \frac{n(x, y) \lambda_{1}+\varphi_{1}(x, y) \lambda_{1} / 4 \pi}{\lambda_{2}}\right)-\varphi_{2}\right]>$ tolerance

$$
\text { do } n(x, y) \leftarrow n(x, y)+1
$$

end 
In the While function $n(x, y)$ will keep increasing by 1 each time through the loop until the calculated and fractional fringe for $\lambda_{2}$ differ by less than the tolerance.

Above program also has the same problem in computation due to the While loop. Similarly, we rearrange it as

$$
\begin{aligned}
& \text { Given: Initial } \left.\leftarrow \text { abs [FractionalPart }\left(4 \pi \frac{n(x, y) \lambda_{1}+\varphi_{1}(x, y) \lambda_{1} / 4 \pi}{\lambda_{2}}\right)-\varphi_{2}\right] \\
& \text { for } n(x, y):=0 \text { to } n_{\max } \\
& \text { do } \\
& \left.\quad \text { if } \operatorname{abs~[FractionalPart~}\left(4 \pi \frac{n(x, y) \lambda_{1}+\varphi_{1}(x, y) \lambda_{1} / 4 \pi}{\lambda_{2}}\right)-\varphi_{2}\right]>\text { Initial } \\
& \text { Initial } \leftarrow \text { abs }\left[\text { FractionalPart }\left(4 \pi \frac{n(x, y) \lambda_{1}+\varphi_{1}(x, y) \lambda_{1} / 4 \pi}{\lambda_{2}}\right)-\varphi_{2}\right] \\
& \text { end }
\end{aligned}
$$

\subsubsection{Linear regression}

Here, we rewrite Eq. 4.3 for separate reconstruction

$$
\begin{aligned}
h(x, y) & =\frac{\varphi_{1}(x, y)}{4 \pi} \lambda_{1}+m(x, y) \lambda_{1} \\
& =\frac{\varphi_{2}(x, y)}{4 \pi} \lambda_{2}+n(x, y) \lambda_{2}
\end{aligned}
$$

In order to evaluate the unknown integers $m(x, y)$ and $n(x, y)$, the least square of the height difference is applied to search optimal integer match pair of $m(x, y)$ and $n(x, y)$

$$
m(x, y)=\frac{\lambda_{2}}{\lambda_{1}} n(x, y)+\frac{1}{4 \pi}\left[\frac{\lambda_{2}}{\lambda_{1}} \varphi_{2}(x, y)-\varphi_{1}(x, y)\right]
$$


If there is no uncertainty in phase and wavelength measurements, the solution of above linear equation should be exactly two integer values for $m(x, y)$ and $n(x, y)$. Furthermore, unless there is a common divisor between two wavelengths, the range for unambiguous height measurements can be extended to infinity, because there is only one set of integers, $m(x, y)$ and $n(x, y)$, where Eq. 4.10 holds exactly true. When the phase measurement is finished, Eq. 4.11 can be applied to determine the most accurate integer pair of $m(x, y)$ and $n(x, y)$ by rounding

$$
m(x, y)=\operatorname{round}\left\{\frac{\lambda_{2}}{\lambda_{1}} n(x, y)+\frac{1}{4 \pi}\left[\frac{\lambda_{2}}{\lambda_{1}} \varphi_{2}(x, y)-\varphi_{1}(x, y)\right]\right\}
$$

where $n(x, y)$ is such that the difference between Eqs. 4.11 and 4.12 is at the minimum.

In order to make this method more effective, we modify it as

$$
\begin{aligned}
& \text { Given: Initial } \leftarrow\left\{\frac{1}{4 \pi}\left[\frac{\lambda_{2}}{\lambda_{1}} \varphi_{2}(x, y)-\varphi_{1}(x, y)\right]\right\}^{\wedge 2} \\
& \text { for } n(x, y):=0 \text { to } n_{\max } \\
& \text { do } \\
& \text { if }\left[\frac{\lambda_{2}}{\lambda_{1}} n(x, y)+\frac{1}{4 \pi}\left[\frac{\lambda_{2}}{\lambda_{1}} \varphi_{2}(x, y)-\varphi_{1}(x, y)\right]-m(x, y)\right]^{\wedge 2}>\text { Initial } \\
& \text { Initial } \leftarrow\left\{\frac{\lambda_{2}}{\lambda_{1}} n(x, y)+\frac{1}{4 \pi}\left[\frac{\lambda_{2}}{\lambda_{1}} \varphi_{2}(x, y)-\varphi_{1}(x, y)\right]-m(x, y)\right\}^{\wedge 2}
\end{aligned}
$$




\subsection{Principle of linear programing phase unwrapping algorithm}

Here, we rewrite Eq. 4.3 for separate reconstruction

$$
\begin{aligned}
& h_{1}(x, y)=\frac{\varphi_{1}(x, y)}{4 \pi} \lambda_{1}+m(x, y) \lambda_{1} \\
& h_{2}(x, y)=\frac{\varphi_{2}(x, y)}{4 \pi} \lambda_{2}+n(x, y) \lambda_{2}
\end{aligned}
$$

In order to evaluate the unknown integers $m(x, y)$ and $n(x, y)$, the least square of the height difference is applied to search optimal integer match pair of $m(x, y)$ and $n(x, y)$

$$
\min \left[\left(h_{1}-h_{2}\right)^{2}\right]=\min \left\{\left[\frac{\varphi_{1}(x, y) \lambda_{1}-\varphi_{2}(x, y) \lambda_{2}}{4 \pi}+m(x, y) \lambda_{1}-n(x, y) \lambda_{2}\right]^{2}\right\}
$$

Similarly, the equation for transmissive configuration is given as

$$
\min \left[\left(h_{1}-h_{2}\right)^{2}\right]=\min \left\{\left[\frac{\varphi_{1}(x, y) \lambda_{1}-\varphi_{2}(x, y) \lambda_{2}}{2 \pi\left(n-n_{0}\right)}+m(x, y) \lambda_{1}-n(x, y) \lambda_{2}\right]^{2}\right\}
$$

As Eq. 4.15 has two variables, the boundary condition needs to be determined for practical implementation. In conventional methods, one variable is often expressed with another variable. By changing the latter variable value in a linear manner, a simple restrain as in Eq. 4.15 is then put on the former variable to search the optimal value. In such way, the boundary condition is limited in one dimension. In our method, two dimension boundary conditions are applied which searches for global optimal values of both variables at the same time. In fact, boundary conditions must be determined because it may lead to convergence problem which is a complete disaster in implement. The guide line for setting the search boundary is given as follows: firstly, the boundary should be larger than both of single wavelength; secondly, for computing convenience, we take integer times of beat-wavelength as the boundary condition; thirdly, the noise is enlarged 
with the same magnification with respect to the ratio of beat wavelength to single wavelength; last but not the least, one can estimate the object maximum discontinuity and set up the boundary condition. As the maximum of the discontinuity is assumed to be the beat wavelength, the maximal integers for $\lambda_{1}$ and $\lambda_{2}$ can be written as

$$
\begin{aligned}
& m_{\max }=\operatorname{round}\left(\frac{\Lambda}{\lambda_{1}}\right) \\
& n_{\max }=\operatorname{round}\left(\frac{\Lambda}{\lambda_{2}}\right)
\end{aligned}
$$

In order to make this method more effective, we modify it as

$$
\begin{aligned}
& \text { Given: Initial } \leftarrow\left[\frac{\varphi_{1}(x, y) \lambda_{1}-\varphi_{2}(x, y) \lambda_{2}}{4 \pi}\right]^{2} \\
& \text { for } n(x, y):=0 \text { to } n_{\max } \\
& \text { do } \\
& \text { for } m(x, y):=0 \text { to } m_{\max } \\
& \text { do } \\
& \begin{array}{l}
\text { if } \quad\left[\frac{\varphi_{1}(x, y) \lambda_{1}-\varphi_{2}(x, y) \lambda_{2}}{4 \pi}+m(x, y) \lambda_{1}-n(x, y) \lambda_{2}\right]^{2}>\text { Initial } \\
\text { Initial } \leftarrow\left[\frac{\varphi_{1}(x, y) \lambda_{1}-\varphi_{2}(x, y) \lambda_{2}}{4 \pi}+m(x, y) \lambda_{1}-n(x, y) \lambda_{2}\right]^{2}
\end{array}
\end{aligned}
$$

end

end 


\subsection{Simulation}

In order to verify the methods, we simulate four continuous step structures for testing. Consider there are noises in practical measurement, different levels of Gaussian noise are purposely added in the simulation.

First, we generate a matrix for height map. The matrix for this simulation is assumed to be $600 \times 600$ pixels, with pixel size $4.65 \mu \mathrm{m} \times 4.65 \mu \mathrm{m}$. Four continuous steps are designed to $1 / 4,1 / 2,3 / 4$ and 1 of the beat wavelength. The phase is shown in Fig. 4.1(a). The step shapes are all rectangle with $100 \times 200$ pixels and all aligned in the center of the matrix.

In this simulation, we assume the configuration is reflective. Here we apply $532 \mathrm{~nm}$ and $632.8 \mathrm{~nm}$ as light source wavelengths. Therefore, the beat wavelength is $3.34 \mu \mathrm{m}$ which has been significantly extended with respect to each wavelength. The 3D rendering of the step structures is shown in Fig. 4.1(b). According to Eq. 4.1, the phase for two single wavelengths can be calculated since the height map is known. Once the phase is ready, the wrapped phase is readily obtained by MATLAB function. Figures 4.1(c) and (d) shows two wrapped phases converted by the equation. It can be seen that two wrapped phases are modulated with different contrast even at the same place.

In general, there is always noise in the phase measurement. Therefore, we also consider this situation to test the robustness the algorithm. In this simulation, we use Gaussian distribution for the noise in MATLAB [122]. For the distribution of the noise, the variance is from 0 to $10^{-3}$. To evaluate four methods, the residue is obtained by subtracting the height map in Fig. 4.1(b) from the height map recovered by the unwrapped phase. 
(a)

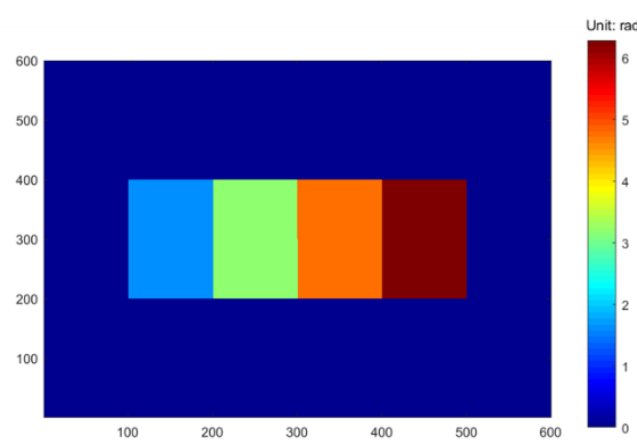

(c)

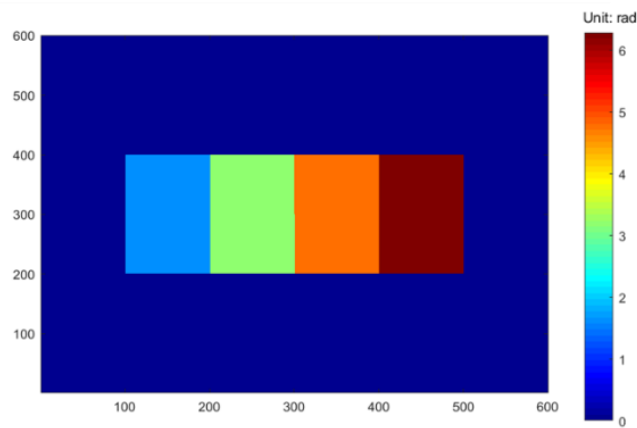

(b)
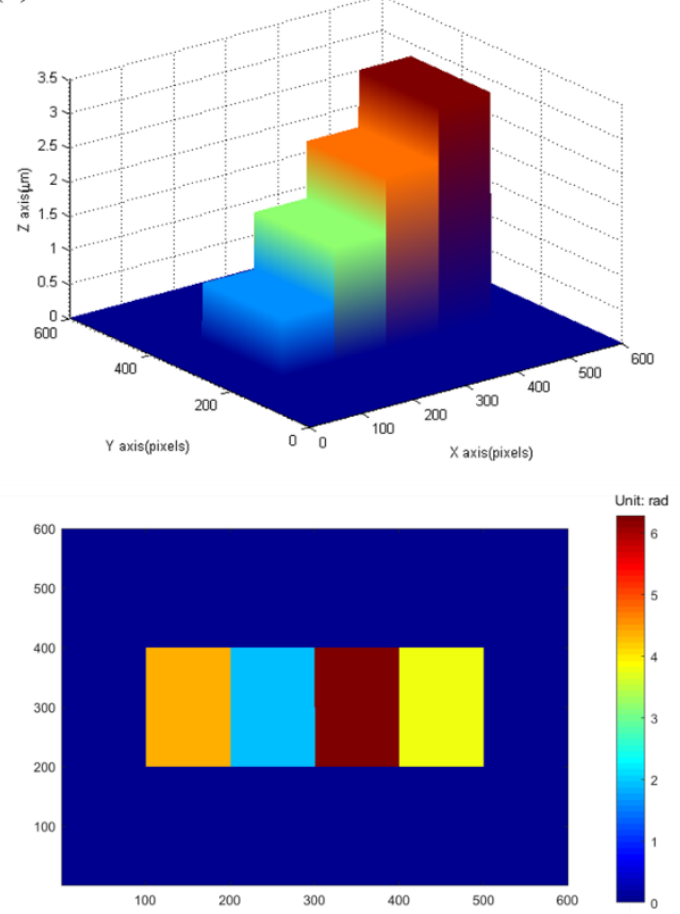

Figure 4.1 (a) The phase with respect to beat wavelength, and its (b) 3D height map, (c) wrapped phase corresponding to $532 \mathrm{~nm}$, (d) wrapped phase corresponding to $632.8 \mathrm{~nm}$.

In order to show the unwrapping results more visually, we display four set of unwrapped phases in Figs. 4.2-4.5 for four phase unwrapping methods, respectively. In each figure, the variance values are selected at $0,10^{-5}, 10^{-4}$ and $10^{-3}$. In Figs. 4.2-4.5, it can be seen that variations in the residue increase with the noise variance increasing. While in each recovered height map, the shapes are correct with respect to the Fig. 4.1(b). It indicates that three modified methods as well as our proposed method are straightforward in phase unwrapping. Moreover, all of them are robust in present of noise. 
(a)

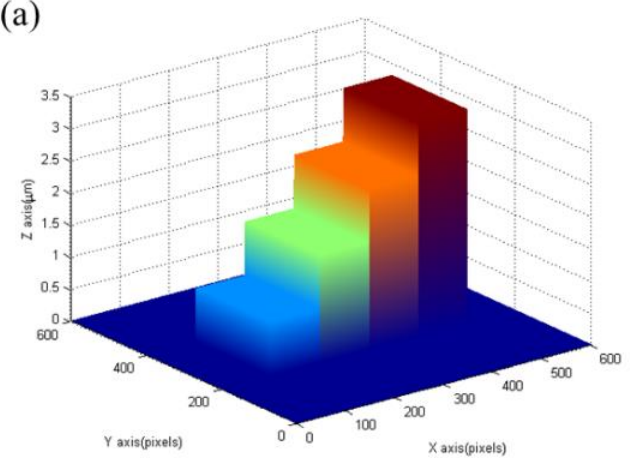

(c)

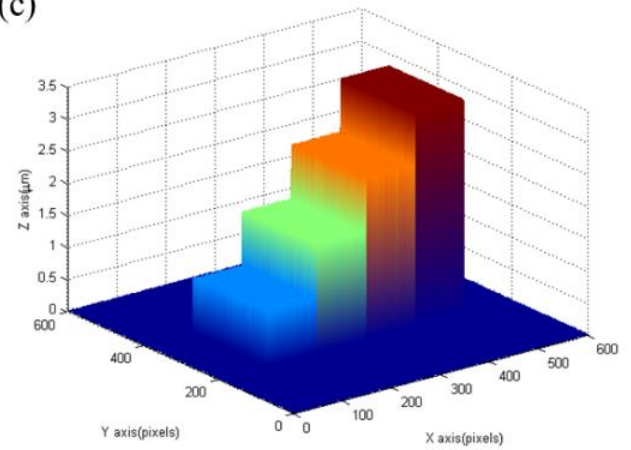

(e)

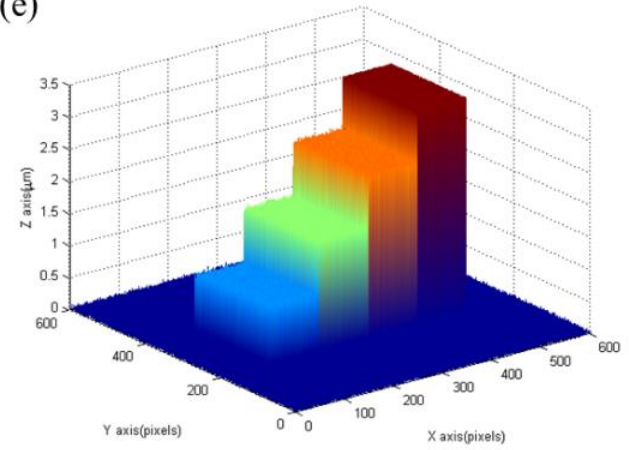

$(\mathrm{g})$

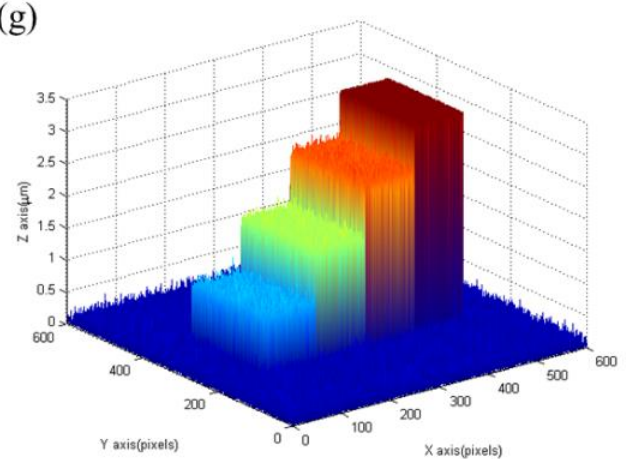

(b)

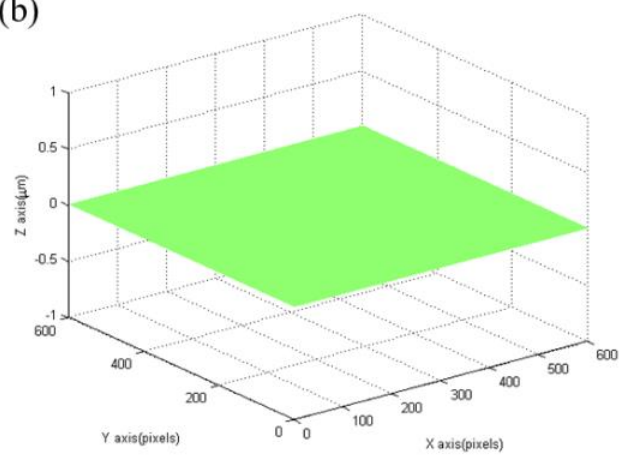

(d)

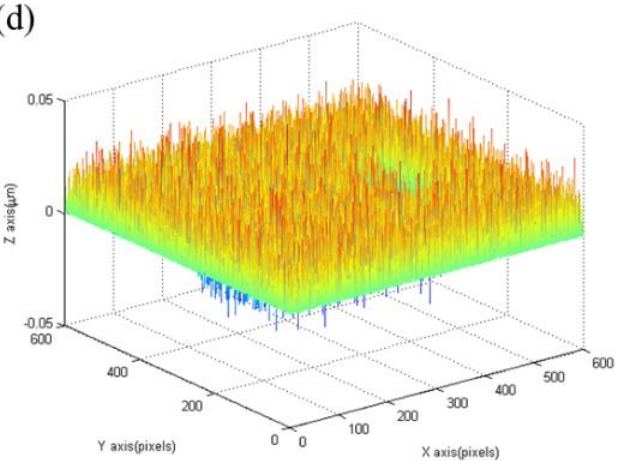

(f)

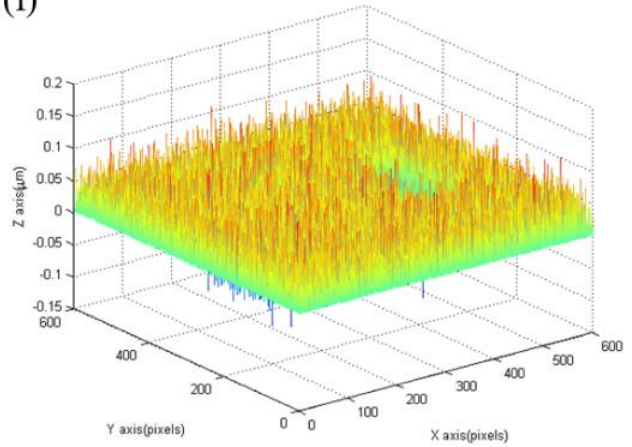

(h)

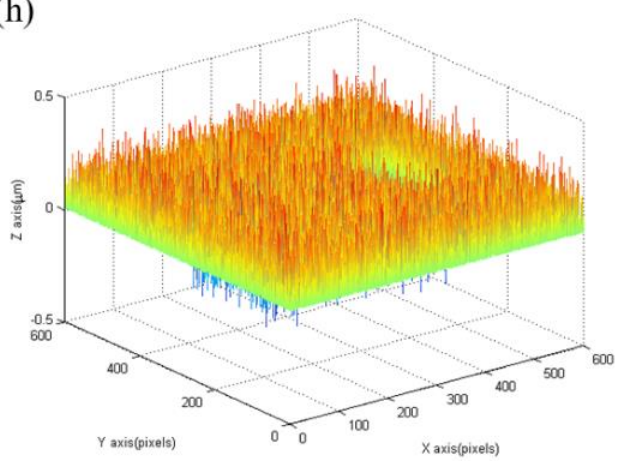

Figure 4.2 Method 1 (a) unwrapped phase when noise variance $=0$, and its (b) residue, (c) unwrapped phase when noise variance $=10^{-5}$, and its (d) residue, (e) unwrapped phase when noise variance $=10^{-4}$, and its (f) residue, (g) unwrapped phase when noise variance $=10^{-3}$, and its (h) residue. 
(a)

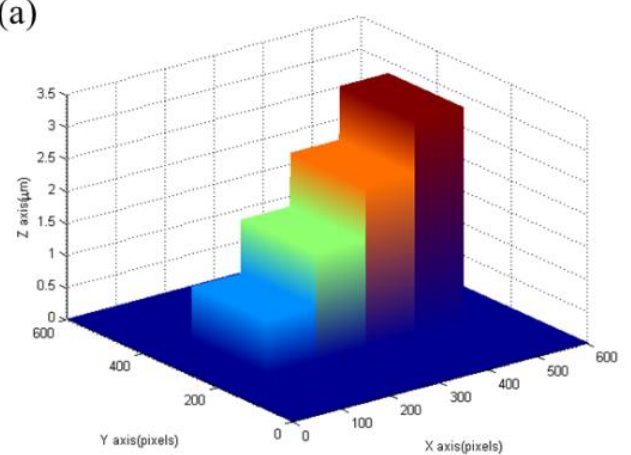

(c)

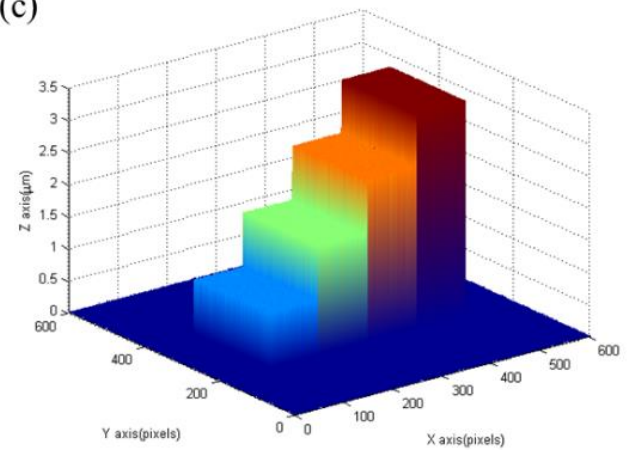

(e)

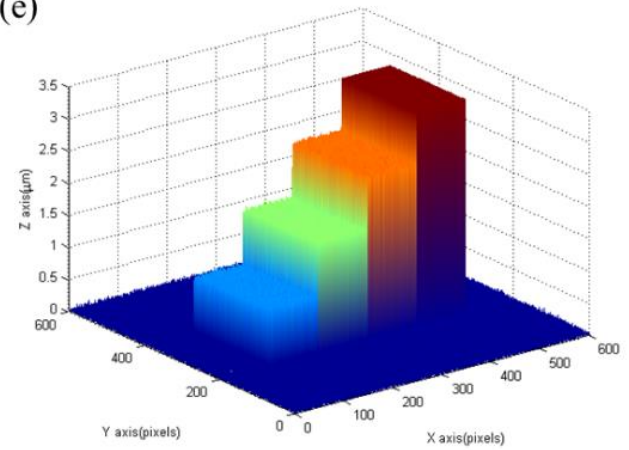

(g)

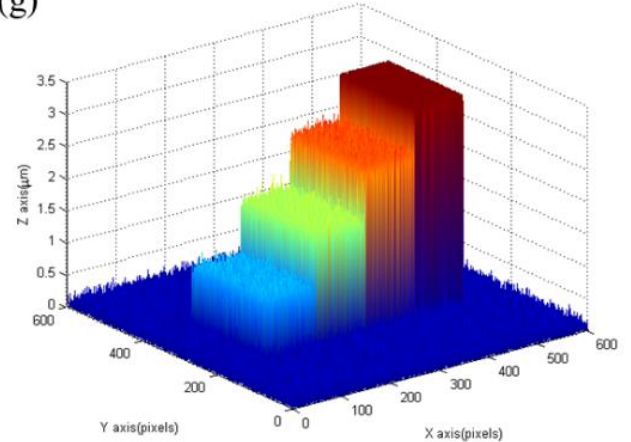

(b)

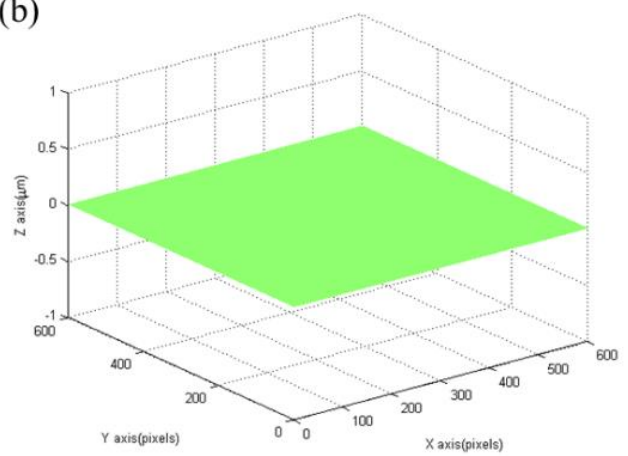

(d)

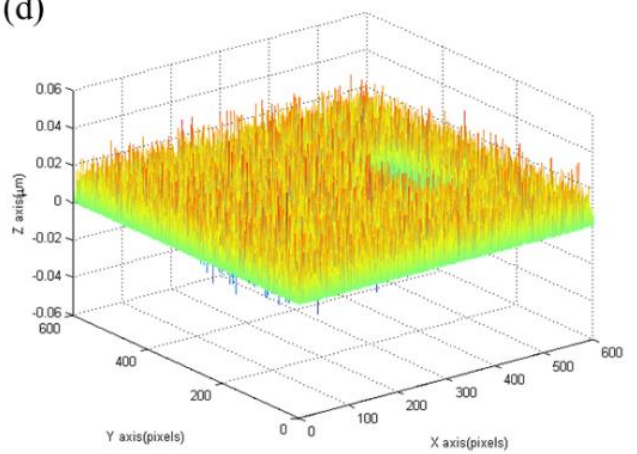

(f)

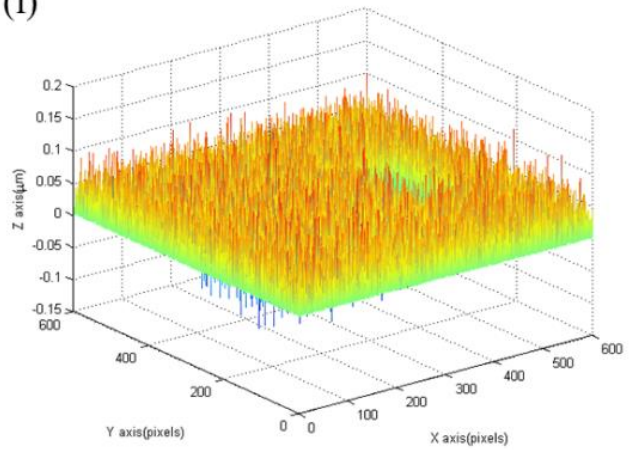

(h)

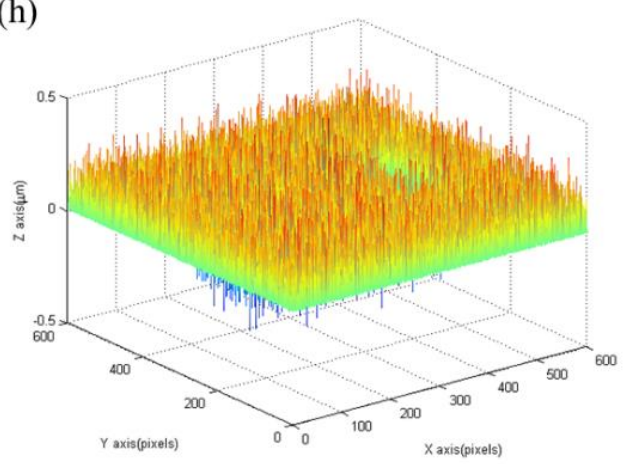

Figure 4.3 Method 2 (a) unwrapped phase when noise variance $=0$, and its (b) residue, (c) unwrapped phase when noise variance $=10^{-5}$, and its (d) residue, (e) unwrapped phase when noise variance $=10^{-4}$, and its (f) residue, (g) unwrapped phase when noise variance $=10^{-3}$, and its (h) residue. 
(a)

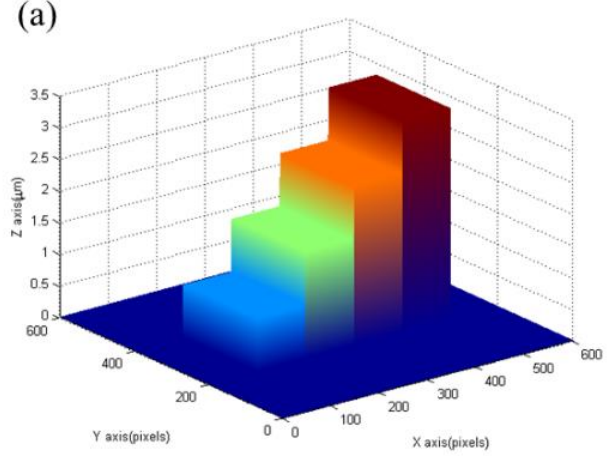

(c)

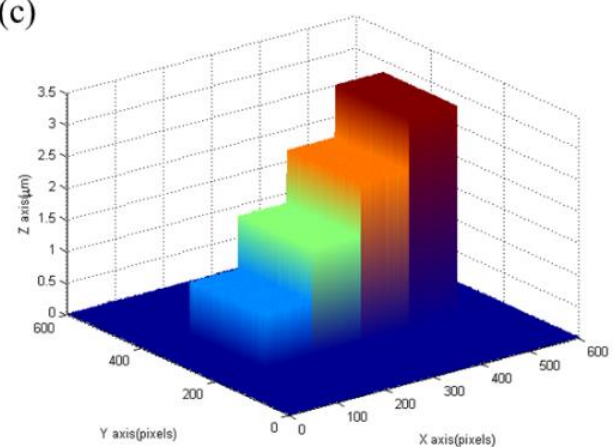

(e)

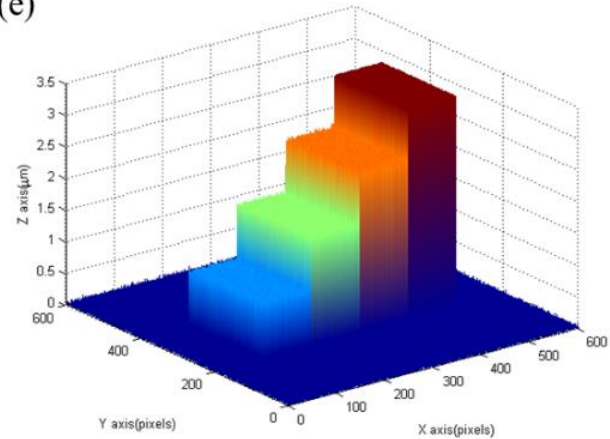

$(\mathrm{g})$

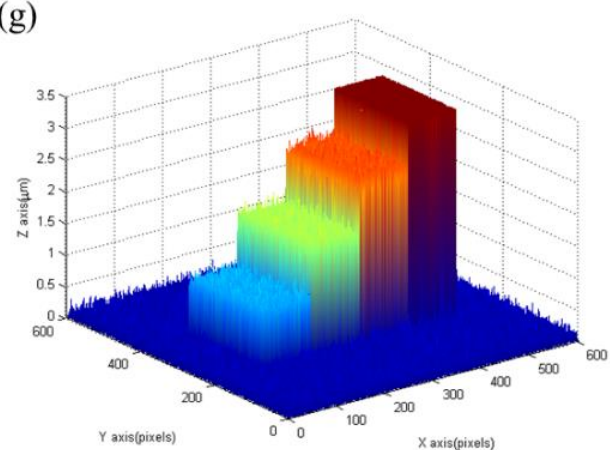

(b)

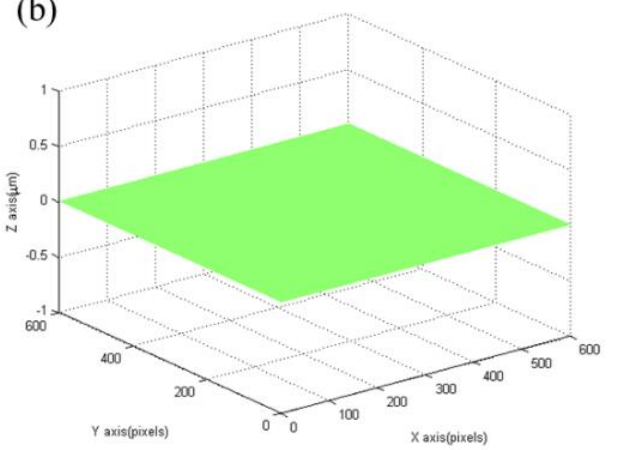

(d)

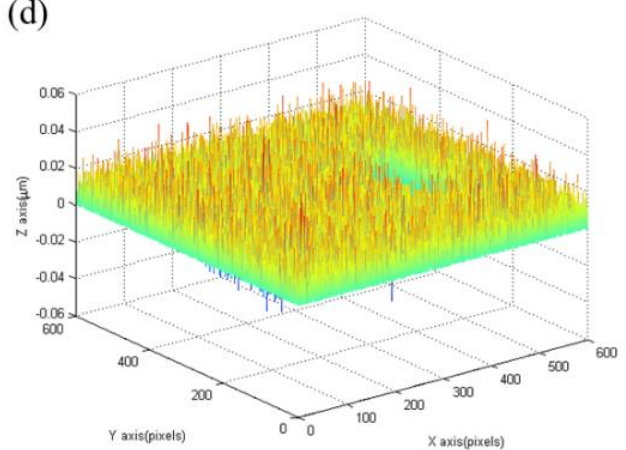

(f)

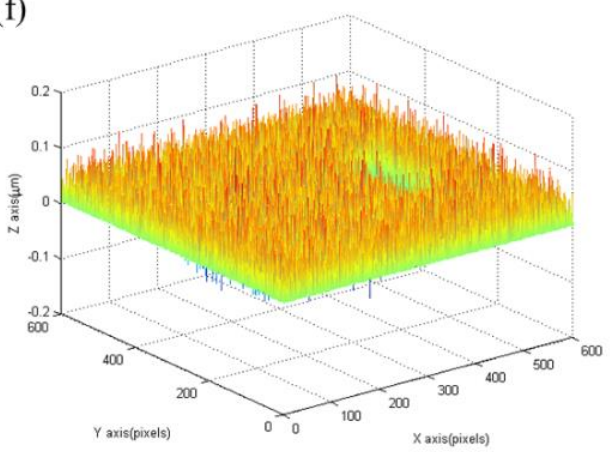

(h)

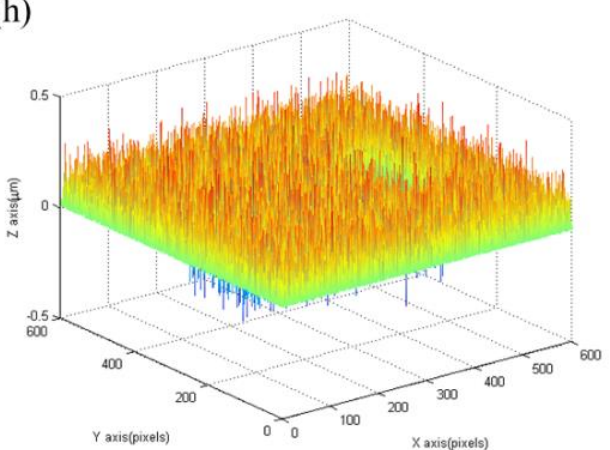

Figure 4.4 Method 3 (a) unwrapped phase when noise variance $=0$, and its (b) residue, (c) unwrapped phase when noise variance $=10^{-5}$, and its (d) residue, (e) unwrapped phase when noise variance $=10^{-4}$, and its (f) residue, $(\mathrm{g})$ unwrapped phase when noise variance $=10^{-3}$, and its (h) residue. 
(a)

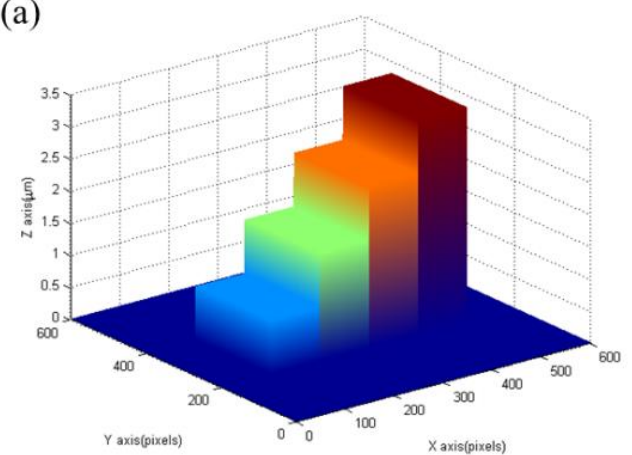

(c)

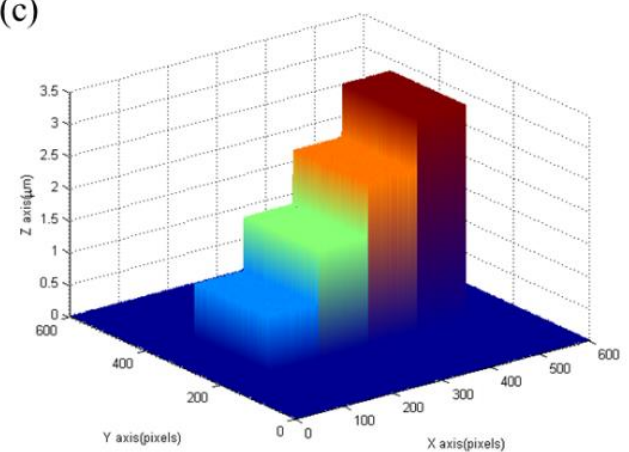

(e)

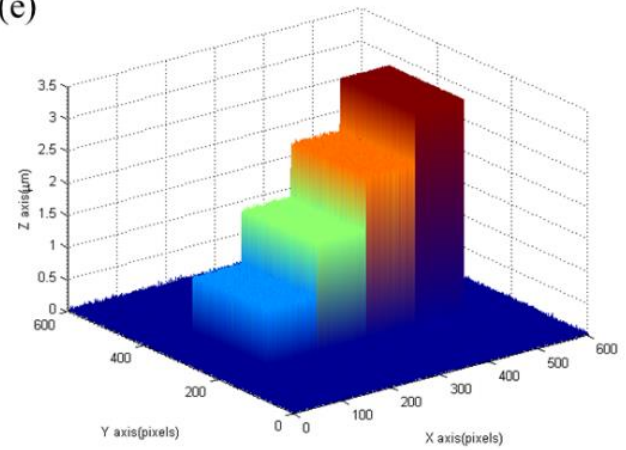

(g)

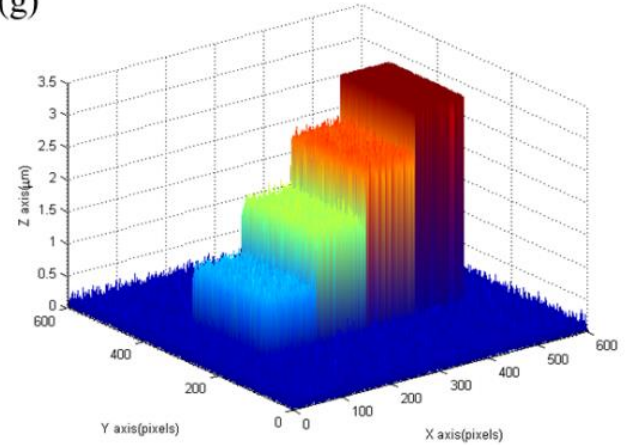

(b)

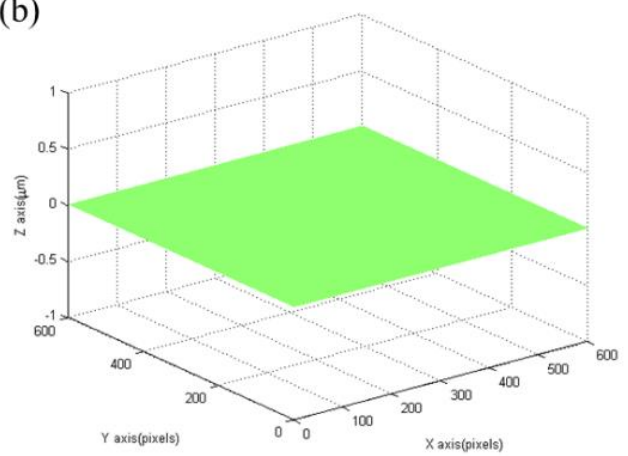

(d)

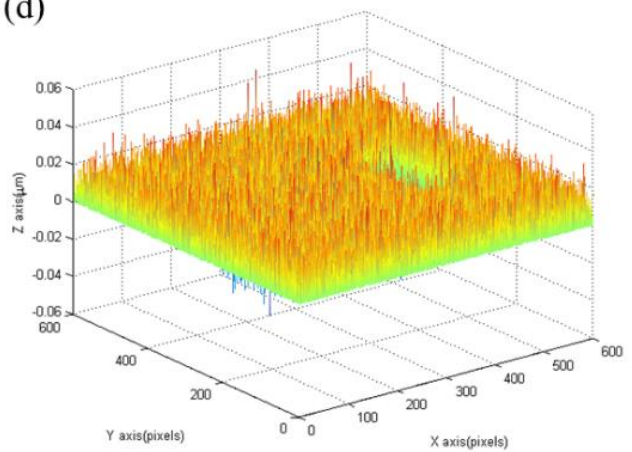

(f)

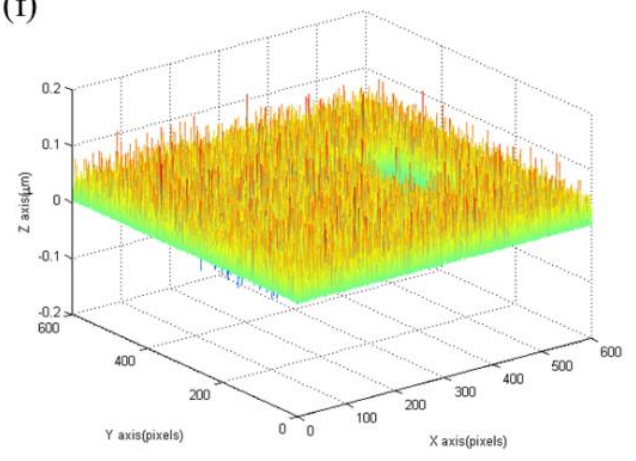

(h)

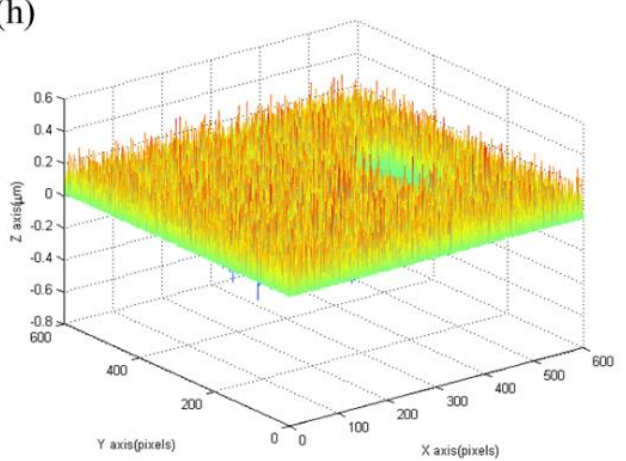

Figure 4.5 Method 4 (a) unwrapped phase when noise variance $=0$, and its (b) residue, (c) unwrapped phase when noise variance $=10^{-5}$, and its (d) residue, (e) unwrapped phase when noise variance $=10^{-4}$, and its (f) residue, (g) unwrapped phase when noise variance $=10^{-3}$, and its (h) residue. 
To compare the unwrapping goodness, red dot cross section along row $300^{\text {th }}$ in Fig. 2-5 are drawn with respect to sub number (a), (c), (e), and (g). The simulated step profile is shown in blue dash dot line. The plots regarding to method 1-4 are shown in Fig. 4.6-9, respectively. It can be seen that four methods have similar unwrapping results, and the variance in the plots go up with the noise increasing.

In order to investigate the performances the methods, Peak to Valley (PV) values, mean values and the standard deviations are then calculated in the residue. Figures 4.6-4.8 show PV curves, mean value curves and standard deviation curves, respectively. With the variance increasing, it can be seen that all these parameters also increase. These methods can unwrap correctly and draw similar residue parameters.
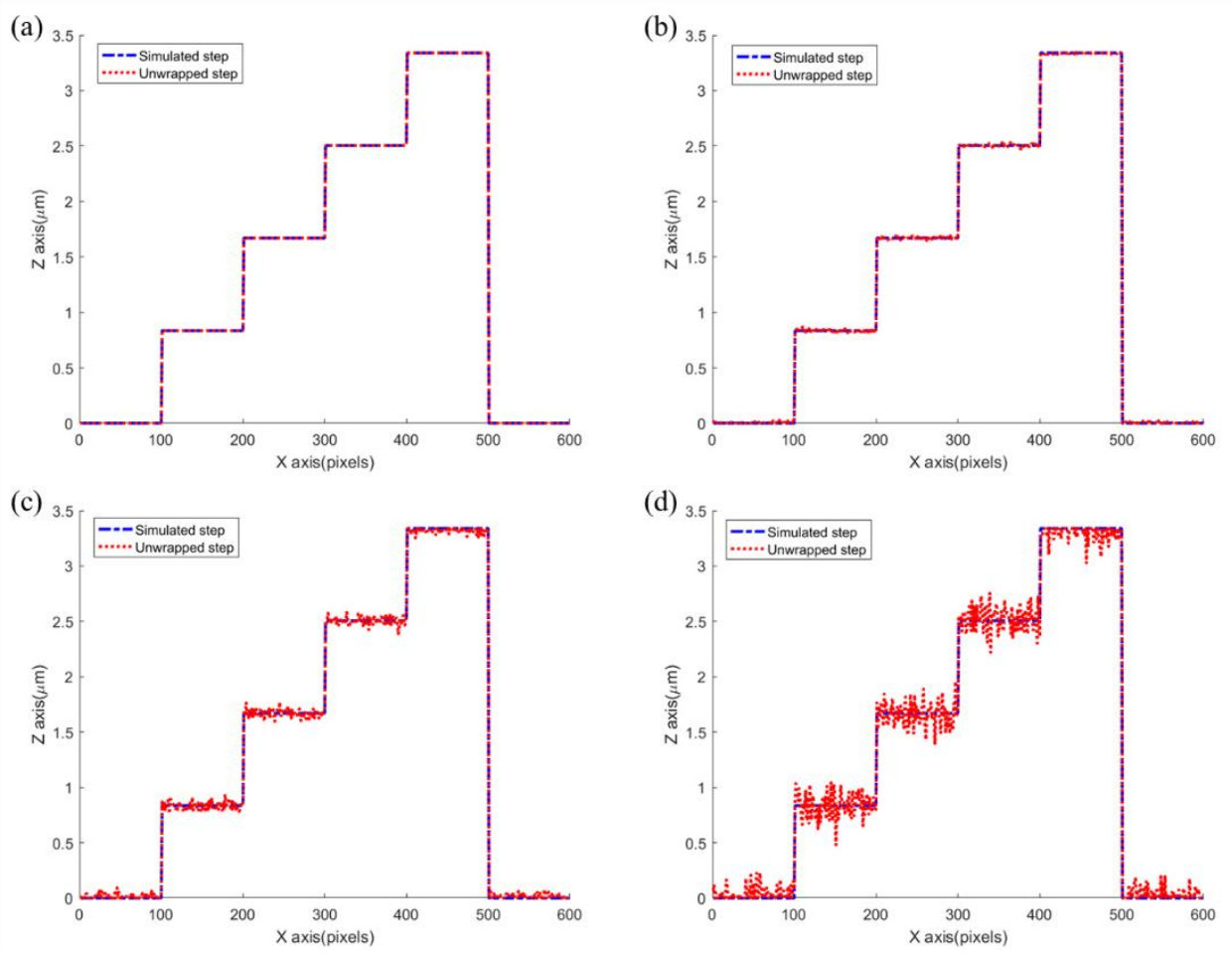

Figure 4.6 Method 1 Cross section comparison of simulated and unwrapped step profiles at line $300^{\text {th }}$, when the noise variance $=$ (a) 0 , (b) $10^{-5}$ (c) $10^{-4}$, and (d) $10^{-3}$. 

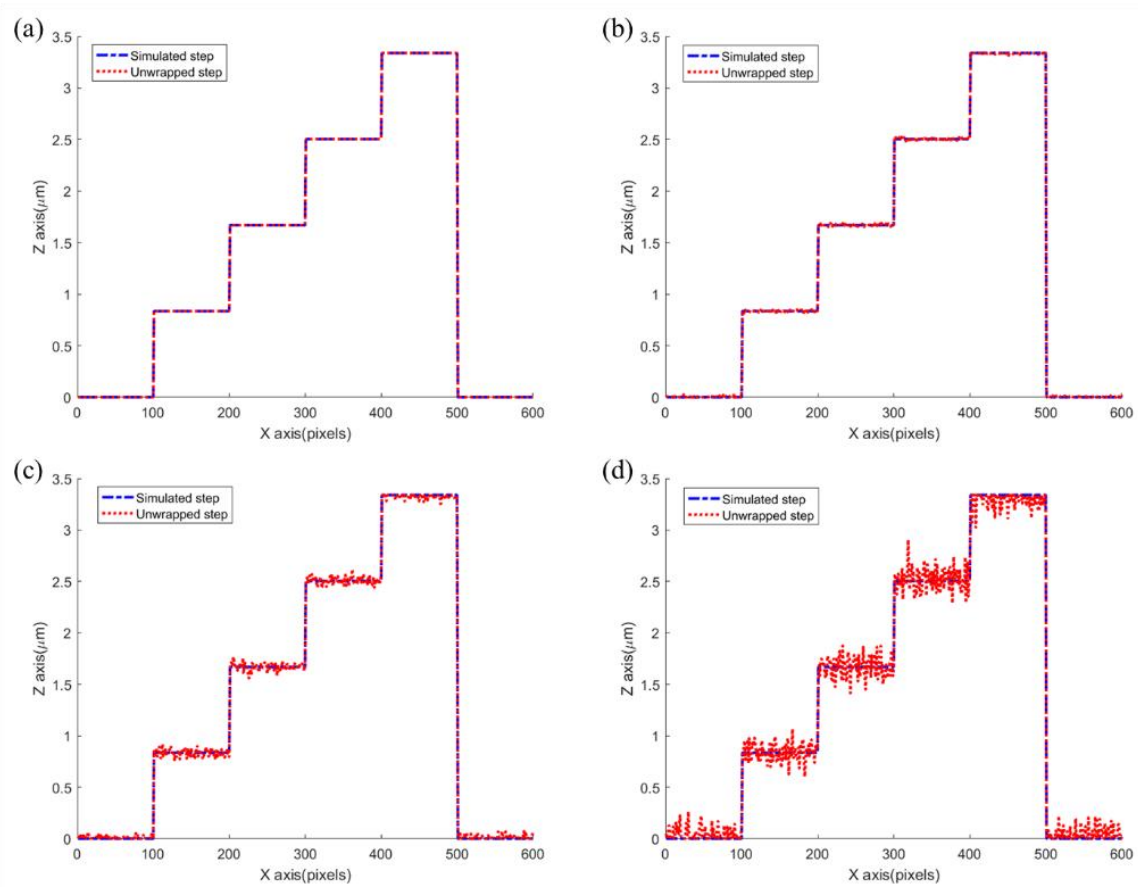

Figure 4.7 Method 2 Cross section comparison of simulated and unwrapped step profiles at line $300^{\text {th }}$, when the noise variance $=$ (a) 0 , (b) $10^{-5}$ (c) $10^{-4}$, and (d) $10^{-3}$.
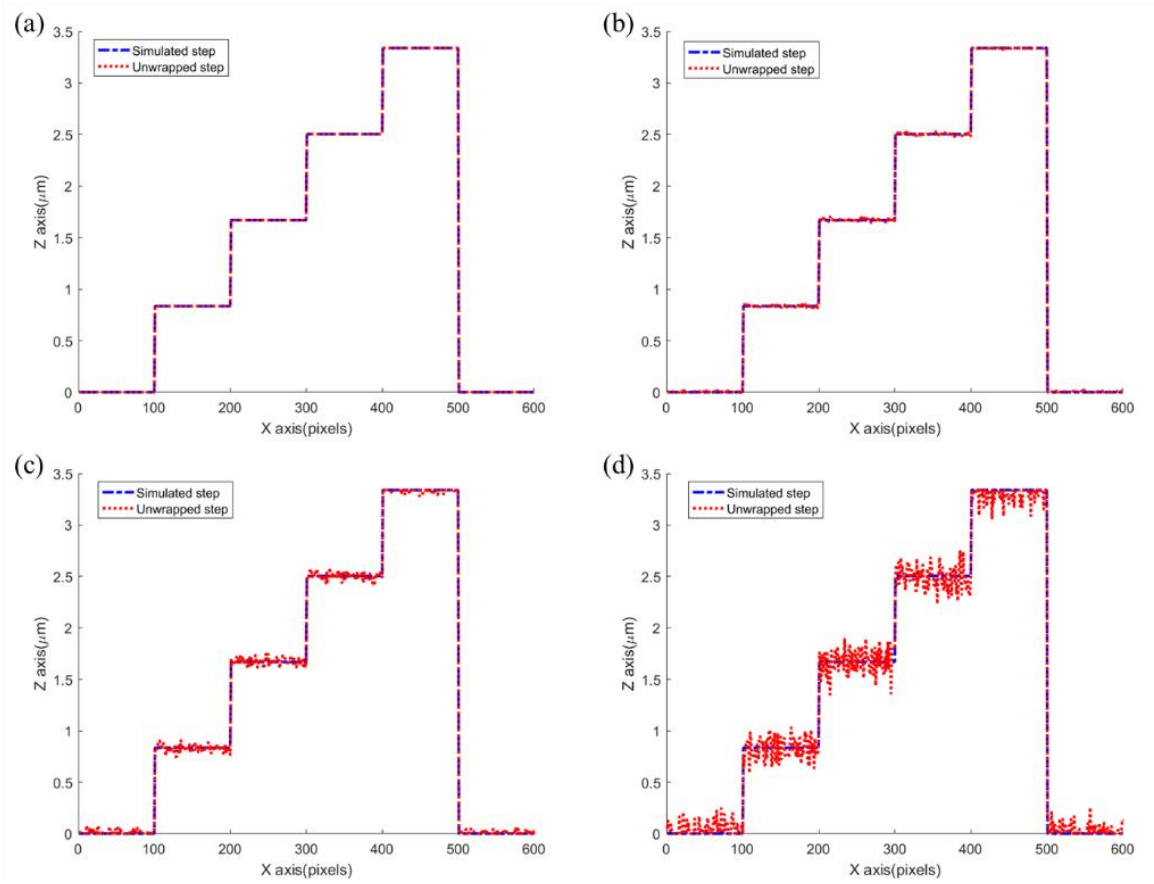

Figure 4.8 Method 3 Cross section comparison of simulated and unwrapped step profiles at line $300^{\text {th }}$, when the noise variance $=$ (a) 0 , (b) $10^{-5}$ (c) $10^{-4}$, and (d) $10^{-3}$. 

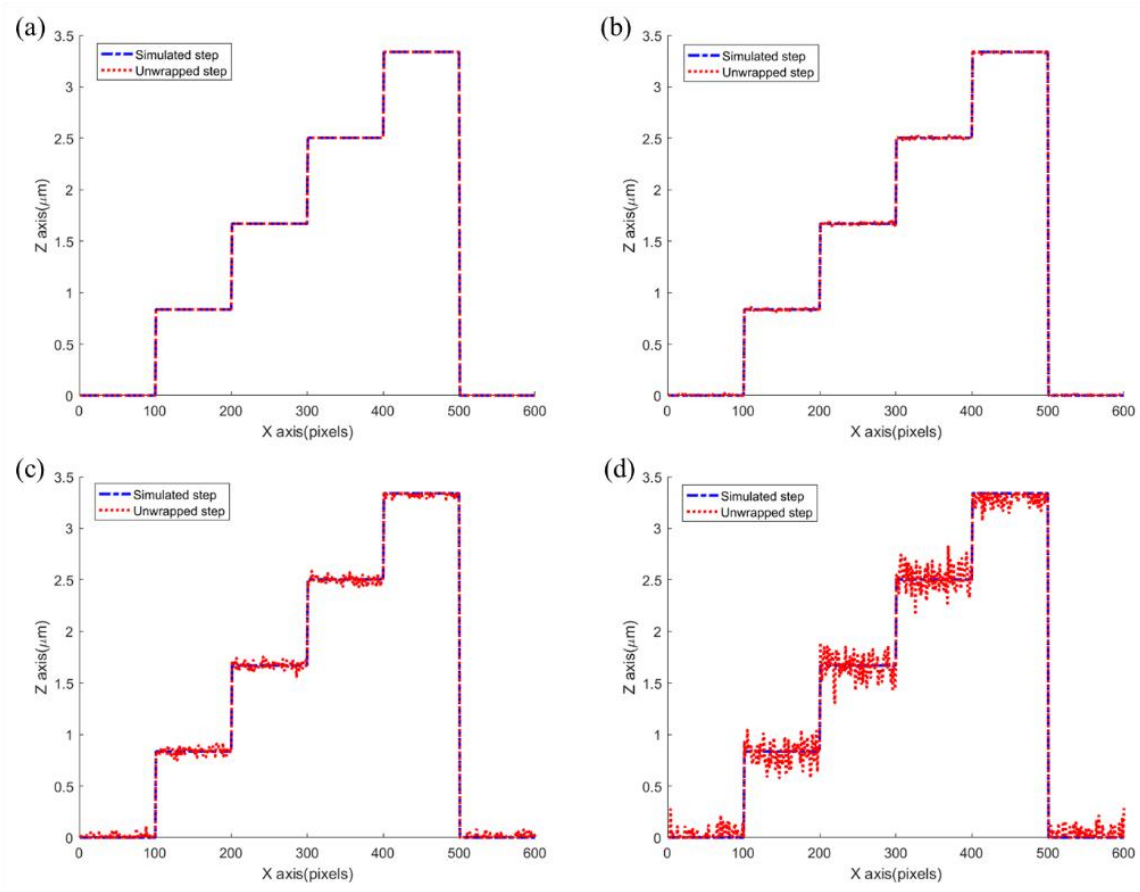

Figure 4.9 Method 4 Cross section comparison of simulated and unwrapped step profiles at line $300^{\text {th }}$, when the noise variance $=$ (a) 0 , (b) $10^{-5}$ (c) $10^{-4}$, and (d) $10^{-3}$.

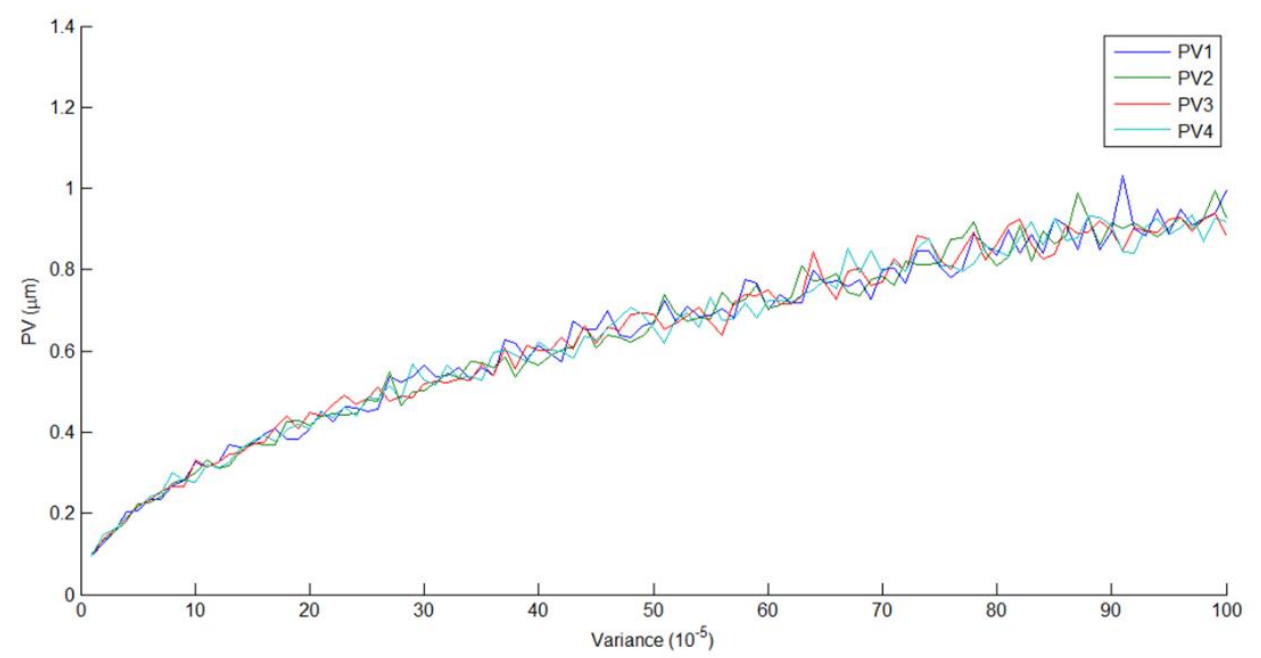

Figure 4.10 PV value curves. 


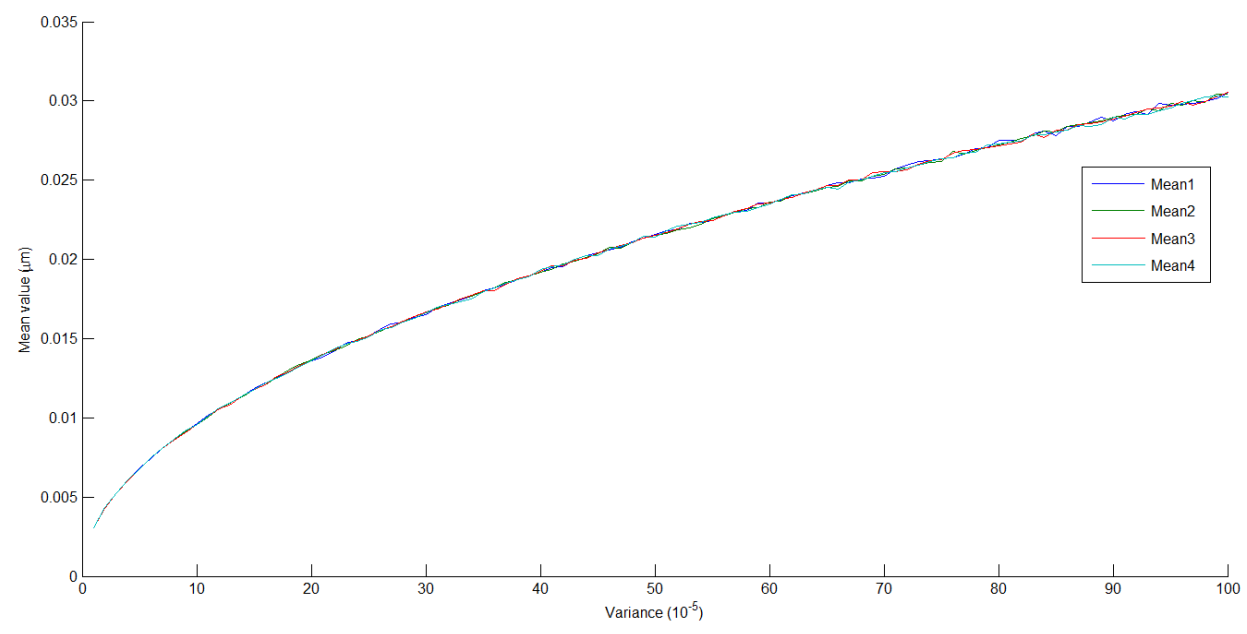

Figure 4.11 Mean value curves.

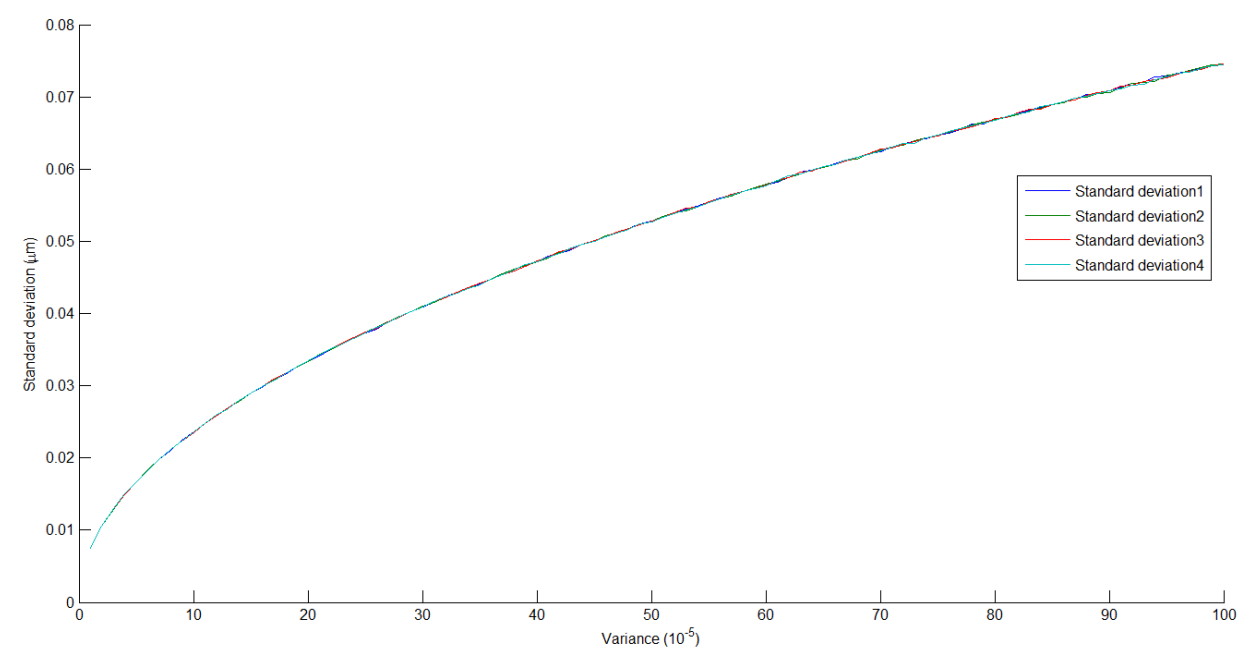

Figure 4.12 Standard deviation curves

\subsection{Experiment and results}

\subsubsection{Testing of MEMS sample}

The schematic of the experimental arrangement is shown in Fig. 4.13. A lensless Michelson interferometer is employed for measurement. Light from the laser is split by a BS into two beams: one beam goes to the sample and reflects back, which forms the object beam; the other beam is reflected by the mirror and serves as the reference beam. 
The two beams then meet and interfere in front of CCD camera. The fringe patterns are then recorded by CCD camera as the digital hologram for numerical reconstruction. In the experiment, the distance from the light source to the mirror is $35 \mathrm{~mm}$. The $\mathrm{BS}$ is a $25.4 \mathrm{~mm}$ cube. The distance of the CCD camera sensor to the BS is $2 \mathrm{~mm}$. The reason why the CCD camera can be so close to the BS is because it is a board camera. The working distance for the system is $8 \mathrm{~mm}$.

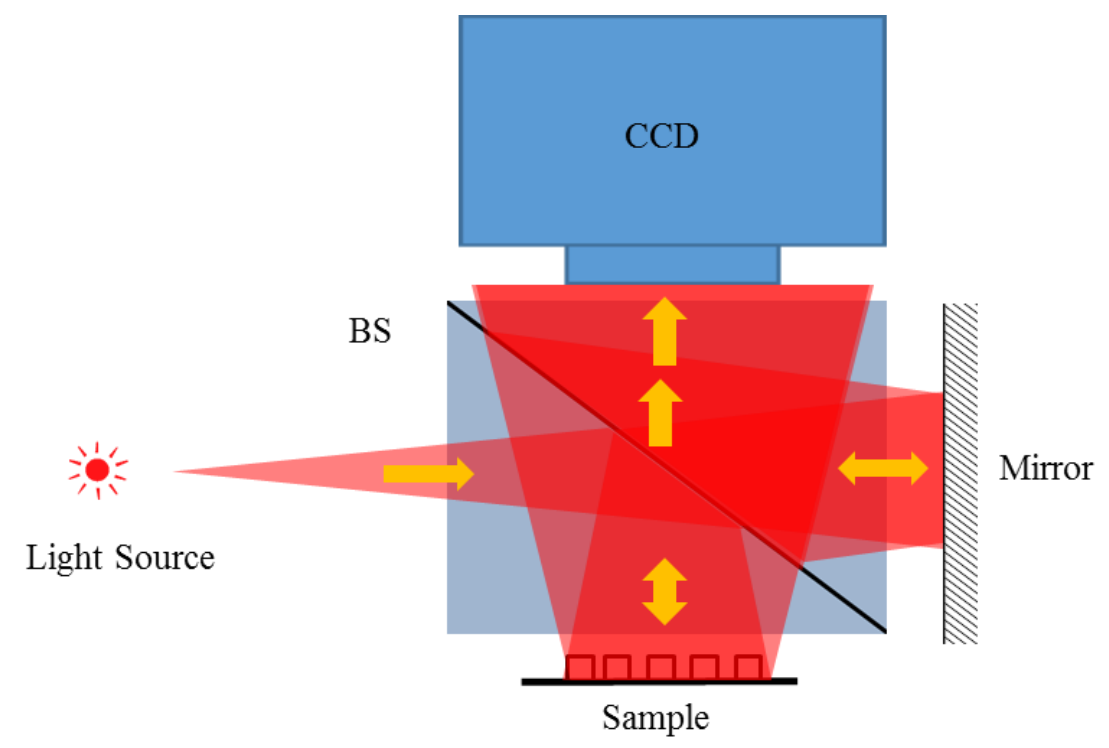

Figure 4.13 Schematic of Michelson interferometer for the MEMS sample measurement.

A MEMS sample with bump was first tested by above digital holographic system [2, 123]. The light was switched between two laser systems $(632.8 \mathrm{~nm}$ and $656.6 \mathrm{~nm})$. The CCD camera used in the experiment was $1024 \times 768$ pixels along with pixel size $4.65 \mu \mathrm{m} \times 4.65 \mu \mathrm{m}$. Two digital holograms were captured and recorded separately as in Fig. 4.14. The numerical reconstruction was performed by commercial software supported by D’Optron Pte Ltd. The reconstructed wrapped phases were shown in Fig. 4.15. Because we use exactly the same configuration in digital hologram acquisition, there is no 
curvature mismatch problem which often needs extra compensation in many dualwavelength structures.

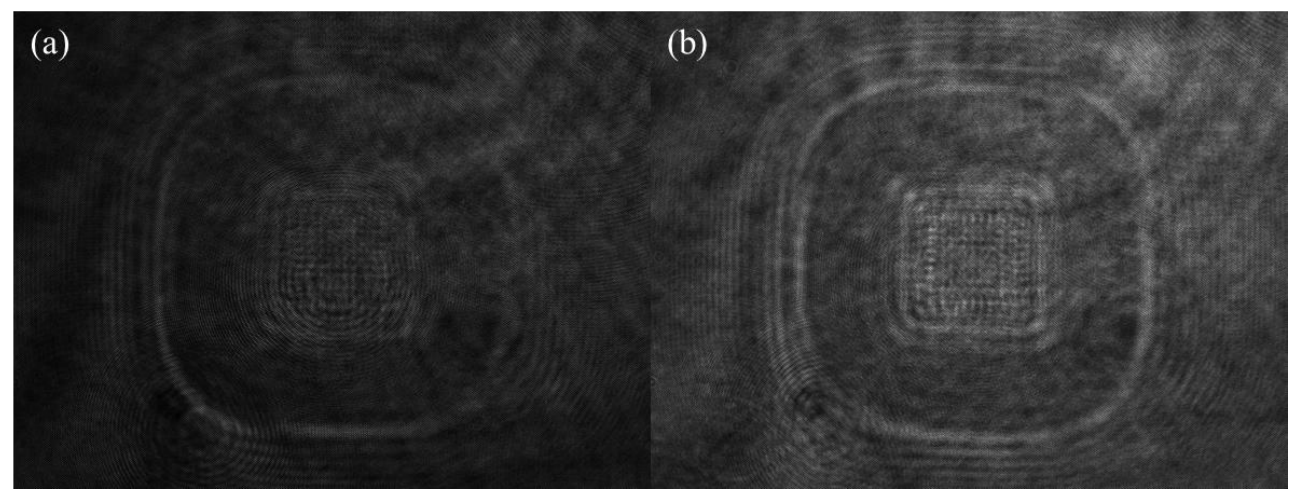

Figure 4.14 Digital hologram of the MEMS sample at the wavelength of (a) $632.8 \mathrm{~nm}$ and (b) $656.6 \mathrm{~nm}$.

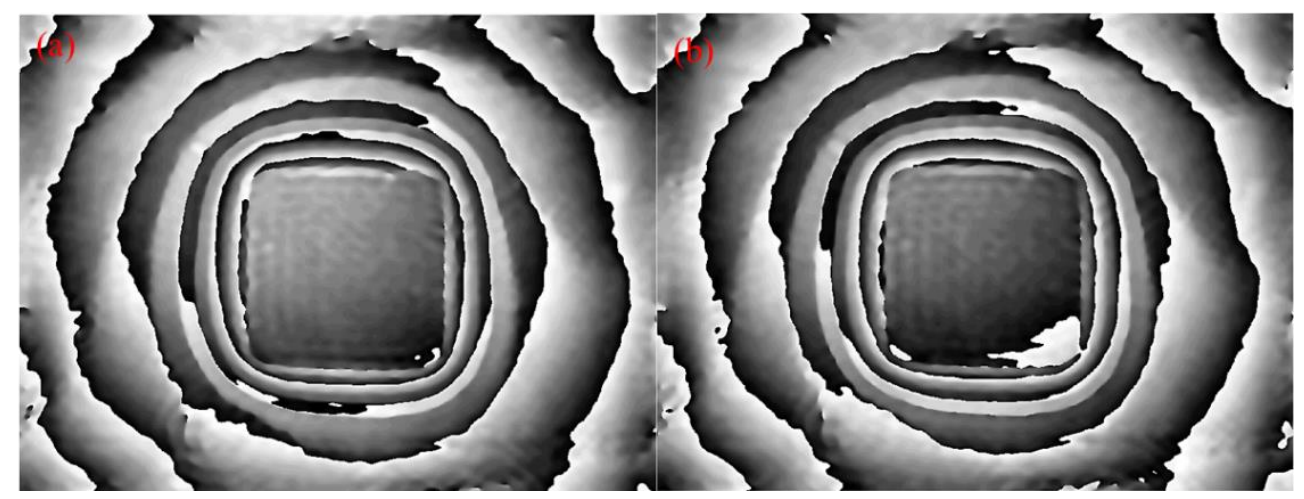

Figure 4.15 Wrapped phase of the MEMS sample for the wavelength of (a) $632.8 \mathrm{~nm}$ and (b) $656.6 \mathrm{~nm}$.

To experimentally demonstrate the performance of the linear programming method, a point located at $(512,384)$ is selected for generating the search process. For boundary conditions, the integers corresponding to two wavelengths are 23 and 24, respectively. The value map with respect to Eq. 10 is shown in Fig. 4.16. The shape of the map is like a slope valley. The minimum value we are looking for exists along the valley bottom. Through search, the minimum point is highlighted by a red point. In this example, it is noted that although the optimal match for two wavelengths appear in upper of the diagonal, the difference for the values along the diagonal is quite small actually. 
Therefore, the boundary condition set up is very important. Too large boundary will lead search time increase rapidly. While too small boundary may miss the best match point.

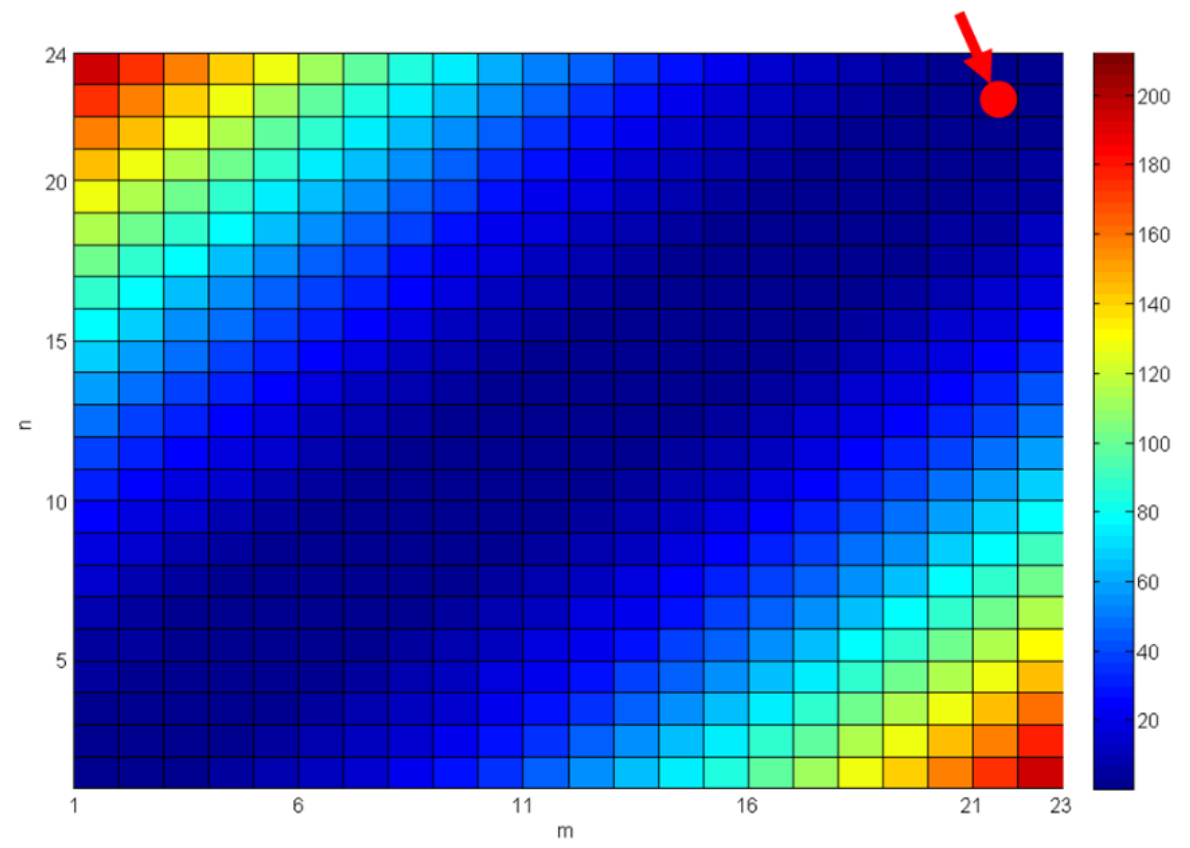

Figure 4.16 The map for the square of the height difference.

Figures 17(a) and (b) are the unwrapped phase and its 3D shape with the proposed method. It is clear that the object is about $14 \mu \mathrm{m}$ high which coincides with the height value calibrated by confocal microscope. This height is totally beyond single wavelength resolvable capability. While in DWDH, it is possible to recover the true shape.

(a)

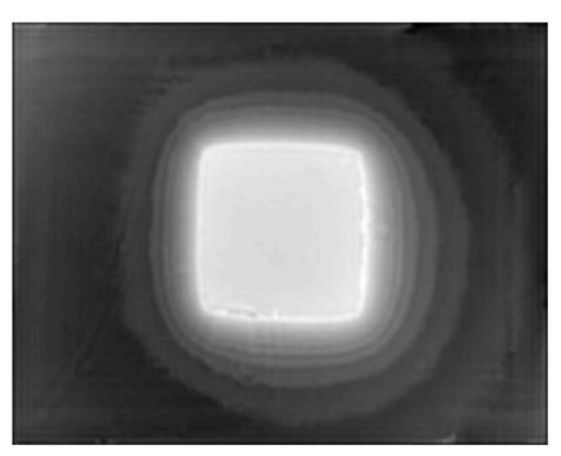

\section{(b)}

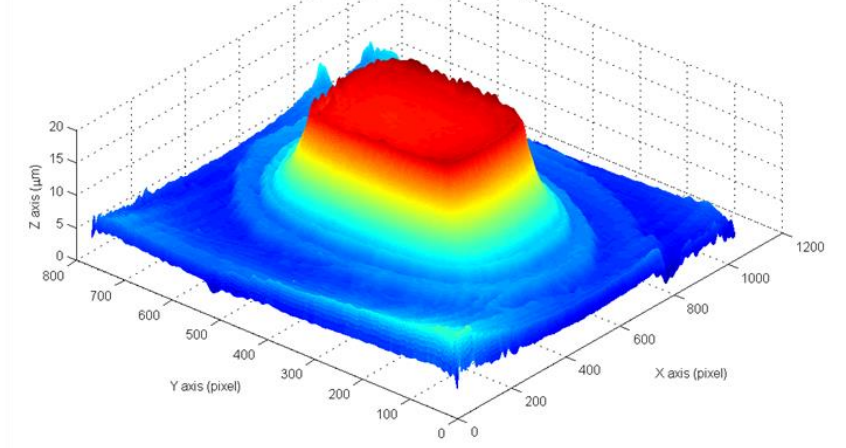

Figure 4.17 (a) Unwrapped phase of the MEMS sample and (b) its height map with 3D rendering. 


\subsubsection{Testing of cylindrical lens}

The schematic of the experimental arrangement is shown in Fig. 4.18. A modified Tyman-Green interferometer is employed for measurement. Light from the laser source is coupled and split into two beams. One collimated beam goes through the cylinder lens which forms the object beam; the other collimated beam serves as the reference beam. The two beams then meet and interfere at BS with a small angular offset between them. The interference pattern between the two beams is captured and recorded by CCD camera. In the experiment, the distance from the cylindrical lens to the CCD camera is $357 \mathrm{~mm}$.

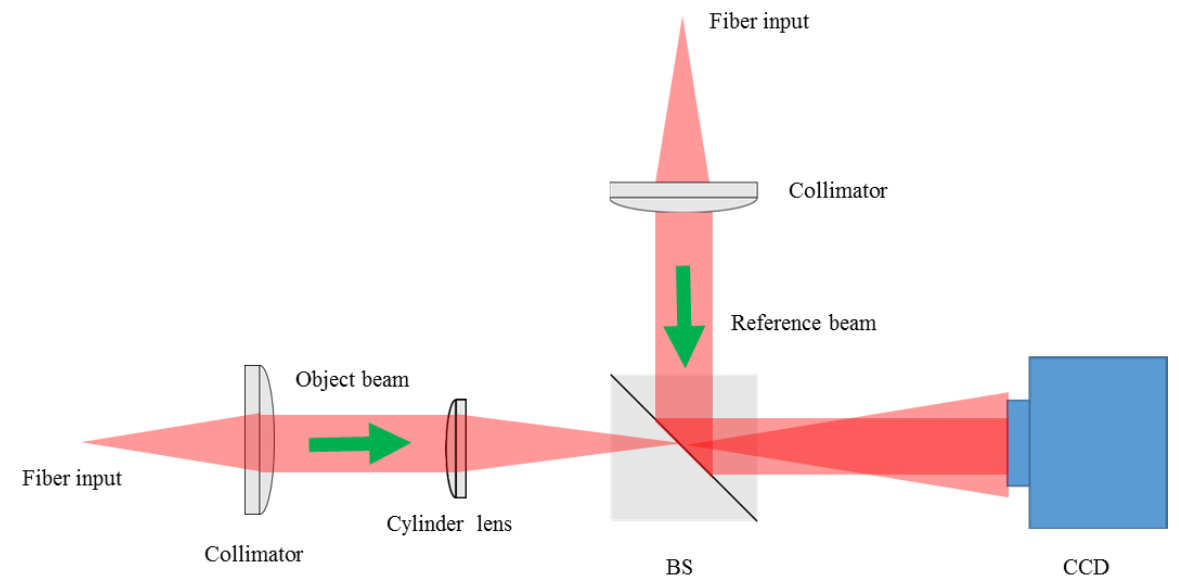

Figure 4.18 Schematic of transmissive interferometer for cylindrical lens measurement.

The cylindrical lens is from Newport (model: CKX006). It is an uncoated N-BK7 planoconvex cylindrical lens. Its effective focal length is $6.4 \mathrm{~mm}$. It is designed and applied for laser electro-optic. The light was again switched between two laser systems (632.8and $656.6 \mathrm{~nm})$. The CCD camera used in experiment was $1280 \times 960$ pixels along with pixel size $4.65 \mu \mathrm{m} \times 4.65 \mu \mathrm{m}$. By double-exposure, two digital holograms are recorded sequentially. Numerical reconstructions are then performed. Two wrapped phases are 
obtained and shown in Fig. 20(a) and (b). It can be seen that two wrapped phases are similar visually.

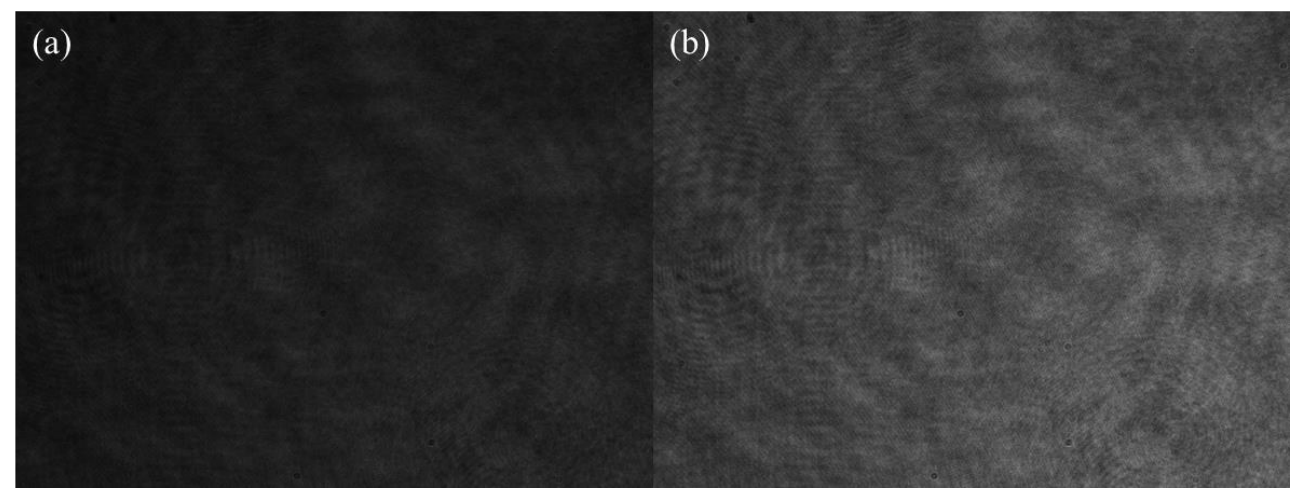

Figure 4.19 Digital hologram for the cylindrical lens at the wavelength of (a) $632.8 \mathrm{~nm}$ and (b) $656.6 \mathrm{~nm}$.

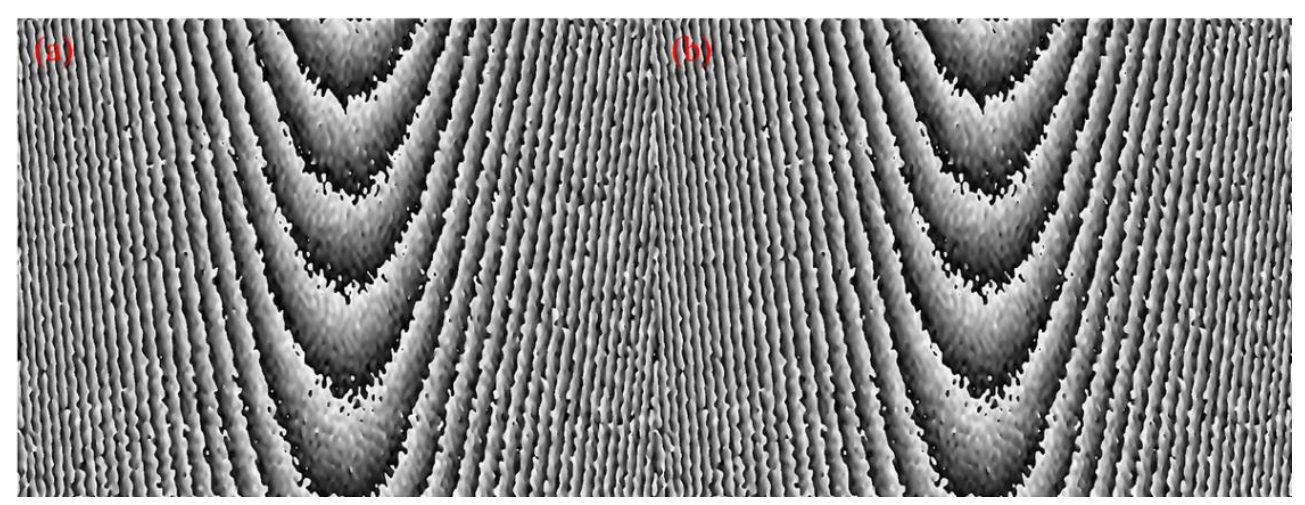

Figure 4.20 Wrapped phase of the cylindrical lens with wavelength of (a) $632.8 \mathrm{~nm}$ and (b) $656.6 \mathrm{~nm}$.

(a)

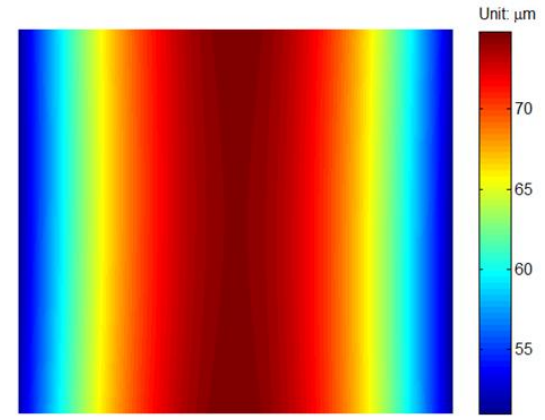

(b)

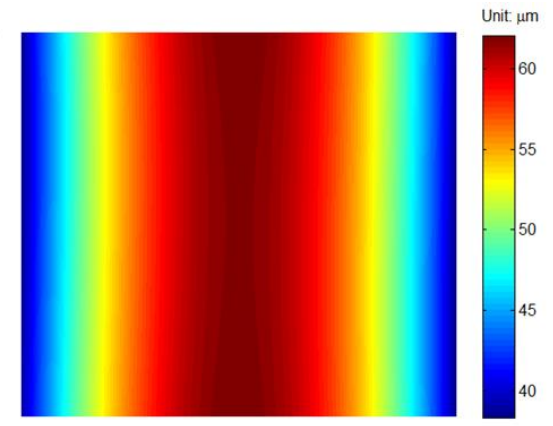

Figure 4.21 Reconstructed height map with Goldstein algorithm for the wavelength of (a) $632.8 \mathrm{~nm}$ and (b) $656.6 \mathrm{~nm}$.

In order to demonstrate the difference of the dual-wavelength unwrapping and common phase unwrapping, we unwrapped two phases in Fig. 4.21. The phase unwrapping 
algorithm is Goldstein algorithm [124]. In Fig. 4.21, the unwrapped phases are shown. It can be seen that the peak to valley values for both phases are proximately $25 \mu \mathrm{m}$. However, this value is far beyond the true value.

Again, we apply the proposed algorithm to unwrap the phase. The unwrapped phase and its 3D rendering are displayed in Fig. 4.22(a) and (b). It should be noted that the peak to valley value is proximately $60 \mu \mathrm{m}$ which is much different from above $25 \mu \mathrm{m}$ and close to true value. To compare in detail, a plot along $480^{\text {th }}$ row is drawn in Fig. 4.22(c). The ideal profile is also plotted in the same graph in red dash line. We can see that two curves are in good agreement.

(a)

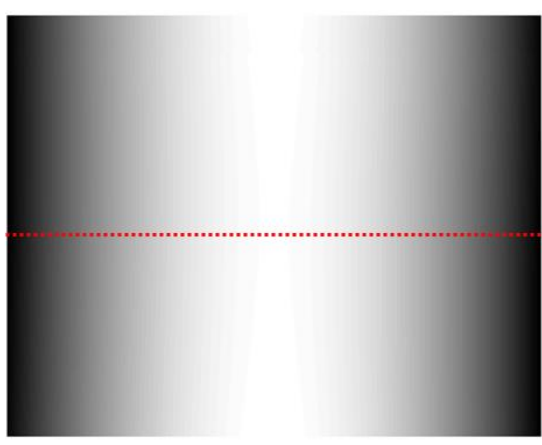

(b)

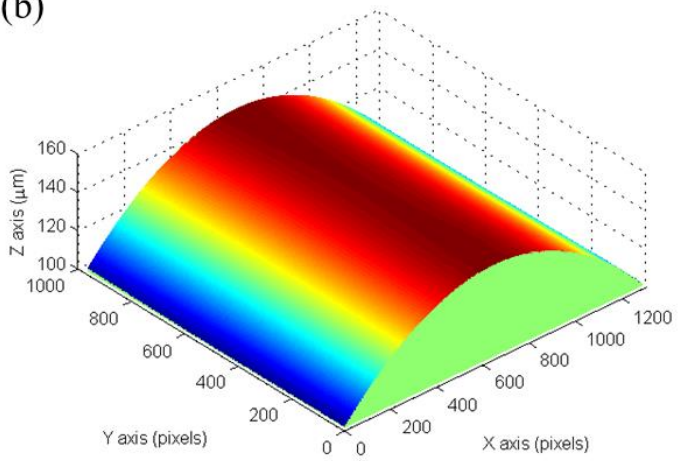

(c)

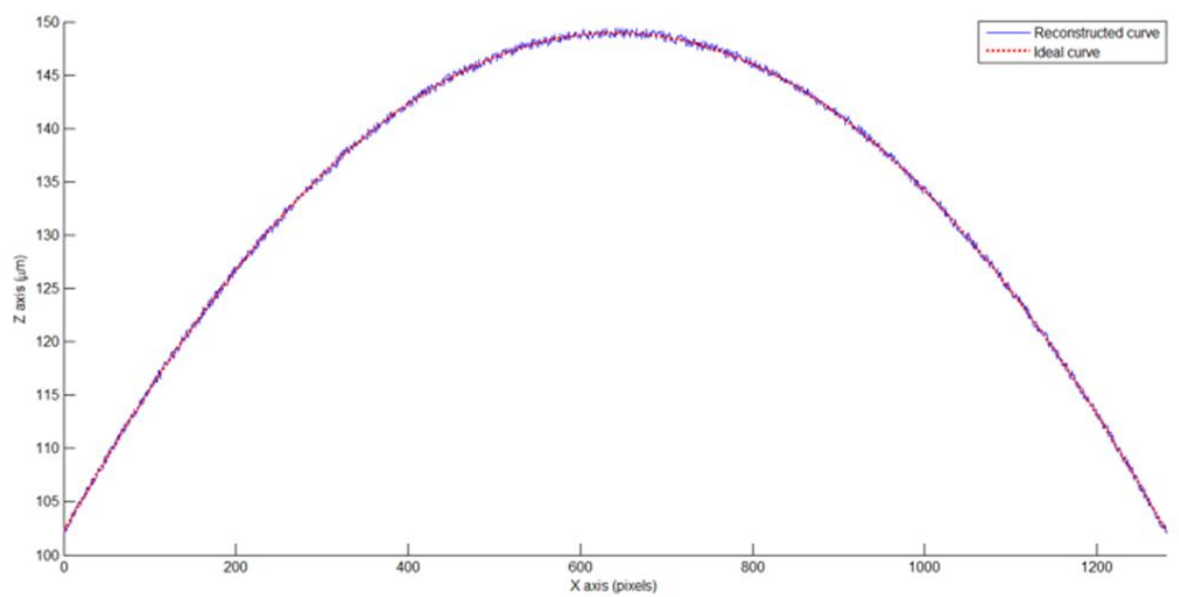

Figure 4.22 (a) Unwrapped phase of the cylindrical lens, (b) 3D rendering of the height map, (c) curves comparison. 


\subsubsection{Testing of aspheric lens}

The schematic of the experimental arrangement is shown in Fig. 4.23. A modified Tyman-Green interferometer is employed for measurement. Light from the laser source is coupled and split into two beams. One collimated beam goes through the cylinder lens which forms the object beam; the other collimated beam serves as the reference beam. The two beams then meet and interfere at BS with a small angular offset between them. The interference pattern between the two beams is captured and recorded by CCD camera. In the experiment, the distances from the aspheric lens to the triplet and the triplet to the CCD camera are $70 \mathrm{~mm}$ and $480 \mathrm{~mm}$, respectively.

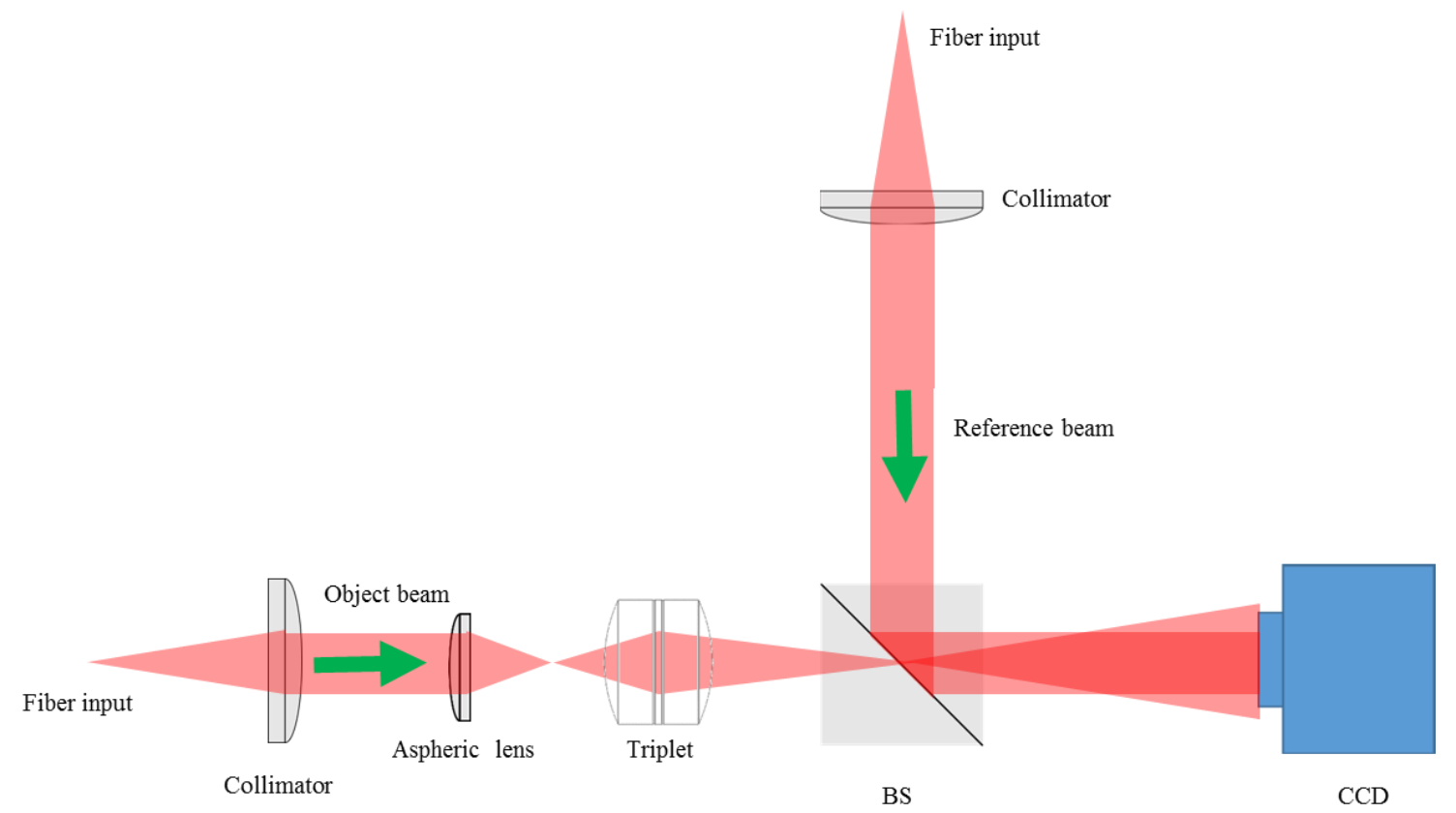

Figure 4.23 Schematic of transmissive interferometer for aspheric lens measurement.

The aspheric lens is from Newport (model: KPA16) with $25.00 \mathrm{~mm}$ diameter and $37.50 \mathrm{~mm}$ focal length, respectively. The light was again switched between two laser systems (632.8and $656.6 \mathrm{~nm}$ ). The CCD camera used in experiment was $1280 \times 960$ pixels along with pixel size $4.65 \mu \mathrm{m} \times 4.65 \mu \mathrm{m}$. By double-exposure, two digital holograms are 
recorded sequentially as shown in Fig. 4.24. Numerical reconstructions are then performed on two digital holograms. Two wrapped phases are obtained and shown in Fig. 4.25(a) and (b). It can be seen that two wrapped phases are modulated by different fringe patterns at the same position, but they have similar fringe number in total.

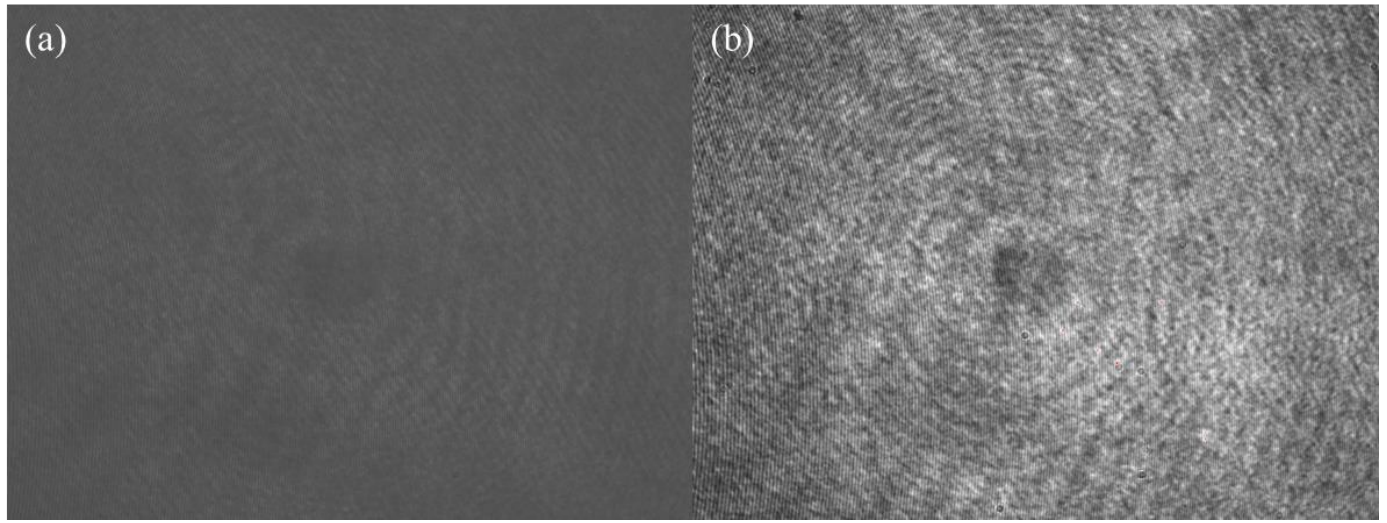

Figure 4.24 Digital hologram for the aspheric lens at the wavelength of (a) $632.8 \mathrm{~nm}$ and (b) $656.6 \mathrm{~nm}$.

(a)

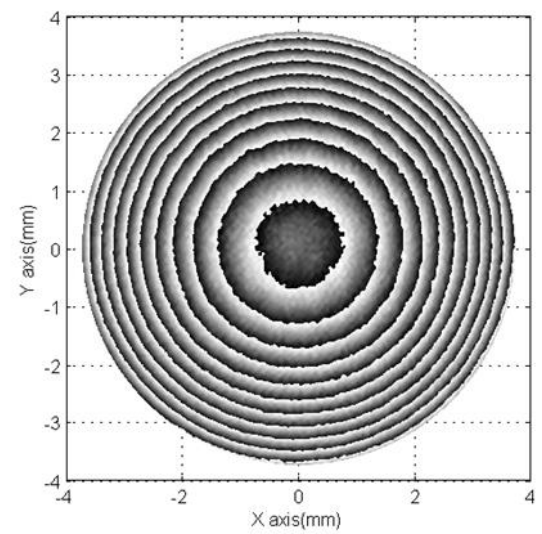

(b)

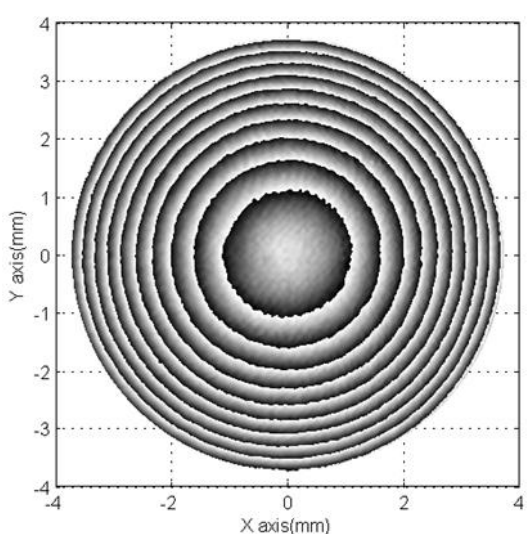

Figure 4.25 Wrapped phase of the aspheric lens with wavelength of (a) $632.8 \mathrm{~nm}$ and (b) $656.6 \mathrm{~nm}$.

In order to demonstrate the difference of the dual-wavelength unwrapping and common phase unwrapping, we unwrapped two phases in Fig. 26. The phase unwrapping algorithm is Goldstein algorithm [124]. It can be seen that the peak to valley values for both phases are proximately $65 \mu \mathrm{m}$. However, this value is far beyond the true value. 
(a)

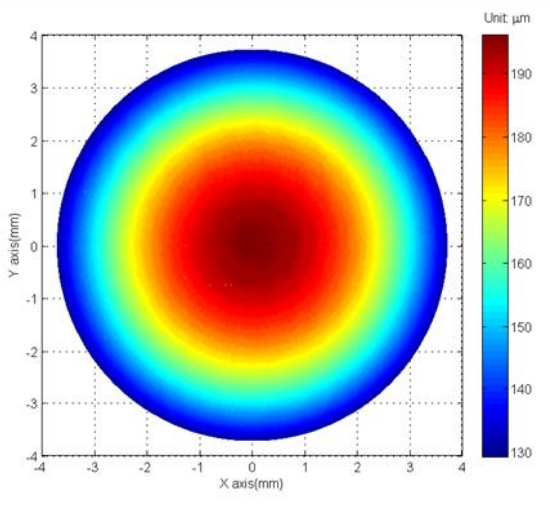

(b)

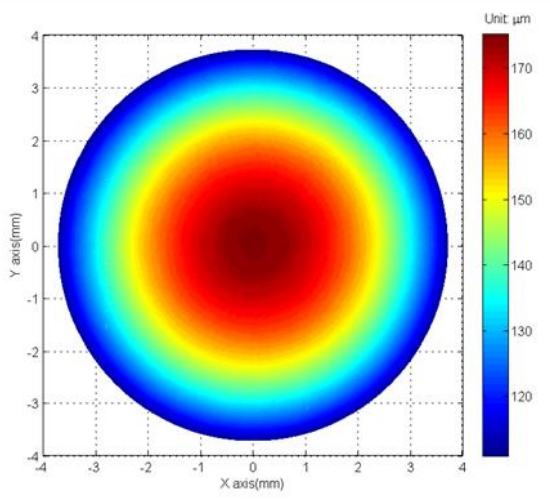

Figure 4.26 Reconstructed height map with Goldstein algorithm for the wavelength of (a) $632.8 \mathrm{~nm}$ and (b) $656.6 \mathrm{~nm}$.

Again, we apply the proposed algorithm to unwrap the phase. The unwrapped phase and its 3D rendering are displayed in Fig. 4.27 (a) and (b). It should be noted that the PV value is proximately $325 \mu \mathrm{m}$ which is much different from above $65 \mu \mathrm{m}$ and coincides with true value. To compare in detail, a plot along $480^{\text {th }}$ row is drawn in Fig. 4.27(c). The ideal profile is also plotted in the same graph in red dash line. We can see that two curves are in good agreement. Due to the noise existing in the system, the measured curve is affected and fluctuates in details. 
(a)

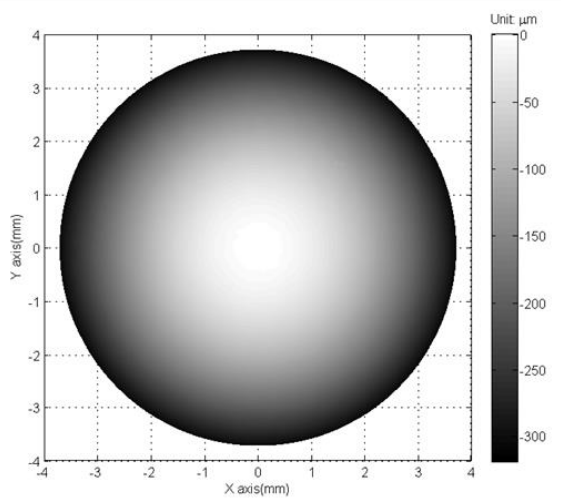

(b)

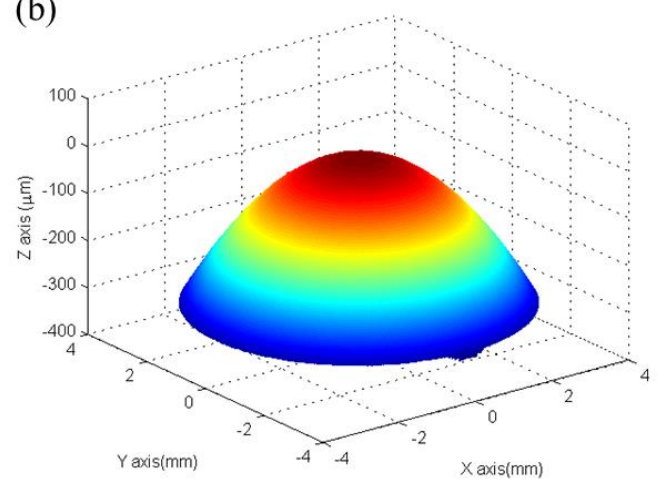

(c)

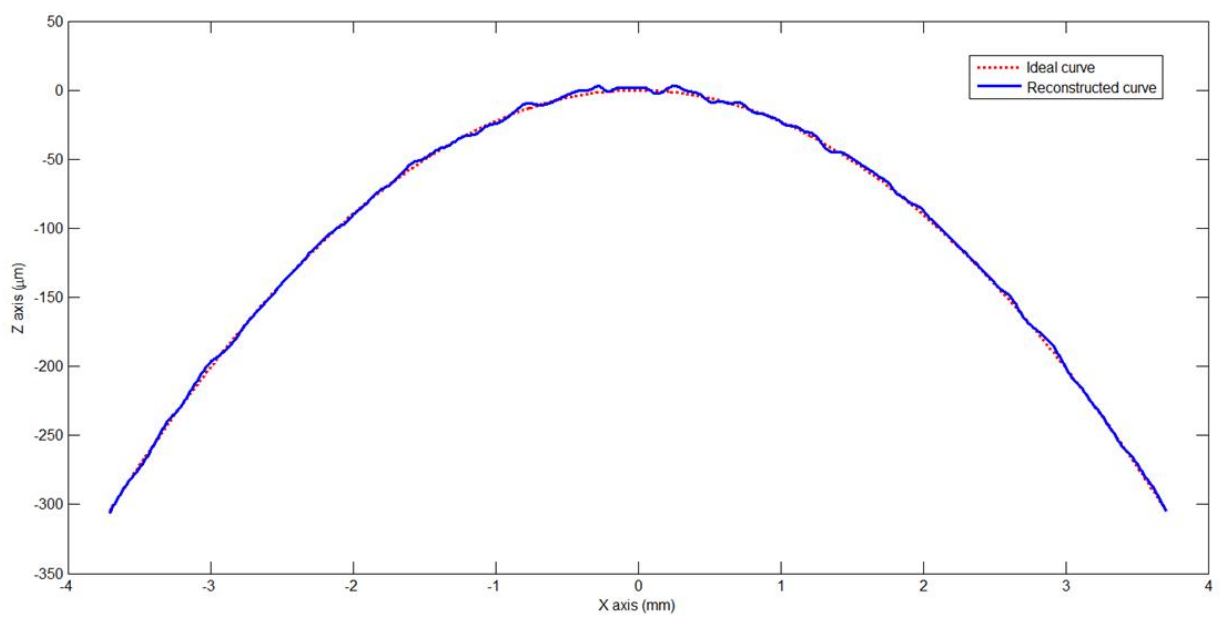

Figure 4.27 (a) Unwrapped phase of the aspheric lens, (b) 3D rendering of the height map, (c) curves comparison.

\subsection{Chapter summary}

In summary, the axial resolution of digital holographic system is analyzed. Three modified and a linear programming dual-wavelength phase unwrapping have been studied. By giving convergence standard as well as the boundary conditions, the four methods have well solved the problem in searching optimal integer pair. Moreover, owning to its convergence is depending on the variance of height, the four methods are robust in presence of noise. In experiments, a MEMS sample, a cylindrical lens, and an aspheric lens are tested, and the results show DWDH is able to recover the discontinuities that exceeds the resolution limitation in single wavelength digital holography. Of course, 
the dual-wavelength phase unwrapping methods do not limit to digital holography but also can be applied in other interferometric imaging techniques. 


\section{SYNTHETIC APERTURE DIGITAL HOLOGRAPHY TO MEASURE ASPHERIC SURFACE}

In this chapter, the lateral resolution improvement of the synthetic aperture digital holography is first analyzed. As a result, synthetic aperture digital holography can achieve high axial resolution and large field of view at the same time.

Next, the first order spectrum based stitching method is proposed. The composition depends on high resolution motorized stages, and the composition size is predefined first before the scanning starts. In scanning, FFT is performed on sub digital holograms, and the first order spectrum is filtered and accumulated. Only one-time inverse FFT is required after scanning is finished. Thus the final result is a wrapped phase image. This method changes image melting to phase unwrapping which is a mature tool in implementation. In the meanwhile, it also avoids the uncertainty in stitching the digital holograms.

In the experiment, measurement of a high-precision aspheric lens is done, and the result indicates the proposed method is straightforward and highly implementable.

\subsection{Lateral resolution improvement of synthetic aperture digital holography}

In digital holography, the lateral resolution is limited by the FOV and the number of pixels in the detector array. MO can be introduced to improve the lateral resolution, but FOV will decrease due to the magnification. The lateral resolution and FOV must be sacrificed to achieve the other. However, both high lateral resolution and large FOV are 
always desired. Aperture synthesis is a post-process technique which is able to achieve both high lateral resolution and a wide field-of-view simultaneously. Furthermore, aperture synthesis can improve the lateral resolution according to the lateral resolution formula in digital holography

$$
\operatorname{Res}=\frac{1}{M} \frac{\lambda d}{2 D}
$$

where Res is the lateral resolution, $M$ is magnification, $d$ is the imaging distance and $D$ is half width of CCD. From Eq. 5.1, we know that after stitching the holograms recorded from synthetic aperture, the new single hologram will has a new half width $D^{\prime}$ which is larger than $D$.As a result, the lateral resolution Res is improved when the denominator $D$ turns larger to $D^{\prime}$.

To demonstrate analysis, four segmented holograms of a USAF target in the left side of Fig. 5.1 are displayed in clockwise. The image in the right side of Fig. 5.1 is the synthetic hologram. The segmented holograms are exactly $1 / 2$ of the synthetic hologram in size. The overlaps in horizontal and vertical directions are all set to zero.
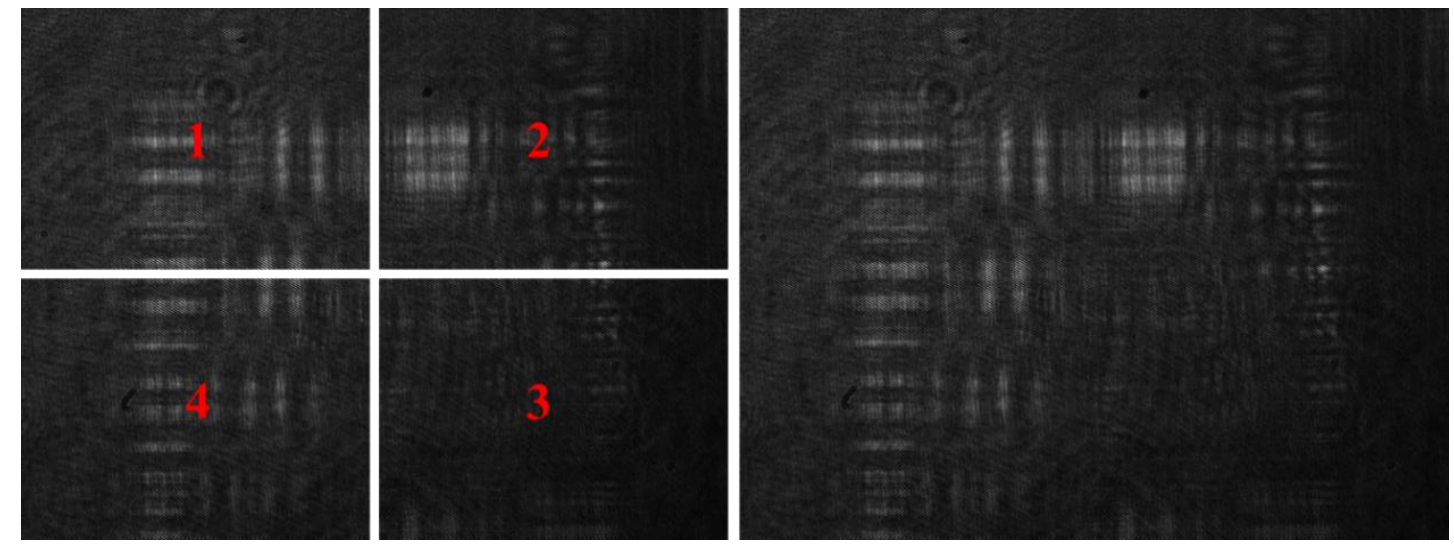

Figure 5.1 Left is segmented sub digital holograms, and right is the synthetic one. 
Numerical reconstruction is applied for all holograms in Fig. 5.1. The reconstructed phases and intensities are shown in Figs. 5.2 and 5.3. From comparison, it can be seen that the synthetic phase and intensity are more resolvable in detail. This also agrees with theoretical analysis.
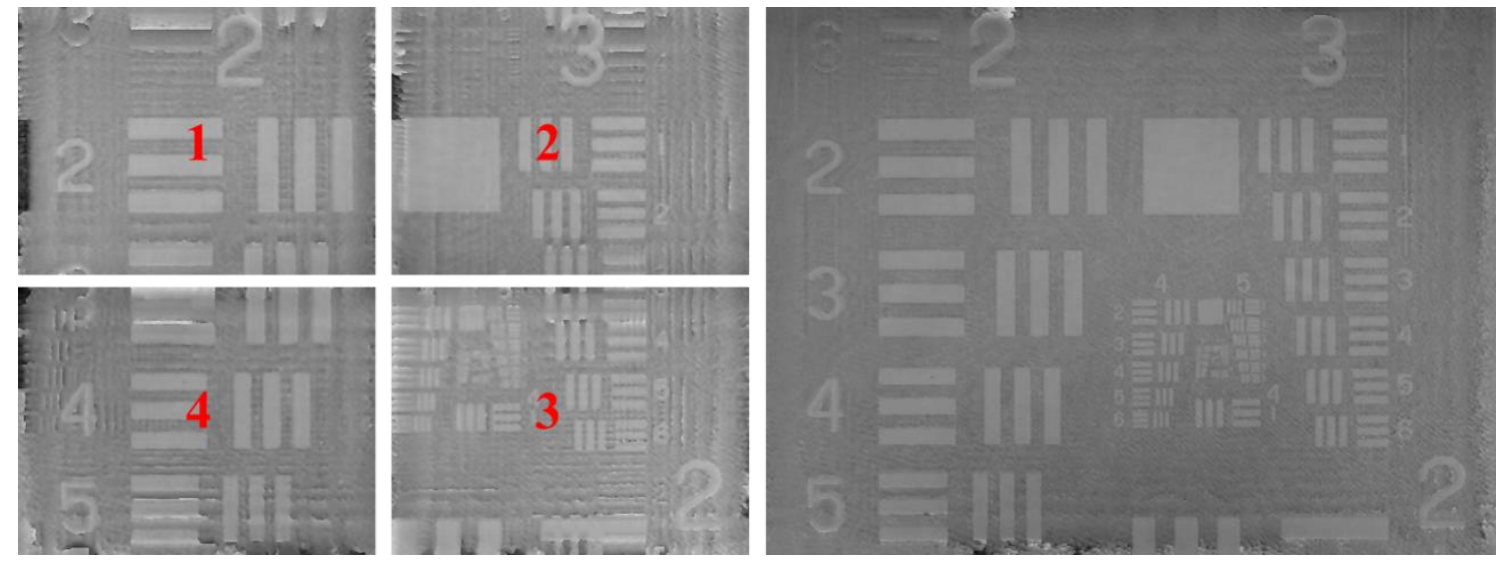

Figure 5.2 Left is reconstructed sub phases, and right is the synthetic one.
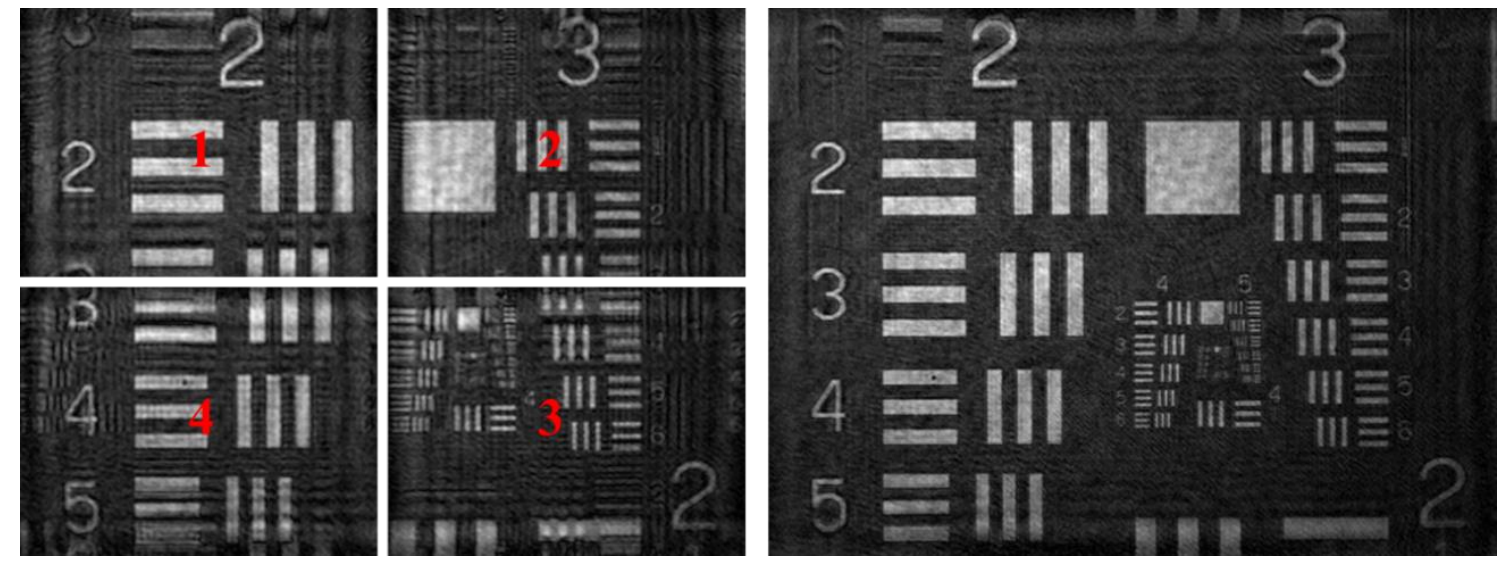

Figure 5.3 Left is the reconstructed sub intensities, and right is the synthetic one.

\subsection{Introduction of stitching algorithms}

The stitched phase is the final desired result in synthetic aperture digital holography.

There are three mainly ways in current technologies to get the phase: one way is to stitch 
the reconstructed phases into one directly; another way is to stitch the digital holograms first, and then reconstruct it to get the phase; the last way is to do the FFT for the digital holograms, and then combines the first order spectrum to reconstruct the phase. Each method has its advantages and disadvantages. The first way is most directly. However, the reconstructed phases are affected by many unpredictable factors, such as phase unwrapping, featureless, spectrum shift, and so on. These may lead to the phases difficult to stitch. Moreover, each phase needs reconstruction separately, the process is timeconsuming. The second way is the fastest one because it only needs once numerical reconstruction. The problem for this way is that it depends on high precision motorized stages to provide accurate composition size. The last way also needs once numerical reconstruction. But this way has the lowest efficiency because digital holograms stitching has a big challenge. Digital hologram consists of many tiny fringes and these fringes are affected by the time shift, air fluctuation, light source stability, and rand noise. The fringes distribution are not robust enough for digital hologram stitching. In this research, we investigate different stitching methods to compare their performances.

There are many researchers paying efforts in synthetic aperture digital holography. In 2001, a synthetic aperture setup with on-axis digital heterodyne holography was reported by Moonseok Kim etc. [125]. Heterodyne is introduced to make the phase difference more stable. The method used in this paper actually enhances the resolution of the digital hologram by multiplexing sub-holograms from different angles. In 2002, off-axis digital holography with a synthetic aperture was reported by Jürgen H. Massig [126]. The synthetic aperture is achieved by moving $\mathrm{CCD}$ at 9 different positions. Later, a short range synthetic aperture off-axis digital holography was introduced by Renaud Binet etc. 
[127]. The principle synthetic aperture is similar to the previous one. The CCD size is $128 \times 128$ pixel which is small compared to current CCD size. In 2007, a synthetic aperture on-line digital holography was presented and applied in testing of sphacelaria alga [128]. Another paper with scanning holographic microscopy mainly discussed the theoretical work in exceeding Rayleigh limit [129]. In 2008, an angular multiplexing digital holography was reported by Caojin Yun etc. [130]. In this paper, three objective beams are employed from different angles. By recording in one shot by $\mathrm{CCD}$, numerical reconstruction with 3 times enhanced the resolution of the intensity distribution. Jianglei Di etc. proposed a synthetic aperture digital holography by scanning with linear CCD camera [131]. This linear CCD camera scanning technique provides us two merits: one is low cost; another one is no stitching process, which means the computation will be simple. However, due to the linear CCD camera size limitation, a normal FOV requires a long time scanning which is only one shot work for common CCD camera. In 2009, a diffraction grating between the object and CCD camera was introduced to enlarge the NA [132]. Numerical correcting the joint misplacement of the sub-holograms in synthetic aperture digital holography was discussed in [133]. A long working distance synthetic aperture Fresnel off-axis digital holography was proposed in [134]. In 2010, application in deformation measurement with synthetic aperture digital holography was reported [135]. A more complicated synthetic aperture digital holograph system was proposed in [136]. In 2011, a spiral trajectory focused spot on back focal plane of condenser lens was introduced to realize multiple angular synthetic apertures [137]. A theoretical work to show resolution improvement by the synthetic aperture in digital Fresnel holography was finished by Yan Hao [138]. A two-step cross-correlation registration method was 
introduced to stitch holograms [139]. Phase correction using sharpness metrics was utilized to achieve diffraction-limited resolution.

\subsection{Principle of stitching algorithm based on first order spectrum}

\subsubsection{Scanning route}

The digital holographic system is often an off-axis interferometric configuration as shown in Fig. 5.4. At image plane, the image size is surely larger than the size of CCD sensor. This, of course, will result in that the image record is only part of the sample. However, the full field of view of the sample is desired. Thus, scanning is required in this situation. In addition, the scanning is finished at the same imaging distance $d$.

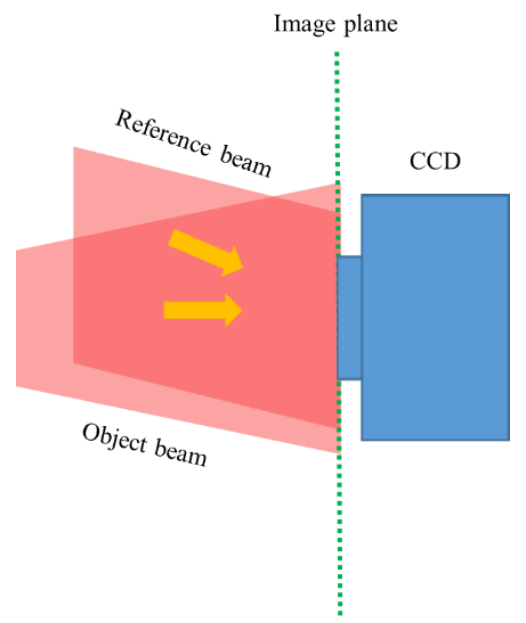

Figure 5.4 Off-axis configuration for imaging of digital holographic system.

A zigzag is used in this research in scanning. Assume the image size is $L x \times L y$, the scanning route goes along the dash blue line as shown in Fig. 5.5. It can be seen that the scanning steps in $x$ or $y$ direction is predefined in scanning. 


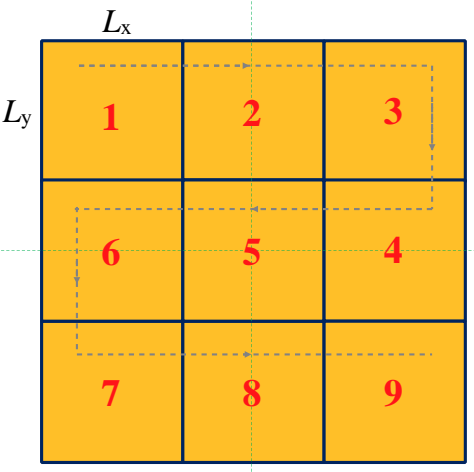

Figure 5.5 Zigzag scanning route.

\subsubsection{Fourier transform and first order spectrum filtering}

Once the digital hologram is captured in each scanning step, FFT is performed

$$
\left.\Im\left\{f_{j}(x, y)\right\}=\Im E_{R}^{*} I_{j}\right\}\left[f_{x}, f_{y}\right]
$$

where $j$ is the index of the image in scanning. $I_{j}$ is the $j^{\text {th }}$ digital hologram.

When the FFT is finished, the first order spectrum is filtered and re-centered in the composition matrix.

\subsubsection{Registration}

The compositing surface size is determined by two factors: scanning steps and step length. The step length is often selected based on the image size. Consider the image degradation in Fourier transform, overlapping in neighbouring images is necessary. Here, we apply $20 \%$ image size in overlapping area to suppress the degradation. The scanning steps actually have no limitation except for the ranges of the motorized stages. Yet we need to consider 
that the actual synthetic image cannot be too large so that the computer is not able to reconstruct it due to memory resource limitation.

$$
\begin{aligned}
\mathfrak{T}\{f(x, y)\} & =\mathfrak{J}\left\{f_{1}\left(x-x_{1}, y-y_{1}\right)\right\}+\mathfrak{J}\left\{f_{2}\left(x-x_{2}, y-y_{2}\right)\right\}+\cdots \mathfrak{I}\left\{f_{n}\left(x-x_{n}, y-y_{n}\right)\right\} \\
& =\sum_{j=1}^{n} \mathfrak{I}\left\{f_{j}\left(x-x_{j}, y-y_{j}\right)\right\}
\end{aligned}
$$

where the $f(x, y)$ is the synthetic optical field of the object. $\left(x_{j}, y_{j}\right)$ is the coordinate that the first order spectrum center locates at the compositing surface.

\subsubsection{Numerical reconstruction}

The optical field of the object can be retrieved by playing inverse FFT on the complex in Eq. 5.3

$$
E_{o}(x, y, d)=\mathfrak{I}^{-}\left\{\mathfrak{I}\{f(x, y)\}\left[f_{x}, f_{y}\right] \exp \left[i \frac{2 \pi}{\lambda} d \sqrt{1-r^{2}}\right] \operatorname{circ}(r)\right\}[x, y]
$$

The phase of the optical field can be obtained

$$
\varphi(x, y)=\arctan \frac{\operatorname{Im}\left[E_{o}(x, y, d)\right]}{\operatorname{Re}\left[E_{o}(x, y, d)\right]}
$$

The intensity distribution gives

$$
I(x, y)=\left|E_{o}(x, y, d)\right|
$$

Now the phase and the intensity distribution of the optical field are obtained. One should note that the phase is wrapped. Phase unwrapping is required to remove the discontinuities. 


\subsection{Experiment and results}

Figure 5.6 shows the schematic of a Mach-Zehnder interferometer for the experimental measurement of an aspheric lens. A collimated laser beam is split by BS1 into two beams. One beam is reflected by the Mirror 1 (M1) and is then normally incident on the aspheric lens surface under test. Since the beam passing through the aspheric lens diverges very fast, a triplet is introduced to reshape the beam which acts as the object beam. The other beam serves as the reference beam and interferes with the object beam at BS2 with a small angular offset between them. The small angular offset is often manually adjusted to ensure the first order spectrum can be separated from the zero order spectrum in frequency domain. At last, the inference pattern is captured by CCD camera.

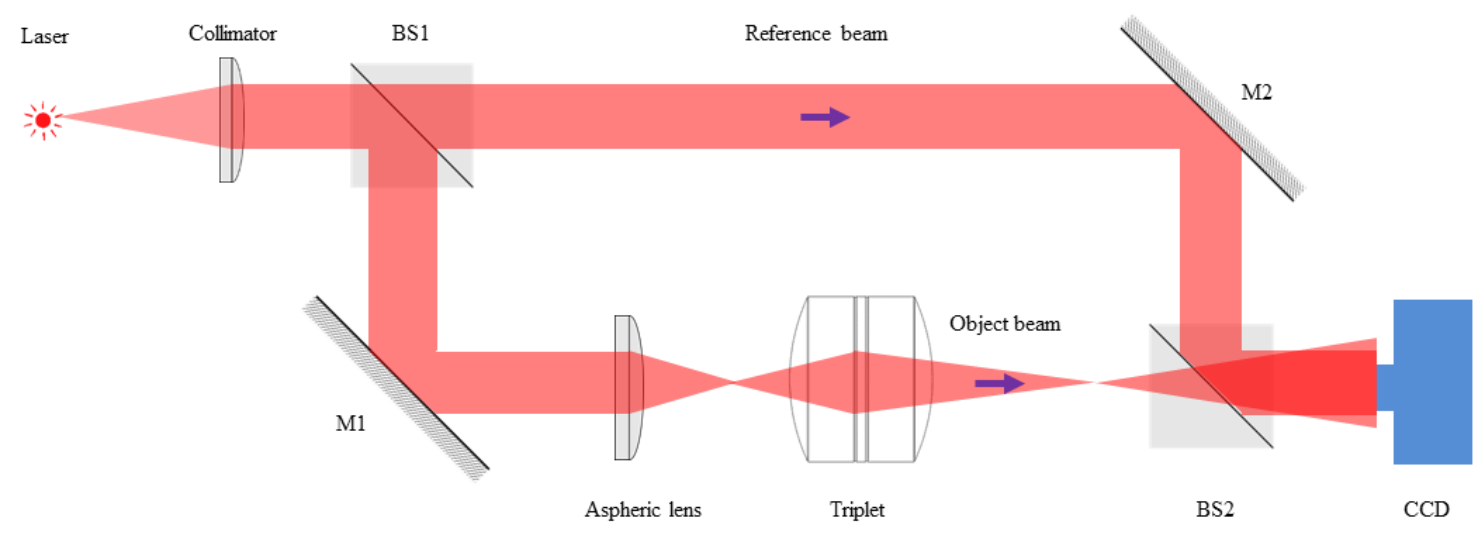

Figure 5.6 Schematic of experimental configuration.

In the experiment, a $660 \mathrm{~nm}$ solid laser was used as light source. The sample under testing is a precision aspheric lens (KPA16) from Newport, with $25.00 \mathrm{~mm}$ diameter and 37.50 $\mathrm{mm}$ focal length. The triplet is mounted Hastings achromatic triplet from Thorlabs, and its model is TRH254-040-A-ML. An 8-bit Imaging Source USB monochromatic CCD camera was used to record the digital hologram. Its resolution is $1280 \times 960$, along with pixel size $4.65 \mathrm{um} \times 4.65 \mathrm{um}$. The distance between the aspheric lens and the triplet is $65 \mathrm{~mm}$, and that of triplet to CCD camera is $270 \mathrm{~mm}$. Two Picomotor actuators with 30 
nm positioning resolution from New Port are used to set up $x-y$ stages for motorizing fine-positioning in scanning. The experimental setup is shown in Fig. 5.7.

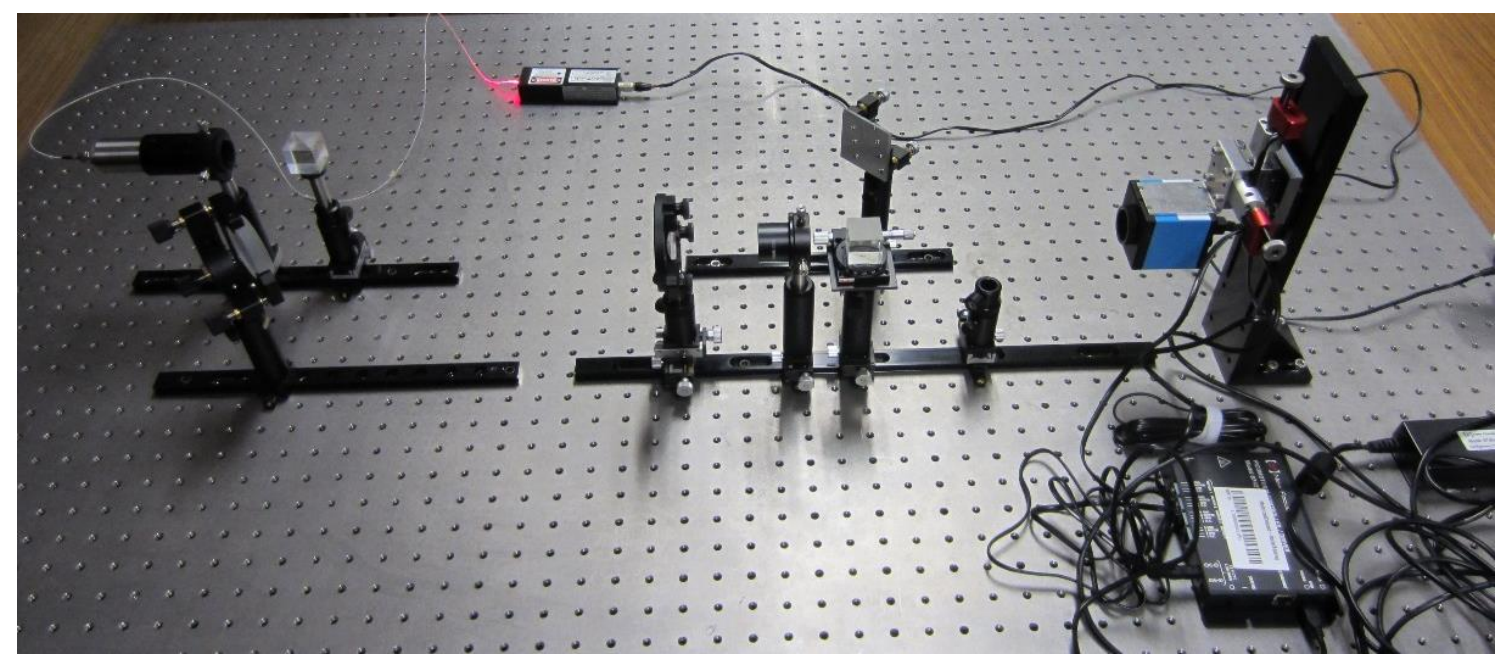

Figure 5.7 Experimental setup of synthetic aperture digital holography.

The scanning route is as described in Fig. 5.5. The steps in $x$ and $y$ directions are selected with 155000 and 139500, respectively. Because each step resolution is $30 \mathrm{~nm}$, the step lengths in scanning are $4.65 \mathrm{~mm}$ and $4.185 \mathrm{~mm}$ for $x$ and $y$ directions, respectively. Each direction has 3 scanning steps which correspond to a $3 \times 3$ scanning grid. Digital holograms were captured and recorded sequentially in Fig. 5.8. The digital holograms are named by scanning sequence with $I_{1}, I_{2}, I_{3}, I_{4}, I_{5}, I_{6}, I_{7}, I_{8}$, and $I_{9}$. 


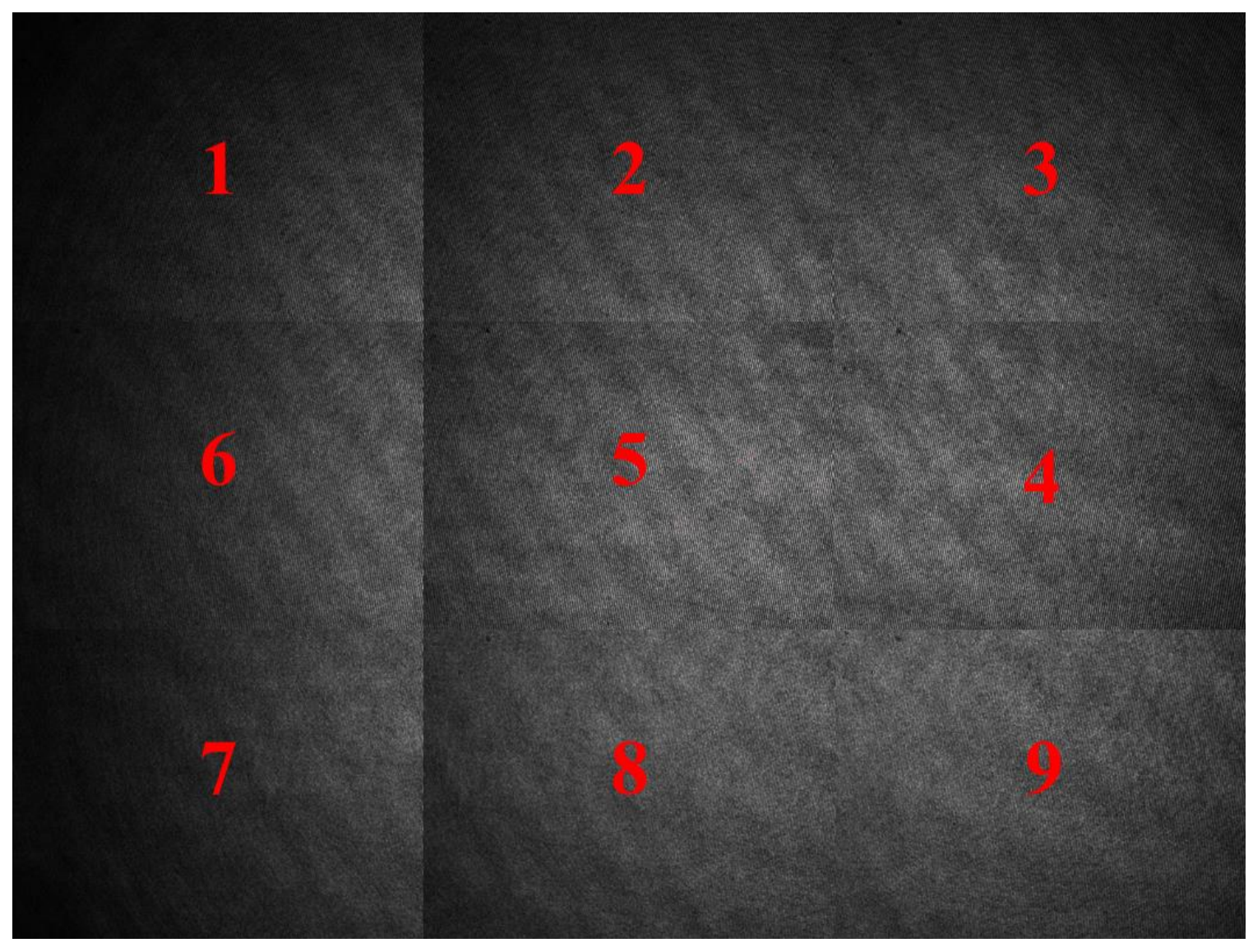

Figure 5.8 9 digital holograms recorded by scanning.

The final image size is $2760 \times 3280$ pixels. The digital holograms are then apodized to recompose on the final image. The first to ninth digital hologram is resize with $I_{1}(1: 930,1: 1140), \quad I_{2}(1: 930,141: 1140), \quad I_{3}(1: 930,141: 1280), \quad I_{4}(31: 930,1: 1140)$, $I_{5}(31: 930,141: 1140), \quad I_{6}(31: 930,141: 1280), \quad I_{7}(31: 960,1: 1140), \quad I_{8}(31: 960,141: 1140)$, $I_{9}(31: 960,141: 1280)$. The apodized digital holograms are sequentially placed on the final image which is padded with zero in the left area. Figure 5.9 shows the registration of the first digital hologram $I_{1}$. The image after registration is named $I_{1}^{\prime}$. The reason why we register the digital hologram one by one is that the following FFT can be processed simultaneously with the scanning process. Of course, one also can prefer to register one 
time for all digital holograms after the scanning is finished. However, this process will cost more time.

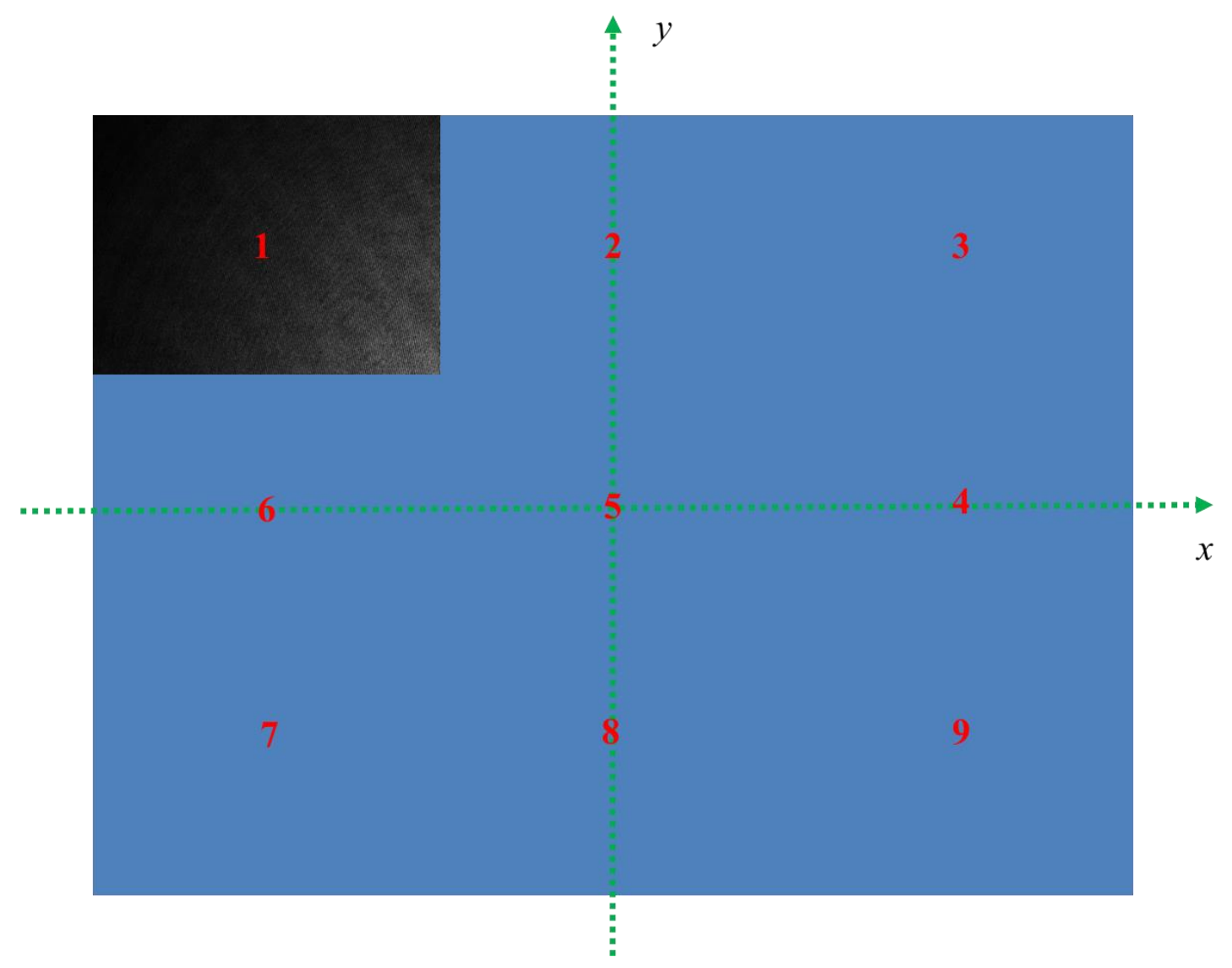

Figure 5.9 The first digital hologram registration

The following process is to do FFT for the image $I_{1}^{\prime}$ in Fig. 5.9. The frequency domain image is shown in Fig. 5.10. As the dash point ellipse circled, the positive first order spectrum is filtered. 


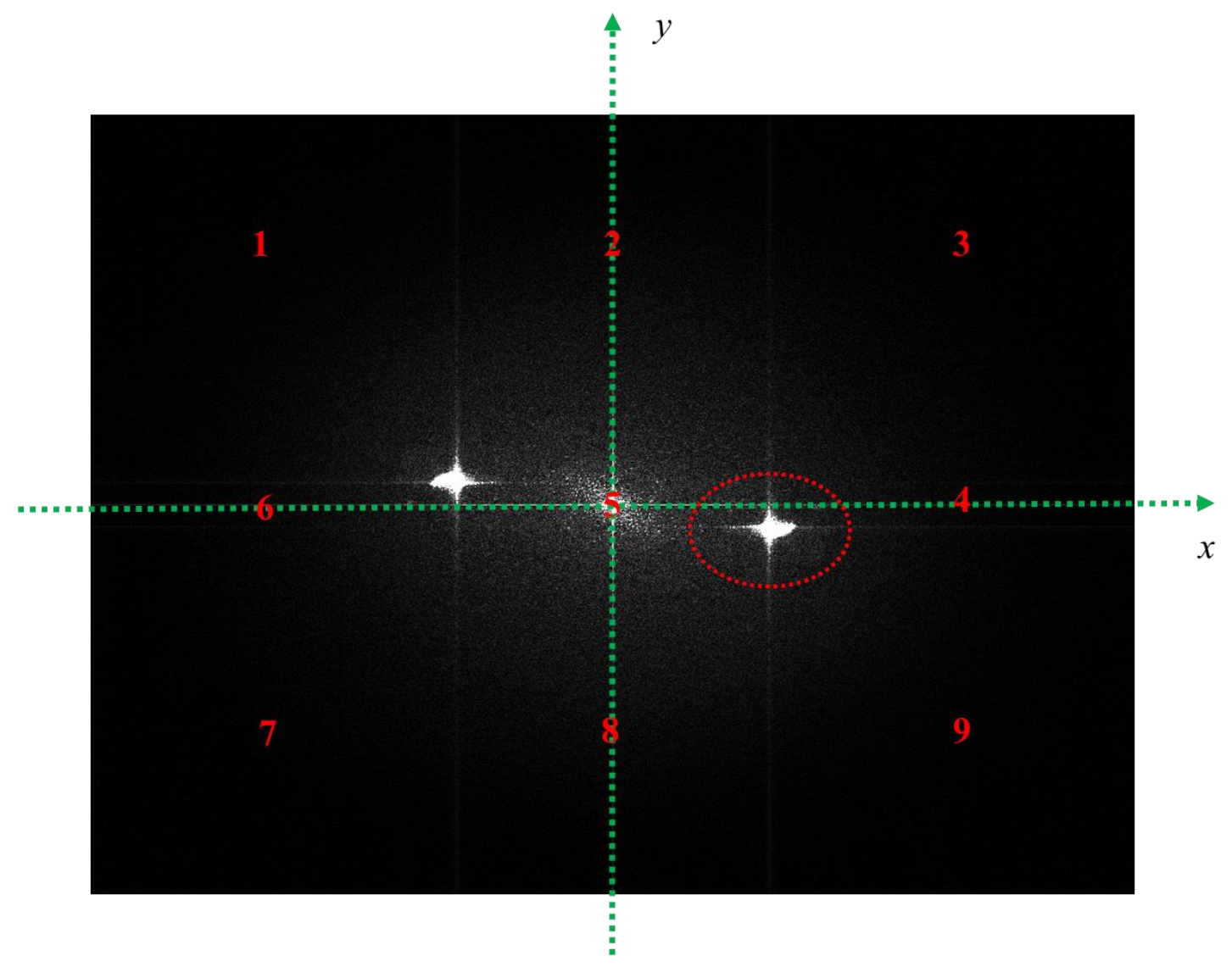

Figure 5.10 FFT of the first digital hologram.

One the first order spectrum is selected, one needs to move the spectrum to the center of the image as shown in Fig. 5.11. As the spectrum is complex, one need to move both real and image parts in operation. Here, only the modulus of the complex is displayed, one must pay attention to this step. 


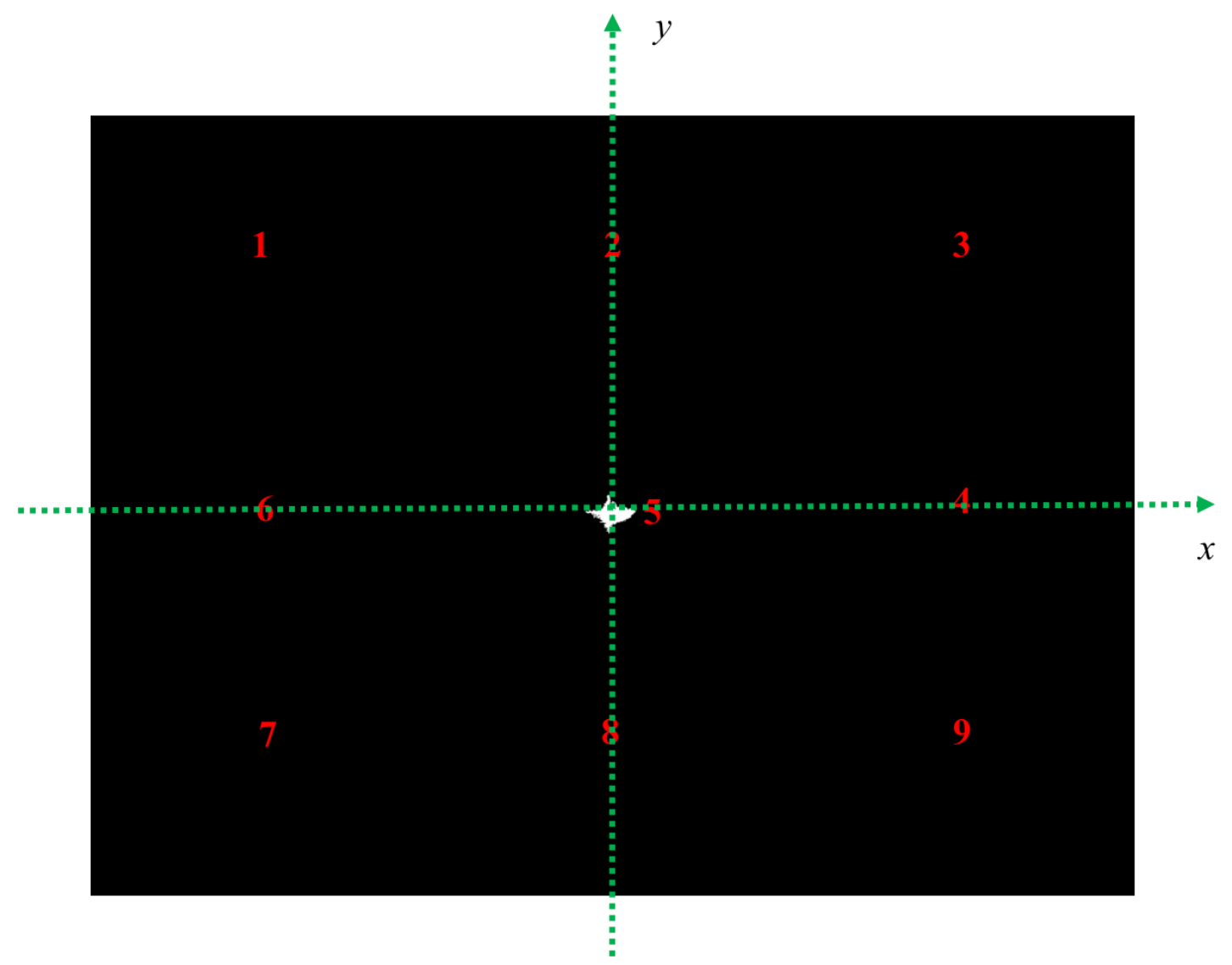

Figure 5.11 Centering of the filtered first order spectrum.

With scanning going on, the first order spectrum from different holograms is obtained and added together. Once the scanning is finished, the phase is computed and shown in Fig. 5.12. It can be seen that the wrapped phases are modulated with different contrasts at the neighboring edges of any two sub images. However, these discontinuities can be removed by phase unwrapping. 


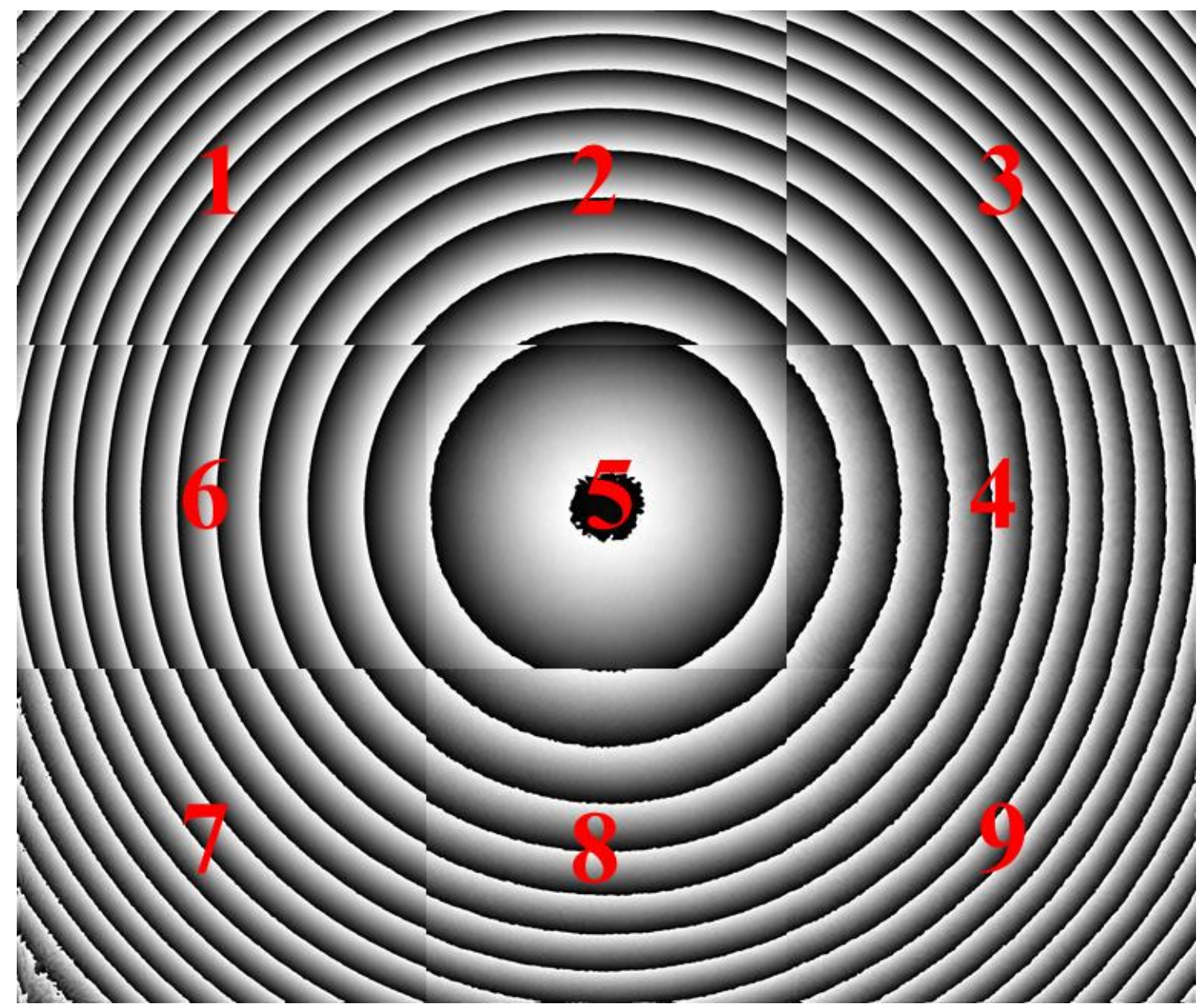

Figure 5.12 Wrapped phase of the complex.

Next, the phase unwrapping is performed on the wrapped phase in Fig. 5.12. The unwrapped phase is shown in Fig. 5.13, and its 3D shape is shown in Fig 5.14. 


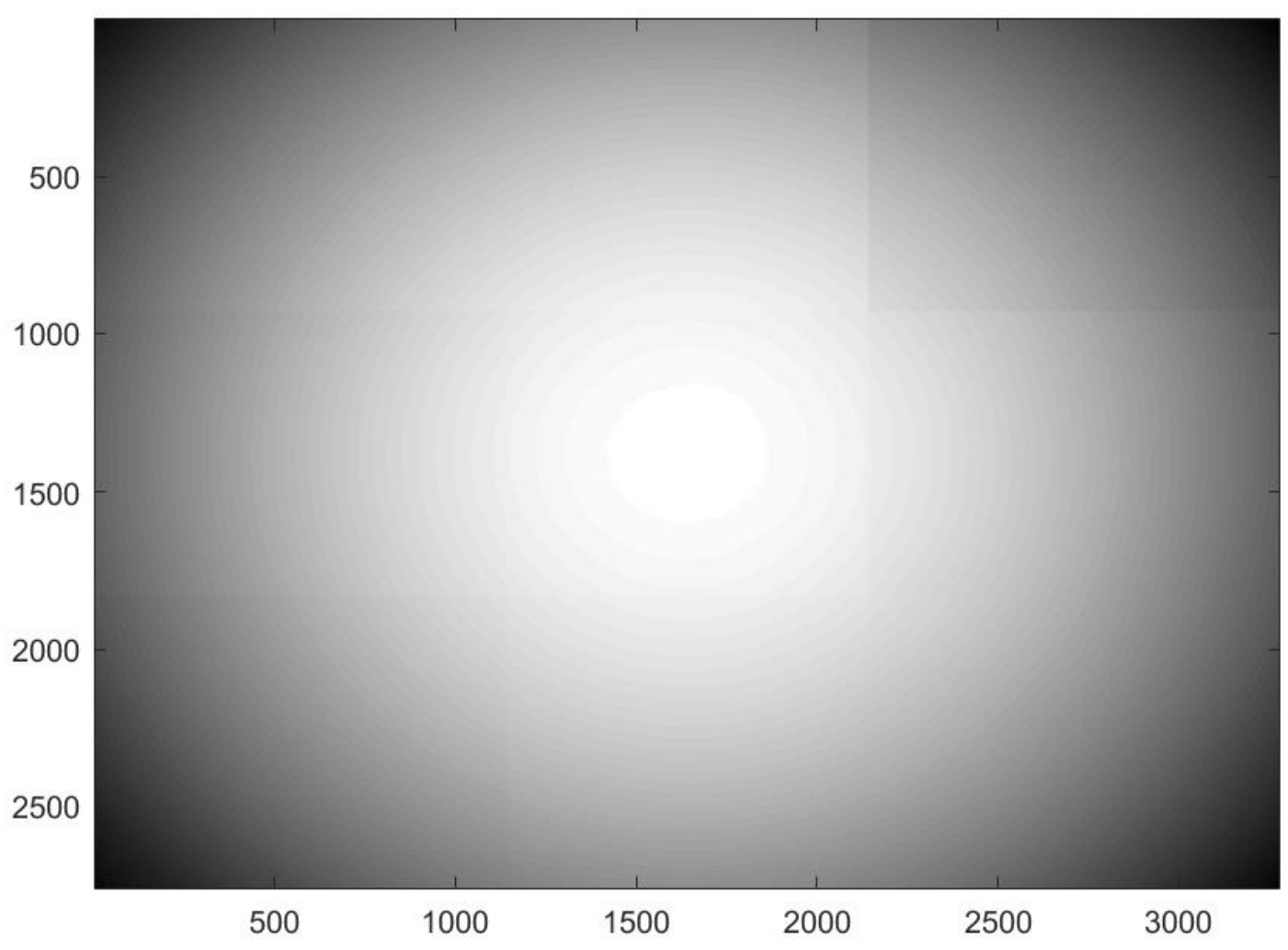

Figure 5.13 Unwrapped phase of the complex.

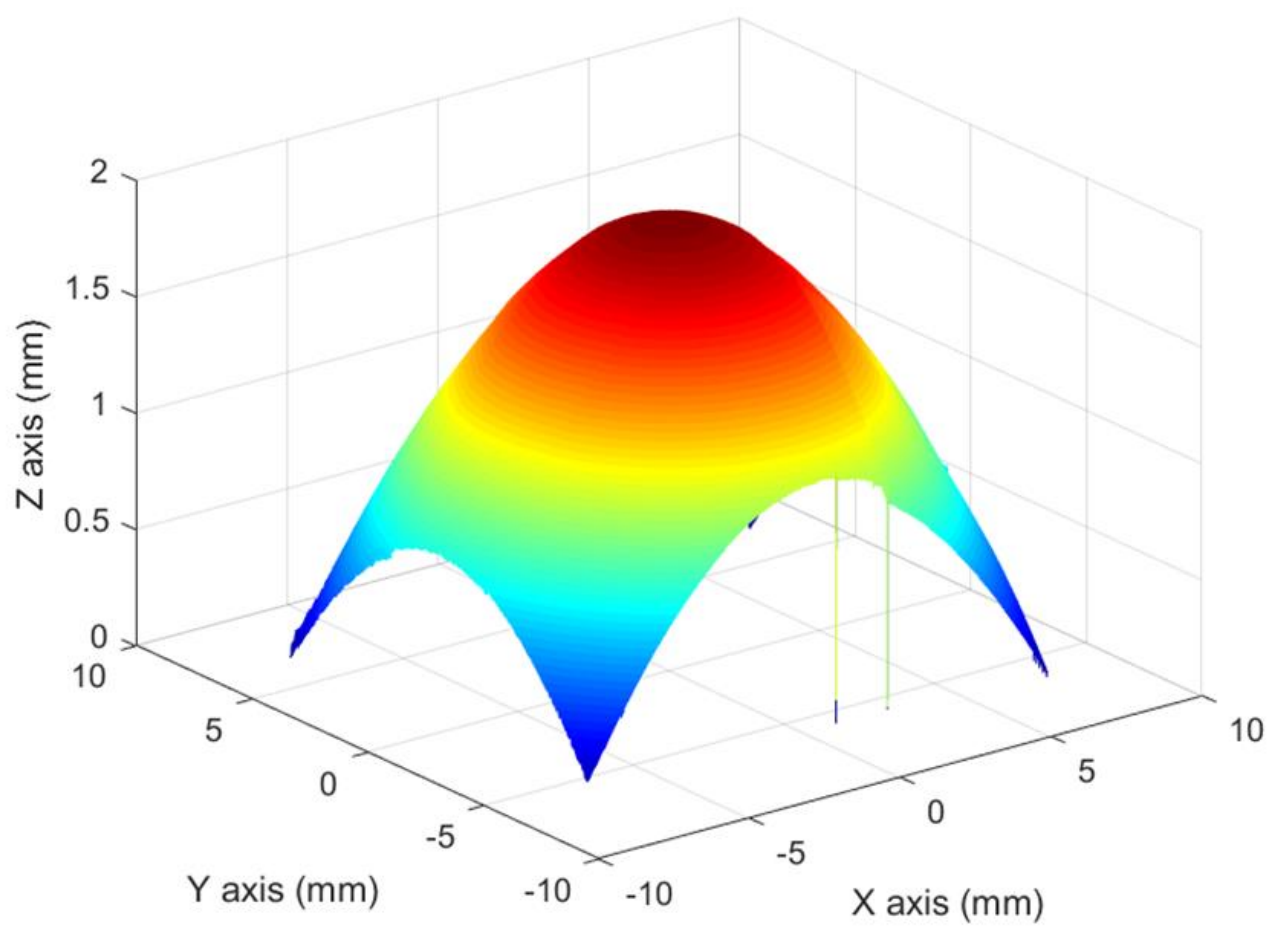

Figure 5.14 3D rendering of the recovered surface of the aspheric lens. 
To compare the reconstruction result with mature stitching method, numerical reconstruction is implemented on digital holograms in Fig. 5.8. The reconstructed phases without apodization are aligned and shown in Fig. 5.15. In these phases, it can be seen that there are discontinuities on their edges in particular.

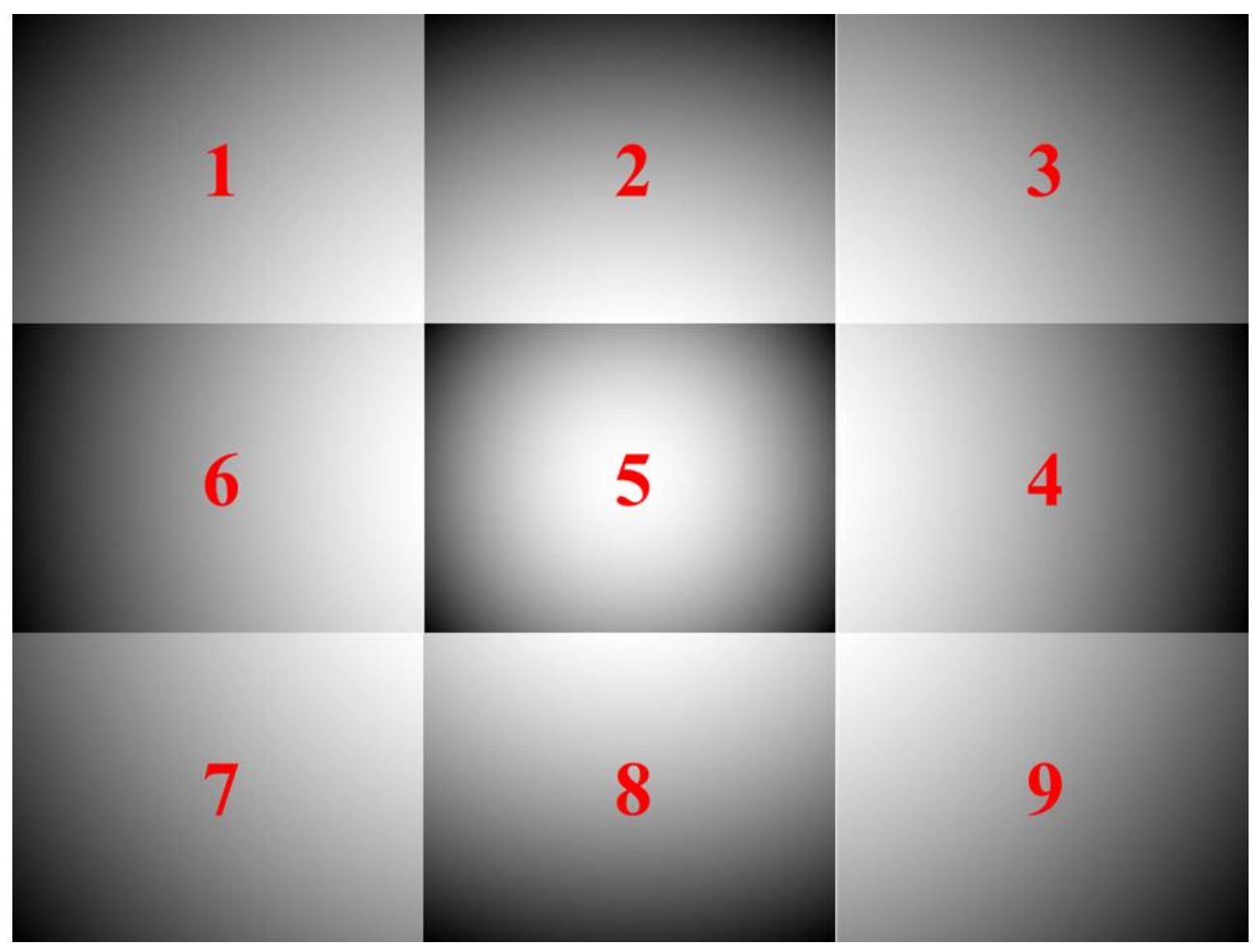

Figure 5.15 Unwrapped phases of 9 digital holograms.

Stitching based on Microsoft software - Image Composite Editor (ICE) [140] - is performed on above phases. $22 \%$ and $7 \%$ overlaps in horizontal and vertical directions are used in alignment, and the stitched phase is shown in Fig. 5.16. The stitched phase is incorrect, and has totally lost its meaning to the aspheric lens. Although this mature method is simple and straightforward, it's ineffective in this kind of phase stitching. 
Moreover, it is a post-processing software, and not suitable for in-line inspection. While the proposed method can do reconstruction as soon as the scanning is finished, which is more attractive in measurement and fabrication.

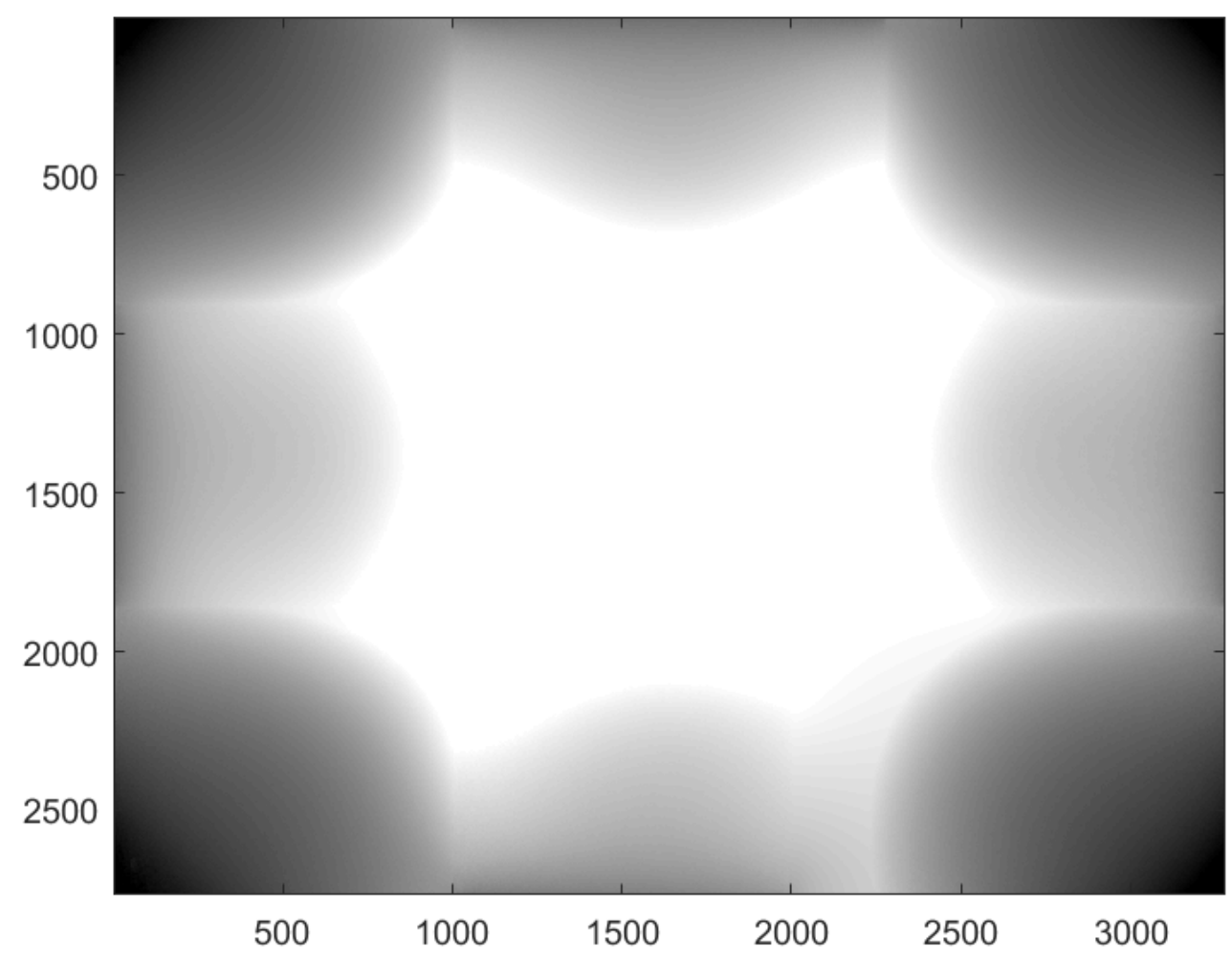

Figure 5.16 Stitched phase.

Another aspect to mention is that the nowadays large-sensor camera (most of them are CMOS camera) is becoming more and more common, and some sensors are larger than 25 mega pixels. This kind of camera can capture several times larger image than the 1 mega pixels CCD camera does. This raises a question: do we still need synthetic aperture technique? The answer is yes. Firstly, consider the building process, the dark noise CMOS is higher than in CCD camera. In precision measurement, CCD camera is still of the first choice owing to its low noise characteristic. Secondly, the proposed method can be applied to large-sensor camera. As a general method, it has no limit to camera size and 
type. Last but not least, there are two reasons to select CCD camera in this research: the dark noise is lower; the price is much lower than large-sensor CMOS camera.

\subsection{Chapter summary}

In this chapter, the resolution is analyzed to be improvable by synthetic aperture digital holography. The digital hologram of synthetic aperture is theoretically analyzed to have more information than sub ones. A USAF target example is used to explain the technique in resolving more detail information.

Next, the first order spectrum based registration is proposed. The registration depends on the high precision motorized stages to compute the size of the composite surface. The method substantially utilizes the numerical reconstruction process and does not need full numerical reconstruction of sub digital holograms which greatly improves running efficiency.

The experiment is done to verify the proposed method. The aspheric lens is tested in Mach-Zehnder interferometer and then scanned by $3 \times 3$ matrices to achieve synthetic aperture digital holography. The numerical reconstruction is performed once after all the first order spectrums are summed. Phase unwrapping is used to remove the discontinuities in the wrapped phase. By comparison with the stitched result by ICE, it shows the proposed method is simple and efficient. 


\section{ASPHERIC LENS CHARATERIZATION}

In this chapter, aspheric lens characterization is discussed. In factory, once the measurement is finished, the data related to its tomography is also available. As the aspheric surface expression is nonlinear, the design parameters are difficult to access to. Some researchers turn their focuses onto tolerancing, while some proposed different polynomials instead of standard one. Since the design parameters are so important for designers, in particular, they depend on them to improve the aspheric lens performance. Tolerancing can only justify whether the fabrication result falls in wanted range, but cannot provide any usefully information for designers. Therefore, the parameters are desired. We propose a simplified expression for computing the parameters.

On other hand, aberration in form of wavefront is available in some metrologies. Zernike polynomials can represent aberrations with significant meaning. Moreover, they are orthogonal to each other which is very important in fitting. A modified Zernike polynomials fitting method is proposed and applied in wavefront evaluation.

\subsection{Aspheric surface fitting}

The performance of high precision optical system using spherical optics is inevitably limited by optical aberrations $[141,142]$. To correct these aberrations, lens combinations are often used. As a result, the cost, space, and weight of the system will increase simultaneously. By using aspheric optical lens, optical aberrations can be reduced or even eliminated with fewer elements resulting in savings in cost, space and weight [1]. Once the measurement data of an aspheric surface is obtain, due to the nonlinearity in its 
mathematical expression, accurate and rapid characterization of the data is still challenging.

The aim of aspheric surface fitting is to determine the parameters of a measured aspheric surface. Research regarding the surface fitting method has been broadly investigated. Most of the common algorithms for conic fitting were studied by Zhang [143]. A general aspheric surface includes not only conic term but also the even higher order terms. In addition, the conic fitting itself is not compatible for aspheric surface fitting. The best-fit sphere algorithm was used to extract the spherical diameter in some simple situations [144, 145]. Simplified expressions or alternatives have been proposed to fit an aspheric surface, however, accuracy is the main problem here [146-149]. A projection from polynomial equations to vector space was used to avoid the ill-condition in the problem [150]. The Levenberg-Marquartdt (LM) algorithm was explored to fit aspheric surface [17] although the accuracy of the algorithm is far from satisfactory. The Limited memory-Broyden-Fletcher-Goldfarb-Shannon (L-BFGS), LM and a variant of the Iterative Closest Point (ICP) have also been proposed [151]. A linear expression was first deduced to obtain initial values for subsequent nonlinear fitting [152]. As the method depends on initial values, when the even order terms go up, the complexity of linear expression will increase greatly. Although it can start with arbitrary values, convergence problem will rise accordingly. Rearrange Zernike polynomials are proposed to fit to make use of their orthogonality. A new orthogonal expression is proposed to replace the standard expression [146, 153-157]. These two method have advantages in fitting because the polynomials they employ are orthogonal. While the disadvantage is their 
parameters lose original meanings. Unless the standard is changed, their parameters have little help to designers.

Here, we propose a new aspheric surface fitting method with discrete orthogonal polynomials. The proposed method expands the quadratic part of an aspheric surface into a series of even order terms by binomial expansion. The number of these terms is purposely chosen at least two more than that in design. After combining with designed even order terms, a new aspheric surface expression which only contains even order terms is thus obtained. As these terms are not orthogonal to each other, Gram-Schmitt orthogonalization is applied on them to generate a new set of discrete orthogonal polynomials. Least squares method is used in fitting then. Through simulation and experimental testing, the fitting results are in good agreement with what were designed. Owing to the orthogonality and least square method, the proposed method also shows good convergence speed.

The basic idea for aspheric surface fitting is first to expend the aspheric surface expression into even order polynomials by binomial expansion, and then to combine with existing terms. Through Gram-Schmitt orthogonalization, the new generated terms are converted to discrete orthogonal polynomials. Least squares method is then used to fit data under estimation. Finally, the aspheric surface parameters are deduced.

\subsubsection{Conversion of aspheric surface expression}

The standard ISO 10110-Part 12 describes mathematical representation of aspheric surface as 


$$
z=f(r)=\frac{\frac{r^{2}}{R}}{1+\sqrt{1-(1+\kappa)\left(\frac{r}{R}\right)^{2}}}+\sum_{i=2}^{n} A_{2 i} r^{2 i}
$$

Where $r$ is the lateral coordinate, $z$ is the sag, and $R$ the paraxial surface radius. The conic constant $\kappa$ is 0 for spheres, -1 for parabolas, $<-1$ for hyperbolas, between -1 and 0 for oblate and $>0$ for prolate ellipses.

Substituting $c=1 / R$ and $m=1+\kappa$ into Eq. (6.1) gives the sag as:

$$
z=\frac{c r^{2}}{1+\sqrt{1-m c^{2} r^{2}}}+\sum_{i=2}^{n} A_{2 i} r^{2 i}
$$

Next rationalizing the denominator gives

$$
\begin{aligned}
z & =\frac{c r^{2}}{1+\sqrt{1-m c^{2} r^{2}}}+\sum_{i=2}^{n} A_{2 i} r^{2 i} \\
& =\frac{c r^{2}\left(1-\sqrt{1-m c^{2} r^{2}}\right)}{\left(1+\sqrt{1-m c^{2} r^{2}}\right)\left(1-\sqrt{1-m c^{2} r^{2}}\right)}+\sum_{i=2}^{n} A_{2 i} r^{2 i} \\
& =\frac{c r^{2}\left(1-\sqrt{1-m c^{2} r^{2}}\right)}{1-\left(1-m c^{2} r^{2}\right)}+\sum_{i=2}^{n} A_{2 i} r^{2 i} \\
& =\frac{c r^{2}\left(1-\sqrt{1-m c^{2} r^{2}}\right)}{m c^{2} r^{2}}+\sum_{i=2}^{n} A_{2 i} r^{2 i} \\
& =\frac{1}{m c}\left(1-\sqrt{1-m c^{2} r^{2}}\right)+\sum_{i=2}^{n} A_{2 i} r^{2 i}
\end{aligned}
$$

Eq. (6.3) has two parts - the first is the quadratic surface expression, and the second is a combination of even order polynomials. It is difficult to evaluate an aspheric surface due to the first part which makes the regression nonlinear. To use a linear regression to characterize the aspheric surface, a binomial expansion of the square root of $\left(1-m c^{2} r^{2}\right)$ is used, with the number of terms two more than $n$. 


$$
\begin{aligned}
\frac{1}{m c}\left(1-\sqrt{1-m c^{2} r^{2}}\right) & =\frac{1}{m c}\left\{1-\left[1+\frac{1}{2}\left(-m c^{2} r^{2}\right)-\frac{1}{2 \cdot 4}\left(-m c^{2} r^{2}\right)^{2}+\frac{1 \cdot 3}{2 \cdot 4 \cdot 6}\left(-m c^{2} r^{2}\right)^{3}-\cdots\right]\right\} \\
& =\frac{1}{2} c r^{2}+\frac{1}{2 \cdot 4} m c^{3} r^{4}+\frac{1 \cdot 3}{2 \cdot 4 \cdot 6} m^{2} c^{5} r^{6}+\cdots \\
& =\sum_{i=1}^{n+2}\left(\begin{array}{l}
\frac{1}{2} \\
i
\end{array}\right) m^{i-1} c^{2 i-1} r^{2 i}
\end{aligned}
$$

Thus a new combination of even order polynomials is obtained. Substituting Eq. (6.4) into Eq. (6.3), turns the aspheric surface expression into a linear series of even order polynomials:

$$
\begin{aligned}
z & =\sum_{i=1}^{n+2}\left(\begin{array}{c}
\frac{1}{2} \\
i
\end{array}\right) m^{i-1} c^{2 i-1} r^{2 i}+\sum_{i=2}^{n} A_{2 i} r^{2 i} \\
& =\frac{1}{2} c r^{2}+\sum_{i=2}^{n}\left(\left(\begin{array}{c}
\frac{1}{2} \\
i
\end{array}\right) m^{i-1} c^{2 i-1}+A_{2 i}\right) r^{2 i}+\sum_{i=n+1}^{n+2}\left(\begin{array}{c}
\frac{1}{2} \\
i
\end{array}\right) m^{i-1} c^{2 i-1} r^{2 i}
\end{aligned}
$$

Here, for convenience, Eq. (6.5) is rewritten as:

$$
z=\sum_{i=1}^{n+2} a_{i} U_{i}
$$

where $U_{i}=r^{2 i}$, and $a_{i}$ is the coefficient.

\subsubsection{Gram-Schmitt orthogonalization}

Despite reducing the sag to a linear sum of even order polynomials, Eq. (6.6), fitting the surface with these polynomials is not straightforward. These polynomials are not orthogonal to each other, resulting in non-uniqueness of solution as well as convergence 
problem. Thus, the Gram-Schmidt orthogonalization is used to construct a set of polynomials $V_{j}$ which are orthogonal in Cartesian space. For convenience, let $L=n+2$

$$
z=\sum_{j=1}^{L} b_{j} V_{j}
$$

Then, for Gram-Schmidt orthogonalization [158], $V_{j}$ is computed as:

$$
V_{j}=U_{j}+\sum_{i=1}^{j-1} c_{j, i} U_{i}
$$

In Cartesian space, the polynomials $V_{j}$ satisfy the following condition:

$$
\sum V_{j} V_{p}=0
$$

And $c_{j, i}$ can be written as:

$$
c_{j, i}=\sum_{l=1}^{j-1}\left[c_{j-l, i}\left(\sum U_{j} V_{j-l} /\left(\sum V_{j-l}^{2}\right)\right]\right.
$$

Where $i=1,2,3 \ldots j-1$, and $c_{j, j}=1$.

\subsubsection{Least squares method}

Assume the measured aspheric surface data is $W$, then the deviation of fitting can be determined in a least square sense as:

$$
\delta^{2}=\sum\left(W-\sum_{j=1}^{L} b_{j} V_{j}\right)^{2}
$$

To minimize $\delta^{2}$, its differential is set to zero to give:

$$
\frac{\partial \delta^{2}}{\partial b_{j}}=\frac{\partial \sum\left(W-\sum_{j=1}^{L} b_{j} V_{j}\right)^{2}}{\partial b_{j}}=0
$$

The coefficients $b_{j}$ can be obtained by solving above equation. 


$$
b_{j}=\frac{\sum W V_{j}}{\sum V_{j}^{2}}
$$

Where $j=1,2,3 \ldots L$.

Since the coefficients $b_{j}$ and $c_{j i}$ are known, $a_{j}$ can be deduced by substituting Eq. (6.7) and Eq. (6.8) into Eq. (6.6)

$$
W=b_{1} U_{1}+\sum_{j=2}^{L} b_{j}\left(U_{j}+\sum_{i=1}^{j-1} c_{j i} U_{i}\right)
$$

Comparing with Eq. (6.1), the coefficients $a_{j}$ can be obtained as:

$$
a_{j}=b_{j}+\sum_{i=j+1}^{L} b_{i} c_{i j}
$$

where $j=1,2,3 \ldots L-1$ and $a_{L}=b_{L}$.

Once all the parameters of the aspheric surface are determined, the residue of fitting may still be too big. Therefore, iteration is needed. The residue $W^{\prime}$ is updated as:

$$
W^{\prime}=W-\sum_{i=1}^{n} a_{i} U_{i}
$$

And repeating the fitting procedure to get the updated set of coefficients as:

$$
a_{i}=a_{i}+a_{i}^{\prime}
$$

\subsubsection{Aspheric surface parameters}

Since the fitting is implemented in orthogonal space, the fitting will converge after a few iterations. The final fitting result is in the form of coefficients of even order polynomials.

The parameters in Eq. (6.1) are what are really required for characterization. As $a_{1}=\frac{c}{2}$ is known, and the radius $R$ is expressed as

$$
R=\frac{1}{2 a_{1}}
$$


As the designed aspheric surface has limited designed even order terms, in determining $m$, Eq. (6.4) has to contain at least two higher orders than the designed even orders. There are two ways to calculate $m$. The first is to decide whether $n+1$ or $n+2$ is odd, and then to use odd value to calculate $m$ as:

$$
m=\left(\frac{a_{n+1}}{\left(\begin{array}{c}
\frac{1}{2} \\
n+1
\end{array}\right) c^{2 n+1}}\right)^{\frac{1}{n+1}} \text { or } m=\left(\frac{a_{n+2}}{\left(\begin{array}{c}
\frac{1}{2} \\
n+2
\end{array}\right) c^{2 n+3}}\right)^{\frac{1}{n+2}}
$$

Another way to calculate $m$ is to take the ration of the higher order term with the lower order term:

$$
\frac{a_{n+2}}{a_{n+1}}=\frac{\left(\begin{array}{c}
\frac{1}{2} \\
n+2
\end{array}\right) c^{2 n+3} m^{n+2}}{\left(\begin{array}{c}
\frac{1}{2} \\
n+1
\end{array}\right) c^{2 n+1} m^{n+1}}
$$

Simplifying gives

$$
m=\frac{\left(\begin{array}{c}
\frac{1}{2} \\
n+1
\end{array}\right) c^{2} a_{n+2}}{\left(\begin{array}{c}
\frac{1}{2} \\
n+2
\end{array}\right) a_{n+1}}
$$

Once $c$ and $m$ are obtained, the separation of coefficient $A_{2 i}$ in Eq. (6.5) can be readily realized.

$$
A_{2 i}=a_{i}-\left(\begin{array}{c}
\frac{1}{2} \\
i
\end{array}\right) m^{i-1} c^{2 i-1}
$$

Where $i=2 \ldots n$. 


\subsubsection{Algorithm testing}

The algorithm has been applied to an ideal aspheric surface generated by a computer. Random generated parameters were tested for accuracy as well as convergence speed.

The first aspheric surface tested was designed with 3 even order terms as shown in table 6.1. The clear aperture diameter is $4.5 \mathrm{~mm}$. In computation, we used $1000 \times 1000$ sampling grid. The minimum unit size is $4.5 \mu \mathrm{m} \times 4.5 \mu \mathrm{m}$. On 32-bit Windows 7 system with Intel(R) Core ${ }^{\mathrm{TM}}$ i7-3630QM processor, 10 iteration loops were tested. The fitted parameters are listed in table 6.1 for comparison. We can see the fitted parameters are in good agreement with the designed values. To demonstrate more visually, an image of designed aspheric surface is shown Fig. 6.1(a) and rendered in Fig. 6.1(b). The reconstructed aspheric surface based on fitted parameters and the residue of designed and reconstructed surfaces are shown in Fig. 6.1 (c) and (d) respectively. The mean value of residue is $2.603 \mathrm{E}-07 \mathrm{~mm}$, and its standard deviation value is $5.707 \mathrm{E}-07 \mathrm{~mm}$. The line profiles along a selected line as shown in Fig. 6.1(a) and (c) are compared in (e). From the comparison, the proposed method shows good agreement in detail.

Table 6.1 Simulated aspheric surface parameters comparison.

\begin{tabular}{lccclc}
\hline \multicolumn{1}{c}{ Aspheric parameters } & A04 & A06 & A08 & Radius (mm) & Conic Constant \\
\hline Designed & $3.255 \mathrm{E}-3$ & $8.501 \mathrm{E}-5$ & $-2.227 \mathrm{E}-5$ & -2.33 & -0.881 \\
Fitted & $3.243 \mathrm{E}-3$ & $8.475 \mathrm{E}-5$ & $-2.227 \mathrm{E}-5$ & -2.33 & -0.882 \\
\hline
\end{tabular}


(a)

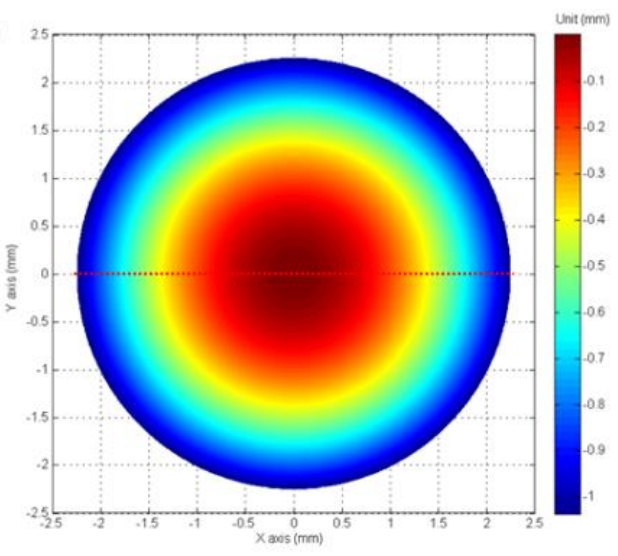

(c)

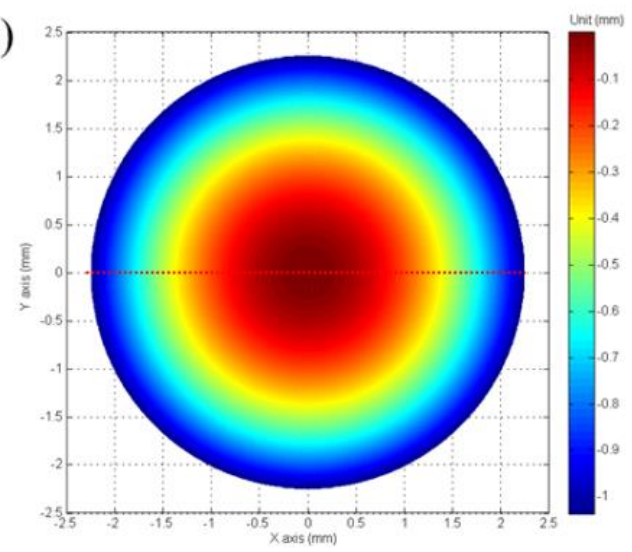

(b)

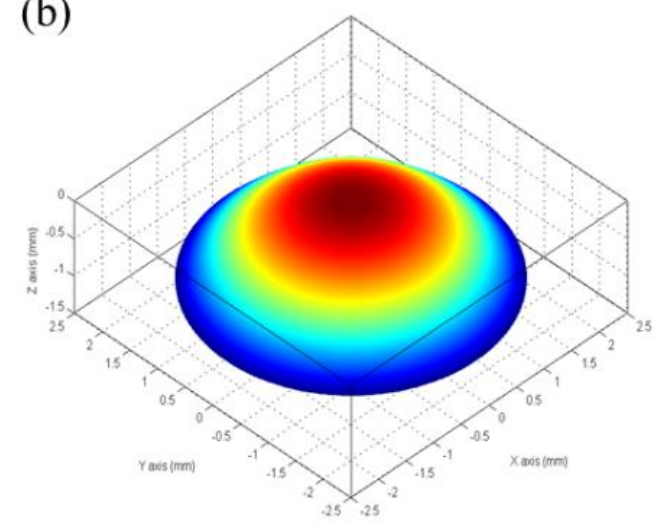

(d)

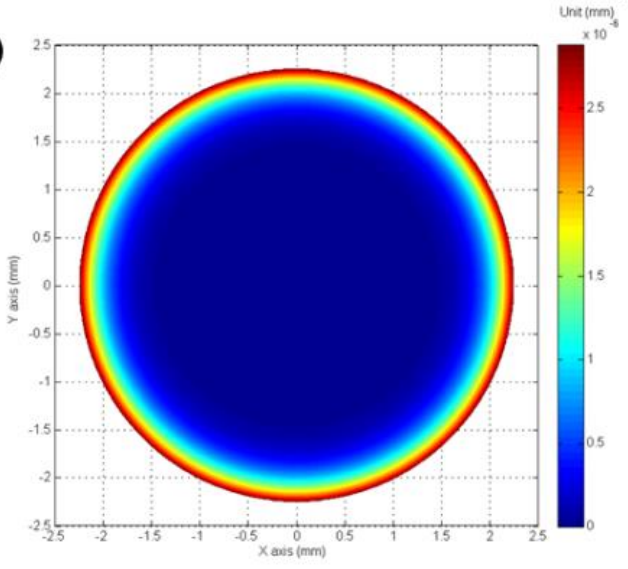

(e)

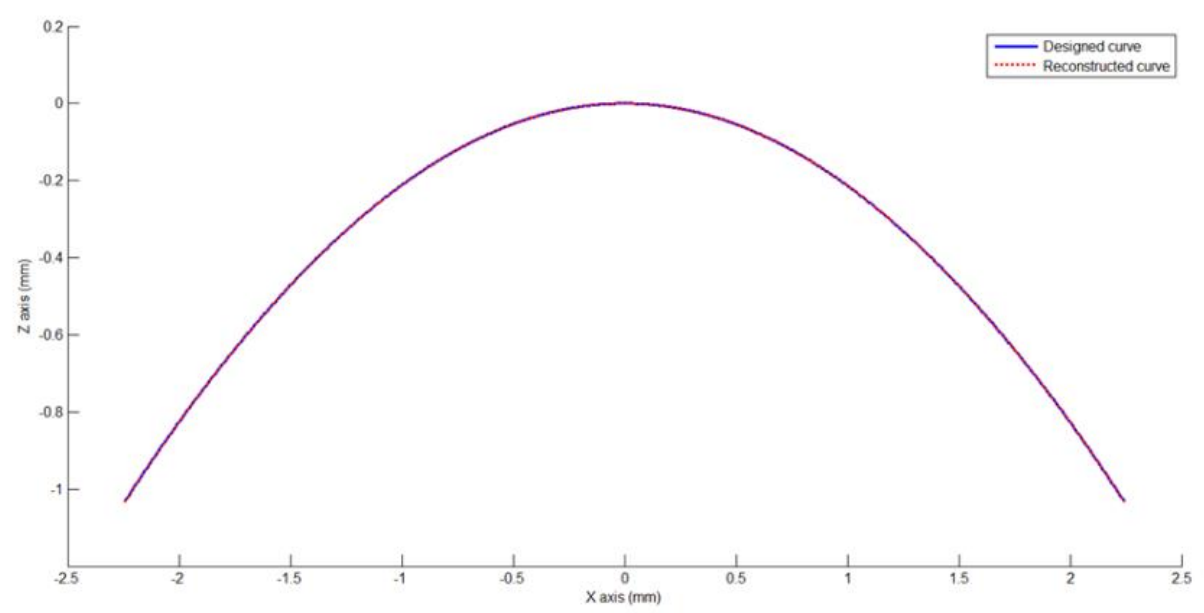

Figure 6.1 (a-b) Designed asphercial surface and its 3D rendering. (c) Reconstrcted asphercial surface. (d) Residue surface. (e) Profile comparison of $500^{\text {th }}$ line betweed designed and reconstructed curves.

The fitting scheme has been shown to provide very close similarity with the designed sag.

Next, the convergence of the fitting is explored. Owing to the orthogonality of the fitting polynomials, not only the uniqueness of fitting result, but also fast convergence is 
ensured. The residue mean and the standard deviation are used to investigate the convergence of the method. Figure 6.2 shows the curves generated from the testing. It is seen that both converge after 4 iteration cycles.

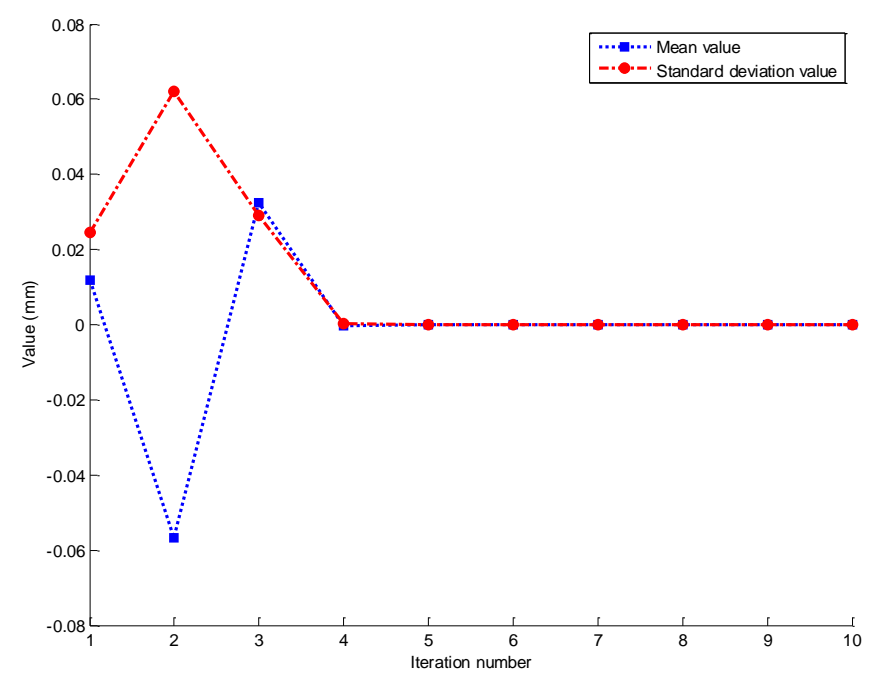

Figure 6.2 Convergence testing.

\subsubsection{Experimental testing}

To further demonstrate the proposed method, experiment by digital holography was carried out [2]. Figure 6.3 shows the experimental arrangement of Mach-Zehnder interferometer[3]. Light from the laser source is spatial filtered and collimated to form a planar beam. The beam is split by a beam splitter (BS1) into two beams: one beam is transmitted through the aspheric lens and imaged by a triplet subsequently, which forms the object beam; the other beam serves as the reference beam. The two beams then meet and interfere at the beam splitter (BS4) with a small angular offset between them. A charge-coupled device (CCD) camera is used to record the interference image-digital hologram. 
In the experiment, a $660 \mathrm{~nm}$ solid laser was used as light source. The sample under testing is a precision aspheric lens (KPA16) from Newport, with $25.00 \mathrm{~mm}$ diameter and 37.50 $\mathrm{mm}$ focal length. The triplet is mounted Hastings achromatic triplet from Thorlabs, and its model is TRH254-040-A-ML. An 8-bit Imaging Source USB monochromatic CCD camera was used to record the digital hologram. Its resolution is $1280 \times 960$, with pixel size $4.65 \mathrm{um} \times 4.65 \mathrm{um}$. The distance between the aspheric lens and the triplet is $65 \mathrm{~mm}$, and that of triplet to CCD camera is $476 \mathrm{~mm}$.

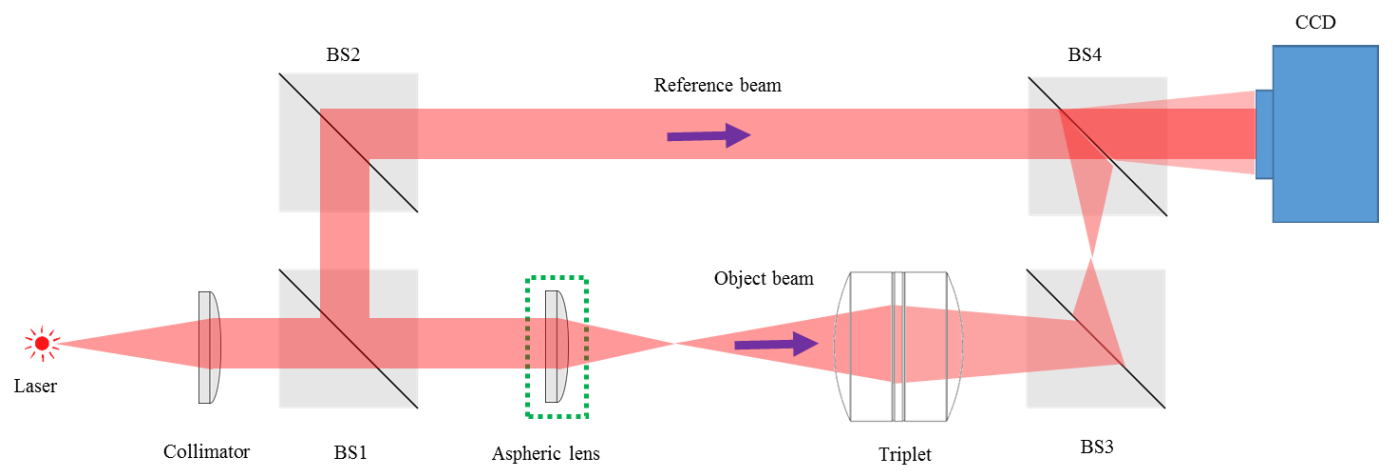

Figure 6.3 Schematic of experimental setup.

In Fig. 6.4, (a) is the digital hologram recorded by the proposed setup, and corresponding numerical reconstruction was implemented by commercial software-Holoscope from d'Optron Pte Ltd. The reconstructed phase was apodized into circle aperture for the following evaluation as shown in (b). Trough calibration, the diameter is $10.875 \mathrm{~mm}$. The circle aperture was normalized into a unit circle for convenience. After 10 iteration loops, the aspheric parameters were obtained and listed in table 6.2 for comparison. Both designed and fitted parameters are in good agreement. As the coefficient goes weaker with the term order increasing, the accuracy of fitting also goes lower. Once very weak coefficient is applied in design, many factors such as fabrication, measurement scheme, system noise, fitting algorithm and computing errors may affect the final characterization 
result. One should pay attention to every step but not only focus on the fitting algorithm. The reconstructed phase by fitted parameters is shown in Fig. 6.4(c). Moreover, the residue of fitting is displayed in Fig. 6.4 (d). The variance in the residue is mainly caused by random noise and irregularity of optical surface in the whole system. To compare the fitting goodness, a plot along the $480^{\text {th }}$ line in Fig. 6.4(b) and (c) are drawn in (e). The plot shows good agreement of fitting result to the experimental data.

Table 6.2 Experimental aspheric surface parameters comparison.

\begin{tabular}{lllllll}
\hline $\begin{array}{l}\text { Aspheric } \\
\text { parameters }\end{array}$ & A04 & A06 & A08 & A10 & Radius (mm) & $\begin{array}{l}\text { Conic } \\
\text { Constant }\end{array}$ \\
\hline $\begin{array}{l}\text { Designed } \\
\text { Fitted }\end{array}$ & $1.954 \mathrm{E}-5$ & $-1.756 \mathrm{E}-8$ & $2.597 \mathrm{E}-11$ & $-2.414 \mathrm{E}-14$ & -22.09 & -2.271 \\
\hline
\end{tabular}




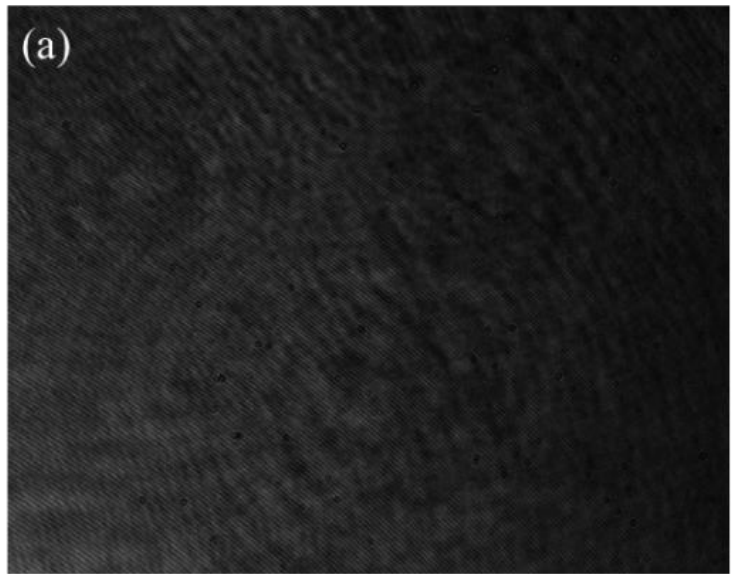

(b)

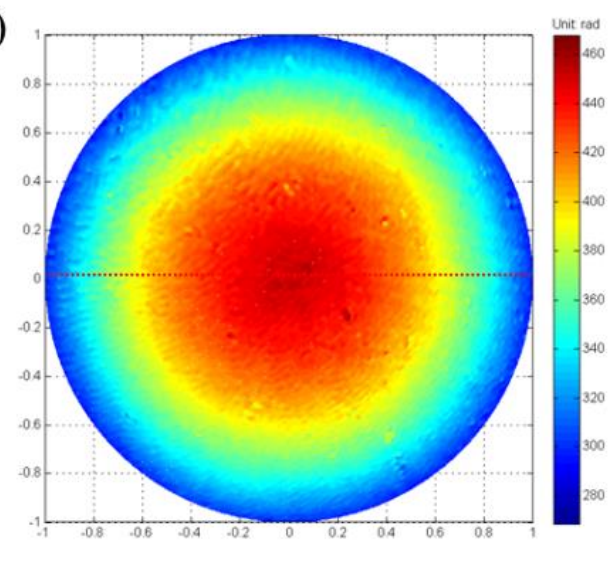

(c)

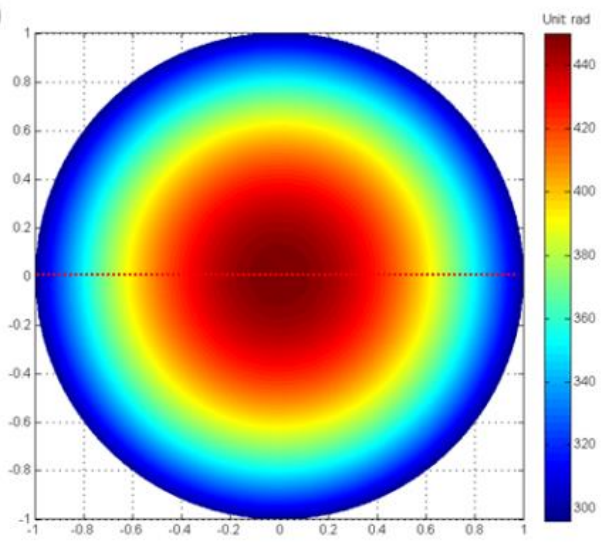

(d)

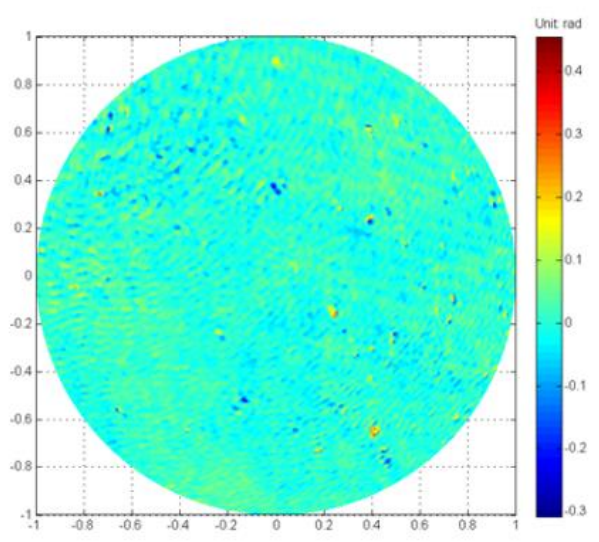

(e)

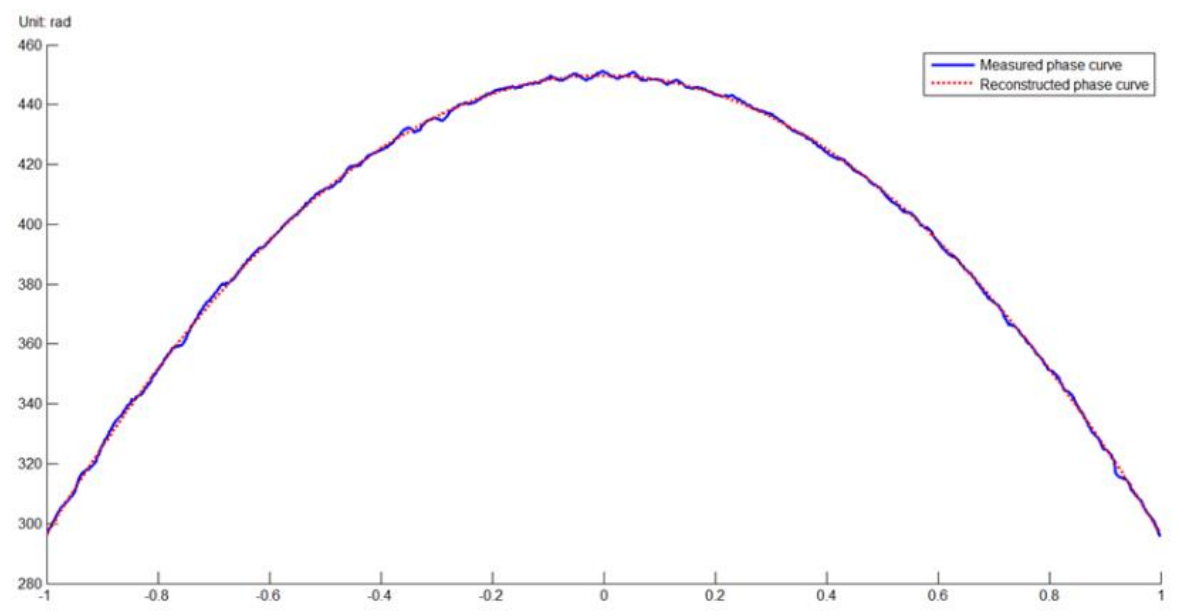

Figure 6.4 (a) Recorded digital hologram. (b) Phase reconstructed from the digital hologram (c) Reconstructed phase by fitting the phase in (b). (d) Residue phase between phase (b) and (c). (e) Profile comparison of $480^{\text {th }}$ line between designed and reconstructed curves. 


\subsection{Wavefront analysis of aspheric lens}

Zernike polynomials have been successfully used for their representation of aberrations in many different fields of optics. To know exact aberrations, Zernike coefficients must be known. They can be obtained by fitting the wavefront data with a set of polynomials by the least squares method. Where the polynomials orthogonal over the discrete unitary circle are constructed using the Gram-Schmidt method on basis of Zernike polynomials. Due to the limitation of least squares method, there will be residual coefficients in the wavefront removal of aberrations. Here, a modified method is proposed to perform the wavefront fitting.

\subsubsection{Wavefront represented by Zernike polynomials}

The wavefront can be expanded in terms of orthonormal Zernike polynomials $Z_{j}(\rho, \theta)$ in the form

$$
W(\rho, \theta)=\sum_{j} a_{j} Z_{j}(\rho, \theta)
$$

where $a_{j}$ is the Zernike coefficient. The Zernike polynomials can be written as

$$
\begin{cases}Z_{\text {even } j}(\rho, \theta)=\sqrt{2(n+1)} R_{n}^{m}(\rho) \operatorname{cosm} \theta, & m \neq 0 \\ Z_{\text {odd } j}(\rho, \theta)=\sqrt{2(n+1)} R_{n}^{m}(\rho) \sin m \theta, & m \neq 0 \\ Z_{j}(\rho, \theta)=\sqrt{n+1} R_{n}^{0}(\rho), & m=0\end{cases}
$$

where index $n$ represents the order of the polynomial, $m$ is called the azimuthal frequency. $R_{n}^{m}(\rho)$ is the radial polynomial and

$$
R_{n}^{m}(\rho)=\sum_{s=0}^{(n-m) / 2} \frac{(-1)^{s}(n-s) !}{s !\left(\frac{n+s}{2}-s\right) !\left(\frac{n-s}{2}-s\right) !} \rho^{n-2 s}
$$


The index $j$ is a polynomial-ordering number and has relationship with $n$ and $m$ in the form

$$
\begin{gathered}
n=\left[(2 j-1)^{0.5}+0.5\right]_{\text {integer }}-1 \\
m=\left\{\begin{array}{c}
2\{[2 j+1-n(n+1)] / 4\}_{\text {integer }}, \text { when } n \text { is even } \\
2\{[2(j+1)-n(n+1)] / 4\}_{\text {integer }}-1, \text { when } n \text { is odd }
\end{array}\right.
\end{gathered}
$$

The Zernike polynomials and the relationship among $j, n$, and $m$ are given in table 6.3.

\begin{tabular}{|c|c|c|c|c|}
\hline $\bar{j}$ & $n$ & $m$ & Zernike polynomials & Aberration name \\
\hline 1 & 0 & 0 & 1 & Piston \\
\hline 2 & 1 & 1 & $\rho \cos \theta$ & $x$ tilt \\
\hline 3 & 1 & 1 & $\rho \sin \theta$ & $y$ tilt \\
\hline 4 & 2 & 0 & $\sqrt{3}\left(2 \rho^{2}-1\right)$ & Defocus \\
\hline 5 & 2 & 2 & $\sqrt{6} \rho^{2} \sin 2 \theta$ & Primary astigmatism at $45^{\circ}$ \\
\hline 6 & 2 & 2 & $\sqrt{6} \rho^{2} \cos 2 \theta$ & Primary astigmatism at $\mathrm{O}^{\circ}$ \\
\hline 7 & 3 & 1 & $\sqrt{8}\left(3 \rho^{3}-2 \rho\right) \sin \theta$ & Primary y coma \\
\hline 8 & 3 & 1 & $\sqrt{8}\left(3 \rho^{3}-2 \rho\right) \cos \theta$ & Primary $x$ coma \\
\hline 9 & 3 & 3 & $\sqrt{8} \rho^{3} \sin 3 \theta$ & \\
\hline 10 & 3 & 3 & $\sqrt{8} \rho^{3} \cos 3 \theta$ & \\
\hline 11 & 4 & 0 & $\sqrt{5}\left(6 \rho^{4}-6 \rho^{2}+1\right)$ & Primary spherical \\
\hline 12 & 4 & 2 & $\sqrt{10}\left(4 \rho^{4}-3 \rho^{2}\right) \cos 2 \theta$ & Secondary astigmatism at $\mathrm{O}^{\circ}$ \\
\hline 13 & 4 & 2 & $\sqrt{10}\left(4 \rho^{4}-3 \rho^{2}\right) \sin 2 \theta$ & Secondary astigmatism at $45^{\circ}$ \\
\hline 14 & 4 & 4 & $\sqrt{10} \rho^{4} \cos 4 \theta$ & \\
\hline 15 & 4 & 4 & $\sqrt{10} \rho^{4} \sin 4 \theta$ & \\
\hline
\end{tabular}

Table 6.3. Zernike polynomials [106].

In order to demonstrate above Zernike polynomials more visually, Fig. 6.5 shows each term by indexing order in table 6.3. It can be seen that each term has unique aberration shape with respect its physical meaning. 


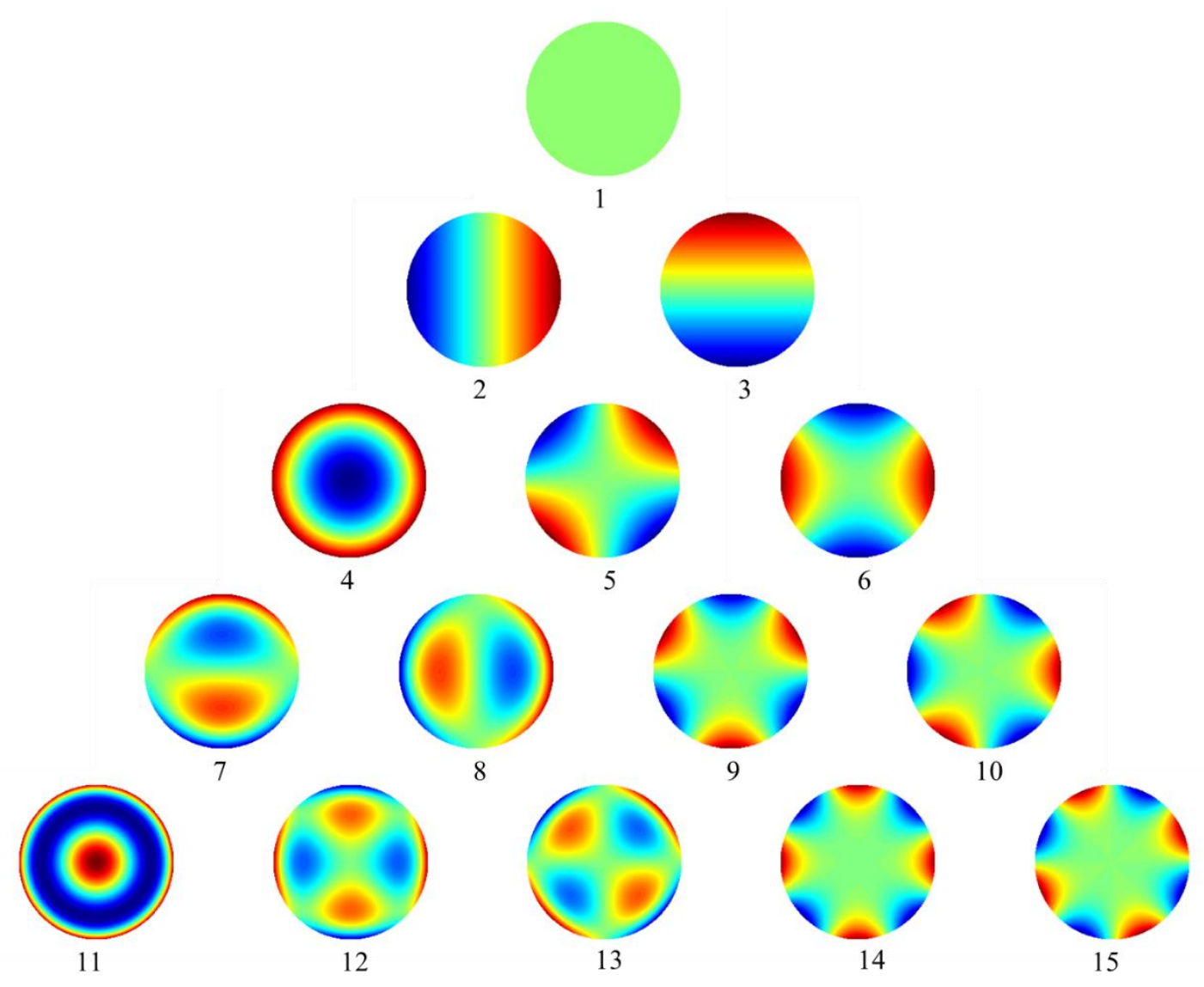

Figure 6.5 Zernike polynomials display based on indexing order.

\subsubsection{Aperture apodization}

Zernike polynomials are defined in polar coordinate system. They are orthogonal on continuous unitary circle. In data fitting, in order to make use this orthogonality feature, the wavefront for evaluation should be apodized in polar coordinate and normalized into a unit circle. In polar coordinate, the wavefront data are coordinated by polar angle and polar radius. Therefore, the wavefront is usually presented by the form as displayed in Fig. 6.6. 


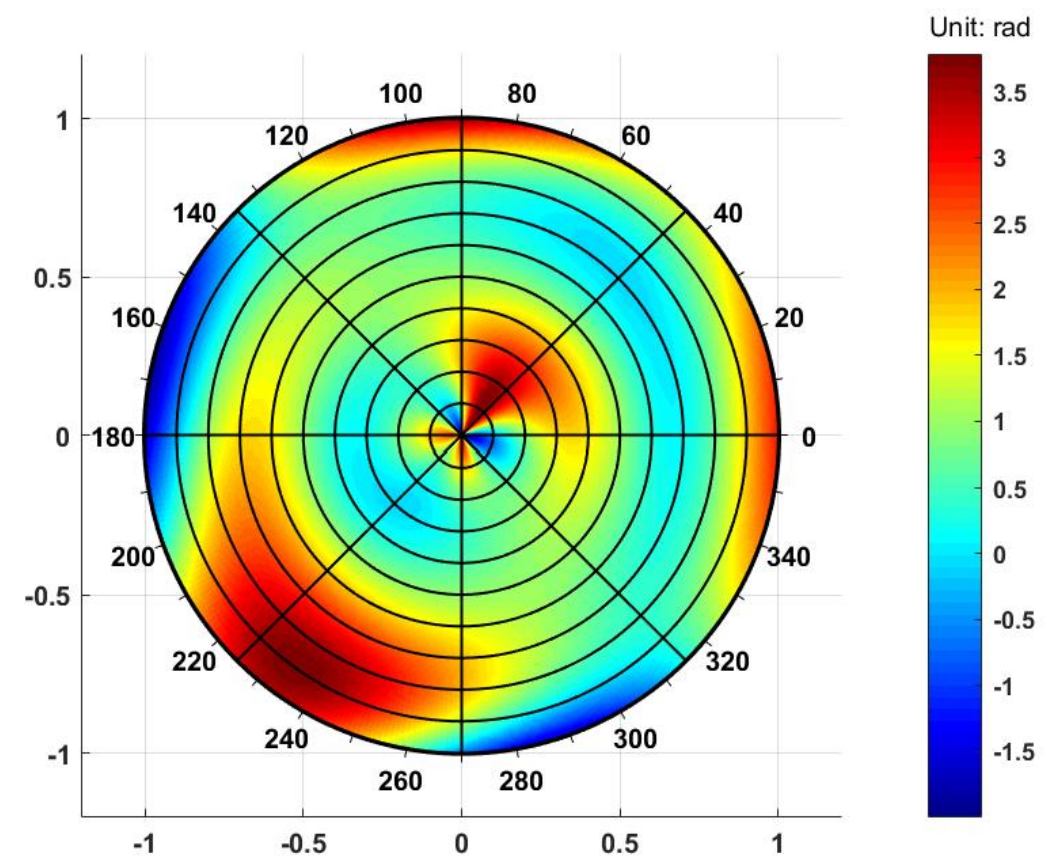

Figure 6.5 Wavefront in Polar coordinate.

In fact, although the orthogonality in polar coordinate system is so important, one also needs to face a very common situation - the wavefront data is often in the form of a discrete matrix which keeps consistent with the data from an imaging sensor. As shown in Fig. 6.7, the wavefront is displayed in Cartesian coordinate system. This brings gap between Zernike polynomials orthogonality in polar coordinate system and the wavefront data in discrete Cartesian coordinate system. Necessary conversion must be done to finish implement the fitting.

Two ways can solve above problem. One way is to map the wavefront data into Polar coordinate system from Cartesian coordinate system. This action will lost data precision and accuracy with no doubt due to interpolation. The other way is to convert Zernike polynomials into discrete polynomials by Gram-Schmidt orthogonalization which are orthogonal in Cartesian coordinate system. This method keeps polynomials orthogonality 
and provides flexibility for discrete data fitting. In this research, we prefer to the latter method for wavefront evaluation.

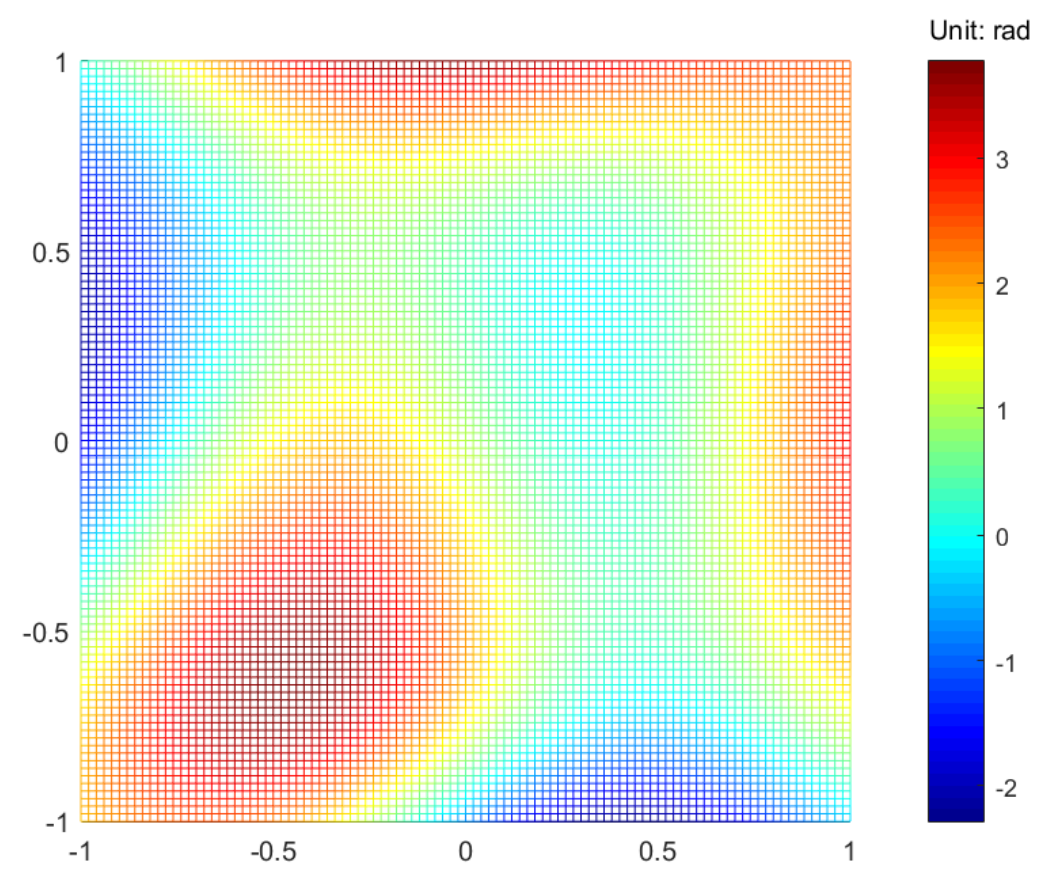

Figure 6.7 Wavefront in Cartisian coordinate.

Another work is still needed before fitting procedure. As we apply Cartesian coordinate system in wavefront fitting, the data needs normalized into unit circle yet. The strategy we use is as displayed in Fig. 6.8. We assume the matrix is in its circumcircle. 


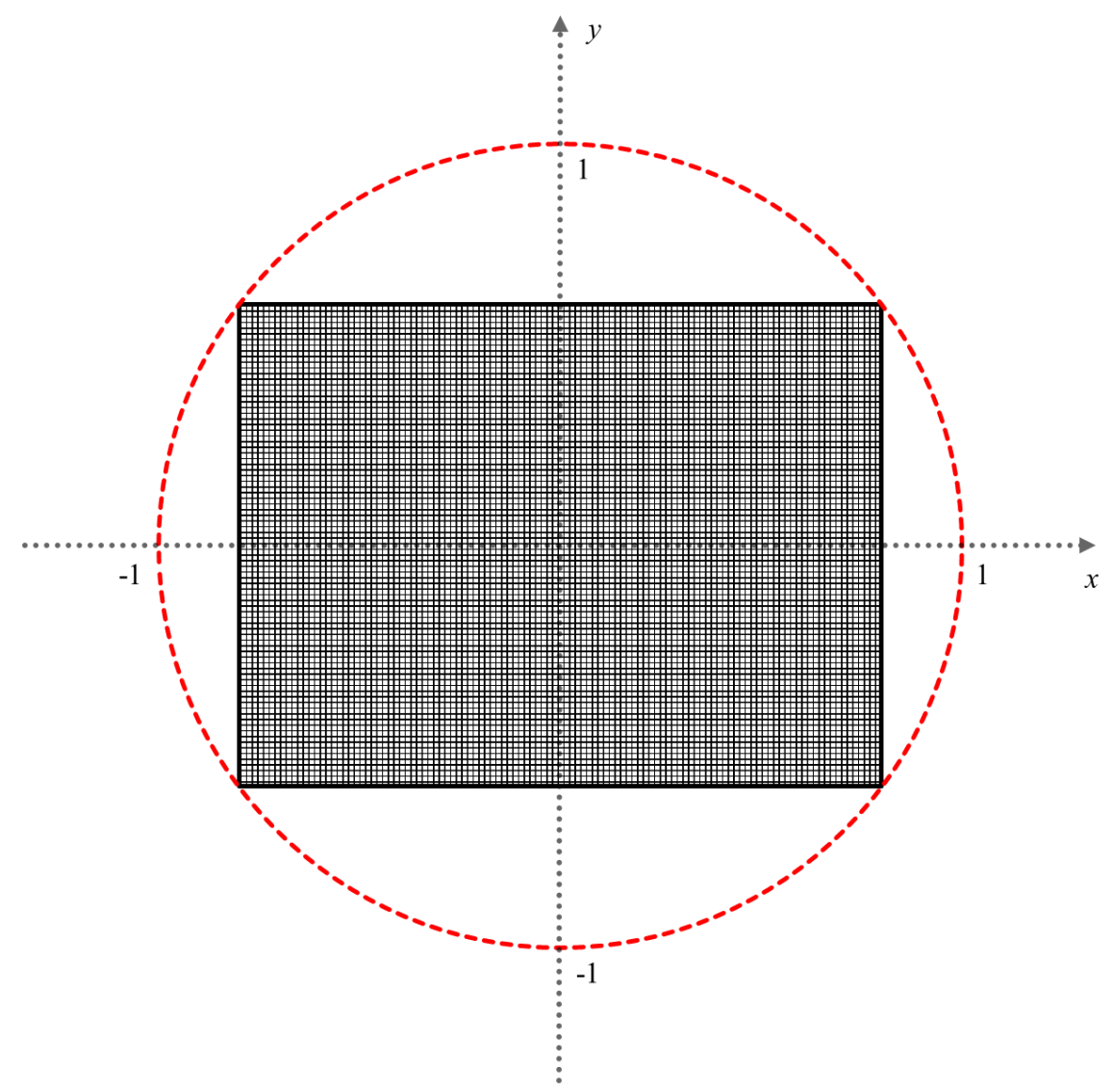

Figure 6.8 Apodozation in Cartisian coordinate.

\subsubsection{Generation orthogonal polynomials by Gram-Schmidt orthogonalization}

Zernike polynomials are orthogonal on continuous unitary circle but not on discrete unitary circle. Thus, Gram-Schmidt orthogonalization is carried out to construct a set of polynomials which are orthogonal on the discrete unitary circle

$$
\left(\begin{array}{lllll}
V_{1} & V_{2} & \ldots & V_{j-1} & V_{j}
\end{array}\right)=\left(\begin{array}{lllll}
Z_{1} & Z_{2} & \ldots & Z_{j-1} & Z_{j}
\end{array}\right)\left(\begin{array}{cccccc}
1 & 0 & \ldots & 0 & 0 \\
c_{2,1} & 1 & \ldots & 0 & 0 \\
\vdots & \vdots & \ddots & \vdots & \vdots \\
c_{j-1,1} & c_{j-1,2} & \ldots & 1 & 0 \\
c_{j, 1} & c_{j, 2} & \ldots & c_{j, j-1} & 1
\end{array}\right)
$$

Or in a general way 


$$
V_{j}=Z_{j}+\sum_{i=1}^{j-1} c_{j, i} Z_{i}
$$

On the discrete unitary circle, the polynomials $V_{j}(\rho, \theta)$ satisfy the following condition

$$
\sum_{k=1}^{N} V_{j}\left(\rho_{i}, \theta_{i}\right) V_{p}\left(\rho_{i}, \theta_{i}\right)=0
$$

Then the $c_{j, i}$ can be written

$$
c_{j, i}=\sum_{l=1}^{j-1}\left\{c_{j-l, i}\left[\left(\sum_{k=1}^{N} Z_{j}\left(\rho_{k}, \theta_{k}\right) V_{j-l}\left(\rho_{k}, \theta_{k}\right)\right) /\left(\sum_{k=1}^{N} V_{j-l}^{2}\left(\rho_{k}, \theta_{k}\right)\right)\right]\right\}
$$

Where $i=1,2,3, \ldots, j-1$ and $\mathrm{c}_{j j}=1$.

We get the wavefront expressed by orthogonal polynomials $V_{j}$

$$
W(\rho, \theta)=\sum_{j} b_{j} V_{j}(\rho, \theta)
$$

Here, we define the mean value of the wavefront as

$$
\bar{W}=\frac{1}{L} \sum_{k=1}^{N} W\left(\rho_{k}, \theta_{k}\right)=\sum_{j} b_{j} \overline{V_{j}}
$$

where $\overline{V_{j}}=\frac{1}{N} \sum_{k=1}^{N} V_{j}\left(\rho_{k}, \theta_{k}\right), L$ is the maximum value of the index $j$.

Then we reconstruct the orthogonal polynomials by subtracting the mean value of the wavefront

$$
W(\rho, \theta)-\bar{W}=\sum_{j} b_{j}\left(V_{j}(\rho, \theta)-\overline{V_{j}}\right)
$$

For the first term of the polynomials is piston terms, which mean value is a constant. So we reset the above polynomials as $\left(V_{2}-\overline{V_{2}}\right), \ldots,\left(V_{j}-\overline{V_{j}}\right)$, it is easy to verify the still orthogonal on discrete unitary circle. The Eq. 6.34 can be written as

$$
W(\rho, \theta)-\bar{W}=\sum_{j=2}^{L} b_{j}\left(V_{j}(\rho, \theta)-\overline{V_{j}}\right)
$$




\subsubsection{Least squares method}

Assume the measured wavefront data is $W\left(\rho_{k}, \theta_{k}\right)$, and the deviation of fitting is $\delta^{2}$.The wavefront is then fitted by orthogonal polynomials with least square method.

$$
\delta^{2}=\sum_{k=1}^{N}\left(W\left(\rho_{k}, \theta_{k}\right)-\bar{W}-\sum_{j=2}^{L} b_{j}\left(V_{j}\left(\rho_{k}, \theta_{k}\right)-\bar{V}_{j}\left(\rho_{k}, \theta_{k}\right)\right)\right)^{2}
$$

In order to make the $\delta^{2}$ as small as possible, differential is taken on the both side of the Eq. 6.36

$$
\frac{\partial \delta^{2}}{\partial b_{j}}=\frac{\partial \sum_{k=1}^{N}\left(W\left(\rho_{k}, \theta_{k}\right)-\bar{W}-\sum_{j=2}^{L} b_{j}\left(V_{j}\left(\rho_{k}, \theta_{k}\right)-\bar{V}_{j}\left(\rho_{k}, \theta_{k}\right)\right)\right)^{2}}{\partial b_{j}}=0
$$

Thus, coefficients $b_{j}$ can be obtained by solving the equation.

$$
b_{j}=\frac{\sum_{k=1}^{N}\left(W\left(\rho_{k}, \theta_{k}\right)-\bar{W}\right)\left(V_{j}\left(\rho_{k}, \theta_{k}\right)-\bar{V}_{j}\left(\rho_{k}, \theta_{k}\right)\right)}{\sum_{k=1}^{N}\left(V_{j}\left(\rho_{k}, \theta_{k}\right)-\overline{V_{j}}\left(\rho_{k}, \theta_{k}\right)\right)^{2} ?}
$$

Where $j=2,3, \ldots L$.

The first piston term $V_{1}$ is composed of $W-\bar{W}-\sum_{j=2}^{L} b_{j}\left(V_{j}-\overline{V_{j}}\right), \bar{W}$ and $\sum_{j=2}^{L} b_{j} \bar{V}_{j}$, so the coefficient of the piston term can be written as

$$
b_{1}=\bar{W}-\sum_{j=2}^{L} b_{j} \bar{V}_{j}+\frac{\sum_{k=1}^{N}\left(W\left(\rho_{k}, \theta_{k}\right)-\bar{W}-\sum_{j=2}^{L} b_{j}\left(\rho_{k}, \theta_{k}\right)\left(V_{j}\left(\rho_{k}, \theta_{k}\right)-\overline{V_{j}}\right)\right) V_{1}\left(\rho_{k}, \theta_{k}\right)}{\sum_{k=1}^{N}\left(V_{1}\left(\rho_{k}, \theta_{k}\right)\right)^{2}}
$$

\subsubsection{Zernike coefficients}

Since we know the coefficients $b_{j}$ and $c_{j i}$ the Zernike coefficients $a_{j}$ can be got by substituting the Eqs. 6.34 and 6.37 into the Eq. 6.29 . 


$$
W=b_{1} Z_{1}+\sum_{j=2}^{L} b_{j}\left(Z_{j}+\sum_{i=1}^{j-1} c_{j i} Z_{i}\right)
$$

Comparing with Eq. 6.28, we can get the coefficients $a_{j}$

$$
a_{j}=b_{j}+\sum_{i=j+1}^{L} b_{i} c_{i j}
$$

Where $j=1,2,3, \ldots,(L-1)$ and $a_{L}=b_{L}$.

\subsubsection{Simulation}

In order to test the proposed method, simulation with different levels of Gaussian noise has been done. In this simulation, 15 terms of Zernike polynomials are employed to generate a wavefront for fitting. While their coefficients are generated by Rand function in MATLAB which locate at $[-0.5,0.5]$. The coefficients distribution is shown in Fig. 6.9.

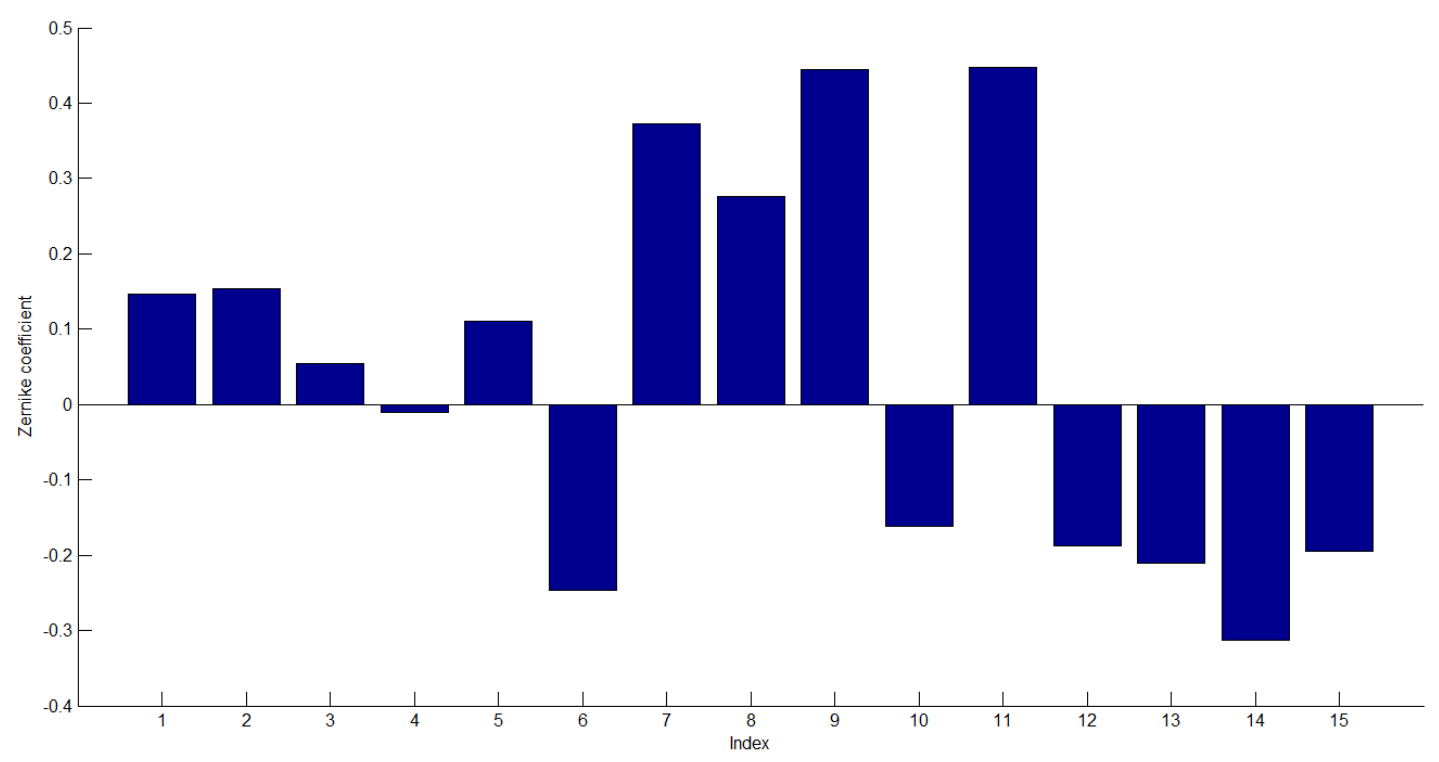

Figure 6.9 Random Zernike coefficients distribution.

The wavefront is the summation of all the Zernike polynomials multiplying their coefficients. The wavefront is generated in a unit circle. To map it onto Cartesian coordinate, a matrix with $500 \times 500$ is used. The apodization and normalization are applied 
based on the matrix size. The range for both $x$ - and $y$-axes are $[-0.707,0.707]$. The wavefront is rendered with jet colormap and shown in Fig. 6.10(a). Its 3D rendering is displayed in Fig. 6.10(b).

(a)

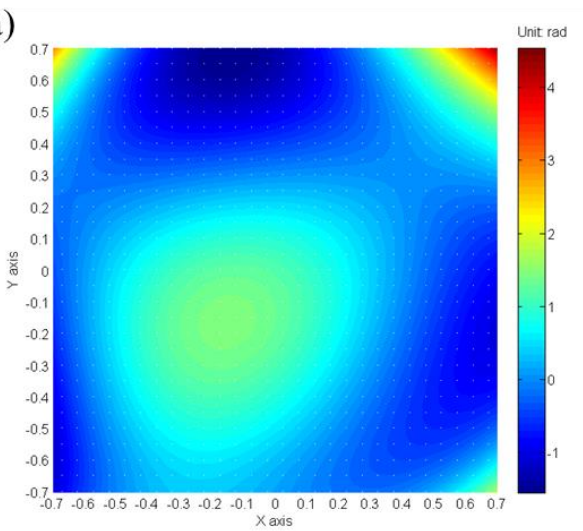

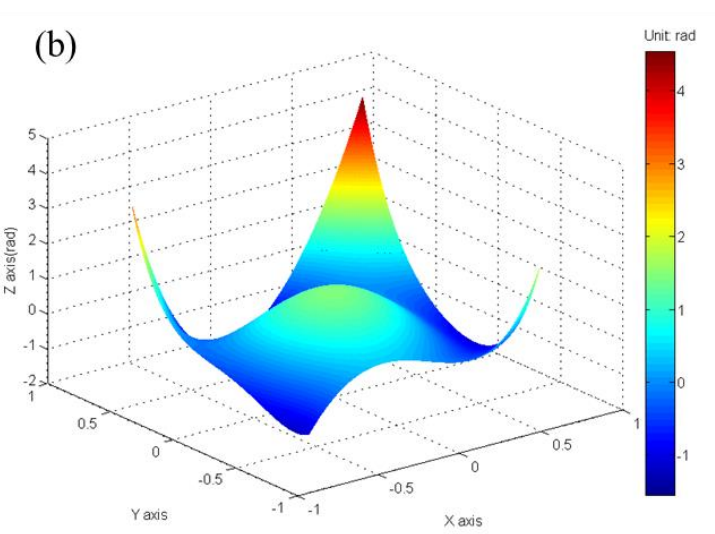

Figure 6.10 (a) simulated wavefront, and its (b) 3D rendering.

In fitting, we use 5 loops for iteration to retrieve the correct coefficients. In table 6.4, we list the simulated coefficients and fitted ones. It can be seen that with the same iteration loops when the noise increases, the fitting accuracy will go down. Of course, we can increase iteration loops to improve the fitting results, but also have to spend more running time. In order to demonstrate the fitting results more visually, figure 6.11 shows the reconstructed wavefronts and theirs residues with respect to the simulated wavefront. We can see that in Fig. 6.11(h), the variance has corresponded to $1 / 4$ of the simulated wavefront PV values. In fact, the systematic noise is impossible to reach to so high level. By doing so, we show that our method is robust in presence of noise. 
Table 6.4 Fitting results of simulated wavefront.

\begin{tabular}{llllll}
\hline Index & \multicolumn{1}{c}{$\begin{array}{c}\text { Simulated } \\
\text { value }\end{array}$} & $\begin{array}{c}\text { Fitted value } \\
(\text { variance }=0)\end{array}$ & $\begin{array}{c}\text { Fitted value } \\
\left(\text { variance }=10^{-5}\right)\end{array}$ & $\begin{array}{c}\text { Fitted value } \\
\left(\text { variance }=10^{-4}\right)\end{array}$ & $\begin{array}{c}\text { Fitted value } \\
\left(\text { variance }=10^{-3}\right)\end{array}$ \\
\hline 1 & 0.146 & 0.1460 & 0.1460 & 0.1468 & 0.1516 \\
2 & 0.154 & 0.1540 & 0.1540 & 0.1545 & 0.1542 \\
3 & 0.055 & 0.0550 & 0.0550 & 0.0558 & 0.0594 \\
4 & -0.011 & -0.0110 & -0.0110 & -0.0102 & -0.0055 \\
5 & 0.110 & 0.1100 & 0.1101 & 0.1098 & 0.1100 \\
6 & -0.247 & -0.2470 & -0.2472 & -0.2480 & -0.2519 \\
7 & 0.373 & 0.3730 & 0.3730 & 0.3734 & 0.3744 \\
8 & 0.276 & 0.2760 & 0.2760 & 0.2762 & 0.2764 \\
9 & 0.445 & 0.4450 & 0.4450 & 0.4444 & 0.4398 \\
10 & -0.161 & -0.1610 & -0.1611 & -0.1607 & -0.1590 \\
11 & 0.448 & 0.4480 & 0.4481 & 0.4483 & 0.4500 \\
12 & -0.188 & -0.1880 & -0.1881 & -0.1883 & -0.1898 \\
13 & -0.210 & -0.2100 & -0.2099 & -0.2100 & -0.2095 \\
14 & -0.313 & -0.3130 & -0.3131 & -0.3125 & -0.3072 \\
15 & -0.195 & -0.1950 & -0.1951 & -0.1949 & -0.1931 \\
\hline
\end{tabular}


(a)

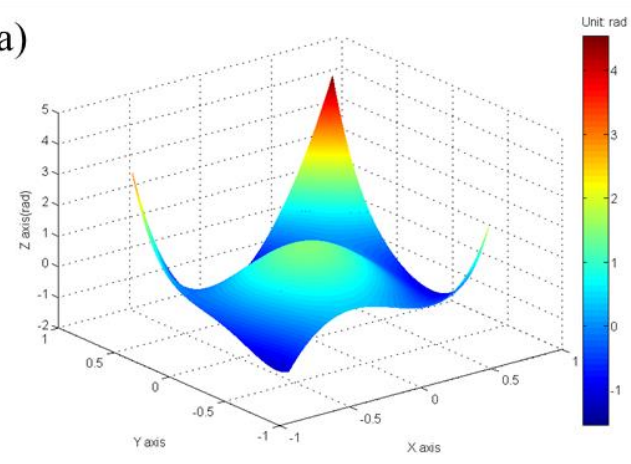

(c)

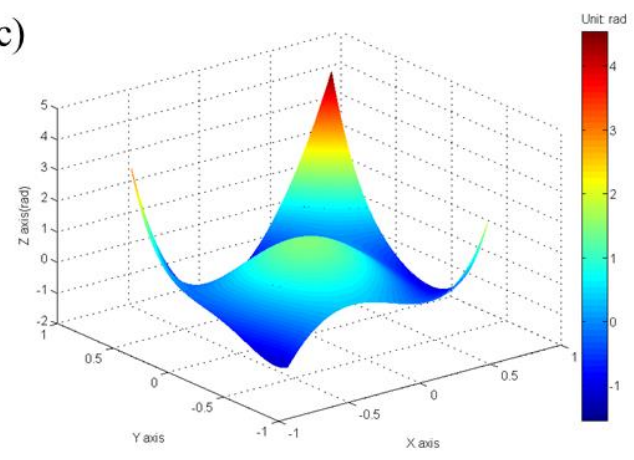

(e)

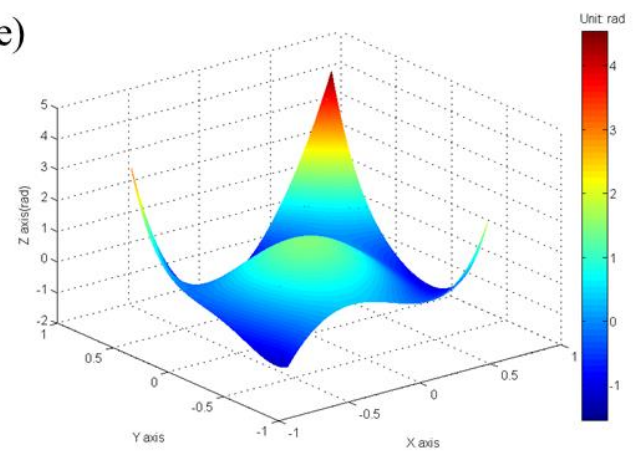

(g)

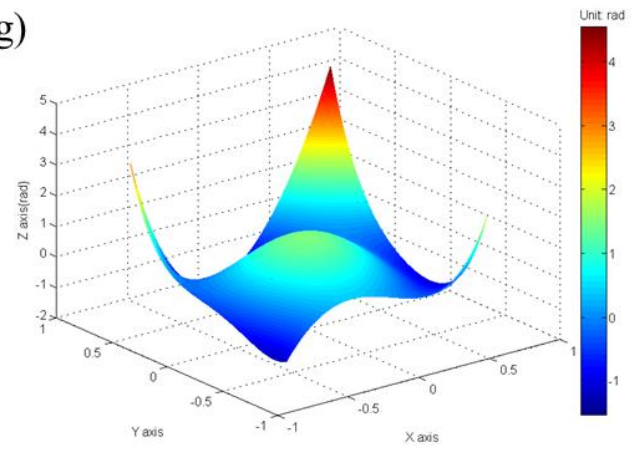

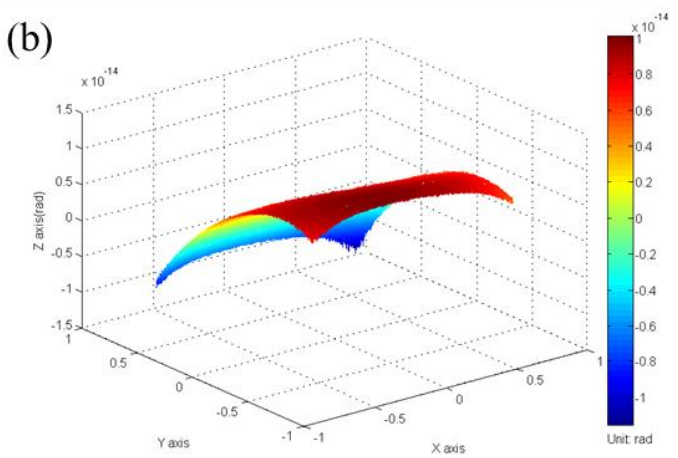

(d)

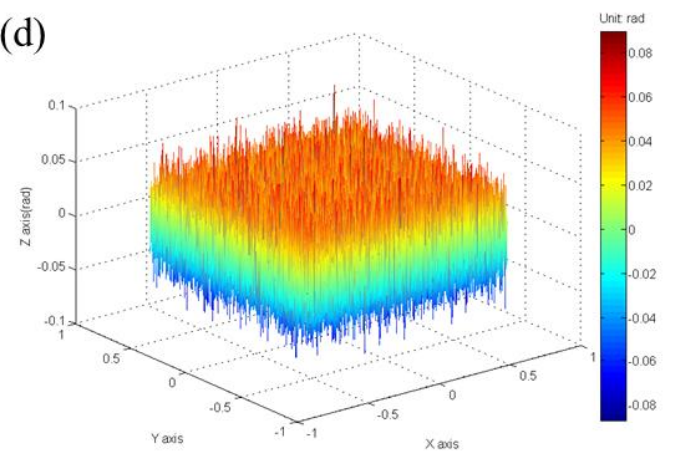

(f)

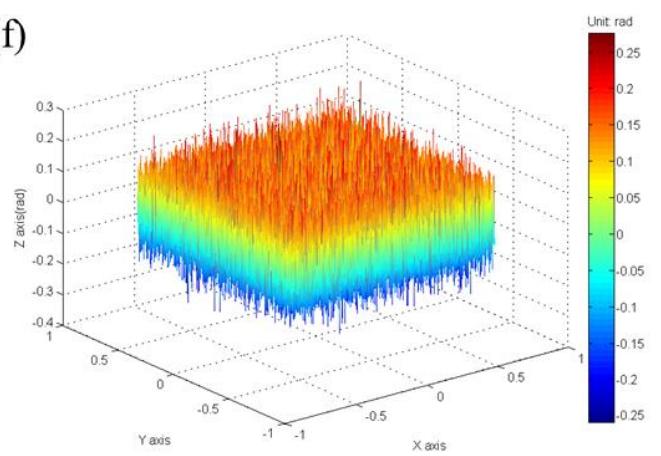

(h)

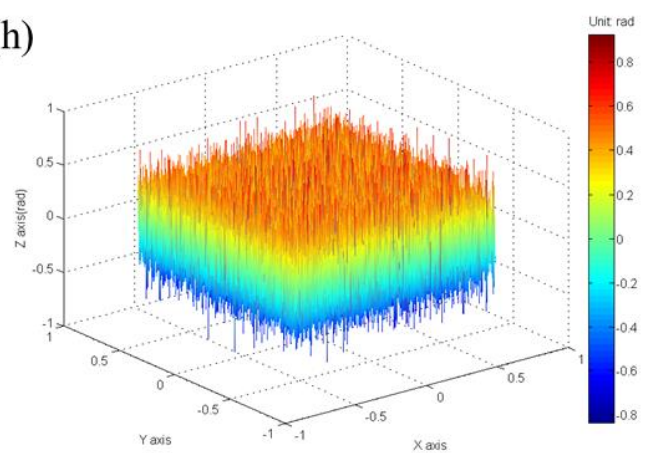

Figure 6.11 (a) Reconstructed wavefront when noise variance $=0$, and its (b) residue, (c) reconstructed wavefront when noise variance $=10^{-5}$, and its (d) residue, (e) reconstructed wavefront when noise variance $=10^{-4}$, and its (f) residue, $(\mathrm{g})$ reconstructed wavefront when noise variance $=10^{-3}$, and its $(\mathrm{h})$ residue. 
Figures 6.12-6.14 show PV value, mean value and standard deviation curves computed from residues. The PV value increases with the variance increasing. While the mean value is very close to zero which coincides with the mean value set in simulation (mean value $=0$ ). The standard deviation value also increases with the variance increasing. This agrees with the trend of variation in the residues in Fig. 6.11.

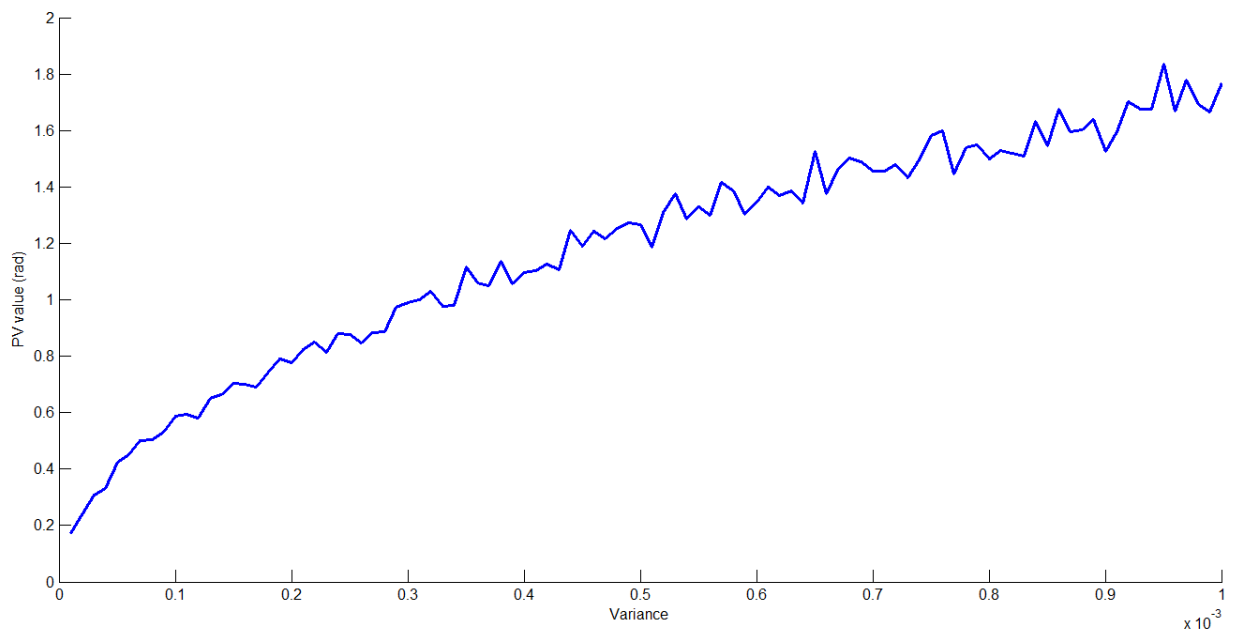

Figure 6.12 PV value curve.

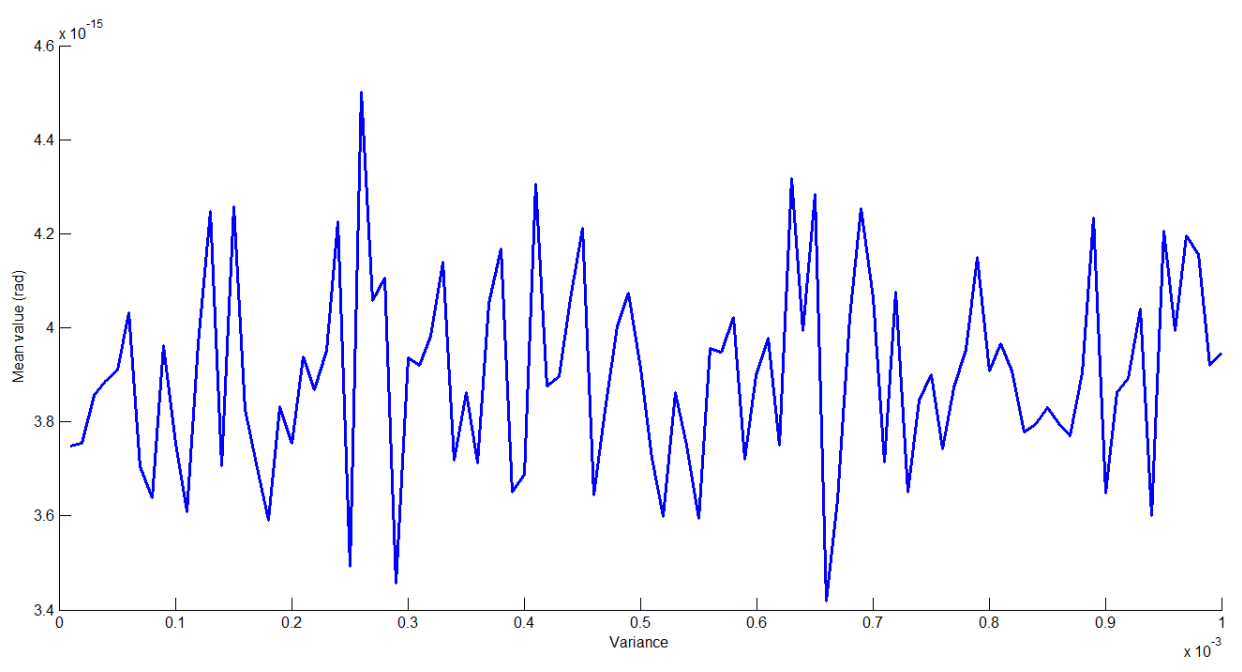

Figure 6.13 Mean value curve. 


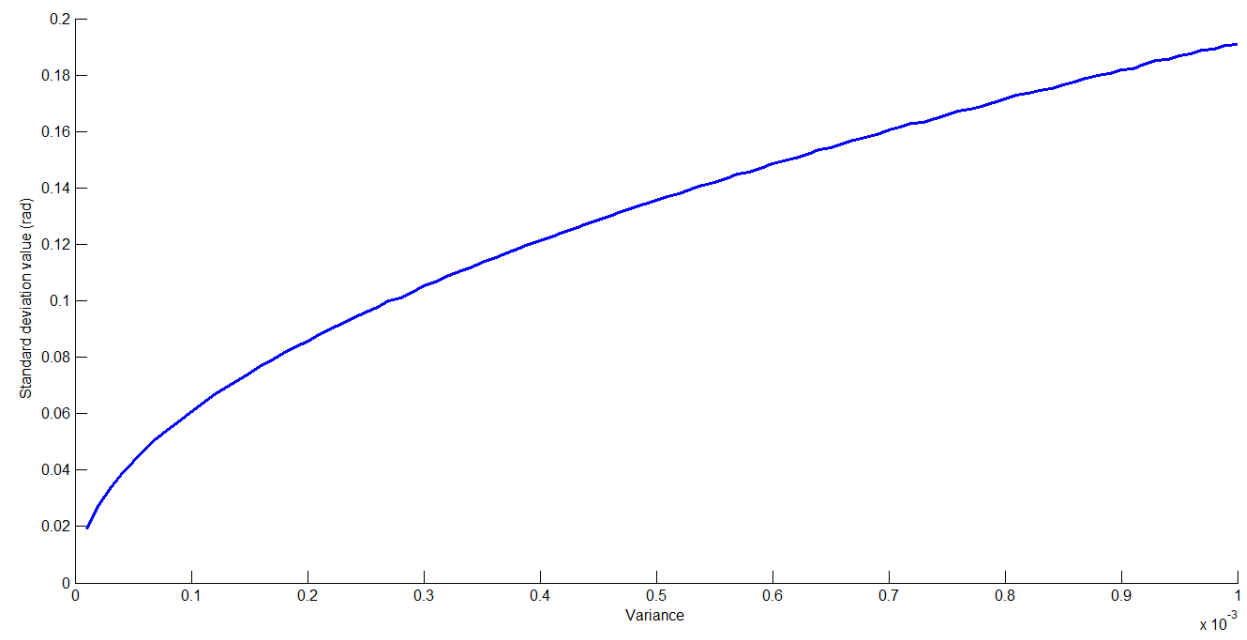

Figure 6.14 Standard deviation curve.

\subsubsection{Experimental testing}

In the experiment, a high-precision aspheric lens (model: KPA16) from Newport is used for testing. The diameter of the aspheric lens is $25 \mathrm{~mm}$ and the focal length is $37.5 \mathrm{~mm}$. In order to investigate whether the aspheric lens improves imaging performance, a simulation in Zemax 2015 is done. In Fig. 6.15, the lens editor shows the setting for the aspheric lens. Its layout is displayed in Fig. 6.16. To evaluate its performance, we place the image plane at its focal plane $(37.5 \mathrm{~mm}$ from the surface 3$)$.

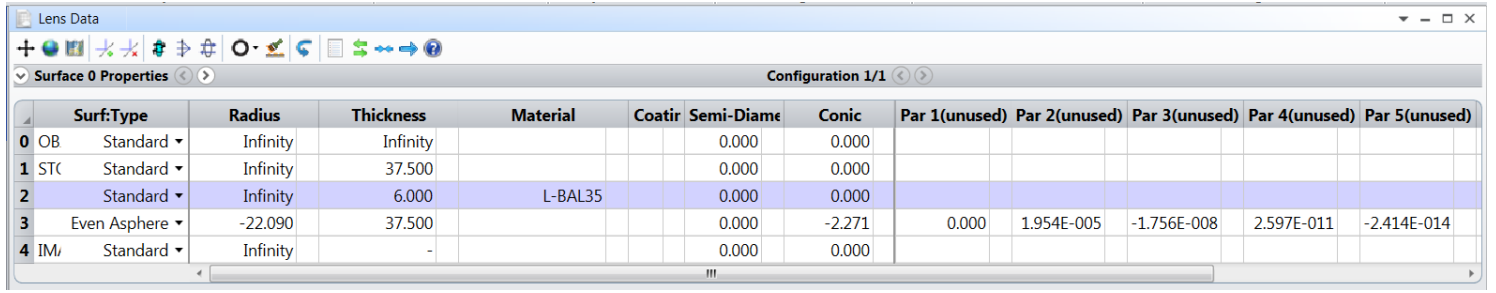

Figure 6.15 Aspheric lens editor in Zemax 2015. 


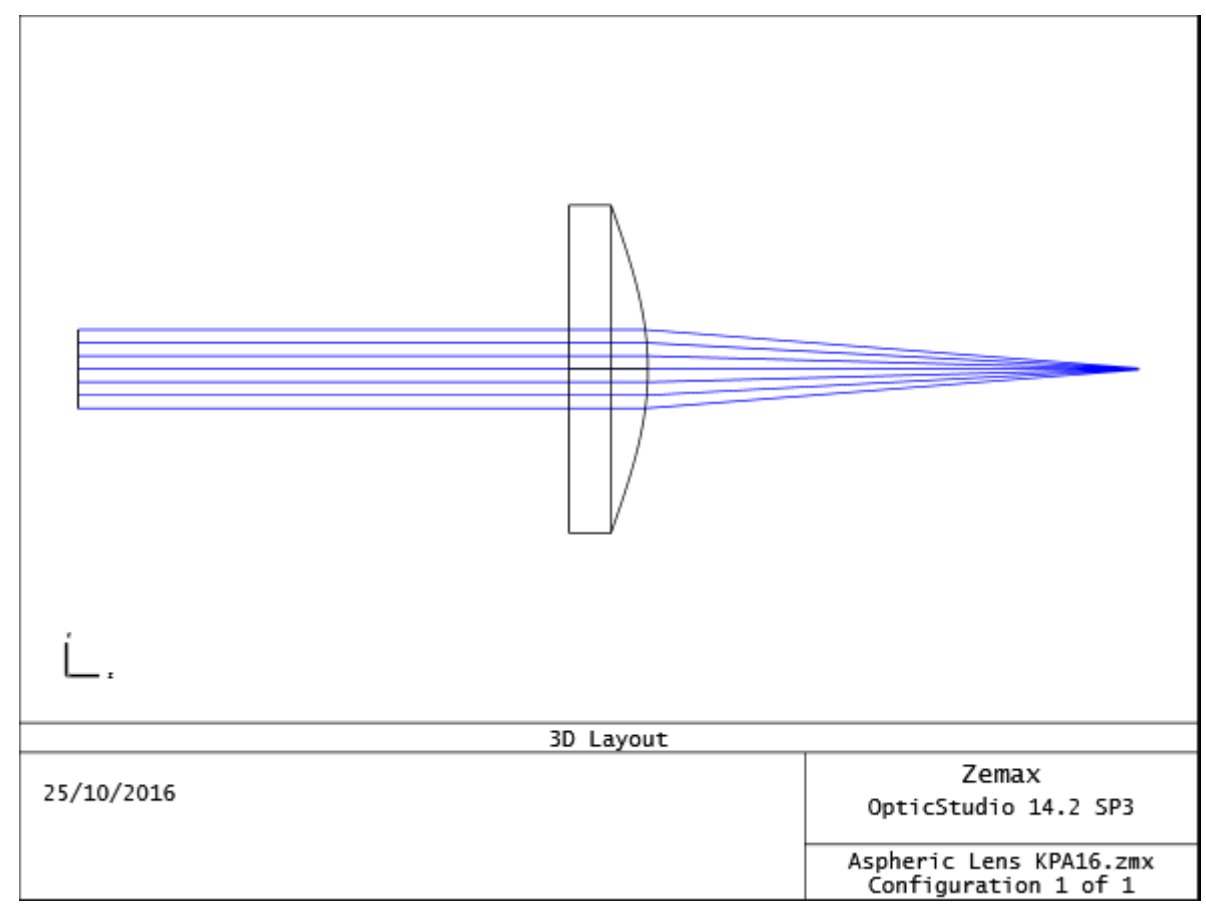

Figure 6.16 3D layout of the aspheric lens in Zemax 2015.

To compare the aspheric surface, we also remove the even order term in the definition. The aspheric lens then turns to a spherical lens. Aberrations at focal plane are computed and displayed in Figs. 6.17-6.18. From Fig. 6.17, we can see that aspheric lens mainly has spherical and defocus aberrations. While in Fig. 6.18, the spherical lens has spherical aberration. The spherical aberration in the spherical lens is more serious than that in the aspheric lens. It is notable that the high order even terms improve the aspheric lens performance in spherical aberration in particular. 


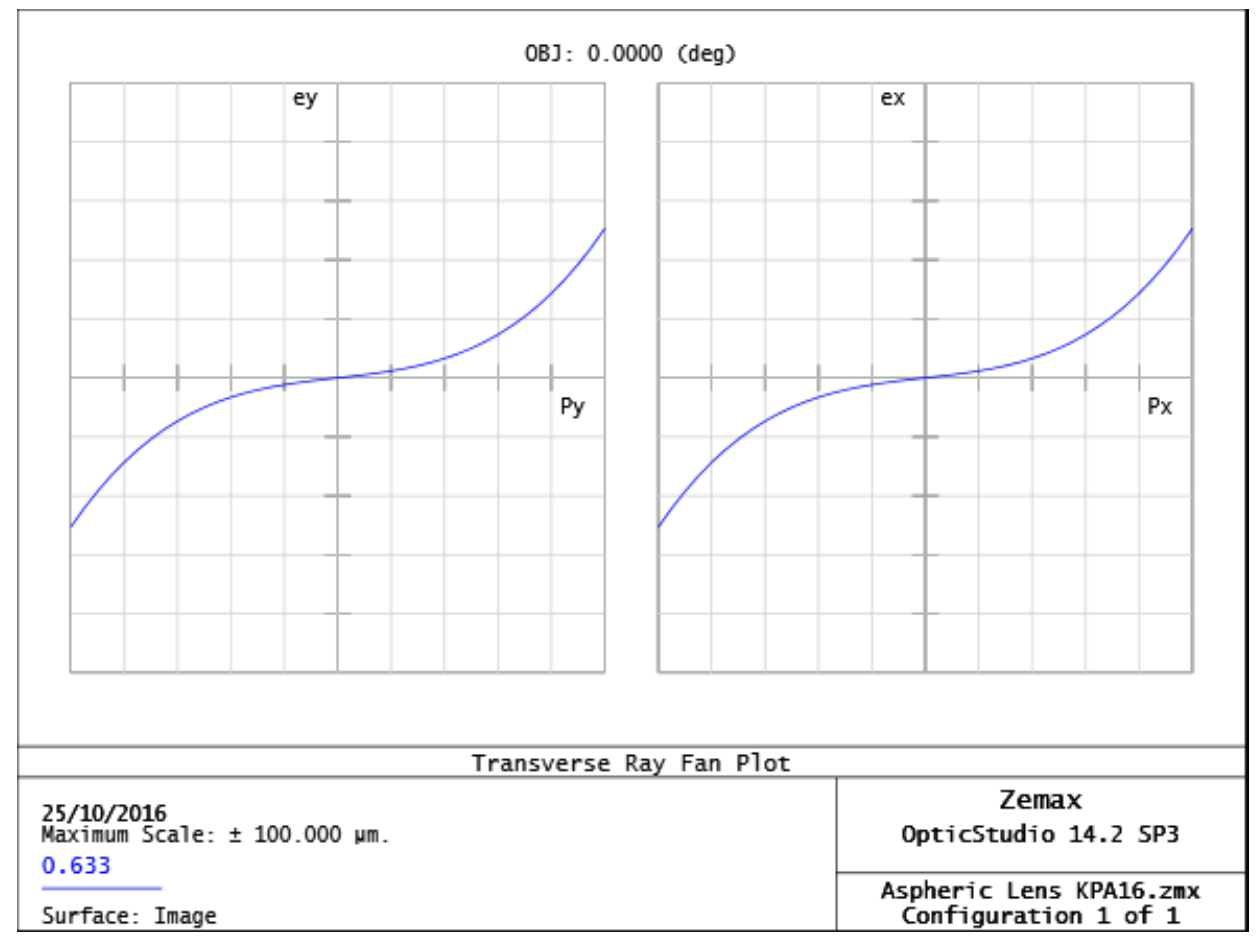

Figure 6.17 Aberrations of aspheric lens at focal plane.

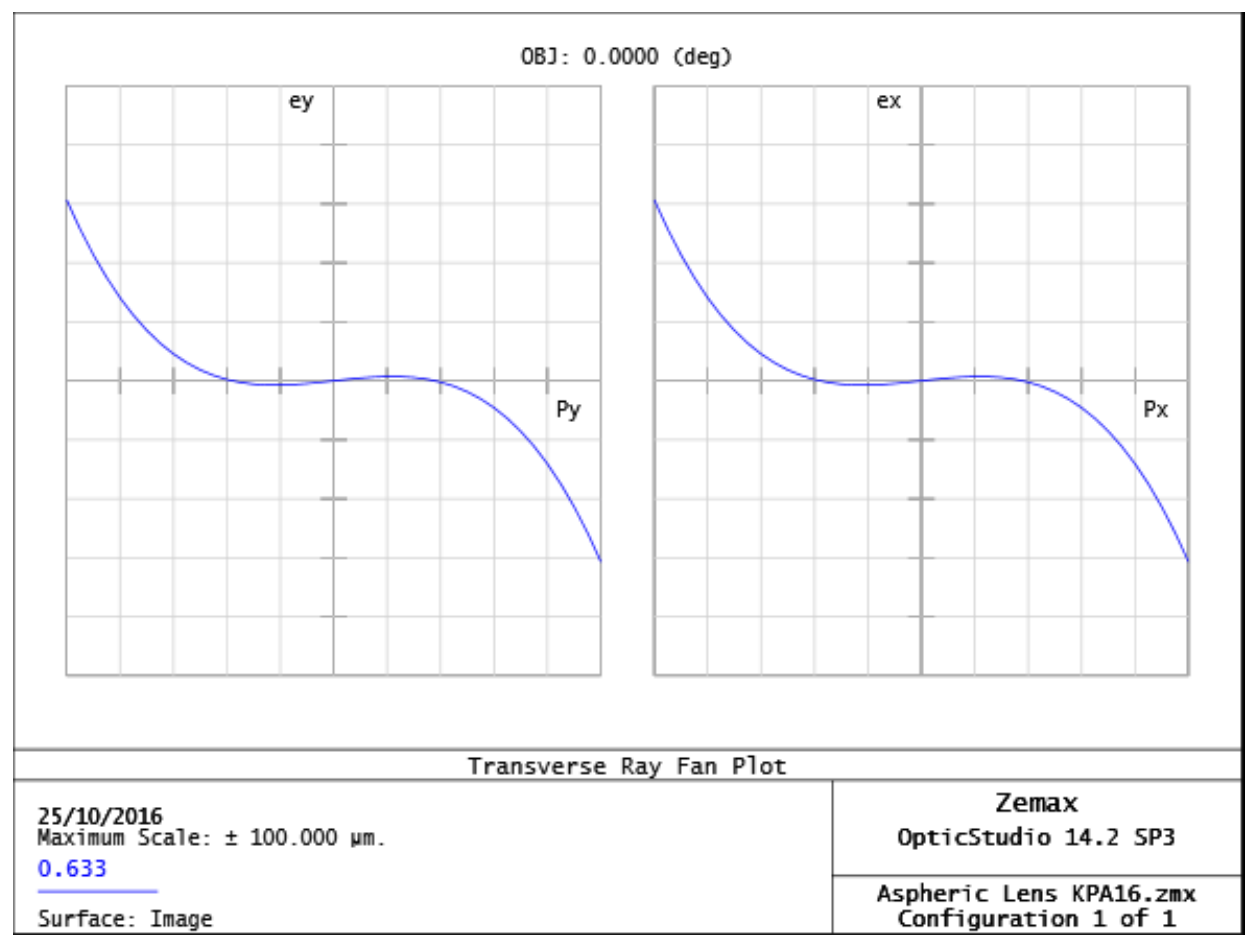

Figure 6.18 Aberrations of spherical lens at focal plane. 
To further demonstrate the proposed fitting method, an experiment is done to capture the wavefront. Figure 6.19 shows the experimental arrangement of Mach-Zehnder interferometer. Light from the laser source is spatial filtered and collimated to form a planar beam. The beam is split by BS1 into two beams: one beam is transmitted through the aspheric lens and imaged by a triplet subsequently, which forms the object beam; the other beam serves as the reference beam. The two beams then meet and interfere at BS4 with a small angular offset between them. A CCD camera is used to record the interference image-digital hologram.

In the experiment, a $660 \mathrm{~nm}$ solid laser was used as light source. The triplet is mounted Hastings achromatic triplet from Thorlabs, and its model is TRH254-040-A-ML. An 8-bit Imaging Source USB monochromatic CCD camera was used to record the digital hologram. Its resolution is $1280 \times 960$ pixels, with pixel size $4.65 \mu \mathrm{m} \times 4.65 \mu \mathrm{m}$. The distance between the aspheric lens and the triplet is $70 \mathrm{~mm}$, and that of triplet to CCD camera is $480 \mathrm{~mm}$.

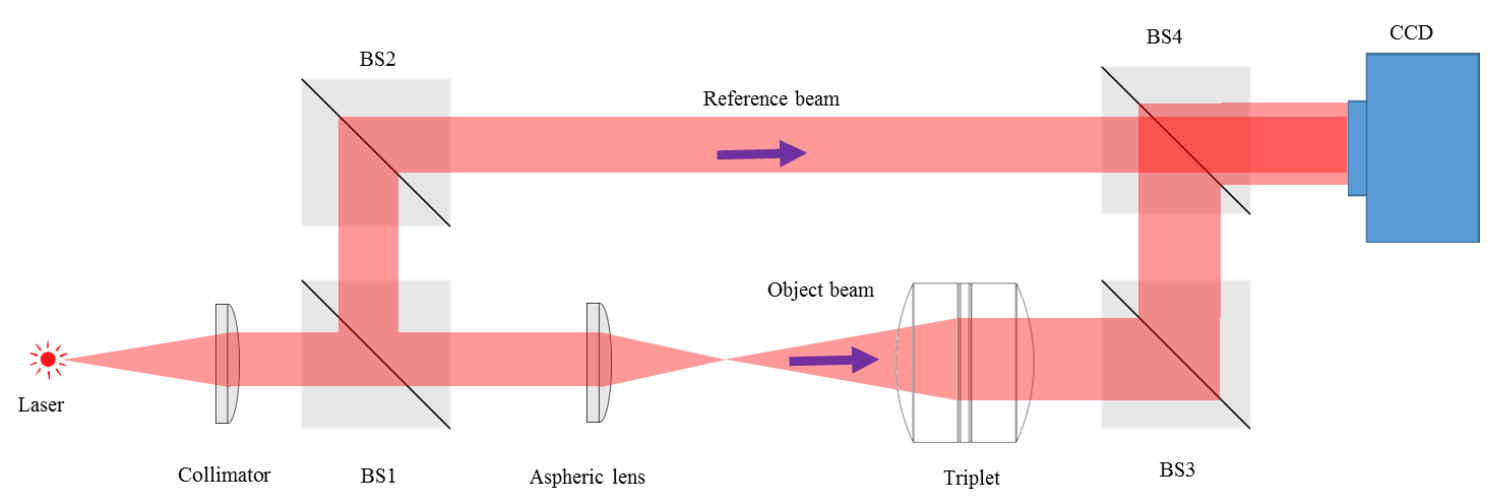

Figure 6.19 Schematic of experimental setup.

Figure 6.20(a) is the digital hologram recorded by the proposed setup, and corresponding numerical reconstruction was implemented by commercial software - Holoscope from 
d'Optron Pte Ltd. The FFT of the digital hologram is as shown in Fig. 6.20(b). It can be seen that the first order spectrum is sharp and focused which indicates the CCD camera is positioned at the image plane in the current configuration. The reconstructed wavefront is then shown in Fig. 6.20(c). Trough calibration, the magnification is 0.6. Therefore, the field of view is $7.44 \mathrm{~mm} \times 9.92 \mathrm{~mm}$. The 3D rendering of the wavefront is shown in Fig. $6.20(d)$

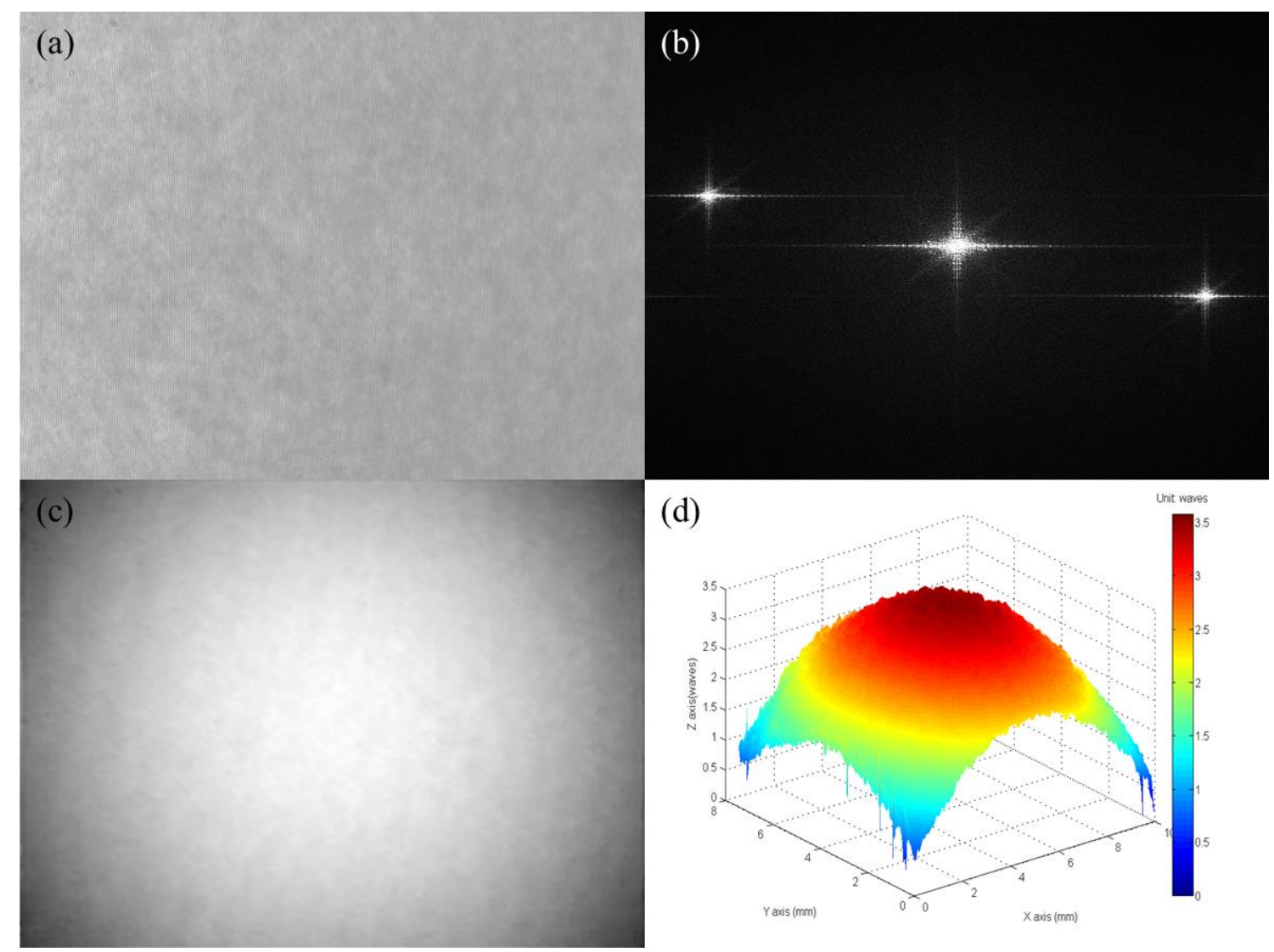

Figure 6.20 (a) Digital hologram, and its (b) Fourier transform, (c) reconstructed wavefront, and its (d) 3D rendering.

Fitting procedure is then implemented. After 5 iteration loops, the Zernike coefficients are obtained and shown in Fig. 6.21. From the figure, we can see that the main terms are the piston and spherical terms. The piston may be caused by the phase unwrapping algorithm. According to the simulation, the spherical aberration exists and this, of course, 
turns out in the measurement. The reconstructed wavefront by fitted Zernike coefficients is shown in Fig. 6.22(a). Moreover, the residue of fitting is displayed in Fig. 6.22(b). The variance in the residue is mainly caused by random noise and irregularity of optical surface in the whole system.

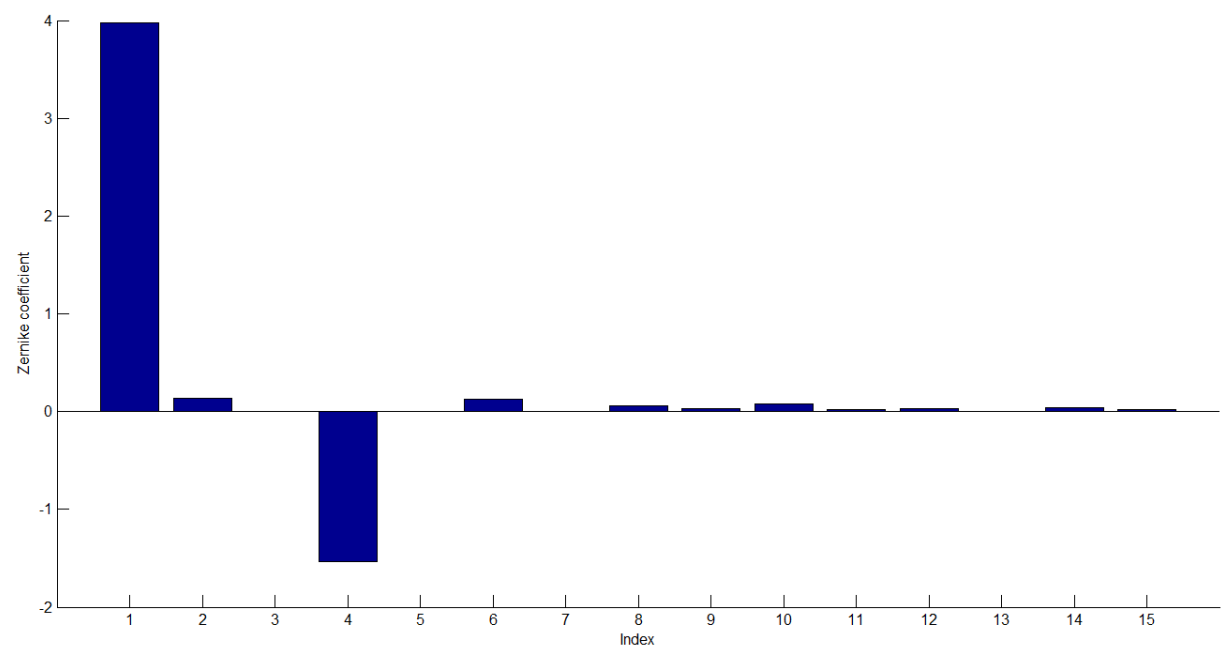

Figure 6.21 Zernike coefficiets distribution by fitting.
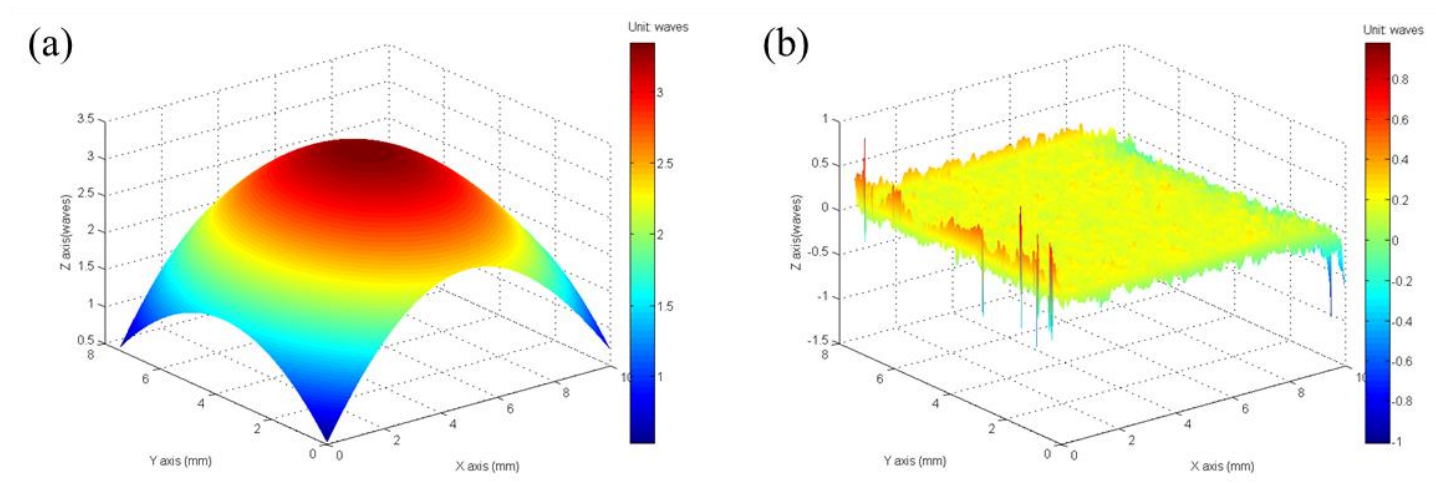

Figure 6.22 (a) Reconstructed wavefront, (b) the residue.

\subsection{Chapter summary}

In this chapter, aspheric lens characterization methods have been presented. Two aspects are investigated in characterization: aspheric parameters and wavefront Zernike coefficients. A rearranged linear expression is derived by the binomial expansion of the 
irrational term. Gram-Schmitt orthogonalization is performed on the linear polynomials. LSM is then used for fitting. The aspheric parameters are given theoretically. For the wavefront evaluation, a modified Zernike polynomials fitting method is proposed. The method is to convert the Zernike polynomials from Polar coordinate system into Cartesian coordinate system. Gram-Schmitt orthogonalization is applied on these converted polynomials. The least squares method is again used for fitting.

Simulation with different levels of noise has been done for both methods. The proposed methods are robust in presence of noise. In the experiment, the methods also work well for the aspheric lens parameters testing and the wavefront evaluation. 


\section{CONCLUSION AND FUTURE WORK}

\subsection{Conclusion}

Digital holography is a competitive 3D imaging technique for precision measurement. Since the aspheric lens is playing more and more important role in optics, its measurement and characterization are urgent in turn. This thesis studies digital holography and related techniques in measuring and characterizing the aspheric lens.

The aspheric surface definition is introduced in the beginning. The aspheric lens has so many advantages that it has been successfully applied in many fields. The aspheric surface has a more complex expression in design with respect to the spherical surface, which often leads to high gradient on the surface. To measure it in manufacturing and metrological process, many existing techniques have limitations.

A survey of current aspheric lens measurement techniques is performed. The working principles of different techniques are reviewed. Their advantages and disadvantages are discussed and concluded. The principle and three numerical reconstruction method of digital holography are introduced. Different possible configurations of digital holography are discussed according to the feature of the sample. Advantages such as quantitative phase imaging, low-cost, fast numerical reconstruction, are summarized and as a result, digital holography as a high-resolution interferometric technique has been chosen as the research tool.

Conventional aspheric lens measurement needs Null compensator, while the Null compensator for a specific aspheric lens is required to change once the aspheric lens 
parameters change. This will increase the cost and the fabrication of the Null compensator is time-consuming. We introduce a novel adaptive optical component - ELT to act as the compensator. The ETL is able to change its focal length which corresponds to different NA to match the different aspheric lens. This provides extra flexibility for the measurement. Moreover, through double-exposure, absolute phase is available which is very important for quantitative characterization.

Due to high gradients on the aspheric surface, single wavelength digital holography often fails to resolve the correct profile. The reason is analyzed based on axial resolution limitation. The solution is proposed by employing DWDH. The key technique in DWDH - dual-wavelength phase unwrapping is explored. Three existing phase unwrapping methods are discussed and modified for implementation. Moreover, a linear programming phase unwrapping method is proposed. These four methods are testing by simulation under different levels of noise. Their performances are evaluated and discussed. Different kinds of samples are tested in experiments.

Synthetic aperture digital holography is studied to solve the conflict between high lateral resolution and large FOV. Stitching is the main process in synthetic aperture digital holography. Direct alignment and feature based image stitching methods are discussed and compared. The first order spectrum based method is proposed to fast numerical reconstruction. The experiment shows the proposed method is simple and straightforward.

The aspheric lens characterization is studied in aspects of aspheric parameters and wavefront evaluation. A linear expression of the aspheric surface is derived and converted to orthogonal polynomials in Cartesian coordinate system. The aspheric 
parameters are obtained by least-squares fitting. The wavefront evaluation is performed by a modified Zernike polynomials fitting method. The method converts Zernike polynomials from Polar coordinate system into Cartesian coordinate system and orthogonalizes them. The mean value the wavefront is estimated first to accelerate the convergence speed. According to our experience, the weighted terms are also applied in first four terms.

The main contributions of this thesis are summarized as follows

- A survey and a comparison of existing aspheric lens measurement techniques

- A general introduction of digital holography

- Digital holography with ETL to measure aspheric lens

- DWDH to measure aspheric lens

- Synthetic aperture digital holography to measure aspheric lens

- Characterization of aspheric lens and its wavefront

$>$ Aspheric parameters

$>$ Zernike coefficients

\subsection{Future work}

There is still space for further enhancement and improvement. 
In chapter $4, \mathrm{DWDH}$ is able to recover much larger discontinuities than single wavelength digital holography. We mainly focus on the phase unwrapping but ignore the hardware integration and improvement. The system which can measure and reconstruct in real-time is necessary and meaningful.

In chapter 5, synthetic aperture digital holography is studied to achieve large FOV in the measurement of aspheric lens. Although this technique is simple and fast, but the reconstructed phase has discontinuities in the boundaries of the image registration. Further image processing to remove these discontinuities is meaningful. 


\section{PUBLICATION LIST}

\section{Research papers in international journals}

1. Z. Wang, W. Qu, F. Yang, and A. K. Asundi, "Focal length calibration of an electrically tunable lens by digital holography," Appl. Opt. 55, 749-756 (2016).

2. Z. Wang, W. Qu, F. Yang, A. Tian, and A. Asundi, "Absolute measurement of aspheric lens with electrically tunable lens in digital holography," Optics and Lasers in Engineering 88, 313-318 (2017).

3. Z. Wang, J. Jiao, W. Qu, F. Yang, H. Li, A. Tian, and A. Asundi, "Linear programming phase unwrapping for dual-wavelength digital holography," Appl. Opt. 56, 424-433 (2017).

4. Z. Wang, W. Qu, and A. Asundi, "A Simplified Expression for Aspheric Surface Fitting," Optik - International Journal for Light and Electron Optics, (2017).

5. Q. Weijuan, C. Oi Choo, L. Tan Rongwei, X. Qiangsheng, W. Zhaomin, X. Zhenzhong, et al., "Physical spherical phase compensation in reflection digital holographic microscopy," Optics and Lasers in Engineering 50, 563-567 (2012).

6. T. Bourgade, S. Jianfei, Z. Wang, R. Elsa, and A. Asundi, "Compact Lens-less Digital Holographic Microscope for MEMS Inspection and Characterization," e53630 (2016).

7. P. Yang, Z. Wang, Y. Yan, W. Qu, H. Zhao, A. Asundi, et al., "Close-range photogrammetry with light field camera: from disparity map to absolute distance," Appl. Opt. 55, 7477-7486 (2016).

8. J. Di, Y. Yu, Z. Wang, W. Qu, C. Y. Cheng, and J. Zhao, "Quantitative measurement of thermal lensing in diode-side-pumped Nd:YAG laser by use of digital holographic interferometry," Opt. Express 24, 28185-28193 (2016).

9. P. Yang, Z. Wang, W. Zhang, H. Zhao, W. Qu, H. Zhao, et al., " Depth profile measurement with single lenslet image by considering the positions of microlenses inside plenoptic camera," Optics and Lasers in Engineering, (under review).

\section{Research papers in international conferences}


1. Z. Wang, W. Qu, Y. Wen, F. Yang, and A. Asundi, "A new phase error compensation method in digital holographic microscopy," (2015), pp. 93023M93023M-93028.

2. Z. Wang, W. Qu, F. Yang, Y. Wen, and A. Anand, "A new autofocus method based on angular spectrum method in digital holography," (2015), pp. 94492H94492H-94497.

3. Z. Wang, W. Qu, F. Yang, Y. Wen, and A. Anand, "Digital off-axis holography with angular multiplexing and synthetic aperture," (2015), pp. 95241C-95241C95244.

4. W. Qu, Z. Wang, C. Y. Cheng, and A. Asundi, "Compound common-path digital holographic microscope," (2013), pp. 864414-864414-864416.

5. X. Peng, Y. Yu, Z. Wang, W. Qu, C. Y. Cheng, S. S. Yong, et al., "Measurement of thermal effects in solid-state laser gain medium by digital holography," (2014), pp. 895922-895922-895927.

6. W. Qu, Y. Wen, Z. Wang, F. Yang, and A. Asundi, "Capability enhancement in compact digital holographic microscopy," (2015), pp. 93020I-93020I-93028.

7. W. Qu, Y. Wen, Z. Wang, F. Yang, L. Huang, and C. Zuo, "Quantitative phase measurement for wafer-level optics," (2015), pp. 95242J-95242J-95212.

8. Y. Wen, W. Qu, C. Cheng, Z. Wang, and A. Asundi, "Phase errors elimination in compact digital holoscope (CDH) based on a reasonable mathematical model," (2015), pp. 930207-930207-930209.

9. Y. Wen, W. Qu, Z. Wang, F. Yang, and C. Y. Cheng, "Shape measurement of micro-objects using a common-path digital holographic microscopy (CDHM) with dual wavelength," (2015), pp. 952422-952422-952425.

10. F. Yang, Z. Wang, Y. Wen, and W. Qu, "Two-dimensional phase unwrapping algorithms for fringe pattern analysis: a comparison study," (2015), pp. 93023F93023F-93026.

11. F. Yang, Z. Wang, Y. Wen, and W. Qu, "A quantitative phase imaging system based on transport-of-intensity equation," (2015), pp. 952424-952424-952426. 


\section{REFERENCES}

1. B. Braunecker, R. Hentschel, and H. J. Tiziani, Advanced optics using aspherical elements [electronic resource] / Bernhard Braunecker, Rüdiger Hentschel, Hans J. Tiziani, editors (Bellingham, Wash. : SPIE Press, c2008., 2008).

2. A. Asundi, Digital holography for MEMS and microsystem metrology. [electronic resource] (Chichester : Wiley, c2011., 2011).

3. M. K. Kim, Digital Holographic Microscopy: Principles, Techniques, and Applications (Springer New York, 2013).

4. T.-C. POON, Digital Holography and Three-Dimensional Display: Principles and Applications (Springer Scince Businese Media. INC, 233 Spring street, New York, 2006).

5. J.-M. Asfour, and A. G. Poleshchuk, "Asphere testing with a Fizeau interferometer based on a combined computer-generated hologram," J. Opt. Soc. Am. A 23, 172-178 (2006).

6. D. Visser, T. G. Gijsbers, and R. A. M. Jorna, "Molds and measurements for replicated aspheric lenses for optical recording," Appl. Opt. 24, 1848-1852 (1985).

7. B. C. Platt, and R. Shack, "History and Principles of Shack-Hartmann Wavefront Sensing," Journal of Refractive Surgery Volume 17, 5 (2001).

8. $\quad$ Spiricon, "Hartmann Wavefront Analyzer Tutorial," 19 (2004).

9. D. Malacara, "Hartmann Test of Aspherical Mirrors," Appl. Opt. 11, 99-101 (1972).

10. "Improving the system stability of a digital Shack-Hartmann wavefront sensor with a special lenslet array," Applied Optics 48, A71 (2009).

11. "Measurement of the three-dimensional microscope point spread function using a Shack-Hartmann wavefront sensor : PSF MEASUREMENT USING A WAVEFRONT SENSOR," Journal of Microscopy 205, 61 (2002).

12. M. Rocktäschel, and H. J. Tiziani, "Limitations of the Shack-Hartmann sensor for testing optical aspherics," Optics \& Laser Technology 34, 631-637 (2002).

13. J. M. Bennett, and J. H. Dancy, "Stylus profiling instrument for measuring statistical properties ofsmooth optical surfaces," Appl. Opt. 20, 1785-1802 (1981).

14. $\quad$ K. A. O'Donnell, "Effects of finite stylus width in surface contact profilometry," Appl. Opt. 32, 4922-4928 (1993). 
15. J. F. Song, and T. V. Vorburger, "Stylus profiling at high resolution and low force," Appl. Opt. 30, 42-50 (1991).

16. H.-G. Rhee, Y.-W. Lee, I.-W. Lee, and T. V. Vorburger, "Roughness Measurement Performance Obtained with Optical Interferometry and Stylus Method," J. Opt. Soc. Korea 10, 48-54 (2006).

17. Z.-1. Chen, Z.-d. Guo, Q. Mi, Z.-q. Yang, and R.-b. Bai, "Research of fitting algorithm for coefficients of rotational symmetry aspheric lens," (2009), pp. 728340$728340-72834$.

18. Y. Namba, J. Yu, J. M. Bennett, and K. Yamashita, "Modeling and Measurements of Atomic Surface Roughness," Appl. Opt. 39, 2705-2718 (2000).

19. http://www.zygo.com/?/met/interferometers/verifire/asphere/.

20. E. Garbusi, C. Pruss, and W. Osten, "Interferometer for precise and flexible asphere testing," Opt. Lett. 33, 2973-2975 (2008).

21. G. Baer, J. Schindler, C. Pruss, and W. Osten, "Measurement of Aspheres and Free-Form Surfaces with the Tilted-Wave-Interferometer," in Fringe 2013, W. Osten, ed. (Springer Berlin Heidelberg, 2014), pp. 87-95.

22. Y. Tang, X. Su, Y. Liu, and H. Jing, "3D shape measurement of the aspheric mirror by advanced phase measuring deflectometry," Opt. Express 16, 15090-15096 (2008).

23. Y. Tang, X. Su, F. Wu, and Y. Liu, "A novel phase measuring deflectometry for aspheric mirror test," Opt. Express 17, 19778-19784 (2009).

24. D. Gabor, "A NEW MICROSCOPIC PRINCIPLE," Nature 161, 777-778 (1948).

25. D. Gabor, "Microscopy by Reconstructed Wave-Fronts," Proceedings of the Royal Society of London. Series A, Mathematical and Physical Sciences 197, 454-487 (1949).

26. G. L. Rogers, "Experiments in diffraction microscopy," Proc. Roy. Soc. Edinburgh 63A, 193-221 (1952).

27. a. P. K. H. M. A. El-Sum, "Microscopy by reconstructed wavefronts," Phys. Rev. Lett. 85, 763 (1952).

28. a. T. M. M. E. Haine, "Diffraction Microscopy with X-Rays," Nature 170, 202203 (1952).

29. E. N. Leith, and J. Upatnieks, "Reconstructed Wavefronts and Communication Theory," Journal of the Optical Society of America A: 52, 1123 (1962). 
30. a. J. U. E. N. Leith, "Wavefront reconstruction with continuous-tone objects," J. Opt. Soc. Am. 53, 1377-1381 (1963).

31. a. J. U. E. N. Leith, "Wavefront reconstruction with diffused illumination and three dimensional objects," J. Opt. Soc. Am. 54, 1295-1301 (1964).

32. J. W. Goodman, and R. W. Lawrence, "Digital image formation from electronically detected holograms," Appl. Phys. Lett. 11, 77-79 (1967).

33. U. Schnars, and W. Jüptner, "Direct recording of holograms by a CCD-target and numerical reconstruction," Appl. Opt. 33 179-181 (1994).

34. U. Schnars, "Direct phase determination in hologram interferometry with use of digitally recorded holograms," J. Opt. Soc. Am. A 11, 2011-2015 (1994).

35. U. Schnars, H. J. Hartmann, and W. Jüptner, "Digital recording and numerical reconstruction of holograms for nondestructive testing " Proc. SPIE 2545, 250-253 (1995).

36. E. Cuche, F. Bevilacqua, and C. Depeursinge, "Digital holography for quantitative phase-contrast imaging," Opt. Lett. 24, 291 (1999).

37. E. Cuche, P. Marquet, and C. Depeursinge, "Simultaneous amplitude-contrast and quantitative phase-contrast microscopy by numerical reconstruction of Fresnel off-axis holograms," Appl. Opt. 38, 6994-7001 (1999).

38. a. U. S. W. Jueptner, "Digital Holography: Digital Hologram Recording, Numerical Reconstruction, and Related Techniques," (Springer-Verlag, 2005).

39. U. Schnars, and W. Jüptner, "Digital recording and numerical reconstruction of holograms," Meas. Sci. Technol. 13, R85-R101 (2002).

40. A. Stadelmaier, and J. H. Massig, "Compensation of lens aberrations in digital holography," Opt. Lett. Vol. 25, 3 (2000).

41. G. Pedrini, S. Schedin, and H. J. Tiziani, "Aberration compensation in digital holographic reconstruction of microscopic objects," Journal of Modern Optics 48, 10351041 (2001).

42. P. Ferraro, S. De Nicola, A. Finizio, G. Coppola, S. Grilli, C. Magro, and G. Pierattini, "Compensation of the inherent wave front curvature in digital holographic coherent microscopy for quantitative phase-contrast imaging," Appl. Opt. 42, 1938-1946 (2003).

43. T. Colomb, E. Cuche, F. Charriere, J. Kuhn, N. Aspert, F. Montfort, P. Marquet, and C. Depeursinge, "Automatic procedure for aberration compensation in digital holographic microscopy and applications to specimen shape compensation," Appl. Opt. 45, 851-863 (2006). 
44. T. Colomb, J. Kühn, F. Charrière, C.Depeursinge, P. Marquet, and N. Aspert, "Total aberrations compensation in digital holographic microscopy with a reference conjugated hologram," Opt. Express 14, 4300 (2006).

45. T. Colomb, F. Montfort, J. Kühn, N. Aspert, E. Cuche, A. Marian, F. Charrière, S. Bourquin, P. Marquet, and C. Depeursinge, "Numerical parametric lens for shifting, magnification, and complete aberration compensation in digital holographic microscopy," J. Opt. Soc. Am. A 23, 3177 (2006).

46. F. Montfort, F. Charrière, T. Colomb, E. Cuche, P. Marquet, and a. C. Depeursinge, "Purely numerical compensation for microscope objective phase curvature in digital holographic microscopy: influence of digital phase mask position," J. Opt. Soc. Am. A 23, 2944 (2006).

47. L. Miccio, D. Alfieri, S. Grilli, P. Ferraro, A. Finizio, L. De Petrocellis, and S. D. Nicola, "Direct full compensation of the aberrations in quantitative phase microscopy of thin objects by a single digital hologram," Applied Physics Letters 90 (2007).

48. W. J. Qu, Y. J. Yu, C. O. Choo, and A. Asundi, "Digital holographic microscopy with physical phase compensation," Opt. Lett. 34, 1276-1278 (2009).

49. C. Wagner, W. Osten, and S. Seebacher, "Direct shape measurement by digital wavefront reconstruction and multiwavelength contouring " Opt. Eng. 39, 79-85 (2000).

50. J. Gass, A. Dakoff, and M. K. Kim, "Phase imaging without 2 pi ambiguity by multiwavelength digital holography," Opt. Lett. 28, 1141-1143 (2003).

51. B. Javidi, P. Ferraro, S.-H. Hong, S. D. Nicola, A. Finizio, D. Alfieri, and G. Pierattini, "Three-dimensional image fusion by use of multiwavelength digital holography," Opt. Lett. Vol. 30, 3 (2005).

52. S. D. Nicola, A. Finizio, G. Pierattini, D. Alfieri, S. Grilli, L. Sansone, and P. Ferraro, "Recovering correct phase information in multiwavelength digital holographic microscopy by compensation for chromatic aberrations," Opt. Lett. Vol. 30, 3 (2005).

53. H. Kadono, M. Ogusu, and S. Toyooka, "PHASE-SHIFTING COMMON-PATH INTERFEROMETER USING A LIQUID-CRYSTAL PHASE MODULATOR," Optics Communications 110, 391-400 (1994).

54. I. Yamaguch, and T. Zhang, "Phase-shifting digital holography," Opt. Lett. 22, 1268-1270 (1997).

55. T. Zhang, and I. Yamaguchi, "Three-dimensional microscopy with phase-shifting digital holography," Opt. Lett. 23, 1221-1223 (1998).

56. S. C. Lai, B. King, and M. A. Neifeld, "Wave front reconstruction by means of phase-shifting digital in-line holography," Optics Communications 173, 155-160 (2000). 
57. I. Yamaguchi, J.Kato, S. Ohta, and J. Mizuno, "Image Formation in PhaseShifting Digital Holography and Applications to Microscopy," Appl. Opt. 40, 6177-6186 (2001).

58. I. Yamaguchi, S. Ohta, and J. Kato, "Surface shape measurement by phaseshifting digital holography," Opt. Rev. 8, 85-89 (2001).

59. C.-S. Guo, L. Zhang, H.-T. Wang, J. Liao, and Y. Y. Zhu, "Phase-shifting error and its elimination in phase-shifting digital holography," Opt. Lett. Vol. 27, 3 (2002).

60. I. Yamaguchi, T. Matsumura, and J.-I. Kato, "Phase-shifting color digital holography," Opt. Lett. Vol. 27, 3 (2002).

61. H. Iwai, C. Fang-Yen, G. Popescu, P. Wax, K. Badizadegan, R. R. Dasari, and M. S. Feld, "Quantitative phase imaging using actively stabilized phase-shifting lowcoherence interferometry," Opt. Lett. Vol. 29, 3 (2004).

62. I. Yamaguchi, K. Yamamoto, G. A. Mills, and M. Yokota, "Image reconstruction only by phase data in phase-shifting digital holography," Appl. Opt. Vol. 45, 9 (2006).

63. V. Mico, Z. Zalevsky, and J. Garcia, "Common-path phase-shifting digital holographic microscopy: A way to quantitative phase imaging and superresolution," Optics Communications 281, 4273-4281 (2008).

64. C.Remmersmann, S. Stürwald, B. Kemper, P. Langehanenberg, and a. G. v. Bally, "Phase noise optimization in temporal phase-shifting digital holography with partial coherence light sources and its application in quantitative cell imaging," Appl. Opt. 48, 1463-1472 (2009).

65. A. Asundi, "Digital Holography for MEMS characterization and Micro-particles tracking," 33.

66. A. Asundi, "Dynamic Characterization and Reliability of MEMS," 6.

67. V. Kebbel, J. Müller, and W. P. O. Jüptner, "Characterization of aspherical microoptics using digital holography: improvement of accuracy," 10.

68. V. Kebbel, H.-J. Hartmann, and W. P. O. Jüptner, "Characterization of microoptics using digital holography," Proceedings of SPIE Vol. 4101, 11 (2000).

69. C. Furlong, J. S. Yokum, C. A. Phillips, and R. J. Pryputniewicz, "Optoelectronic holography shape characterization of microspheres for biomedical applications," Proc. Symp. on MEMS: Mechanics and Measurements, 4 (2002).

70. G. Coppola, P. Ferraro, M. Iodice, S. D. Nicola, A. Finizio, and S. Grilli, "A digital holographic microscope for complete characterization of microelectromechanical systems," MEASUREMENT SCIENCE AND TECHNOLOGY 15, 11 (2004). 
71. L. Tec, "Dynamic characterization of MEMS and MOEMS," 2 (2006).

72. $\quad$ L. Tec, "Characterization of micro optics with Digital Holographic Microscopy," 2 (2006).

73. W. J. Qu, C. O. Choo, Y. J. Yu, and A. Asundi, "Microlens characterization by digital holographic microscopy with physical spherical phase compensation," Appl. Opt. 49, 6448-6454 (2010).

74. M. K. Kim, "Tomographic three-dimensional imaging of a biological specimen using wavelength-scanning digital interference holography," Opt. Express Vol. 7, 6 (2000).

75. X. Wenbo, M. H. Jericho, I. A. Meinertzhagen, and H. J. Kreuzer, "Digital in-line holography for biological applications," Proceedings of the National Academy of Sciences of the United States 98, 11301 (2001).

76. P. J. McMahon, E. D. Barone-Nugent, B. E. Allman, and K. A. Nugent, "Quantitative phase-amplitude microscopy II: differential interference contrast imaging for biological TEM," Journal of Microscopy-Oxford 206, 204-208 (2002).

77. S. A. Alexandrov, P. Meredith, T. J. McIntyre, and A. V. Zvyagin, "Holographic Digital Fourier Microscopy for Selective Imaging of Biological Tissue," Inc. Int J Imaging Syst Technol 14, 6 (2004).

78. M. d. S. Hernández-Montes, C. Pérez-López, F. M. Santoyo, and L. M. M. Guevara, "Detection of biological tissue in gels using pulsed digital holography," Opt. Express Vol. 12, 6 (2004).

79. S. Tanev, W. B. Sun, R. Zhang, and A. Ridsdale, "Simulation tools solve lightscattering problems from biological cells," Laser Focus World 40, 67-70 (2004).

80. C. D. Depeursinge, A. M. Marian, F. Montfort, T. Colomb, F. Charriere, J. Kuhn, N. Aspert, M. Botkine, M. S. Ghislain, F. Marquet, E. Cuche, Y. Emery, P. Marquet, and P. J. Magistretti, "Digital Holographic Microscopy, a new imaging technology applied to biological cells and tissues.," Proceedings of SPIE, 1 (2005).

81. I. Moon, and B. Javidi, "Shape tolerant three-dimensional recognition of biological microorganisms using digital holography," Opt. Express Vol. 13, 11 (2005).

82. O. Veselov, J. Lekki, W. Polak, D. Strivay, Z. Stachura, K. Lebed, and J. Styczen, "The recognition of biological cells utilizing quantitative phase microscopy system," Nuclear Instruments \& Methods in Physics Research Section B-Beam Interactions with Materials and Atoms 231, 212-217 (2005).

83. T. Colomb, J. Kühn, F. Charrière, C. Depeursinge, P. Marquet, and N. Aspert, "Real-time Phase Recovery of Biological Cell in Digital Holographic Microscopy by Use of a Self-Calibration Hologram," 3 (2006). 
84. B. Javidi, I. Moon, and S. Yeom, "Real time automated 3D imaging and monitoring of dynamic microscopic biological events," 8 (2006).

85. H. J. K. a. H. Jericho, "Holographicmicroscopy gives new tool for biology and microfluidics," SPIE 6112 (2006).

86. L. Tec, "Biological specimen 3D morphology and refractive index separation," 2 (2006).

87. L. Tec, "Real-time 3D monitoring of living biological specimens," 2 (2006).

88. M. C. Potcoava, and M. K. Kim, "Optical tomography for biomedical applications by digital interference holography," MEASUREMENT SCIENCE AND TECHNOLOGY 19, 8 (2008).

89. W. Jueptner, Digital holography (Springer, 2001).

90. J. W. GOODMAN, Introduction to Fourier Optics (McGRAW-HILL, 1996).

91. M. Blum, M. Büeler, C. Grätzel, and M. Aschwanden, "Compact optical design solutions using focus tunable lenses," (2011), pp. 81670W-81670W-81679.

92. C. Zuo, Q. Chen, and A. Asundi, "Phase retrieval and computational imaging with the transport of intensity equation: boundary conditions, fast solution, and discrepancy compensation," in Classical Optics 2014(Optical Society of America, Kohala Coast, Hawaii, 2014), p. CTh3C.3.

93. G. Eberle, V. Chiron, and K. Wegener, "Simulation and Realization of a Focus Shifting Unit using a Tunable Lens for 3D Laser Material Processing," Physics Procedia 41, 441-447 (2013).

94. M. Blum, M. Büeler, C. Grätzel, J. Giger, and M. Aschwanden, "Optotune focus tunable lenses and laser speckle reduction based on electroactive polymers," (2012), pp. 825207-825207-825211.

95. C. Graetzel, M. Suter, and M. Aschwanden, "Reducing laser speckle with electroactive polymer actuators," (2015), pp. 943004-943004-943008.

96. Y. K. Tao, S. K. Srivastava, and J. P. Ehlers, "Microscope-integrated intraoperative OCT with electrically tunable focus and heads-up display for imaging of ophthalmic surgical maneuvers," Biomed. Opt. Express 5, 1877-1885 (2014).

97. B. F. Grewe, F. F. Voigt, M. van 't Hoff, and F. Helmchen, "Fast two-layer twophoton imaging of neuronal cell populations using an electrically tunable lens," Biomed. Opt. Express 2, 2035-2046 (2011).

98. K. Lee, E. Chung, and T. J. Eom, "Multi-depth photoacoustic microscopy with a focus tunable lens," (2015), pp. 93233O-93233O-93236. 
99. C. Zuo, Q. Chen, W. Qu, and A. Asundi, "High-speed transport-of-intensity phase microscopy with an electrically tunable lens," Opt. Express 21, 24060-24075 (2013).

100. F. O. Fahrbach, F. F. Voigt, B. Schmid, F. Helmchen, and J. Huisken, "Rapid 3D light-sheet microscopy with a tunable lens," Opt. Express 21, 21010-21026 (2013).

101. J. A. Rodrigo, and T. Alieva, "Rapid quantitative phase imaging for partially coherent light microscopy," Opt. Express 22, 13472-13483 (2014).

102. D. Malacara, and Z. Malacara, Handbook of optical design. [electronic resource] (Boca Raton, FL : Taylor \& Francis, 2013.

3rd ed., 2013).

103. P. Török, and F.-J. Kao, Optical Imaging and Microscopy. [electronic resource] : Techniques and Advanced Systems (Berlin, Heidelberg : Springer-Verlag, 2007.

2., 2007).

104. P. Hariharan, Optical interferometry. [electronic resource] (Amsterdam ; Boston : Academic Press, c2003.

2nd ed., 2003).

105. T. Kreis, Handbook of Holographic Interferometry (2005).

106. D. Malacara, Optical shop testing. [electronic resource] (Hoboken, N.J. : WileyInterscience, c2007.

3rd ed., 2007).

107. S. Grare, D. Allano, S. Coëtmellec, G. Perret, F. Corbin, M. Brunel, G. Gréhan, and D. Lebrun, "Dual-wavelength digital holography for 3D particle image velocimetry: experimental validation," Appl. Opt. 55, A49-A53 (2016).

108. D. Boss, J. Kuehn, C. Depeursinge, P. J. Magistretti, P. E. D. B. E. Marquet, and P. So, "Dual-wavelength Digital Holography for quantification of cell volume and integral refractive index (RI)," in Advanced Microscopy Techniques II(Optical Society of America, Munich, 2011), p. 808608.

109. H.-J. Cho, D.-C. Kim, Y.-H. Yu, W.-K. Jung, and S.-H. Shin, "Dual-wavelength Digital Holography Microscope for BGA Measurement Using Partial Coherence Sources," J. Opt. Soc. Korea 15, 352-356 (2011).

110. B. P. Thiesing, C. J. Mann, and S. Dryepondt, "High temperature measurements of martensitic transformations using digital holography," Appl. Opt. 52, 4426-4432 (2013). 
111. T. Hansel, G. Steinmeyer, R. Grunwald, U. Griebner, F. Schneider, and U. Wallrabe, "High-Speed Contouring of an Adaptive Fluidic Lens with Two-Wavelength Single-Pulse Digital Holography," in Advances in Imaging(Optical Society of America, Vancouver, 2009), p. DMA6.

112. B. Rappaz, F. Charrière, C. Depeursinge, P. J. Magistretti, and P. Marquet, "Simultaneous cell morphometry and refractive index measurement with dualwavelength digital holographic microscopy and dye enhanced dispersion of perfusion medium " Opt. Lett. 33, 744-746 (2008).

113. A. Khmaladze, A. Restrepo-Martínez, M. Kim, R. Castañeda, and A. Blandón, "Simultaneous dual-wavelength reflection digital holography applied to the study of the porous coal samples," Appl. Opt. 47, 3203-3210 (2008).

114. J. Kühn, T. Colomb, F. Montfort, F. Charrière, Y. Emery, E. Cuche, P. Marquet, and C. Depeursinge, "Real-time dual-wavelength digital holographic microscopy with a single hologram acquisition," Opt. Express 15, 7231-7242 (2007).

115. T. Tahara, and Y. Arai, "Space-bandwidth extension in single-shot off-axis digital holography using dual-wavelength phase unwrapping," in JSAP-OSA Joint Symposia 2013 Abstracts(Optical Society of America, Kyoto, 2013), p. 17p_D15_17.

116. Z. Wang, Z. Jiang, Y. Chen, and Y. Zhao, "Single-shot dual-wavelength digital holography with polarization-multiplexing transmission," in Digital Holography \& 3-D Imaging Meeting(Optical Society of America, Shanghai, 2015), p. DM2A.7.

117. D. G. Abdelsalam, R. Magnusson, and D. Kim, "Single-shot, dual-wavelength digital holography based on polarizing separation," Appl. Opt. 50, 3360-3368 (2011).

118. Z. Wang, Z. Jiang, and Y. Chen, "Single-shot dual-wavelength phase reconstruction in off-axis digital holography with polarization-multiplexing transmission," Appl. Opt. 55, 6072-6078 (2016).

119. J. C. Wyant, "Extended Range Two-Wavelength Interferometry," Optical Testing.

120. D. Parshall, and M. K. Kim, "Digital holographic microscopy with dualwavelength phase unwrapping," Appl. Opt. 45, 451-459 (2006).

121. A. Khmaladze, R. L. Matz, C. Zhang, T. Wang, M. M. Banaszak Holl, and Z. Chen, "Dual-wavelength linear regression phase unwrapping in three-dimensional microscopic images of cancer cells," Opt. Lett. 36, 912-914 (2011).

122. R. C. Gonzalez, and R. E. Woods, Digital Image Processing (Pearson/Prentice Hall, 2008).

123. A. Asundi, V. R. Singh, and P. Zhong, "Digital Holography - A new paradigm In Imaging, Microscopy and Metrology," 21 (2006). 
124. D. C. Ghiglia, and M. D. Pritt, Two-Dimensional Phase Unwrapping: Theory, Algorithms, and Software (Wiley, 1998).

125. F. Le Clerc, M. Gross, and L. Collot, "Synthetic-aperture experiment in the visible with on-axis digital heterodyne holography," Opt. Lett. 26, 1550-1552 (2001).

126. J. H. Massig, "Digital off-axis holography with a synthetic aperture," Opt. Lett. 27, 2179-2181 (2002).

127. R. Binet, J. Colineau, and J.-C. Lehureau, "Short-range synthetic aperture imaging at $633 \mathrm{~nm}$ by digital holography," Appl. Opt. 41, 4775-4782 (2002).

128. L. Martínez-León, and B. Javidi, "Synthetic aperture single-exposure on-axis digital holography," Opt. Express 16, 161-169 (2008).

129. G. Indebetouw, A. El Maghnouji, and R. Foster, "Scanning holographic microscopy with transverse resolution exceeding the Rayleigh limit and extended depth of focus," Journal of the Optical Society of America. A, Optics, image science, and vision 22, 892-898 (2005).

130. C. Yuan, H. Zhai, and H. Liu, "Angular multiplexing in pulsed digital holography for aperture synthesis," Opt. Lett. 33, 2356-2358 (2008).

131. J. Di, J. Zhao, H. Jiang, P. Zhang, Q. Fan, and W. Sun, "High resolution digital holographic microscopy with a wide field of view based on a synthetic aperture technique and use of linear CCD scanning," Appl. Opt. 47, 5654-5659 (2008).

132. M. Paturzo, and P. Ferraro, "Correct self-assembling of spatial frequencies in super-resolution synthetic aperture digital holography," Opt. Lett. 34, 3650-3652 (2009).

133. H. Jiang, J. Zhao, J. Di, and C. Qin, "Numerically correcting the joint misplacement of the sub-holograms in spatial synthetic aperture digital Fresnel holography," Opt. Express 17, 18836-18842 (2009).

134. P. Feng, X. Wen, and R. Lu, "Long-working-distance synthetic aperture Fresnel off-axis digital holography," Opt. Express 17, 5473-5480 (2009).

135. D. Claus, "High resolution digital holographic synthetic aperture applied to deformation measurement and extended depth of field method," Appl. Opt. 49, 31873198 (2010).

136. L. Granero, V. Micó, Z. Zalevsky, and J. García, "Synthetic aperture superresolved microscopy in digital lensless Fourier holography by time and angular multiplexing of the object information," Appl. Opt. 49, 845-857 (2010).

137. M. Kim, Y. Choi, C. Fang-Yen, Y. Sung, R. R. Dasari, M. S. Feld, and W. Choi, "High-speed synthetic aperture microscopy for live cell imaging," Opt. Lett. 36, 148-150 (2011). 
138. Y. Hao, and A. Asundi, "Studies on aperture synthesis in digital Fresnel holography," Optics and Lasers in Engineering 50, 556-562 (2012).

139. A. E. Tippie, A. Kumar, and J. R. Fienup, "High-resolution synthetic-aperture digital holography with digital phase and pupil correction," Opt. Express 19, 1202712038 (2011).

140. https://www.microsoft.com/en-us/research/product/computational-photographyapplications/image-composite-editor/.

141. D. Malacara, and Z. Malacara, Handbook of optical design (Marcel Dekker, Inc., 2004).

142. R. E. Fischer, B. Tadic-Galeb, and P. R. Yoder, Optical system design. [electronic resource] (New York : McGraw-Hill, c2008.

2nd ed., 2008).

143. Z. Zhang, "Parameter estimation techniques: a tutorial with application to conic fitting," Image and Vision Computing 15, 59-76 (1997).

144. M. Jung, K. J. Cross, J. W. McBride, and M. Hill, "A method for the selection of algorithms for form characterization of nominally spherical surfaces," Precision Engineering 24, 127-138 (2000).

145. F. Xie, Q. Hao, Q. Zhu, L. Tang, and Y. Hu, "A best-fit sphere definition capable of reducing dynamic range in aspheric surface testing," (2010), pp. 784917-784917784917.

146. G. W. Forbes, "Shape specification for axially symmetric optical surfaces," Opt. Express 15, 5218-5226 (2007).

147. J. Guo, Y. Guo, J. Wang, and T. Zhang, "Measuring path planning and surface fitting technology for non-axisymmetric aspheric lens," (2008), pp. 71300I-71300I-71307.

148. S. Arba-Mosquera, J. Merayo-Lloves, and D. de Ortueta, "Asphericity analysis using corneal wavefront and topographic meridional fits," BIOMEDO 15, 028003028003-028009 (2010).

149. D.-I. Kim, G. Kim, and G. H. Kim, "Effective method for extracting aspheric parameters inherent in unknown aspheric surfaces," (2015), pp. 962815-962815-962818.

150. X. Cheng, Y. Yang, and Q. Hao, "Aspherical surface profile fitting based on the relationship between polynomial and inner products," OPTICE 55, 015105-015105 (2016). 
151. N. El-Hayek, H. Nouira, N. Anwer, O. Gibaru, and M. Damak, "A new method for aspherical surface fitting with large-volume datasets," Precision Engineering 38, 935947 (2014).

152. W. Sun, J. W. McBride, and M. Hill, "A new approach to characterising aspheric surfaces," Precision Engineering 34, 171-179 (2010).

153. G. Forbes, "Better Ways to Specify Aspheric Shapes Can Facilitate Design, Fabrication and Testing Alike," in International Optical Design Conference and Optical Fabrication and Testing(Optical Society of America, Jackson Hole, Wyoming, 2010), p. JMA1.

154. G. W. Forbes, "Robust and fast computation for the polynomials of optics," Opt. Express 18, 13851-13862 (2010).

155. G. W. Forbes, "Robust, efficient computational methods for axially symmetric optical aspheres," Opt. Express 18, 19700-19712 (2010).

156. G. W. Forbes, "Characterizing the shape of freeform optics," Opt. Express 20, 2483-2499 (2012).

157. G. W. Forbes, "Fitting freeform shapes with orthogonal bases," Opt. Express 21, 19061-19081 (2013).

158. W. Ford, "Chapter 14 - Gram-Schmidt Orthonormalization," in Numerical Linear Algebra with Applications(Academic Press, 2015), pp. 281-297. 